\title{
From Hadrons to Nuclei: Crossing the Border
}

\author{
Silas R. Beane ${ }^{a}$, Paulo F. Bedaque ${ }^{b}$, Wick C. Haxton ${ }^{b}$ \\ Daniel R. Phillips ${ }^{a, c}$, and Martin J. Savage ${ }^{a, d}$ \\ a Department of Physics, \\ University of Washington, Seattle, WA 98195-1560 \\ ${ }^{b}$ Institute for Nuclear Theory, \\ University of Washington, Seattle, WA 98195-1560 \\ ${ }^{c}$ Department of Physics, \\ Ohio University, Athens, OH 45701 \\ d Jefferson Laboratory, \\ 12000 Jefferson Avenue, Newport News, VA 23606 \\ sbeane, bedaque, phillips, savage, haxton@phys.washington.edu
}

The study of nuclei predates by many years the theory of quantum chromodynamics. More recently, effective field theories have been used in nuclear physics to "cross the border" from QCD to a nuclear theory. We are now entering the second decade of efforts to develop a perturbative theory of nuclear interactions using effective field theory. This chapter describes the current status of these efforts.

\section{Introduction}

Nuclei are fascinating objects. In a nucleus the protons and neutrons, collectively known as nucleons, are bound together by the strong nuclear force. At a fundamental level these interactions are described by Quantum Chromodynamics (QCD), a theory of quarks and gluons carrying color charges that are asymptotically-free at short-distances. However, the quarks and gluons in a nucleus are very far from being asymptotically-free. Instead they comprise individual, colorless, nucleons, which largely retain their identity in the many-body system. The color-singlet nucleons are then bound to each other by what can be thought of as "residual" QCD strong interactions. This sketch of nuclear dynamics from the QCD point of view-brief as it is - makes it clear that from this standpoint the nucleus is an incredibly complicated, nonperturbative, quantum-field-theoretic, infinite-body problem.

This makes it truly remarkable that the properties and interactions of nuclei can be largely understood within the following framework, which we shall refer to here as the "traditional" picture of a nucleus. The $A$ nucleons in a nucleus are understood to be non-relativistic particles interacting via a 
quantum-mechanical Hamiltonian consisting of two-body potentials and threebody potentials which have considerably smaller, but still important, effects:

$$
H=\sum_{i=1}^{N} \frac{\left|\mathbf{p}_{\mathbf{i}}\right|^{2}}{2 M}+\sum_{i<j} V_{N N}\left(\mathbf{r}_{i}, \mathbf{r}_{j}\right)+\sum_{i<j<k} V_{3 N}\left(\mathbf{r}_{i}, \mathbf{r}_{j}, \mathbf{r}_{k}\right) .
$$

This Hamiltonian is then used in the Schrödinger equation

$$
H\left|\psi^{(i)}\right\rangle=E^{(i)}\left|\psi^{(i)}\right\rangle,
$$

to determine the nuclear wave functions $\left|\psi^{(i)}\right\rangle$ and energy-levels $E^{(i)}$. With modern computing power the energy-levels of this Hamiltonian can be calculated with $\sim 1 \%$ precision for nuclei as large as $A=8$ and soon calculations for twelve-body nuclei such as ${ }^{12} C$ should become available. The results of this program are in impressive agreement with experiment. With wave functions in hand, properties of the nuclides such as magnetic and quadrupole moments, electromagnetic form factors, and the response of light nuclei to weak probes such as neutrinos can all be calculated. For a thorough review of these successes see Ref.t.

However, the success of a picture described by the Hamiltonian in Eq. (11) raises a number of other questions, among which are:

- Why are nucleons the appropriate degrees of freedom inside a nucleus?

- Why are two-body interactions $V_{N N}$ dominant? And how should they be described?

- How do we construct a three-nucleon interaction $V_{3 N}$ ?

- Are all contributions to observables equally large, or is there some set of small parameters which can be used to organize the calculation?

All of these issues are part of one overarching question, namely:

Can we both understand how the Hamiltonian in Eq. (1) emerges from QCD to dominate the dynamics of multi-nucleon systems, and systematically refine it to accurately describe all nuclear observables.

One kind of answer to this question would be provided by computations of the properties and reactions of nuclei directly from lattice QCD. For instance, consider what happens when a proton captures a neutron and forms a deuteron, in the process emitting a photon: the radiative capture process 
$n p \rightarrow d \gamma$. A lattice calculation of $n p \rightarrow d \gamma$ would provide an unambiguous cross-section in terms of the quark masses and $\Lambda_{\mathrm{QCD}}$. However, such a calculation would be very difficult to perform. For instance, a cartoon of one contribution to $n p \rightarrow d \gamma$ in terms of perturbative quarks and gluons is shown in Fig. 1. Sources of quarks and gluons that have non-zero overlap with the proton, neutron, and deuteron as well as a source for the photon would be used to generate the reaction amplitude. One key problem with using lattice QCD to calculate this reaction is that a significant amount of effort goes into forming the asymptotic states themselves - before one even begins computing their interactions. Indeed, since the deuteron has such a small binding energy and so is quite extended compared to the nucleon, considerable effort will be required to generate the deuteron on the lattice. The deuteron is yet to be formed in any lattice calculation, and so, to put it mildly, a comprehensive lattice study of this simple hadronic process is not imminent. Therefore, given

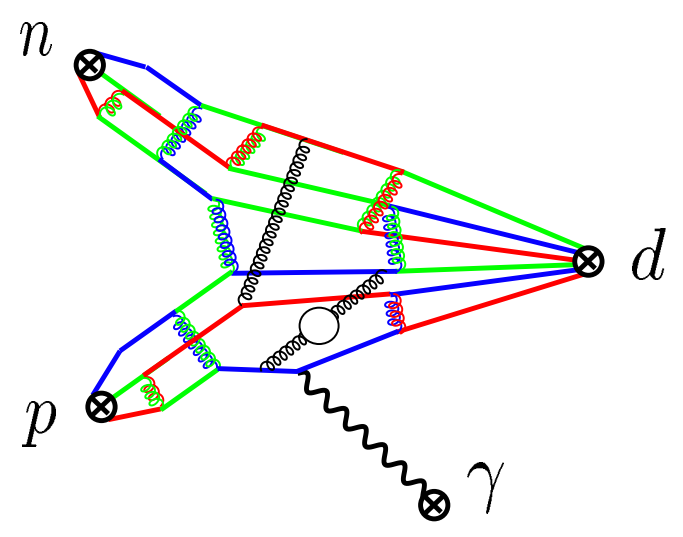

Figure 1: A "cartoon" of a contribution to $n p \rightarrow d \gamma$ in QCD.

the current state of affairs in lattice QCD, serious efforts to determine properties of multi-nucleon systems must start with neutrons and protons as the basic degrees of freedom. This has the considerable advantage that one is working in terms of the asymptotic states which are those detected in the laboratory.

Clearly an important first step in describing the dynamics of a multinucleon system is to describe the interaction between just two-nucleons, i.e. the two-nucleon scattering amplitude. This has been accomplished very successfully by fitting the nucleon-nucleon $(N N)$ potential $V_{N N}$. There are many $N N$ potentials on the market, but the most accurate models are now tuned to fit the over 3000 data which constitute the database for $N N$ scattering below labora- 
tory energies of $350 \mathrm{MeV}$. In all of these models the long-distance part of the interaction, corresponding to inter-nucleon distances of order $2 \mathrm{fm} \sim 1 / m_{\pi}$, is described by the model-independent exchange of one pion - a sixty-five year old idea, due to Yukawa 3 . At shorter distances $\sim 1 /\left(2 m_{\pi}\right)$ some potentials include the exchange of two pions. This too can be derived in a model-independent way from chiral perturbation theory $(\chi \mathrm{PT})$. In contrast, once one looks at the force between nucleons separated by $\sim 1 \mathrm{fm}$, there are a variety of short-range components present in the different $N N$ potentials currently in use. Some assert that the short-range interaction arises from the exchange of vector and scalar mesons, while others look to the naive quark model for inspiration. More popular, and more effective, than either of these models are potentials which fit the data essentially to within experimental accuracy 1.6 , using a short-distance part of $V_{N N}$ that is essentially a best fit, with no dynamical assumptions other than continuity, differentiability, and the desire to include all possible operator structures which can arise in the $N N$ system at low energies. This part of $V_{N N}$ occurs in the region where the nucleons themselves have substantial overlap, and $r \sim \Lambda_{\chi}^{-1}$ with $\Lambda_{\chi}$ is the chiral-symmetry breaking scale. It is clear that without input from lattice QCD, assertions about the underlying dynamics in this region are nothing more than guess-work. Thus the use of these "best-fit" short-distance $N N$ interactions is very much in the spirit of effective field theory. However, once we move beyond the $N N$ interaction and start considering three-nucleon forces and the construction of current operators for use in multi-nucleon systems the picture becomes significantly more complex. As nuclear systems are inherently non-perturbative it is difficult to see which parts of a computation are essential to the description of a particular observable, and which parts are not. Effective field theory (EFT) allows one to both systematize calculations in light nuclei, and simplify the picture of these nuclei down to its essential ingredients.

In this chapter of the Handbook of $Q C D$ we will describe the progress that has been made in constructing and applying EFT's to multi-nucleon systems, and the interactions of these systems with "external" probes such as $\gamma^{\prime}$ 's, $W^{ \pm}$'s, $Z^{0}$ 's, and $\pi$. Since Weinberg's pioneering efforts 6 , in the early 1990 s to set up a systematic description of multi-nucleon interactions using EFT much effort has been gone into realizing this goal. At present these nuclear EFTs are far from being able to determine, for instance, the $B(E 2)$ values for transitions

\footnotetext{
${ }^{a}$ In Ref. 2 this was chosen as the uppermost energy for which data would be included in the $N N$ database because the pion-production threshold, at $T_{\text {lab }}=280 \mathrm{MeV}$, begins to play a role there, and so the $N N$ collisions are no longer elastic.

${ }^{b}$ There is an old saying: Those who do not know history are doomed to repeat it. However, there is a slightly more modern version that may be more relevant: Those who do not understand history will not recognize progress.
} 
in ${ }^{25} \mathrm{Mg}$, but important progress has been made in understanding systems of two and three nucleons as well as in discussion of probes of the two-nucleons system. We will also discuss the progress and problems facing EFT's attempts to discuss larger nuclei.

An EFT is defined by the most general Lagrange density consistent with the global and local symmetries of the underlying theory, a declaration of the regularization and renormalization scheme, and a well-defined power counting in terms of small expansion parameters. This issue is somewhat controversial in the presence of bound- or quasi-bound states because there are differing criteria for what constitutes a rigorous EFT. On the one hand there is cutoff EFT. In cutoff EFT the power counting is essentially dimensional analysis at the level of the potential: operators are ordered according to inverse powers of the momentum space cutoff. Since the quantum mechanical potential is not an observable quantity, the justification for the power counting is necessarily a posteriori. One computes to a given order in the expansion and then one performs an error analysis for observables such as phase shifts in order to verify that cutoff dependence is removed order-by-order in the expansion of the potential. This method has much in common with lattice gauge theory with improved lattice actions 10 . Lattice errors are removed order-by-order in the lattice spacing by including operators in the action with increasing powers of the lattice spacing. On the other hand, there is EFT as it is traditionally practiced in a purely perturbative setting, like chiral perturbation theory $(\chi P T)$. The power counting is in the scattering amplitude itself and therefore applies directly to observable quantities. In this case one can explicitly prove renormalization scale independence at each order in the EFT expansion of observables. Moreover, one can make an a priori estimate of the theoretical errors which arise from working to a given order in the perturbative expansion. The distinction between these two criteria becomes an issue only when there is a bound- or quasi-bound state near threshold. In these cases at least one local (as in a contact operator) or non-local operator (as in a particle exchange) must be summed to all orders and so the EFT is intrinsically nonperturbative. The nonperturbative nature of the $N N$ interaction leads to several unresolved formal issues in a cutoff EFT of $N N$ interactions. These issues will be discussed below.

The underlying motivation for using EFT to describe systems with wellseparated lengths scales can be found in the Chapters by Leutwyler, by Meißner and by Manohar, and we will not repeat it here. Instead, we begin our tour of EFTs in nuclear physics by looking at the simplest nuclear system, $A=2$, at momenta well below the pion mass. At these very low energies the only relevant degrees of freedom are the nucleons themselves. We will show how to 
systematically describe both $N N$ scattering and the coupling of electroweak probes to the $N N$ system. These calculations of very-low-energy properties provide analytic results for all observables. They must be compared, processby-process, against the results of the "traditional" approach. Subsequently, we move away from the $A=2$ system and discuss EFT for the $A=3$ system-also at very low energies. The EFT treatment of the three-nucleon problem provides some truly novel features, including the emergence of a type of renormalizationgroup (RG) behavior not seen before in a single-parameter system.

We then turn to higher energies, where the pion must be included as an explicit degree of freedom in the Lagrangian. Attempts to include pions in perturbation theory are presented and their current status discussed. We then discuss Weinberg's original suggestion for dealing with the pions nonperturbatively. This is to apply chiral perturbation theory $(\chi \mathrm{PT})$, as formulated in the Chapters by Leutwyler and Meißner, to the $N N$ potential and other two-nucleon irreducible operators. We display some successes of this approach, and discuss some unresolved formal issues. Finally, we discuss recent progress in incorporating the ideas of EFT into more traditional methods of calculating nuclear properties.

\section{Two-Nucleon Systems at Very Low Energies}

Ideas akin to EFT appeared in nuclear physics as early as the 1940s, when Bethe introduced Effective Range Theory (ERT) as a description of $N N$ scattering 11.12. Bethe showed that at low energy the experimental data did not depend on dynamical details, but only on a few numbers which summarized the impact of strong interactions on observables. ERT is an attempt to describe both elastic and inelastic processes involving two nucleons, provided that all momenta involved are less than the pion mass. By construction, strong-interaction data is reproduced in ERT to arbitrary precision in this low-momentum regime. However, ERT fails to converge to the measured amplitudes for processes involving photons or other "external" probes. As the energy of experiments increased, ERT become less relevant, and more complicated descriptions in the form of potential models, such as the Hamiltonian (11), became popular. In this section we will show how to derive an EFT which will reproduce ERT, and hence the $N N$ data, to arbitrary precision, provided that $p<m_{\pi} / 2$. EFT goes beyond ERT, as it systematically includes operators which enter when one calculates the response of the $N N$ system to external probes. We will give explicit examples of analytic calculations for low-energy reactions which can be carried out to $\sim 1 \%$ accuracy. 


\subsection{Naive Dimensional Analysis Really is Naive}

What scales might be reasonably expected in the two-nucleon sector if one was simply presented with the QCD Lagrangian? Neglecting quark masses, $\mathrm{QCD}$ is a theory with only one length scale, $\Lambda_{\mathrm{QCD}}$. In the hadronic sector this scale appears as the scale of chiral symmetry breaking, $\Lambda_{\chi} \sim 1 \mathrm{GeV}$, and the nucleon mass $M$. At low energies, the effective theory of QCD will be one whose matter content includes only non-relativistic nucleons and massless pions. Neglecting factors of $4 \pi$ the only mass scale in the theory is $\Lambda_{\chi}$ ?

The inclusion of the quark masses in the QCD Lagrangian explicitly breaks chiral symmetry. The pion acquires a mass $m_{\pi} \approx 140 \mathrm{MeV}$, and so introduces another "small" length scale. What then, is a reasonable scale to expect for the binding energy of nuclei? Naively one might expect that the nuclear size will be set by the pion mass. However, one must remember that the pion is derivatively coupled, and so it is not clear that the pionic dynamics are strong enough to be responsible for bound-state formation. Even accepting that the pion is the particle whose exchange generates the non-perturbative interaction which leads to the formation of the deuteron, one would not then expect it to be bound by only $B=2.224644 \pm 0.000034 \mathrm{MeV}$. This corresponds to a pole in the $N N$ scattering amplitude at momentum $|\mathbf{k}|=i \gamma$-with $\gamma \equiv$ $\sqrt{M B}=45.7025 \mathrm{MeV}$-in the ${ }^{3} S_{1}$ channel fl: significantly less than our prior expectation. In the ${ }^{1} S_{0}$ channel the situation is even more striking. There is no bound-state in the ${ }^{1} S_{0}$ channel, but a pole on the second energy-sheet represents a quasi-bound state. In terms of momentum, this pole sits at $|\mathbf{k}| \sim$ $(-i)(8 \mathrm{MeV})$. The unnaturally low energies at which this and the deuteron pole reside are an experimental fact. Such threshold states can only result from a fine-tuning between long and short distance physics, and so their existence can never be understood using naive dimensional analysis (NDA).

In an EFT such fine-tuned bound states result in operators that are naively irrelevant (in the RG sense), playing a crucial role. Consequently, power counting based on the NDA we are familiar with in the zero- and one-nucleon sectors 13 does not apply. In fact, as is discussed below, the two-nucleon system is very close to a non-trivial fixed point in the RG-flow. Another way to see this is to remind ourselves of something obvious: the theory is nonperturbative. Nuclei are bound states of nucleons and so will never arise at any finite order in perturbation theory. Unlike the baryon-number zero and one sectors, the

${ }^{c}$ Strictly speaking this is not true, since the mass difference between the nucleon and the $\Delta$ is $M_{\Delta}-M=232 \mathrm{MeV}$.

${ }^{d}$ Here we use the nuclear spectroscopic notation ${ }^{2 S+1} L_{J}$, where $S$ is the total spin of the $N N$ system, $J$ denotes its total angular-momentum, and $L$ denotes its orbital angular momentum. 
EFT appropriate to nuclei must admit non-perturbative structure somehow and somewhere. Thus, an infinite number of loop-diagrams must enter at the same order in the EFT expansion.

\subsection{Low-Energy NN Scattering Data and Unusually Large Length Scales}

Over the years there have been literally hundreds of experiments performed to measure $N N$ cross-sections and polarization observables below the pionproduction threshold. In 1993 the Nijmegen group performed a phase-shift analysis (PSA) of these experiments 2. This analysis summarizes all of this data in terms of the phase shifts and mixing parameters that describe scattering in the different spin-angular momentum channels. Clearly any theory which reproduces these phase shifts will also reproduce the data. Conversely, although the mapping from data to phase shifts is not mathematically unique, the Nijmegen group's imposition of "reasonable" physical constraints apparently makes it quite difficult to obtain phase shifts which differ radically from those found in Ref.2. We will always compare the EFT phase shifts and mixing parameter(s) with the Nijmegen PSA and not directly with data.

At low energies the $N N$ cross-section is dominated by S-wave scattering. Let us recall the behavior of the phase shift in a strongly-interacting system. Firstly, the S-matrix of two non-relativistic nucleons scattering in the ${ }^{1} S_{0}$ channel with center-of-mass momentum $|\mathbf{k}|=\sqrt{M T_{\text {lab }} / 2}$ is

$$
S=e^{i 2 \delta}=1+\frac{2 i|\mathbf{k}|}{|\mathbf{k}| \cot \delta-i|\mathbf{k}|}=1+i \frac{|\mathbf{k}| M}{2 \pi} \mathcal{A},
$$

where $\delta$ is the phase shift, and $T_{\text {lab }}$ is the kinetic energy of the incoming nucleon in the laboratory frame. We use a normalization such that explicit computation of Feynman diagrams, in an arbitrary frame, gives the non-relativistic amplitude $\mathcal{A}$. From elementary scattering theory, $|\mathbf{k}| \cot \delta$ is an analytic function of external kinetic energy with a radius of convergence bounded by the nearest t-channel singularity. In the case of $N N$ scattering this is the pion cut, which occurs at $|\mathbf{k}|=m_{\pi} / 2$. Within this radius of convergence we may use the expansion:

$$
|\mathbf{k}| \cot \delta=-\frac{1}{a}+\frac{1}{2} r_{0}|\mathbf{k}|^{2}+r_{1}|\mathbf{k}|^{4}+\ldots
$$

While Eq. (4) corresponds to expanding about $|\mathbf{k}|=0,|\mathbf{k}| \cot \delta$ can, of course, be expanded about any point within the region of convergence. In general, the size of the coefficients $r_{0}$ (the effective range) and $r_{1}$ (the shape parameter) is determined by the range of the interaction between the nucleons, which is 
$\sim 1 / m_{\pi}$ 14, 15. One might also expect the scattering length $a$ to be "natural" in this sense. However, $a$ is not constrained by the range of the underlying interaction, and, as we have already discussed, the scattering lengths for $N N$ scattering in both the ${ }^{1} S_{0}$ and ${ }^{3} S_{1}$ channel are significantly larger than $1 / m_{\pi}$. In the ${ }^{1} S_{0}$ channel the empirical values of the parameters $a, r_{0}$, and $r_{1}$ are

$$
a=-23.714 \mathrm{fm}, \quad r_{0}=2.73 \mathrm{fm}, \quad r_{1}=-0.48 \mathrm{fm}^{3} .
$$

We could also expand about $|\mathbf{k}|=0$ in the spin-triplet S-wave channel, the ${ }^{3} S_{1}$. However, since the deuteron is present in this partial wave it is convenient to instead make the expansion of $|\mathbf{k}| \cot \delta$ about the bound-state pole position 11, 22. In fact, if one truncates the expansion in Eq. (何) at order $|\mathbf{k}|^{2}$, it is straightforward to relate the location of the pole, $|\mathbf{k}|=i \gamma$, to the ${ }^{3} S_{1}$ scattering length and effective range,

$$
\gamma=\frac{1}{r_{0}}\left[1-\sqrt{1-\frac{2 r_{0}}{a}}\right] .
$$

Neglecting relativistic effects, and expanding around the deuteron pole, $|\mathbf{k}|=$ $i \gamma$ one obtains:

$$
|\mathbf{k}| \cot \delta=-\gamma+\frac{1}{2} \rho_{d}\left(|\mathbf{k}|^{2}+\gamma^{2}\right)+w_{2}\left(|\mathbf{k}|^{2}+\gamma^{2}\right)^{2}+\ldots,
$$

with 16 :

$$
\begin{gathered}
\gamma=45.7025 \mathrm{MeV}=(4.318946 \mathrm{fm})^{-1} \\
\rho_{d}=1.764 \mathrm{fm}, \quad w_{2}=0.389 \mathrm{fm}^{3}
\end{gathered}
$$

Finally, we should mention that since the $N N$ interaction is not spherically symmetric it mixes states of different orbital angular momentum. In particular, the deuteron is not solely a bound state in the ${ }^{3} S_{1}$ channel, but instead represents a bound state in the coupled ${ }^{3} S_{1}-{ }^{3} D_{1}$ channels. Below we shall show that this mixing effect is sub-leading in the low-energy $N N$ EFT, but still numerically significant. A more detailed discussion of this mixing is presented below.

${ }^{e}$ Throughout, we quote only the strong-interaction part of the phase shift and give the parameters in the $n p$ channel. There are corrections for charge-independence breaking and charge-symmetry breaking which shift the value of both $a$ and $r_{0}$ if one considers $n n$ or $p p$ scattering. 


\subsection{You Can't Choose Your Neighbors: Living near a Fixed Point}

We now begin our attempts to build an EFT which reproduces this low-energy $N N$ data. The location of the deuteron pole and other low-energy $N N$ observables are input for the EFT, and so it might seem that such an EFT can accomplish very little. However, as explained above, a low-energy pole in the scattering amplitude necessarily involves short-distance physics, and so the EFT must have this information as input. It simply cannot be expected to yield it as output. (See Ref. 17 for an early and explicit implementation of this idea.) Putting the bound state in "by hand" in this way does not vitiate the ability to make predictions with the EFT. Once the physics of the lowenergy pole is included in the theory we can use symmetries to predict other observables, such as the cross-section for $n p \rightarrow d \gamma$.

The extreme situation where $\gamma=0$ corresponds to a bound state at threshold. If, in addition, the mass scale of the potential is much greater than the incident center-of-mass momentum the theory is approximately scale invariant. This leads to a somewhat peculiar EFT, which exhibits a non-trivial fixed point.

Our goal is to recover such an $N N$ scattering amplitude from a Lagrange density with local four-nucleon momentum-independent operators alone:

$$
\mathcal{L}=N^{\dagger}\left[i D_{0}+\frac{\mathbf{D}^{2}}{2 M}\right] N-C_{0}\left(N^{T} P N\right)^{\dagger}\left(N^{T} P N\right),
$$

where $P$ is a spin and isospin projector. Note that in the normal $\chi \mathrm{PT}$ counting the contribution of the $\mathbf{D}^{2}$ term is suppressed. However, in a non-relativistic two-body bound state its contribution is of the same order as the contribution from the $i D_{0}$ term in Eq. (9). In other words, the kinetic energy of the $N N$ bound state is the same order as both its potential energy and its total energy. Further, the size of the interaction term can be estimated by NDA,

$$
C_{0} \sim \frac{1}{M \Lambda},
$$

where $\Lambda$ is the "high" scale in this theory, which is $\sim m_{\pi}$.

The Lagrange density in Eq. (9) is sufficiently simple that the sum of all Feynman diagrams contributing to $N N$ scattering, shown in Fig. 2, can be found exactly. Note that in constructing the $N N$ amplitude in this way, contributions from an irrelevant operator are being resummed. If the size of $C_{0}$ were in fact given by NDA, as in Eq. (10), each additional loop in the bubble sum would be suppressed by one power of $k / \Lambda$. Thus, without any further 
work we see that the NDA scaling of Eq. (10) cannot produce a low-energy $N N$ bound state.

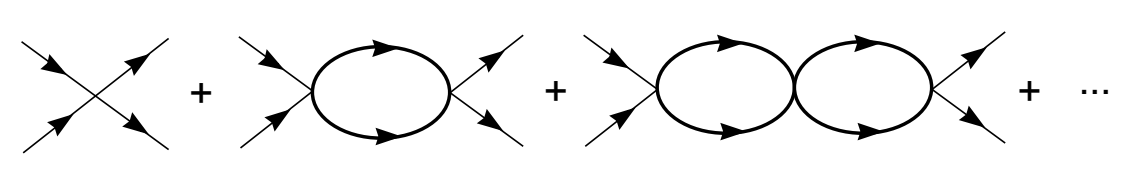

Figure 2: Feynman diagrams contributing to the scattering amplitude from the Lagrange density in Eq. (9).

If, in spite of this, we proceed to sum the bubble chain depicted in Fig. 2 we obtain an amplitude:

$$
\mathcal{A}=-\left[C_{0}+C_{0} I_{0} C_{0}+C_{0} I_{0} C_{0} I_{0} C_{0}+\ldots\right]=\frac{-1}{\frac{1}{C_{0}}-I_{0}}
$$

where $I_{0}$ is the momentum-independent single-loop integral, which in the $N N$ center-of-mass has the form:

$$
\begin{aligned}
I_{0} & =-i\left(\frac{\mu}{2}\right)^{4-n} \int \frac{\mathrm{d}^{n} q}{(2 \pi)^{n}}\left(\frac{i}{E+q_{0}-\mathbf{q}^{2} / 2 M+i \epsilon}\right)\left(\frac{i}{-q_{0}-\mathbf{q}^{2} / 2 M+i \epsilon}\right) \\
& =\left(\frac{\mu}{2}\right)^{4-n} \int \frac{\mathrm{d}^{(n-1)} \mathbf{q}}{(2 \pi)^{(n-1)}}\left(\frac{1}{E-\mathbf{q}^{2} / M+i \epsilon}\right) \\
& =-M\left(-|\mathbf{k}|^{2}-i \epsilon\right)^{\left(\frac{n-3}{2}\right)} \Gamma\left(\frac{3-n}{2}\right) \frac{\left(\frac{\mu}{2}\right)^{4-n}}{(4 \pi)^{\left(\frac{n-1}{2}\right)}},
\end{aligned}
$$

with $|\mathbf{k}|=\sqrt{M E}$ the three-momentum of each nucleon in the center-of-mass frame. Note that this $I_{0}$ is linearly divergent, and so we have evaluated it in $n$ space-time dimensions. In order to go further, a subtraction scheme must be specified; different subtraction schemes amount to a reshuffling between contributions from the vertices and contributions from the the UV part of the loop integration. The usual $\overline{\mathrm{MS}}$ scheme corresponds to taking the $n \rightarrow 4$ limit of the expression in Eq. (12), obtaining:

$$
I_{0} \stackrel{\overline{\mathrm{MS}}}{\longrightarrow}-\left(\frac{M}{4 \pi}\right) i|\mathbf{k}| .
$$

In this case each successive term in the bubble-sum in Eq. (11) involves the product $C_{0} M|\mathbf{k}|$, and so, in order to produce a bound state of momentum $\gamma$ it 
is necessary to have the coefficient $C_{0}$ scale as $C_{0} \sim 1 /(M \gamma)$. This unnatural scaling in $\overline{\mathrm{MS}}$, and the problems it leads to, were first discussed by Kaplan, Savage, and Wise 18. $C_{0}$ is simply unnaturally large: in fact it is ill-defined for the case of a threshold bound state.

There were many discussions as to how to deal with this problem in EFT, with some advocating the use of cutoff regularization, instead of dimensional regularization, so that the ynnaturally large value of $C_{0}$ would not

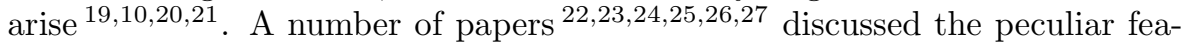
tures which arise in this EFT when one naively implements different regularization schemes. Ultimately though, the issue here was not associated with the use of a specific regularization scheme. Instead, thesplution to this difficulty lay in the development of a new power counting 28,29.30.31.32. Subsequently it was shown how to implement this power counting on a diagram-by-diagram basis using a general subtraction procedure called Power Divergence Subtraction 3031 (PDS).

In PDS both the $n=4$ and $n=3$ poles are subtracted from each bubble. The logarithmic divergence in $n=3$-which corresponds to the power-law divergence in $n=4$-is then included in the expression for the bubble. PDS thereby keeps track of linear divergences (see Ref. 33 for extensions of this idea). This allows for a fine-tuning between the coefficient $C_{0}$ and the linear divergence which produces a shallow two-body bound state. In PDS, the integral $I_{0}$ in Eq. (12) is defined to be

$$
I_{0} \stackrel{\mathrm{PDS}}{\longrightarrow}-\left(\frac{M}{4 \pi}\right)(\mu+i|\mathbf{k}|) .
$$

A similar expression isphtained with a momentum cut-offeg or with a momentumspace subtraction 442735. If PDS is employed the bubble sum (11) yields

$$
\mathcal{A}=-\frac{4 \pi}{M} \frac{1}{\frac{4 \pi}{M C_{0}}+\mu+i|\mathbf{k}|} .
$$

Matching to the leading-order ERT amplitude in the ${ }^{3} S_{1}$ channel, given by Eq. (3) with $|\mathbf{k}| \cot \delta=-\gamma$, we find

$$
\frac{4 \pi}{M C_{0}}=\gamma-\mu .
$$

In the limit of vanishing binding energy, it is then clear that

$$
\mu \frac{d}{d \mu}\left[\mu C_{0}(\mu)\right]=0 .
$$


At the fixed point the theory is conformally invariant (in the two-body sector) 36. Therefore, in order for the four-nucleon interaction in Eq. (9) to generate a system with a shallow bound state (i.e. $\gamma \sim 0$ ), $C_{0}$ must be near a non-trivial fixed point, as given by Eq. (17). The existence of this non-trivial fixed point 31 is independent of the particular regularization and renormalization scheme chosen 37 .

The explicit introduction of the subtraction scale $\mu$ into the expression for the amplitude results in $C_{0}$ having its scale set by $\mu$ and not by $\gamma$. Ultimately all of the couplings associated with higher-dimensional two-nucleon operators also scale with $\mu$, as well as with the underlying scale, $\Lambda$. A useful powercounting emerges if $\mu$ is taken to be of order the externalmomentum $-\mathbf{k} \mid$. This leads to a regulator-independent power-counting 32.39.38.29.31,34.27.35, 37, known as Q-counting. In Q-counting $\gamma$ and the external momentum $\mathbf{k}$ f] are both denoted by $Q$, and Feynman diagrams are analyzed in terms of the power of this parameter that they carry. The result is an expansion in powers of $Q / \Lambda$, where $\Lambda$ is the underlying scale which sets the size of the higher-order coefficients in the effective-range expansion, i.e. $\Lambda \sim 1 / r_{e} \sim m_{\pi}$. Q-counting is regulator-independent, but if PDS is used then each diagram has a definite order in the Q-expansion and gauge invariance is explicitly preserved. These advantages greatly simplify higher-order calculations of processes other than $N N$ scattering 30.39 .

This completes the discussion of the Lagrangian in Eq. (9) in the $N N$ system. Now we generalize this analysis to the most general $\mathrm{S}$-wave $N N$ interaction that is consistent with Galilean invariance. In this case the sum of all graphs contributing to $N N \rightarrow N N$ can be determined exactly if PDS is used to define the divergent loops. Suppose the tree-level amplitude for $N N$ scattering is

$$
\mathcal{A}_{\text {tree }}=-\left(\frac{\mu}{2}\right)^{4-n} \sum_{r=0}^{\infty} C_{2 r}(\mu)|\mathbf{k}|^{2 r} .
$$

Here the coefficients $C_{2 r}(\mu)$ are linear combinations of couplings in the Lagrangian for operators with $2 r$ gradients which contribute to S-wave scattering. If this vertex - instead of the vertex $C_{0}$-is used for each interaction in the bubble chain of Fig. 2 the resultant amplitude is

$$
\frac{1}{\mathcal{A}}=-\frac{1}{\sum C_{2 r}(\mu)|\mathbf{k}|^{2 r}}+i \frac{M}{4 \pi}(\mu+i|\mathbf{k}|) \text {. }
$$

The $\mu$-independence of this amplitude gives RG flow equations for each of the coefficients $C_{2 r}(\mu)$. Matching to the ERT amplitude, Eqs. (3) and (7), for $N N$

\footnotetext{
${ }^{f}$ Consequently $\mu$ is also denoted by $Q$.
} 
scattering, requires that for $\mu \sim \gamma \sim Q$ the couplings $C_{2 r}(\mu)$ scale as

$$
C_{2 r}(\mu) \sim \frac{4 \pi}{M \Lambda^{r} Q^{r+1}} .
$$

Thus the $r$ th term in the sum for $\mathcal{A}_{\text {tree }}, C_{2 r} k^{2 r}$, has a Q-counting order of $Q^{r-1}$. The counting rules for this low-energy effective theory of $N N$ scattering are then as follows:

- Each nucleon propagator scales as $1 / Q^{2}$;

- Each loop scales as $Q^{5}$;

- Each vertex $C_{2 r} k^{2 r}$ scales as $Q^{r-1}$.

In this way each $N N$ loop contributes one factor of $Q$, but the vertex $C_{0}$ scales as $Q^{-1}$, and so, by construction, the leading-order contribution in this EFT, is the complete bubble-chain shown in Fig. 2, with $C_{0}$ operators at all the four-nucleon vertices. The leading-order amplitude, $\mathcal{A}^{(-1)}$, is then given by Eq. (15). Contributions to the $N N$ amplitude which scale as higher powers of $Q$ come from perturbative insertions of derivative interactions, $C_{2 r} k^{2 r}, r \geq 1$, but these are always "dressed" by the leading-order (LO) amplitude $\mathcal{A}^{(-1)}$, since the product of $\mathcal{A}^{(-1)}$ with a loop is of order $Q^{0}$.

\subsection{Low-Energy Nucleon-Nucleon Interactions: ERT $\rightarrow \operatorname{EFT}(\nrightarrow)$}

The previous section contains all that is needed in order to set up the EFT for low-energy $N N$ interactions. We will call this theory $\operatorname{EFT}(\AA)$ since it does not contain pions as explicit degrees of freedom. EFT $(\not)$ reproduces the ERT $N N$ scattering amplitude $\mathcal{A}$ order-by-order in a $Q$-expansion. If this was all that it did, then $\operatorname{EFT}(\hbar)$ would represent no more than ERT. However, $\operatorname{EFT}(\Uparrow)$ 's power becomes apparent when external probes of the $N N$ system are considered. One of the very interesting results obtained in $\operatorname{EFT}(\not)$ is that ERT generally gives the LO contribution to observables where the $N N$ system is coupled to low-momentum external probes, such as photons and weak gauge bosons 3933 . This justifies the use of ERT to estimate, for instance, the magnetic moment of the deuteron. However, ERT computations of deuteron properties are not always successful, since ERT only gives the LO contribution to these observables, and subleading corrections can be numerically significant. Operators in $\operatorname{EFT}(\hbar)$ exist which are absent in ERT, and these operators contribute to electroweak observables. Including such operators allows $\operatorname{EFT}(\hbar)$ to produce closed form, analytic expressions for electroweak processes such as $n p \rightarrow d \gamma$ and $\nu d \rightarrow \nu d$ which are accurate at the $1 \%$ level. 


\subsection{NN Scattering in the ${ }^{1} S_{0}$ Channel}

Nucleon-nucleon scattering in the ${ }^{1} S_{0}$ channel is described by the Lorentzinvariant one-body Lagrange density

$$
\mathcal{L}_{1}=N^{\dagger}\left[i D_{0}+\frac{\mathbf{D}^{2}}{2 M}-\frac{D_{0}^{2}}{2 M}\right] N
$$

and the two-body Lagrange density - up to $\mathrm{N}^{2} \mathrm{LO}$ :

$$
\begin{aligned}
\mathcal{L}_{2} & ={ }^{\pi} C_{0}^{\left({ }^{1} S_{0}\right)}\left(N^{T} \bar{P}^{a} N\right)^{\dagger}\left(N^{T} \bar{P}^{a} N\right) \\
& -{ }^{\pi} C_{2}^{\left(1 S_{0}\right)} \frac{1}{2}\left[\left(N^{T} \bar{P}^{a} N\right)^{\dagger}\left(N^{T} \mathcal{O}_{2}^{a} N\right)+\text { h.c. }\right] \\
& -{ }^{\pi} C_{4}^{\left({ }^{1} S_{0}\right)}\left(N^{T} \mathcal{O}_{2}^{a} N\right)^{\dagger}\left(N^{T} \mathcal{O}_{2}^{a} N\right) \\
& -{ }^{\pi} \tilde{C}_{4}^{\left({ }^{1} S_{0}\right)} \frac{1}{2}\left[\left(N^{T} \bar{P}^{a} N\right)^{\dagger}\left(N^{T} \mathcal{O}_{4}^{a} N\right)+\text { h.c. }\right],
\end{aligned}
$$

where $\bar{P}^{a}$ is the spin-isospin projector for this channel: $[J=0, I=1]$

$$
\bar{P}^{a} \equiv \frac{1}{\sqrt{8}} \sigma_{2} \tau_{2} \tau^{a} \quad, \quad \operatorname{Tr}\left[\bar{P}^{a \dagger} \bar{P}^{b}\right]=\frac{1}{2} \delta^{a b},
$$

and where the derivative operators are

$$
\begin{aligned}
& \mathcal{O}_{2}^{a}=-\frac{1}{4}\left[\bar{P}^{a} \overrightarrow{\mathbf{D}}^{2}+\overleftarrow{\mathbf{D}}^{2} \bar{P}^{a}-2 \overleftarrow{\mathbf{D}} \bar{P}^{a} \overrightarrow{\mathbf{D}}\right] \\
& \mathcal{O}_{4}^{a}=+\frac{1}{16}\left[\bar{P}^{a} \overrightarrow{\mathbf{D}}^{4}-4 \overleftarrow{\mathbf{D}} \bar{P}^{a} \overrightarrow{\mathbf{D}}^{3}+6 \overleftarrow{\mathbf{D}}^{2} \bar{P}^{a} \overrightarrow{\mathbf{D}}^{2}-4 \overleftarrow{\mathbf{D}}^{3} \bar{P}^{a} \overrightarrow{\mathbf{D}}+\overleftarrow{\mathbf{D}}^{4} \bar{P}^{a}\right]
\end{aligned}
$$

The subscript on the coefficient denotes the number of derivatives in the operator, and $\overrightarrow{\mathbf{D}}^{2}=\overrightarrow{\mathbf{D}}^{j} \overrightarrow{\mathbf{D}}^{j}$. We have employed the covariant derivative, $D_{\mu}=\left(\partial_{0}+i e Q A_{0},-\nabla+i e Q \mathbf{A}\right)$, in defining the two and four derivative operators in Eq. (24) where $A_{\mu}$ is the electromagnetic field and $Q$ is the electromagnetic charge matrix. Contributions to the Lagrange density in Eq. (22) including more than four derivatives can be found in Refs. 49.41 . Hereafter relativistic corrections to observables will not be included in expressions, unless explicitly stated otherwise. It is always straightforward to include the third term in Eq. (21), which generates these corrections, in perturbation theory 39.

At LO the scattering amplitude found from Eqs. (21) and (22) is that shown in Eq. (15). Matching this to the ${ }^{1} S_{0}$ amplitude from ERT, Eq. (何), gives

$$
{ }^{\pi} C_{0}^{\left({ }^{1} S_{0}\right)}(\mu)=-\frac{4 \pi}{M} \xi^{\left({ }^{1} S_{0}\right)}(\mu) \quad \text { with } \quad \xi^{\left({ }^{1} S_{0}\right)}(\mu)=\frac{1}{\mu-1 / a^{\left(1 S_{0}\right)}} .
$$


Note that in Q-counting $r_{0} \sim 1 / \Lambda$, while $1 / a, \mathbf{k} \sim Q$. Using this counting and expanding the ${ }^{1} S_{0}$ ERT amplitude defined by (3) and (仼) in powers of $Q / \Lambda$ we can match to the NLO amplitude in $\operatorname{EFT}(\hbar)$. This yields:

$$
{ }^{\pi} C_{2}^{\left({ }^{1} S_{0}\right)}(\mu)=\frac{4 \pi}{M} \frac{r_{0}}{2}\left(\xi^{\left({ }^{1} S_{0}\right)}(\mu)\right)^{2} .
$$

Carrying out the same procedure at $\mathrm{N}^{2} \mathrm{LO}$, the matching gives:

$$
{ }^{\pi} C_{4}^{\left({ }^{1} S_{0}\right)}(\mu)+{ }^{\pi} \tilde{C}_{4}^{\left({ }^{1} S_{0}\right)}(\mu)=-\frac{4 \pi}{M}\left(\xi^{\left({ }^{1} S_{0}\right)}(\mu)\right)^{3}\left[\frac{1}{4}\left(r_{0}^{\left({ }^{1} S_{0}\right)}\right)^{2}-\frac{r_{1}^{\left({ }^{1} S_{0}\right)}}{\xi^{\left({ }^{1} S_{0}\right)}(\mu)}\right],
$$

It is interesting to note that only the sum $\left.{ }^{\pi} C_{4}^{\left({ }^{1} S_{0}\right)}+{ }^{\pi} \tilde{C}_{4}^{(1} S_{0}\right)$ can be constrained by $N N$ scattering. RG scaling 31,30 of the operators in Eq. (22) indicates that while the contribution from ${ }^{\pi} C_{4}^{\left({ }^{1} S_{0}\right)}$ is $\mathrm{N}^{2} \mathrm{LO}$, the contribution from ${ }^{\pi} \tilde{C}_{4}^{\left({ }^{1} S_{0}\right)}$ is $\mathrm{N}^{3} \mathrm{LO}$. The time-ordered product of two ${ }^{\star} C_{2}^{\left({ }^{1} S_{0}\right)}$ operators does not induce the momentum structure of the ${ }^{\star} \tilde{C}_{4}^{\left({ }^{1} S_{0}\right)}$ operator. However, inelastic processes can be sensitive to different combinations of ${ }^{\pi} C_{4}^{\left({ }^{1} S_{0}\right)}$ and ${ }^{\pi} \tilde{C}_{4}^{\left({ }^{1} S_{0}\right)}$.

The convergence of $\operatorname{EFT}(\hbar)$ can be tested by an examination of the behavior of the phase shift $\delta^{\left({ }^{1} S_{0}\right)}$ order by order in the $Q$-expansion. The results are shown in Fig. 3. It is clear from Fig. 3 that in the ${ }^{1} S_{0}$ channel the expansion must be carried out to $\mathrm{N}^{4} \mathrm{LO}$ or higher to describe the strong interactions for $|\mathbf{k}| \lesssim 100 \mathrm{MeV}$. Also, it is clear that $\operatorname{EFT}(\not)$ will not be valid for $|\mathbf{k}| \gtrsim 150 \mathrm{MeV}$. At these momenta the full $|\mathbf{k}| \cot \delta$ description of the phase shift starts to deviate from the observed phase shift. Given the location of the t-channel pion-exchange cut in the $N N$ amplitude this deviation is expected.

\subsection{NN Scattering in the Coupled ${ }^{3} S_{1}-{ }^{3} D_{1}$ Channel}

Scattering in the $J=1$ channel is somewhat more complicated because both $L=0\left({ }^{3} S_{1}\right)$ and $L=2\left({ }^{3} D_{1}\right)$ states contribute. The S-matrix describing scattering in the coupled channel $J=1$ system is written as

$$
S=\left(\begin{array}{cc}
e^{i 2 \delta_{0}} \cos 2 \bar{\varepsilon}_{1} & i e^{i\left(\delta_{0}+\delta_{2}\right)} \sin 2 \bar{\varepsilon}_{1} \\
i e^{i\left(\delta_{0}+\delta_{2}\right)} \sin 2 \bar{\varepsilon}_{1} & e^{i 2 \delta_{2}} \cos 2 \bar{\varepsilon}_{1}
\end{array}\right),
$$

where we use the "barred" parameterization of Ref. 42 , which was also used in Ref. 43. It will follow naturally from the $\operatorname{EFT}(\hbar)$ that $\bar{\varepsilon}_{1}$ is supressed by $Q^{2}$ compared with $\delta_{0}^{(0)}$, and therefore, up to $\mathrm{N}^{4} \mathrm{LO}$, the ${ }^{3} S_{1}-{ }^{3} S_{1}$ element of this 


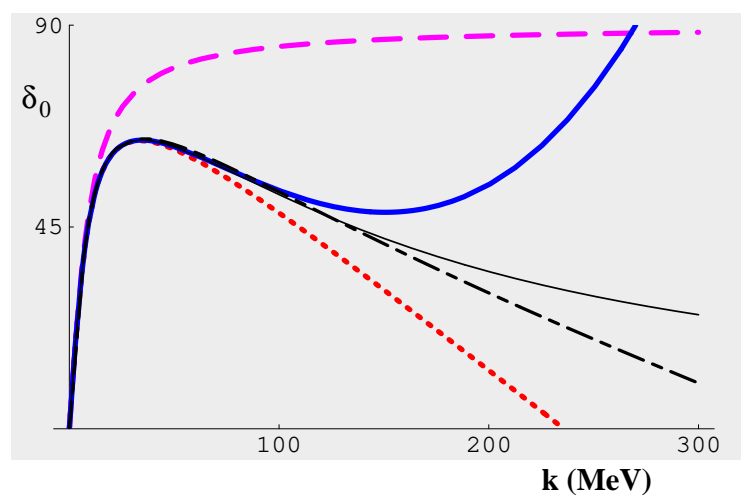

Figure 3: The phase shift $\delta^{\left({ }^{1} S_{0}\right)}$ as a function of the center of mass momentum $|\mathbf{k}|$. The dashed curve corresponds to the $\mathrm{LO}$ result, the dotted curve is the result at $\mathrm{N}^{2} \mathrm{LO}$, the thick solid curve corresponds to $\mathrm{N}^{4} \mathrm{LO}$. The thin solid curve corresponds to the phase shift from ERT, and the dot-dashed curve are the phases of the Nijmegen PSA.

matrix may be written as:

$$
S_{00}=e^{i 2 \delta_{0}}=1+\frac{2 i}{\cot \delta_{0}-i} .
$$

The phase shift $\delta_{0}$ has an expansion in powers of $Q, \delta_{0}=\delta_{0}^{(0)}+\delta_{0}^{(1)}+\delta_{0}^{(2)}+\ldots$, where the superscript denotes the order in the $Q$ expansion.

Now, taking the expressions in Eqs. (3) and (7), and expanding in powers of $k / \Lambda$ and $\gamma / \Lambda$, we obtain the LO and NLO contributions to $\mathcal{A}$, which are

$$
\mathcal{A}^{(-1)}=\frac{4 \pi}{M} \frac{1}{\gamma+i|\mathbf{k}|}, \quad \mathcal{A}^{(0)}=\frac{4 \pi}{M} \frac{Z_{d}-1}{\gamma+i|\mathbf{k}|},
$$

where $Z_{d}$ is the residue of the $N N$ scattering amplitude at the bound-state pole. In this way, the position and residue of the deuteron pole are correct in the NLO amplitude. In terms of the parameters $\gamma$ and $\rho_{d}, Z_{d}$ is

$$
Z_{d}=\frac{1}{1-\gamma \rho_{d}}
$$

Consequently $Z_{d}-1$ is of order $Q . Z_{d}$ is also related to the asymptotic Sstate normalization of the deuteron wave function by $A_{S}^{2}=2 \gamma Z_{d}$. Using the Nijmegen PSA value for $A_{S}$ we get $Z_{d}=1.69$.

Forming the logarithm of both sides of Eq. (29), using Eqs. (3) and (30), and expanding in powers of $Q$, it is straightforward to obtain the LO and NLO 
results

$$
\delta_{0}^{(0)}(|\mathbf{k}|)=\pi-\tan ^{-1}\left(\frac{|\mathbf{k}|}{\gamma}\right), \quad \delta_{0}^{(1)}(|\mathbf{k}|)=-\frac{Z_{d}-1}{2}|\mathbf{k}|
$$

which are shown in Fig. 1 . The $\mathrm{N}^{2} \mathrm{LO}$ piece of the phase shift, $\delta_{0}^{(2)}$, also

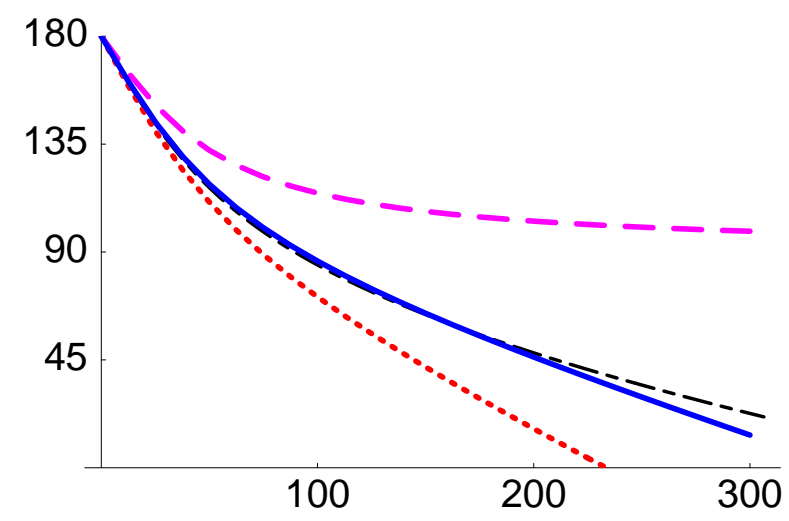

Figure 4: The phase shift $\delta_{0}$ as a function of the center of mass momentum $|\mathbf{k}|$. The dashed curve corresponds to $\delta_{0}^{(0)}$, the dotted curve corresponds to $\delta_{0}^{(0)}+\delta_{0}^{(1)}$, the solid curve corresponds to $\delta_{0}^{(0)}+\delta_{0}^{(1)}+\delta_{0}^{(2)}$, and the dot-dashed curve is the Nijmegen PSA 4 .

depends only on $Z_{d}$ and $\gamma$. Up to $\mathrm{N}^{2} \mathrm{LO}$, the shape parameter term, $w_{2}$ does not contribute to the $\mathrm{S}$-wave phase shift. In fact, $w_{2}$ is also numerically small (about a factor of 5 smaller than $\rho_{d}$ ), but even if it were not, it enters the Qexpansion only at high orders. The convergence of the Q-expansion is clearly demonstrated in Fig. 化.

The Lagrange density describing scattering in the ${ }^{3} S_{1}$ channel is the same as that in the ${ }^{1} S_{0}$ channel, but the $C$ 's and the spin-isospin projectors are different. It is thus the $\mathcal{L}_{1}$ of Eq. (21) plus

$$
\begin{aligned}
\mathcal{L}_{2} & ={ }^{\pi} C_{0}^{\left({ }^{3} S_{1}\right)}\left(N^{T} P^{i} N\right)^{\dagger}\left(N^{T} P^{i} N\right) \\
& -{ }^{\pi} C_{2}^{\left({ }^{3} S_{1}\right)} \frac{1}{2}\left[\left(N^{T} P^{i} N\right)^{\dagger}\left(N^{T} \mathcal{O}_{2}^{i} N\right)+\text { h.c. }\right] \\
& -{ }^{\pi} C_{4}^{\left({ }^{3} S_{1}\right)}\left(N^{T} \mathcal{O}_{2}^{i} N\right)^{\dagger}\left(N^{T} \mathcal{O}_{2}^{i} N\right) \\
& -{ }^{\pi} \tilde{C}_{4}^{\left({ }^{3} S_{1}\right)} \frac{1}{2}\left[\left(N^{T} P^{i} N\right)^{\dagger}\left(N^{T} \mathcal{O}_{4}^{i} N\right)+\text { h.c. }\right],
\end{aligned}
$$


where $P^{i}$ is now the spin-isospin projector for the $[J=1, I=0]$ channel,

$$
P^{i} \equiv \frac{1}{\sqrt{8}} \sigma_{2} \sigma^{i} \tau_{2}, \quad \operatorname{Tr}\left[P^{i \dagger} P^{j}\right]=\frac{1}{2} \delta^{i j},
$$

and where the derivative operators are

$$
\begin{aligned}
& \mathcal{O}_{2}^{i}=-\frac{1}{4}\left[P^{i} \overrightarrow{\mathbf{D}}^{2}+\overleftarrow{\mathbf{D}}^{2} P^{i}-2 \overleftarrow{\mathbf{D}} P^{i} \overrightarrow{\mathbf{D}}\right] \\
& \mathcal{O}_{4}^{i}=+\frac{1}{16}\left[P^{i} \overrightarrow{\mathbf{D}}^{4}-4 \overleftarrow{\mathbf{D}} P^{i} \overrightarrow{\mathbf{D}}^{3}+6 \overleftarrow{\mathbf{D}}^{2} P^{i} \overrightarrow{\mathbf{D}}^{2}-4 \overleftarrow{\mathbf{D}}^{3} P^{i} \overrightarrow{\mathbf{D}}+\overleftarrow{\mathbf{D}}^{4} P^{i}\right]
\end{aligned}
$$

Matching the amplitudes in Eq. (30) to the Q-expanded amplitude generated by the Lagrangian in Eqs. (21) and (33), gives 33 , up to NLO

$$
\begin{aligned}
{ }^{\pi} C_{0,-1}^{\left({ }^{3} S_{1}\right)} & =-\frac{4 \pi}{M} \frac{1}{(\mu-\gamma)}, \quad{ }^{\pi} C_{0,0}^{\left({ }^{3} S_{1}\right)}=\frac{2 \pi}{M} \frac{\gamma\left(Z_{d}-1\right)}{(\mu-\gamma)^{2}}, \\
{ }^{\pi} C_{2,-2}^{\left({ }^{3} S_{1}\right)} & =\frac{2 \pi}{M} \frac{Z_{d}-1}{\gamma(\mu-\gamma)^{2}} .
\end{aligned}
$$

Here, the coefficients themselves have been expanded as a power series in $Q$, e.g. ${ }^{\pi} C_{0}^{\left({ }^{3} S_{1}\right)}={ }^{\pi} C_{0,-1}^{\left({ }^{3} S_{1}\right)}+{ }^{\pi} C_{0,0}^{\left({ }^{3} S_{1}\right)}+{ }^{\pi} C_{0,1}^{\left({ }^{3} S_{1}\right)}+\ldots$, so that the location of the deuteron pole is unchanged order-by-order in the $Q$ expansion.

Turning our attention now to the off-diagonal elements of the S-matrix in Eq. (28), the $\bar{\varepsilon}_{1}$ parameter defines the amount of mixing between the ${ }^{3} S_{1}$ and ${ }^{3} D_{1}$ channels. It has a momentum expansion in terms of the constants $E_{1}^{(2)}$ and $E_{1}^{(4)}$,

$$
\bar{\varepsilon}_{1}=E_{1}^{(2)} \frac{|\mathbf{k}|^{3}}{\sqrt{|\mathbf{k}|^{2}+\gamma^{2}}}+E_{1}^{(4)} \frac{|\mathbf{k}|^{5}}{\sqrt{|\mathbf{k}|^{2}+\gamma^{2}}}+\ldots
$$

$E_{1}^{(2)}$ and $E_{1}^{(4)}$ are fit to the Nijmegen PSA 4 , and are found to be $E_{1}^{(2)}=$ $0.386 \mathrm{fm}^{2}$ and $E_{1}^{(4)}=-2.800 \mathrm{fm}^{4}$. As far as the power counting is concerned it is important to note that the $E_{1}^{(Q)}$, s are set by physics at the scale $\Lambda$ and so contributions of order $Q^{-1}$ must vanish 4 . Thus the first term here is $O(Q)$, and the second $O\left(Q^{3}\right)$. Corrections to Eq. (37) are suppressed by two further powers of $Q$. The LO and $\mathrm{N}^{2} \mathrm{LO}$ contributions to $\bar{\varepsilon}_{1}$ are shown in Fig. 5 .

In order to calculate $\bar{\varepsilon}_{1}$ up to $\mathrm{N}^{3} \mathrm{LO}$ we only require one insertion of $\mathcal{A}_{s d}^{\text {tree }}$, dressed with the leading-order amplitude for ${ }^{3} S_{1}-{ }^{3} S_{1}$ scattering (30). The Lagrange density describing the first two orders of S-D mixing interactions is 40

$$
\mathcal{L}^{(s d)}=-\mathcal{T}_{i j, x y}^{(s d)}\left[{ }^{\pi} C_{0}^{(s d)}\left(N^{T} P^{i} N\right)^{\dagger}\left(N^{T} \mathcal{O}_{2}^{x y, j} N\right)+\right.
$$




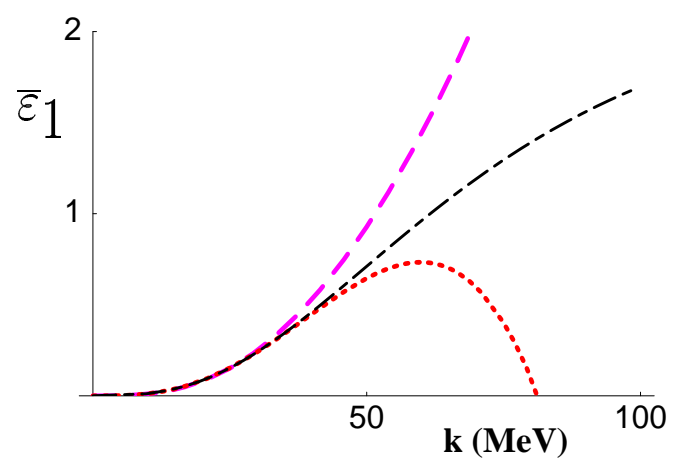

Figure 5: The mixing parameter $\bar{\varepsilon}_{1}$ as a function of the center of mass momentum $|\mathbf{k}|$. The dashed curve corresponds to $\bar{\varepsilon}^{(0)}$ and the dotted curve corresponds to $\bar{\varepsilon}_{1}^{(0)}+\bar{\varepsilon}_{\mathbb{1}}^{(1)}+\bar{\varepsilon}_{1}^{(2)}$, as determined in Eq. (37). The dot-dashed curve is the Nijmegen PSA 4 .

$$
\begin{aligned}
& \left.{ }^{\pi} C_{2}^{(s d)}\left(N^{T} \mathcal{O}_{2}^{l l, i} N\right)^{\dagger}\left(N^{T} \mathcal{O}_{2}^{x y, j} N\right)+{ }^{\pi} \tilde{C}_{2}^{(s d)}\left(N^{T} P^{i} N\right)^{\dagger}\left(N^{T} \mathcal{O}_{4}^{m m, x y, j} N\right)\right] \\
& \quad+\text { h.c., }
\end{aligned}
$$

where

$$
\begin{aligned}
\mathcal{T}_{i j, x y}^{(s d)} & =\delta_{i x} \delta_{j y}-\frac{1}{n-1} \delta_{i j} \delta_{x y} \\
\mathcal{O}_{2}^{x y, j} & =-\frac{1}{4}\left(\overleftarrow{\mathbf{D}}^{x} \overleftarrow{\mathbf{D}}^{y} P^{j}+P^{j} \overrightarrow{\mathbf{D}}^{x} \overrightarrow{\mathbf{D}}^{y}-\overleftarrow{\mathbf{D}}^{x} P^{j} \overrightarrow{\mathbf{D}}^{y}-\overleftarrow{\mathbf{D}}^{y} P^{j} \overrightarrow{\mathbf{D}}^{x}\right)
\end{aligned}
$$

The tree-level amplitude for an ${ }^{3} S_{1} \rightarrow{ }^{3} D_{1}$ transition resulting from this Lagrange density is

$$
\mathcal{A}_{s d}^{\text {tree }}=-\left({ }^{\pi} C_{0}^{(s d)}+\left[{ }^{\pi} C_{2}^{(s d)}+{ }^{\pi} \tilde{C}_{2}^{(s d)}\right] p^{2}+\ldots\right)\left[p^{i} p^{j}-\frac{1}{n-1} p^{2} \delta^{i j}\right] .
$$

Like the S-wave coefficients, the coefficients that appear in Eq. (38) have their own expansion in powers of $Q$, e.g. ${ }^{\pi} C_{0}^{(s d)}={ }^{\pi} C_{0,-1}^{(s d)}+{ }^{\pi} C_{0,0}^{(s d)}+\ldots$.

It is convenient to introduce the quantity $\eta_{s d}$. This is the asymptotic $\mathrm{D} / \mathrm{S}$ ratio of the deuteron, and may be extracted from the ${ }^{3} S_{1}-{ }^{3} D_{1}$ scattering information by analytic continuation of the amplitude to the deuteron pole. 
In fact,

$$
-2\left(\frac{\eta_{s d}}{1-\eta_{s d}^{2}}\right)=\left.\frac{\tan \left(2 \bar{\varepsilon}_{1}\right)}{\sin \left(\delta_{0}-\delta_{2}\right)}\right|_{|\mathbf{k}|=i \gamma} .
$$

The value is then found from a continuation of the Nijmegen PSA into the unphysical region: $\eta_{s d}=0.02543 \pm 0.00007$.

Matching the ${ }^{3} S_{1}-{ }^{3} D_{1}$ scattering amplitude to the Q-expansion of the off-diagonal elements of the S-matrix in Eq. (28) evaluated at the deuteron pole, gives the coefficients in Eq. (38) in terms of $\left\{\eta_{s d}, E_{1}^{(4)}\right\}$,

$$
\begin{aligned}
& { }^{\pi} C_{0,-1}^{(s d)}=-\eta_{s d} \frac{6 \sqrt{2} \pi}{M \gamma^{2}(\mu-\gamma)},{ }^{\pi} C_{0,0}^{(s d)}=\eta_{s d}\left(Z_{d}-1\right) \frac{3 \sqrt{2} \pi}{M \gamma(\mu-\gamma)^{2}} \\
& { }^{\pi} C_{2,-2}^{(s d)}+{ }^{\pi} C_{2,-2}^{(s d)}=\eta_{s d}\left(Z_{d}-1\right) \frac{3 \sqrt{2} \pi}{M \gamma^{3}(\mu-\gamma)^{2}}
\end{aligned}
$$

In this way one ensures that that all of the asymptotic physics of the deuteron state: its binding energy, and its asymptotic $\mathrm{S}$ and D-state normalizations, are reproduced in $\operatorname{EFT}(\Uparrow)$ at low orders in the $Q$-expansion.

Finally, the four-nucleon operator that first contributes to scattering in the ${ }^{3} D_{1}$ channel involves four derivatives. It is also renormalized by the operators contributing to $\bar{\varepsilon}_{1}$. Analysis of the powers of $Q$ which appear in the ${ }^{3} D_{1}$ phase shift leads us to conclude that it contributes to the observables we examine in this chapter at a much higher order than we will be concerned with, and therefore we do not consider it further.

\subsection{Processes Involving the Deuteron: Formalism}

When considering elastic or inelastic processes involving the deuteron it is convenient to define an interpolating field for the deuteron $\mathcal{D}_{i}(x)=N^{T} P_{i} N(x)$, where $P_{i}$ is the projector defined in Eq. (34). The Green's function associated with this interpolating field is then just that for the ${ }^{3} S_{1}$ channel:

$$
\int d \mathbf{x} e^{-i(E t-\mathbf{k} \cdot \mathbf{x})}\left\langle 0\left|\mathrm{~T}\left[\mathcal{D}_{i}^{\dagger}(x) \mathcal{D}_{j}(0)\right]\right| 0\right\rangle \equiv G(\bar{E}) \delta_{i j},
$$

where, by Lorentz invariance, the propagator only depends on the energy in the center of mass frame, namely $\bar{E} \equiv E-\frac{\mathbf{k}^{2}}{4 M}+\ldots$ with the ellipses referring to relativistic corrections.

The Green's function $G$ is straightforwardly related to the amplitude $\mathcal{A}$ in the ${ }^{3} S_{1}$ channel. However, some relationships between observables are clearer 
when expressed in terms of this deuteron-deuteron Green's function. Since there is a pole in this channel we may always write $G(\bar{E})$ as:

$$
G(\bar{E})=i \frac{\mathcal{Z}(\bar{E})}{\bar{E}+B}
$$

where $B$ is the deuteron binding energy and the numerator $\mathcal{Z}$ is a function which is smooth near the deuteron pole. When evaluated for $E \approx B, \mathcal{Z}$ gives the wavefunction renormalization $Z_{\psi}$. This is related to the $Z_{d}$ defined in Eq. (31) by simple factors:

$$
\mathcal{Z}(-B) \equiv Z_{\psi}=-i\left[\frac{\mathrm{d} G^{-1}(\bar{E})}{\mathrm{d} E}\right]_{\bar{E}=-B}^{-1}=-\frac{8 \pi \gamma}{M^{2}} Z_{d} .
$$

It is now useful to define " $C_{0}$-irreducible" Green's functions, since observables can be computed solely in terms of these quantities. A $C_{0}$-irreducible Green's function is the sum of all possible diagrams which do not involve the leading-order interaction ${ }^{\star} C_{0,-1}^{\left({ }^{3} S_{1}\right)}$ and contribute to a given n-point function. The irreducible deuteron-deuteron function is denoted by $\Sigma$. It has the expansion shown in Fig. 6 .

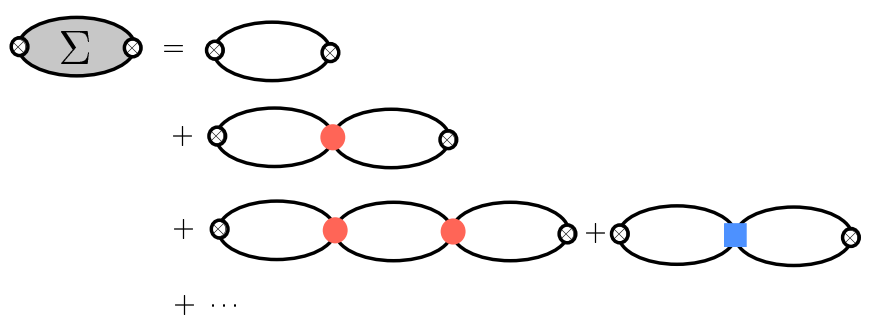

Figure 6: The perturbative expansion of $\Sigma$. The first row has the LO result, where $\otimes$ represents an insertion of the deuteron interpolating field. The second row is the NLO contribution, arising from a single insertion of the ${ }^{\pi} C_{0,0}^{\left({ }^{3} S_{1}\right)}$ and ${ }^{\pi} C_{2,-2}^{\left({ }^{3} S_{1}\right)}$ operators. The third row is the $\mathrm{N}^{2} \mathrm{LO}$ contribution, arising from two insertions of the ${ }^{\pi} C_{0,0}^{\left({ }^{3} S_{1}\right)}$ and ${ }^{\pi} C_{2,-2}^{\left({ }^{3} S_{1}\right)}$ operators, and a single insertion of the ${ }^{\pi} C_{0,1}^{\left({ }^{3} S_{1}\right)},{ }^{\pi} C_{2,-1}^{\left({ }^{3} S_{1}\right)}, \pi \tilde{C}_{4,-3}^{\left({ }^{3} S_{1}\right)}$ and ${ }^{\pi} C_{4,-3}^{\left({ }^{3} S_{1}\right)}$.

A simple consequence of this definition is that the relationship between the full $G$ and $\Sigma$ is:

$$
G=\frac{\Sigma}{1+i^{\star} C_{0,-1}^{\left(3_{1}\right)} \Sigma}
$$




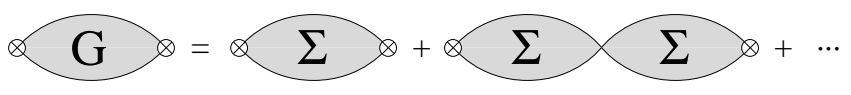

Figure 7: The expansion of of the full 2-point function $G$ in terms of the irreducible 2-point function $\Sigma$.

This can also be seen graphically in Fig. 7.

Recall that we wish to choose the coefficients ${ }^{\star} C_{0,-1}^{\left({ }^{3} S_{1}\right)},{ }^{\pi} C_{0,0}^{\left({ }^{3} S_{1}\right)}$ and ${ }^{\star} C_{2,-2}^{\left({ }^{3} S_{1}\right)}$ so as to reproduce the Green's function (44), with the function $\mathcal{Z}$ having the precise value (45) at the deuteron pole. This imposes the following conditions on $\Sigma$ :

$$
\Sigma(\bar{E}=-B)=\frac{i}{\pi^{\pi} C_{0,-1}^{\left(3 S_{1}\right)}}, \quad\left[\frac{1}{\Sigma^{2}} \frac{\mathrm{d} \Sigma}{\mathrm{d} E}\right](\bar{E}=-B)=\frac{i}{Z_{\psi}} .
$$

These conditions hold at each order of the Q-expansion, and so restrict the number of independent higher-order couplings which appear in the deuteron channel.

We are now in position to compute the matrix element of an electroweak current between two deuteron states. We first define the three-point function

$G_{i j}^{\mu}\left(\bar{E}, \bar{E}^{\prime}, \mathbf{q}\right)=\int d \mathbf{x} d \mathbf{y} e^{-i\left(E x^{0}-\mathbf{k} \cdot \mathbf{x}\right)} e^{i\left(E^{\prime} y^{0}-\mathbf{k}^{\prime} \cdot \mathbf{y}\right)}\left\langle 0\left|\mathrm{~T}\left[\mathcal{D}_{i}^{\dagger}(x) J_{e w}^{\mu}(0) \mathcal{D}_{j}(y)\right]\right| 0\right\rangle$,

where $q^{\mu}=\left(E^{\prime}-E, \mathbf{k}^{\prime}-\mathbf{k}\right)$ is the momentum transferred to the deuteron system. $G^{\mu}$ is related to the desired form factor via the LSZ formula

$$
\left\langle\mathbf{k}^{\prime}, j\left|J_{e w}^{\mu}\right| \mathbf{k}, i\right\rangle=Z_{\psi}\left[G^{-1}(\bar{E}) G^{-1}\left(\bar{E}^{\prime}\right) G_{i j}^{\mu}\left(\bar{E}, \bar{E}^{\prime}, \mathbf{q}\right)\right]_{\bar{E}, \bar{E}^{\prime} \rightarrow-B},
$$

where $G(\bar{E})$ is defined in Eq. 433). As promised, this formula may now be reexpressed in terms of $\Sigma$ and the " $C_{0}$-irreducible" 3-point function, $\Gamma^{\mu}$. Again, it is then a simple matter of definition that:

$$
G_{i j}^{\mu}\left(\bar{E}, \bar{E}^{\prime}, \mathbf{q}\right)=\frac{\Gamma_{i j}^{\mu}\left(\bar{E}, \bar{E}^{\prime}, \mathbf{q}\right) G(\bar{E}) G\left(\bar{E}^{\prime}\right)}{\Sigma(\bar{E}) \Sigma\left(\bar{E}^{\prime}\right)} .
$$

This relationship is depicted in Fig. 8. Making use of Eqs. (50), (46), (47), and (49), S-matrix elements can then be found in terms of the $C_{0}$-irreducible vertices of the theory

$$
\left\langle\mathbf{k}^{\prime}, j\left|J_{e m}^{\mu}\right| \mathbf{k}, i\right\rangle=i\left[\frac{\Gamma_{i j}^{\mu}\left(\bar{E}, \bar{E}^{\prime}, \mathbf{q}\right)}{\mathrm{d} \Sigma(\bar{E}) / \mathrm{d} E}\right]_{\bar{E}, \bar{E}^{\prime} \rightarrow-B} .
$$




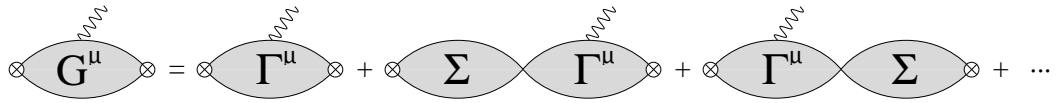

Figure 8: The expansion of full three-point function $G^{\mu}$ in terms of the irreducible twoand three-point functions $\Sigma, \Gamma^{\mu}$.

Since $\Sigma$ and $\Gamma$ both have a simple perturbative expansion in terms of Feynman graphs the right-hand side of this equation can be straightforwardly calculated to an arbitrary order in the Q-expansion, thereby providing a result for the current matrix element appearing on the left-hand side.

\section{Phenomenological Applications of $\operatorname{EFT}(\pi)$}

\subsection{Electron-Deuteron Scattering}

A deuteron with four-momentum $p^{\mu}$ and polarization vector $\epsilon^{\mu}$ is described by the state $|\mathbf{p}, \epsilon\rangle$, where the polarization vector satisfies $p_{\mu} \epsilon^{\mu}=0$. It is convenient to choose the basis polarization vectors so that in the deuteron rest frame $\epsilon_{i}^{\mu}=\delta_{i}^{\mu}$. We then write $|\mathbf{p}, i\rangle \equiv\left|\mathbf{p}, \epsilon_{i}^{\mu}\right\rangle$, and these states have the normalization condition $\left\langle\mathbf{p}^{\prime}, j \mid \mathbf{p}, i\right\rangle=(2 \pi)^{3} \delta^{3}\left(\mathbf{p}-\mathbf{p}^{\prime}\right) \delta_{i j}$. In terms of these states $|\mathbf{p}, i\rangle$ the nonrelativistic expansion of the matrix element of the electromagnetic current is

$$
\begin{aligned}
&\left\langle\mathbf{p}^{\prime}, j\left|J_{e m}^{0}\right| \mathbf{p}, i\right\rangle= e\left[F_{C}\left(q^{2}\right) \delta_{i j}+\frac{1}{2 M_{d}^{2}} F_{\mathcal{Q}}\left(q^{2}\right)\left(\mathbf{q}_{i} \mathbf{q}_{j}-\frac{1}{3} \mathbf{q}^{2} \delta_{i j}\right)\right]\left(\frac{E+E^{\prime}}{2 M_{d}}\right) \\
&\left\langle\mathbf{p}^{\prime}, j\left|\mathbf{J}_{\text {em }}^{k}\right| \mathbf{p}, i\right\rangle= \frac{e}{2 M_{d}}\left[F_{C}\left(q^{2}\right) \delta_{i j}\left(\mathbf{p}+\mathbf{p}^{\prime}\right)^{k}+F_{M}\left(q^{2}\right)\left(\delta_{j}^{k} \mathbf{q}_{i}-\delta_{i}^{k} \mathbf{q}_{j}\right)\right. \\
&\left.\quad+\frac{1}{2 M_{d}^{2}} F_{\mathcal{Q}}\left(q^{2}\right)\left(\mathbf{q}_{i} \mathbf{q}_{j}-\frac{1}{3} \mathbf{q}^{2} \delta_{i j}\right)\left(\mathbf{p}+\mathbf{p}^{\prime}\right)^{k}\right] . \quad(52)
\end{aligned}
$$

Relativistic corrections to these formulae enter at $\mathrm{N}^{2} \mathrm{LO}$. In Eq. (52) $q^{2}=$ $q_{0}^{2}-|\mathbf{q}|^{2}$, is the square of the four-momentum transfer. Momentum conservation implies $\mathbf{q}=\mathbf{p}^{\prime}-\mathbf{p}$. The dimensionless form factors defined in Eq. (52) are normalized such that

$$
F_{C}(0)=1, \quad \frac{e}{2 M_{d}} F_{M}(0)=\mu_{M}, \quad \frac{1}{M_{d}^{2}} F_{\mathcal{Q}}(0)=\mu_{\mathcal{Q}},
$$

where $\mu_{M}=0.85741 \frac{e}{2 M}$ is the deutergn magnetic moment, and $\mu_{\mathcal{Q}}=0.2859 \mathrm{fm}^{2}$ is the deuteron quadrupole moment 45,46 . 
As an example, the calculation of the electric-quadrupole form-factor of the deuteron will be outlined. In EFT( $\Uparrow$ ) the physics of this form factor at LO is exactly the same as in ERT. The photon couples to the D-state component of the deuteron wave function, which in $\operatorname{EFT}(\hbar)$ appears through a perturbative insertion of ${ }^{\pi} C_{0,-1}^{(s d)}$. Some of the NLO corrections, those that serve to give the deuteron its correct asymptotic S-state normalization, could be computed in ERT. However, there is also a contribution beyond ERT, depicted in diagram (d) of Fig. 9, where a quadrupole photon mediates an S-to-S transition in the deuteron.

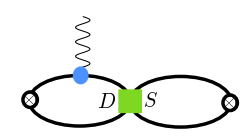

(a)

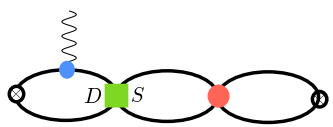

(b)

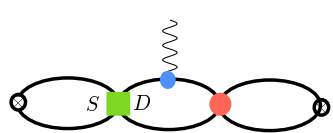

(c)

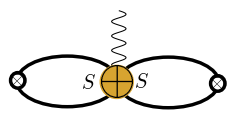

$(d)$

Figure 9: Some LO and NLO contributions to the deuteron quadrupole form factor. Diagrams of the form of (a) contribute at LO and higher. Diagrams of the form of (b) and (c) contribute at NLO and higher. At NLO there is a contribution from a local counterterm, diagram (d).

At LO, the contribution from diagram (a) in Fig. 9 is, after performing the relative-energy-integrations,

$$
\begin{aligned}
\Gamma_{\mathrm{LO}}^{(a)}= & M^{3 \pi} C_{0,-1}^{(s d)} \int \frac{d^{n-1} \mathbf{k}}{(2 \pi)^{3}} \frac{d^{n-1} \mathbf{l}}{(2 \pi)^{3}} \frac{\left(\mathbf{k}+\frac{\mathbf{q}}{2}\right)^{a}\left(\mathbf{k}+\frac{\mathbf{q}}{2}\right)^{b}-\frac{\delta^{a b}}{n-1}\left|\mathbf{k}+\frac{\mathbf{q}}{2}\right|^{2}}{\left[\left|\mathbf{l}+\frac{\mathbf{q}}{2}\right|^{2}+\gamma^{2}\right]\left[\left|\mathbf{k}+\frac{\mathbf{q}}{2}\right|^{2}+\gamma^{2}\right]\left[|\mathbf{k}|^{2}+\gamma^{2}\right]} \\
= & \frac{1}{|\mathbf{q}|^{2}}\left[\mathbf{q}^{a} \mathbf{q}^{b}-\frac{\delta^{a b}}{n-1}|\mathbf{q}|^{2}\right] M^{3 \pi} C_{0,-1}^{(s d)}\left(\frac{n-1}{n-2}\right)\left(\frac{\mu-\gamma}{4 \pi}\right) \\
& \int \frac{d^{n-1} \mathbf{k}}{(2 \pi)^{3}}\left[\frac{\frac{1}{2}-\frac{1}{n-1}}{|\mathbf{k}|^{2}+\gamma^{2}}+\frac{\frac{\gamma^{2}}{n-1}+\frac{|\mathbf{q}|^{2}}{16}}{\left[\left|\mathbf{k}+\frac{\mathbf{q}}{2}\right|^{2}+\gamma^{2}\right]\left[|\mathbf{k}|^{2}+\gamma^{2}\right]}\right] \\
= & \frac{1}{|\mathbf{q}|^{2}}\left[\mathbf{q}^{a} \mathbf{q}^{b}-\frac{\delta^{a b}}{n-1}|\mathbf{q}|^{2}\right] M^{3 \pi} C_{0,-1}^{(s d)}\left(\frac{\mu-\gamma}{4 \pi}\right)
\end{aligned}
$$




$$
\frac{3}{4 \pi}\left[-\frac{\gamma}{12}+\left(\frac{\gamma^{2}}{3}+\frac{|\mathbf{q}|^{2}}{16}\right) \frac{1}{|\mathbf{q}|} \tan ^{-1}\left(\frac{|\mathbf{q}|}{4 \gamma}\right)\right],
$$

where $\mathbf{q}$ is the three-momentum transfer to the deuteron, and $n$ is the number of space-time dimensions. The integrals appearing in Eq. (54) have been regulated with PDS. There are no $n-3$ poles in the $\mathbf{k}$ integration in Eq. (54) because the integral is convergent. Including the hermitean conjugate of diagram (a) and wavefunction renormalization the LO quadrupole form factor is

$$
\frac{1}{M_{d}^{2}} F_{\mathcal{Q}}(|\mathbf{k}|)=\frac{3 \eta_{s d} Z_{d}}{2 \sqrt{2}|\mathbf{k}|^{3}}\left[-4 \gamma|\mathbf{k}|+\left(16 \gamma^{2}+3|\mathbf{k}|^{2}\right) \tan ^{-1}\left(\frac{|\mathbf{k}|}{4 \gamma}\right)\right] .
$$

At NLO, there are contributions from all of the diagrams in Fig. 9. They are straightfoward to compute, and we will not present the calculation here. The contribution from a four-nucleon-one-quadrupole-photon operator 59 , diagram (d) in Fig. 9, has a coefficient ${ }^{\pi} C_{\mathcal{Q}}$ defined by the Lagrange density

$$
\begin{aligned}
\mathcal{L} & =\frac{e^{\star} C_{\mathcal{Q}}}{2}\left(N^{T} P_{i} N\right)^{\dagger}\left(N^{T} P_{j} N\right)\left[\nabla^{i} \mathbf{E}^{j}+\nabla^{j} \mathbf{E}^{i}-\frac{2}{n-1} \delta^{i j} \nabla \cdot \mathbf{E}\right] \\
& =-e^{\star} C_{\mathcal{Q}}\left(N^{T} P_{i} N\right)^{\dagger}\left(N^{T} P_{j} N\right)\left(\nabla^{i} \nabla^{j}-\frac{1}{n-1} \nabla^{2} \delta^{i j}\right) A^{0}+\ldots .,(56)
\end{aligned}
$$

where $\mathbf{E}$ is the electric field operator.

At $\mathrm{N}^{2} \mathrm{LO}$ the only correction comes from a modification to the one-body charge operator. This modification introduces the finite charge-radius of the nucleon into the calculation. Thus, the deuteron quadrupole form factor in $\operatorname{EFT}(\Uparrow)$ is, up to $\mathrm{N}^{2} \mathrm{LO}$ :

$$
\begin{array}{ll}
\frac{1}{M_{d}^{2}} & F_{\mathcal{Q}}(|\mathbf{q}|)=\delta \mu_{\mathcal{Q}} \\
+ & \frac{3 Z_{d} \eta_{s d}}{2 \sqrt{2} \gamma|\mathbf{q}|^{3}}\left[-4|\mathbf{q}|\left(\gamma+\frac{1}{6}|\mathbf{q}|^{2} \frac{Z_{d}-1}{\gamma Z_{d}}\right)+\left(3|\mathbf{q}|^{2}+16 \gamma^{2}\right) \tan ^{-1}\left(\frac{|\mathbf{q}|}{4 \gamma}\right)\right] \\
+ & \frac{\eta_{s d}\left\langle r_{N, 0}^{2}\right\rangle}{4 \sqrt{2} \gamma|\mathbf{q}|}\left[4 \gamma|\mathbf{q}|+\left(3|\mathbf{q}|^{2}+16 \gamma^{2}\right) \tan ^{-1}\left(\frac{|\mathbf{q}|}{4 \gamma}\right)\right]
\end{array}
$$

where $r_{N, 0}$ is the isoscalar nucleon charge radius. Note that we have eliminated the bare coupling ${ }^{\pi} C_{\mathcal{Q}}$ in favor of the RG-invariant quantity $\delta \mu_{\mathcal{Q}}$. The value of $\delta \mu_{\mathcal{Q}}$ is chosen so as to reproduce the observed deuteron quadrupole moment, $\mu_{Q}=F_{\mathcal{Q}}(0) / M_{d}^{2}=\delta \mu_{\mathcal{Q}}+\frac{\eta_{s d}}{\sqrt{2} \gamma^{2}}$. The value that does this is $\delta \mu_{\mathcal{Q}}=$ $-0.0492 \mathrm{fm}^{2}$. 


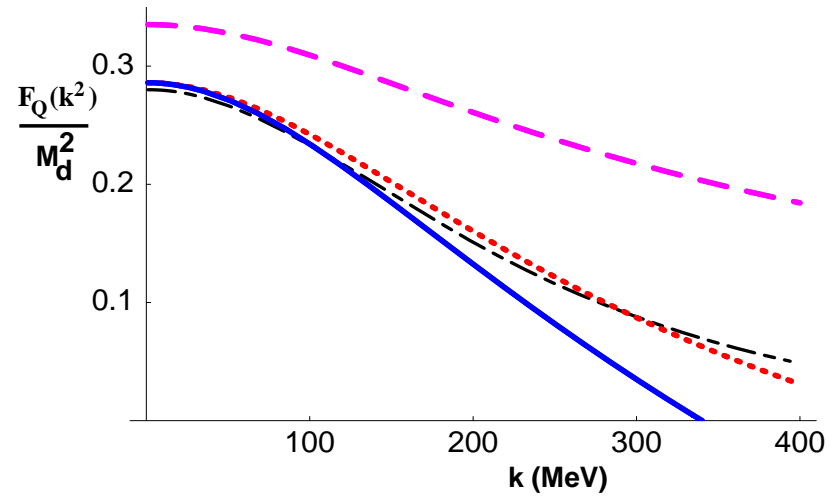

Figure 10: The deuteron quadrupole form factor. The dashed, dotted and solid curves correspond to the $\mathrm{LO}, \mathrm{NLO}$ and $\mathrm{N}^{2} \mathrm{LO}$ predictions. The dot-dashed curve eepresponds to a calculation with the Bonn-B potential in the formulation of 4 .

Once the quadrupole moment is fixed we have a prediction for the $q^{2}$ dependence of $F_{Q}$. The form factor computed at $\mathrm{LO}, \mathrm{NLO}$ and $\mathrm{N}^{2} \mathrm{LO}$ is shown in Fig. 10, along with a potential-modelcalculation performed with the Bonn-B potential in the formulation of Ref. 47 . The second and third terms in Eq. (57) would be reproduced in ERT, as long as ${ }^{3} S_{1}-{ }^{3} D_{1}$ mixing and the contribution from the finite size of the nucleon were included in ERT. The first term in Eq. (57) is a contribution beyond ERT, and corresponds to an insertion of the lowest deuteron quadrupole counterterm not constrained by $N N$ scattering alone. The presence of this counterterm at NLO indicates that $\mu_{\mathcal{Q}}$ is sensitive to short-distance physics - a fact confirmed by results gleaned from potential-model calculations, and calculations in EFTs with pions (see below). The absence of the appropriate short-distance physics in potential model calculations is presumablyt the reason that the best of these calculations underpredict $\mu_{\mathcal{Q}}$ by about $5 \%$ in.

In contrast, we can define the deuteron's quadrupole "radius", via:

$$
\left\langle r_{Q}^{2}\right\rangle=-\frac{1}{6 M_{d}^{2}} \frac{d}{d|\mathbf{q}|^{2}} F_{Q}(|\mathbf{q}|) .
$$

At $\mathrm{N}^{2} \mathrm{LO}$ this quantity is given by:

$$
\left\langle r_{Q}^{2}\right\rangle=\frac{9 \eta_{s d}}{80 \sqrt{2} \gamma^{4}}\left(Z_{d}+\frac{80}{9}\left\langle r_{N, 0}^{2}\right\rangle \gamma^{2}\right)=1.398 \mathrm{fm}^{4},
$$

independent of the quadrupole counterterm. $\left\langle r_{Q}^{2}\right\rangle$ is, not surprisingly, less sen- 
sitive to short-distance physics than $\mu_{\mathcal{Q}}$. Therefore, we expect that potentialmodel calculations of this quantity will agree substantially better with observation and with each other, than potential-model calculations of the quadrupole moment will.

Similar calculations can be performed for the charge and the magnetic form factors. At $\mathrm{LO}, \mathrm{NLO}$ and $\mathrm{N}^{2} \mathrm{LO}$ the contributions to the charge form factor are

$$
\begin{aligned}
& F_{C}^{(0)}(|\mathbf{q}|)=\frac{4 \gamma}{|\mathbf{q}|} \tan ^{-1}\left(\frac{|\mathbf{q}|}{4 \gamma}\right), F_{C}^{(1)}(|\mathbf{q}|)=\left(Z_{d}-1\right)\left(F_{C}^{(0)}(|\mathbf{q}|)-1\right) \\
& F_{C}^{(2)}(|\mathbf{q}|)=-\frac{1}{6}\left\langle r_{N, 0}^{2}\right\rangle|\mathbf{q}|^{2} F_{C}^{(0)}(|\mathbf{q}|) .
\end{aligned}
$$

The sum of the LO, NLO and $\mathrm{N}^{2} \mathrm{LO}$ contributions is exactly what one obtains in ERT. At the next order, $\mathrm{N}^{3} \mathrm{LO}$, there is a contribution from a four-nucleonone-photon interaction that represents physics beyond ERT. The deuteron charge radius that arises from the sum of the form factors in Eq. (60) is, including the leading relativistic effect 39 ,

$$
\left\langle r_{d}^{2}\right\rangle^{\mathrm{EFT}}=\left\langle r_{N, 0}^{2}\right\rangle+\frac{Z_{d}}{8 \gamma^{2}}+\frac{1}{32 M^{2}}=4.565 \mathrm{fm}^{2},
$$

which is to be compared with the experimental value $\left\langle r_{d}^{2}\right\rangle=4.538 \mathrm{fm}^{2} 48,490.51$. The $\operatorname{EFT}(t)$ calculation agrees with data (and with potential-model calculations too) to within $\sim \frac{1}{2} \%$.

The magnetic form factor of the deuteron is dominated by contributions from the single nucleon magnetic moments. The Lagrange density describing the magnetic interactions of the nucleons is

$$
\mathcal{L}_{1, B}=\frac{e}{2 M} N^{\dagger}\left(\kappa_{0}+\kappa_{1} \tau_{3}\right) \sigma \cdot \mathbf{B} N
$$

where $\kappa_{0}=\frac{1}{2}\left(\kappa_{p}+\kappa_{n}\right)$ and $\kappa_{1}=\frac{1}{2}\left(\kappa_{p}-\kappa_{n}\right)$ are the isoscalar and isovector nucleon magnetic moments in nuclear magnetons, with $\kappa_{p}=2.79285 \mathrm{NM}$ and $\kappa_{n}=-1.91304 \mathrm{NM}$. The magnetic field is conventionally defined $\mathbf{B}=\nabla \times \mathbf{A}$. The magnetic form factor of the deuteron at LO and NLO is

$$
\begin{aligned}
& F_{M}^{(0)}\left(|\mathbf{q}|^{2}\right)=2 \kappa_{0} \frac{4 \gamma}{|\mathbf{q}|} \tan ^{-1}\left(\frac{|\mathbf{q}|}{4 \gamma}\right) \\
& F_{M}^{(1)}\left(|\mathbf{q}|^{2}\right)=\left(Z_{d}-1\right) F_{M}^{(0)}\left(|\mathbf{q}|^{2}\right)-2 \kappa_{0}\left(Z_{d}-1\right)+L_{2} \frac{\gamma(\mu-\gamma)}{\pi} .
\end{aligned}
$$




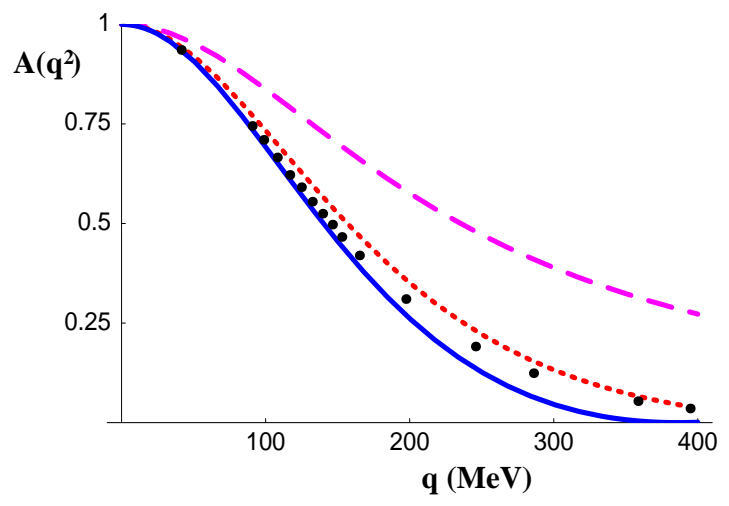

Figure 11: The form factor $A\left(q^{2}\right)$ (dominated by the square of the charge form factor) measured in electron-deuteron scattering. The dashed, dotted and solid curves correspond to the LO, NLO and $\mathrm{N}^{2} \mathrm{LO}$ form factors. The solid circles correspond to data.

Note that at LO the result is just the charge form factor multiplied by the isoscalar anomalous magnetic moment. The first piece of the NLO result has a similar interpretation, but another piece also appears at this order. This second contribution represents the effect of a two-body current on $F_{M}$. The coefficient, $L_{2}$, which appears there is the coefficient of a four-nucleon-onemagnetic-photon term in the Lagrange density 39.52

$$
\mathcal{L}=-e^{\sharp t} L_{2} i \epsilon_{i j k}\left(N^{T} P_{i} N\right)^{\dagger}\left(N^{T} P_{j} N\right) \mathbf{B}_{k}+\text { h.c. }
$$

The value of this coefficient can be found by demanding that the the deuteron magnetic moment is reproduced at NLO. Since the NLO result for $\mu_{M}$ in the Q-expansion is 22

$$
\mu_{M}=\frac{e}{2 M}\left(\kappa_{p}+\kappa_{n}+{ }^{\pi} L_{2} \frac{2 M \gamma(\mu-\gamma)^{2}}{\pi}\right),
$$

we reguire that if $L_{2}$ is evaluated at the scale $\mu=m_{\pi}$ then it must have the value ${ }^{9} .52{ }^{t} L_{2}\left(m_{\pi}\right)=-0.149 \mathrm{fm}^{4}$. This is significantly smaller than the naively estimated size of $\sim 1 \mathrm{fm}^{4}$.

\subsection{Deuteron Polarizabilities}

The electromagnetic polarizabilities of a hadron measure its deformation in external electric and magnetic fields (for an overview see 53 ). The electric 
polarizability of the deptergp $\alpha_{E 0}$, has been investigated thoroughly with $N N$ potential models 54 55,56,57. To a very high precision one obtains $\alpha_{E 0}=$ $0.6328 \pm 0.0017 \mathrm{fm}^{3}$ with those techniques. This is not surprising since the electric polarizability is dominated by the long-range behavior of the deuteron wavefunction. Thus, if a model is tuned to reproduce this asymptotic part of the deuteron wavefunction then the predicted electric polarizability should be very close to nature.

Similarly in $\operatorname{EFT}(\hbar) \alpha_{E 0}$ can be calculated to high precision. The contributions from the first four orders in perturbation theory give an electric polarizability of the deuteron which is (including a relativistic correction)

$$
\alpha_{E 0}^{(z)}=\frac{\alpha M}{32 \gamma^{4}}\left[1+\left(Z_{d}-1\right)+\frac{2 \gamma^{2}}{3 M^{2}}+\frac{M \gamma^{3}}{3 \pi} D_{P}\right]
$$

where $D_{P}={ }^{\star} C_{2}^{\left({ }^{3} P_{0}\right)}+2{ }^{\star} C_{2}^{\left({ }^{3} P_{1}\right)}+\frac{20}{3}{ }^{\pi} C_{2}^{\left({ }^{3} P_{2}\right)}=-1.51 \mathrm{fm}^{3}$ is a combination of $\mathrm{P}$-wave coefficients. The same combination of $\mathrm{P}$-wave scattering parameters contributes to the $\mathrm{E} 1$ amplitude in $n p \rightarrow d \gamma$. Higher contributions are estimated to be $\lesssim 1 \%$.

In fact, in general, insertions of higher-dimension operators will also pick up factors of $Z_{d}$ when computed to higher orders and therefore we provocatively write

$$
\begin{aligned}
\alpha_{E 0}^{(z)} & =\frac{\alpha M}{32 \gamma^{4}} Z_{d}\left[1+\frac{2 \gamma^{2}}{3 M^{2}}+\frac{M \gamma^{3}}{3 \pi} D_{P}+\ldots\right] \\
& =0.6325 \mathrm{fm}^{3}
\end{aligned}
$$

The ellipses denote higher-order terms, which include the $\mathrm{N}^{4} \mathrm{LO}$ contribution from ${ }^{3} S_{1}-{ }^{3} D_{1}$ mixing proportional to $\eta_{s d}^{2} \sim 6.5 \times 10^{-4}$. We estimate that the uncertainty in the result shown in Eq. (67) is $\pm 0.002 \mathrm{fm}^{3}$, which includes the effect of this S-D mixing. The first contribution from interactions that are not constrained by $N N$ phase shifts or gauge invariance occurs at $\mathrm{N}^{5} \mathrm{LO}$, two orders higher than we have calculated, and hence contributes an uncertainty of $\pm 0.0007 \mathrm{fm}^{3}$.

\section{$3.3 n p \rightarrow d \gamma$}

The capture of low-energy neutrons by protons to form a deuteron is a reaction which is not only of fundamental interest in nuclear physics, but also represents a critical input to astrophysical simulations. At low energies the total crosssection is dominated by M1-isovector capture from $n p$ continuum states to the deuteron. This is the energy region relevant for Big-Bang-Nucleosynthesis 
$(\mathrm{BBN})$, and these isovector amplitudes have been computed to very high orders in EFT 58,41. Meanwhile, the much smaller isoscalar amplitudes which contribute to this transition have been computed to NLO in order to make predictions that are to be compared with imminent experimental measurements 59 .

At low energies it is useful to make a multipole decomposition for the amplitude $n p \rightarrow d \gamma$

$$
\begin{aligned}
& T=i e X_{M 1_{V}} \varepsilon^{a b c} \epsilon_{(d)}^{* a} \mathbf{k}^{b} \epsilon_{(\gamma)}^{* c} U_{\mathrm{n}}^{T} \bar{P}^{3} U_{\mathrm{p}} \\
& +\quad e X_{E 1_{V}} U_{\mathrm{n}}^{T} \tau_{2} \tau_{3} \sigma_{2} \sigma \cdot \epsilon_{(d)}^{*} U_{\mathrm{p}} \mathbf{P} \cdot \epsilon_{(\gamma)}^{*} \\
& +\quad \frac{e X_{M 1_{S}}}{\sqrt{2}} U_{\mathrm{n}}^{T} P^{a}\left[\mathbf{k}^{a} \epsilon_{(d)}^{*} \cdot \epsilon_{(\gamma)}^{*}-\epsilon_{(d)}^{*} \cdot \mathbf{k} \epsilon_{(\gamma)}^{* a}\right] U_{\mathrm{p}} \\
& +\quad \frac{e X_{E 2_{S}}}{\sqrt{2}} U_{\mathrm{n}}^{T} P^{a}\left[\mathbf{k}^{a} \epsilon_{(d)}^{*} \cdot \epsilon_{(\gamma)}^{*}+\epsilon_{(d)}^{*} \cdot \mathbf{k} \epsilon_{(\gamma)}^{* a}-\frac{2}{n-1} \epsilon_{(d)}^{* a} \mathbf{k} \cdot \epsilon_{(\gamma)}^{*}\right] U_{\mathrm{p}} .
\end{aligned}
$$

Only the lowest partial waves are shown, corresponding to electric dipole capture of nucleons in a $\mathrm{P}$-wave with amplitude $X_{E 1_{V}}$, isovector magnetic capture of nucleons in the ${ }^{1} S_{0}$ channel with amplitude $X_{M 1_{V}}$, isoscalar magnetic capture of nucleons in the ${ }^{3} S_{1}$ channel with amplitude $X_{M 1_{S}}$, and isoscalar electric quadrupole capture of nucleons in the ${ }^{3} S_{1}$ channel with amplitude $X_{E 2_{S}}$. Higher multipoles are suppressed by additional powers of $\mathbf{k}$, the photon momentum. Note that $U_{\mathrm{n}}$ is the neutron Pauli spinor and $U_{\mathrm{p}}$ is the proton Pauli spinor, while $|\mathbf{p}|$ is the magnitude of the momentum of each nucleon in the $N N$ center-of-mass frame. The photon polarization vector is $\epsilon_{(\gamma)}$, and $\epsilon_{(d)}$ is the deuteron polarization vector. For convenience, we define dimensionless variables $\tilde{X}$, as follows:

$$
\frac{|\mathbf{P}| M_{N}}{\gamma^{2}+|\mathbf{P}|^{2}} X_{E 1_{V}}=i \frac{2}{M} \sqrt{\frac{\pi}{\gamma^{3}}} \tilde{X}_{E 1_{V}} \quad, \quad X_{\pi L_{I}}=i \frac{2}{M} \sqrt{\frac{\pi}{\gamma^{3}}} \tilde{X}_{\pi L_{I}}
$$

where $X_{\pi L_{I}}$ are the amplitudes other than $X_{E 1_{V}}$. In terms of the amplitudes given in Eq. (68) and Eq. (69) the unpolarized cross-section is

$$
\sigma=\frac{8 \pi \alpha \gamma^{3}}{M_{N}^{5}|\mathbf{v}|}\left[\left|\tilde{X}_{M 1_{V}}\right|^{2}+\left|\tilde{X}_{E 1_{V}}\right|^{2}+\left|\tilde{X}_{M 1_{S}}\right|^{2}+\left|\tilde{X}_{E 2_{S}}\right|^{2}\right]
$$

where $\alpha$ is the fine-structure constant, and $|\mathbf{v}|$ is the velocity of the incoming neutrons. Close to threshold $\tilde{X}_{M 1_{V}}$ is the dominant contribution to this cross section, being larger than the other multipoles by several orders of magnitude. Therefore a measurement of $\sigma$ in this kinematic regime does not constrain $\tilde{X}_{E 1_{V}}, \tilde{X}_{M 1_{S}}$, or $\tilde{X}_{E 2_{S}}$. 
The isovector $M 1_{V}$ amplitude up to NLO is

$$
\begin{aligned}
\left|\tilde{X}_{M 1}\right|^{2}= & \frac{\kappa_{1}^{2} \gamma^{4}\left(\frac{1}{a_{1}}-\gamma\right)^{2}}{\left(\frac{1}{a_{1}^{2}}+|\mathbf{P}|^{2}\right)\left(\gamma^{2}+|\mathbf{P}|^{2}\right)^{2}} \\
& {\left[Z_{d}-r_{0} \frac{\left(\frac{\gamma}{a_{1}}+|\mathbf{P}|^{2}\right)|\mathbf{P}|^{2}}{\left(\frac{1}{a_{1}^{2}}+|\mathbf{P}|^{2}\right)\left(\frac{1}{a_{1}}-\gamma\right)}-\frac{L_{n p}}{\kappa_{1}} \frac{M}{2 \pi} \frac{\gamma^{2}+|\mathbf{P}|^{2}}{\frac{1}{a_{1}}-\gamma}\right], }
\end{aligned}
$$

where the first term can be obtained in ERT 60. However, ERT cannot reproduce the cross-section for this process, giving $10 \%$ less than the experimental result for the thermal neutron capture cross-section 61 for incident neutrons with $|v|=2200 \mathrm{~m} / \mathrm{s}$ of $\sigma_{\mathrm{th}}=(334.2 \pm 0.5) \mathrm{mb}$. In the traditional picture this $10 \%$ discrepancy was explained by Riska and Brown 62 as being due to twobody currents. These mechanisms contribute significantly to the cross-section for thermal neutron capture at threshold. Below we will discuss work by Park, Min, and Rho, which shows that this explanation emerges naturally in the EFT with pions 6364 .

In contrast, in $\operatorname{EFT}(\hbar)$ the pion is regarded as "short-range" dynamics and this two-body current appears as the isovector contact term $L_{n p}$. The constant $L_{n p}$ is an RG-invariant combination of parameters. $L_{n p}$ is related to the couplings that appear in the Lagrangian via

$$
L_{n p}=(\mu-\gamma)\left(\mu-\frac{1}{a_{1}}\right)\left[{ }^{\star} L_{1}-\frac{\pi \kappa_{1}}{M_{N}}\left(\frac{r_{0}^{\left({ }^{1} S_{0}\right)}}{\left(\mu-\frac{1}{a^{\left(1 S_{0}\right)}}\right)^{2}}+\frac{\rho_{d}}{(\mu-\gamma)^{2}}\right)\right],
$$

where the Lagrange density describing four-nucleon-one-magnetic-photon interaction is

$$
\mathcal{L}=e^{\not t} L_{1}\left(N^{T} P_{i} N\right)^{\dagger}\left(N^{T} \bar{P}^{a} N\right) \delta^{a 3} \mathbf{B}_{i}+\text { h.c. } .
$$

The coefficient of this operator, which involves four nucleon fields and a magnetic photon, is not related to $N N$ scattering data by gauge invariance. Hence it must be fitted to data on the interactions of the magnetic photon with the two-nucleon system. The value for $L_{n p}$ is determined by the requirement that we reproduce the cross-section $\sigma^{\text {th }}$.

Once the value for the $M 1_{V}$ amplitude is fixed at threshold its energydependence is determined by information from the $N N$ system. But it ceases 
to be the dominant contribution once incoming $n p$ energies of a few $\mathrm{MeV}$ are reached. The E1 amplitude becomes increasingly important as the energy of the emitted photon increases. This amplitude has been computed up to $\mathrm{N}^{3} \mathrm{LO}$, and the result is:

$$
\left|\tilde{X}_{E 1}\right|^{2}=\frac{|\mathbf{P}|^{2} M^{2} \gamma^{4}}{\left(\gamma^{2}+|\mathbf{P}|^{2}\right)^{4}}\left[Z_{d}+\frac{M \gamma}{6 \pi}\left(\frac{\gamma^{2}}{3}+|\mathbf{P}|^{2}\right) \mathcal{D}^{(P)}\right]
$$

With this result in hand, and the value for $L_{n p}$ fixed at threshold, the photodissociation cross-section for the inverse process, $\gamma d \rightarrow n p$ can be determined. The results are shown in Fig. 12.

The amplituges in Eq. (71) and Eq. (74) have been computed to one higher order by Rupal 41 . This necessitates the inclusion of relativistic effects and a counterterm for the E1 multipole. The result is then a calculation of $n p \rightarrow d \gamma$ which has an error of $\sim 1 \%$. Since the physics in these EFT calculations is very similar to the physics of potential-model calculations of this process it would seem that these "traditional" approaches can also calculate this reaction with comparable precision if they reproduce $\sigma^{\text {th }}$ accurately 65 . Recent calculations confirm that this is indeed the case.

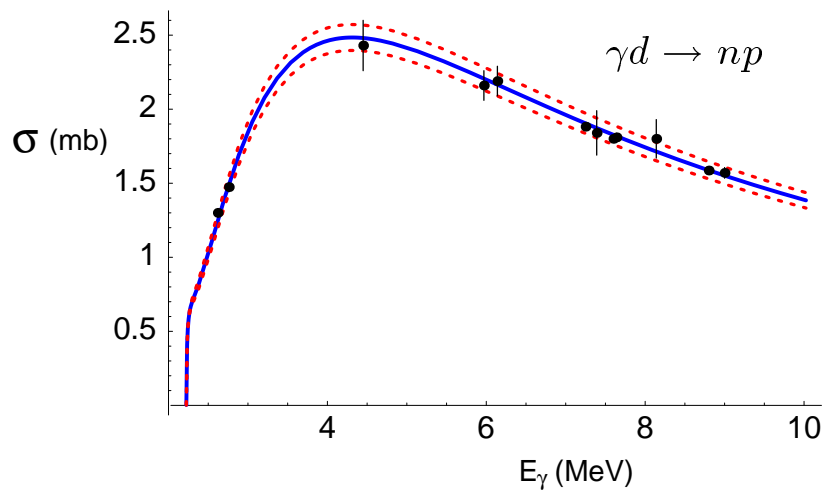

Figure 12: The photodissociation cross-section for $\gamma d \rightarrow \nexists q \rightarrow^{\text {The solid line results from }}$ Eq. (70) evaluated with the amplitudes Eq. (71) and Eq. (74)58. $L_{n p}$ is determined by the cross-section for $n p \rightarrow \gamma \gamma, \sigma^{\text {th }}$. The dashed lines denote the $\sim 3 \%$ theoretical unfertainty estimated in Ref58. Rupak has further reduced this uncertainty to below $1 \% 41$.

We now turn out attention to polarized neutron capture. For a neutron of polarization $\eta_{\mathrm{n}}$ incident upon an unpolarized proton target, the cross-sections for the production of right-handed and left-handed circularly-polarized photons are different. Defining the asymmetry $A_{\eta_{\mathrm{n}}}^{\gamma}(\theta)$ to be the ratio of the difference 
and sum of these two cross-sections, we can express this observable in terms of the amplitudes $\tilde{X}_{\pi L_{I}}$,

$$
A_{\eta_{\mathrm{n}}}^{\gamma}(\theta)=\eta_{\mathrm{n}}\left[\left(P_{\gamma}(M 1)+P_{\gamma}(E 2)\right) \cos \theta+P_{\gamma}(E 1) \sin ^{2} \theta\right],
$$

where

$$
P_{\gamma}\left(\pi L_{S}\right)=\frac{\sqrt{2} \operatorname{Re}\left[\tilde{X}_{M 1_{V}} \tilde{X}_{\pi L_{S}}^{*}\right]}{\left|\tilde{X}_{M 1_{V}}\right|^{2}}, P_{\gamma}(E 1)=\frac{\operatorname{Re}\left[\tilde{X}_{M 1_{V}} \tilde{X}_{E 1_{V}}^{*}\right]}{\left|\tilde{X}_{M 1_{V}}\right|^{2}} .
$$

Where we have neglected the small $M 1_{S}, E 2_{S}$ and $E 1_{V}$ amplitudes in the denominators.

The isoscalar E2 amplitude has been computed to $\mathrm{N}^{2} \mathrm{LO}$ in Ref. 40 ,

$$
\tilde{X}_{E 2_{s}}=\frac{\delta \mu_{\mathcal{Q}} \gamma^{2}}{4 \sqrt{2}}-\frac{\eta_{s d}}{10}\left(1+\frac{3}{8}\left(Z_{d}-1\right)-\frac{7}{32}\left(Z_{d}-1\right)^{2}\right)+\frac{3 \gamma^{4}}{40} E_{1}^{(4)}
$$

The deuteron quadrupole moment counterterm appears here, and once it is fixed there are no free parameters in this expression. Since $\tilde{X}_{M 1_{V}}$ was computed to $\mathrm{N}^{2} \mathrm{LO}$, this leads to a series for the ratio of isoscalar E2 to isovector M1 matrix elements which has the numerical values

$$
\frac{\tilde{X}_{E 2_{S}}}{\tilde{X}_{M 1_{V}}}=-[1.565+0.693+0.209] \times 10^{-4}=-2.47 \times 10^{-4} .
$$

Note that this series appears to be converging well, with an expansion parameter of about one third.

The convergence of the isoscalar M1 amplitude is harder to assess. At NLO this amplitude is:

$$
\tilde{X}_{M 1_{S}}^{(1)}=\sqrt{2} \frac{M_{N} \gamma}{2 \pi}{ }^{\pi} L_{2}(\mu-\gamma)^{2} .
$$

Once again a counterterm that was fitted to a deuteron static property - in this case the magnetic moment - makes an appearance. While this is formally the leading contribution, the smallness of ${ }^{d} L_{2}$ suggests that the contribution given in Eq. (79) might not dominate over higher order terms, and the $M 1_{S}$ amplitude might not be predicted well by $\operatorname{EFT}(\hbar)$ at this order. Numerically,

$$
\frac{\tilde{X}_{M 1_{S}}}{\tilde{X}_{M 1_{V}}}=-5.0 \times 10^{-4}
$$

with an uncertainty that we naively estimate to be of $\sim 60 \%$. 
Using these amplitudes to compute the photon polarizations $P_{\gamma}$, we find

$$
P_{\gamma}(M 1)=-7.1 \times 10^{-4}, \quad P_{\gamma}(E 2)=-3.5 \times 10^{-4},
$$

giving a total of $P_{\gamma}=-1.06 \times 10^{-3}$ in the forward direction. This is approximately $2 / 3$ of the experimentally determined value of $66 P_{\gamma}^{\text {expt }}=-(1.5 \pm 0.3) \times$ $10^{-3}$. Given the large uncertainty in the calculation of the $M 1_{S}$ amplitude, and the uncertainty of the measurement, the two are consistent at this order. The value 39 of $P_{\gamma}(M 1)=-71 \times 10^{-4}$ agrees with the result obtained using a Reid soft-core potential 6 . However, it is significantly smaller than the ERT value of $P_{\gamma}(M 1)=-9.2 \times 10^{-4}$. However, given the large uncertainty in the $M 1_{S}$ amplitude of $\operatorname{EFT}(\not)$ these three values are consistent. They are also consistent with the calculation of Park et al. 68 . This calculation will be discussed below, since it is a calculation in an EFT with explicit pions which uses the counting of Weinberg 6 . The $\operatorname{EFT}(\hbar)$ value of $P_{\gamma}(E 2)$ quoted in Eq. (81) agrees well with that found in Ref.68. Note that the zero-range value of $P_{\gamma}(E 2): P_{\gamma}(E 2)=-2.4 \times 10^{-4}$, is reproduced at $\mathrm{LO}$ in $\mathrm{EFT}(\not)$, but it is modified by the presence of the deuteron quadrupole-moment counterterm. Hence, the $\operatorname{EFT}(\hbar)$ value of $P_{\gamma}(E 2)$ ends up lying somewhere between this ERT result, and the result obtained in a Reid soft-core calculation 67: $P_{\gamma}(E 2)=-3.7 \times 10^{-4}$.

\subsection{Neutrino-Deuteron Interactions}

Weak interaction processes involving the deuteron are central to current research efforts in nuclear physics. In addition to the accelerator based programs that will elucidate the flavor structure of the nucleon, such as the SAMPLE experiments at Bates, the interactions between neutrinos and the deuteron form the core of our efforts to learn about the neutrino and look beyond the standard model of electroweak interactions. Both in the production process, e.g. $p p \rightarrow d e^{+} \nu_{e}$, and in detection at the Sudbury Neutrino Observatory (SNO), e.g. $\nu d \rightarrow \nu n p$, charged and neutral current weak interaction matrix elements between the deuteron and continuum states are required.

Over the past few decades much effort has gone into calculating the production mechanism $p p \rightarrow d e^{+} \nu_{e}$, both from standard non-relativistic quantum mechanic 69,60 , and from sophisticated potential model technique $\$ 1$. Over time, there have been a series of progressively more sophisticated calculations of neutrino-deuteron scattering, lately with the goal of providing SNO with high-precision theoretical predictions of the cross-sections in various channels 27.83,54.76 The most sophisticated calculations, by Ying, Haxton and Henley (YHH) 78 and by Kubodera and Nosawa (KN) 5. 66 , including the 
effects of meson exchange currents (MEC's), agree with one another to within $\sim 5 \% 75,76,77,78,79$.

Recently, the presess $p p \rightarrow d e^{+} \nu_{e}$ has been examined with EFT by Kong and Ravndal 80,81,82,83 and by Park, Kubodera, Min and Rho 84. Elegant expressions and numerical values for the weak capture cross-section that are consistent with previous estimates have been obtained. The cross-section depends somewhat on the value of one four-nucleon-one-weak-gauge-boson interaction, with an energy-independent coefficient $85 L_{1, A}$, defined by the axial currents

$$
\begin{aligned}
& A_{k}^{S^{(2)}}=-2 i \varepsilon_{i j k} L_{2, A}\left(N^{T} P_{i} N\right)^{\dagger}\left(N^{T} P_{j} N\right) \\
& A_{k}^{a^{(2)}}=L_{1, A}\left(N^{T} P_{k} N\right)^{\dagger}\left(N^{T} P^{a} N\right)+\text { h.c. }
\end{aligned}
$$

and the vector currents

$$
V_{k}^{S^{(2)}}=2 i L_{2}^{s}\left(N^{T} P_{i} N\right)^{\dagger}\left(\overleftarrow{\nabla}_{i}+\vec{\nabla}_{i}\right)\left(N^{T} P_{k} N\right)+\text { h.c. }
$$

Only $L_{1, A}$ contributes at NLO as it is the only two-body operator connecting the ${ }^{1} S_{0}$ and ${ }^{3} S_{1}$ channels.
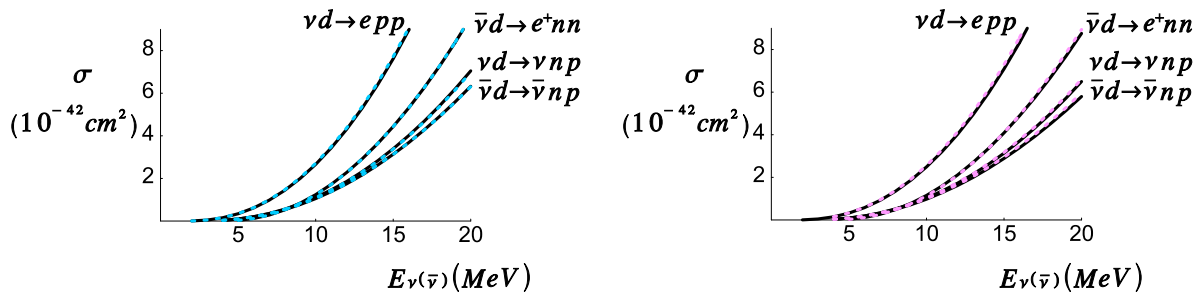

Figure 13: Inelastic $\nu(\bar{\nu}) d$ cross-sections a function of incident $\nu(\bar{\nu})$ energy. The solid curves in the left graph are from reference 79, while the dashed curves are the EFT results at $\mathrm{N}^{2} \mathrm{~m}$ fit with $L_{1, A}=5.6 \mathrm{fm}^{3}$. The solid curves in the graph are the results of YHH 78 , and the dashed curves are the $\mathrm{N}^{2} \mathrm{LO}$ EFT results 85 fit with $L_{1, A}=0.94 \mathrm{fm}^{3}$.

In both graphs, the dashed curves all lie right on top of the solid curves.

The neutrino-deuteron break-up cross-sections have been determined 8586 and it is found that $L_{1, A}$ needs to be known at the $10 \%$-level in order to perform $\mathrm{a} \sim 1 \%$ calculation of the cross-section. The YHH and KN numerical results can be recovered with different choices of $L_{1, A}$, as shown in Fig. 13, confirming that the difference between the potential-model calculations is short-distance in origin. Therefore, to predict the break-up cross-section with a precision of better than $\sim 5 \%$, one has two options. Firstly, compute the $\beta$-decay of tritium, and use this to determine the EFT counterterm $L_{1, A}$, or equivalently 
the MEC's in potential models 月. Secondly, one can perform an experiment to measure one of the break-up cross sections to high accuracy, and thereby extract $L_{1, A}$, or the MEC's. An important input into determining if neutrinos
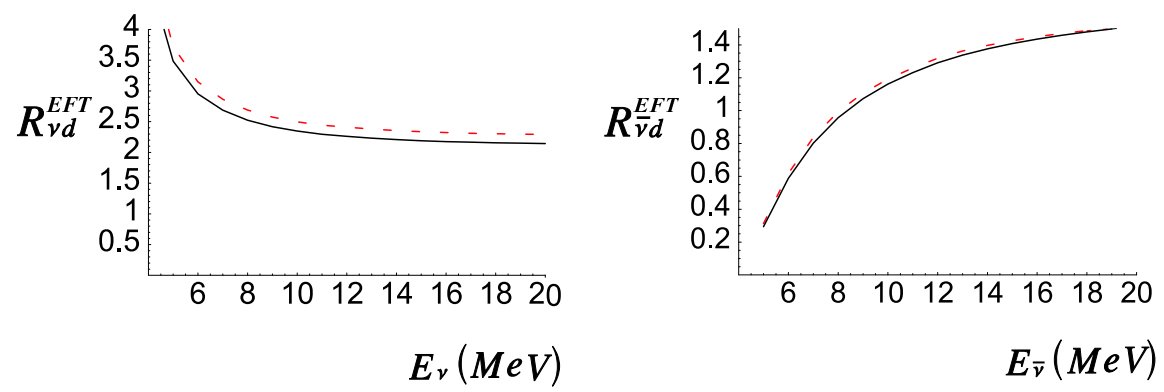

Figure 14: The ratio of charged current to neutral current cross-sections in inelastic $\nu-d$ and $\bar{\nu}-d$ scattering, as functions of incident energy. Shown are ratios of the EFT $\mathrm{N}^{2} \mathrm{LO}$ results with $L_{1, A}=-20 \mathrm{fm}^{3}$ (dashed) and $L_{1, A}=40 \mathrm{fm}^{3}$, temonstrating the insensitivity of the ratio to the value of $L_{1, A} 85$.

are changing flavor as they move through the interior of the Sun and to the Earth is the ratio of charged current to neutral current cross-sections. Fig. 14 shows that this ratio, $R_{\mathrm{EFT}}$, unlike the cross-sections themselves, is relatively insensitive to the counterterm $L_{1, A}$ (or equivalently, the MEC's).

$$
\sigma(E)=a(E)+b(E) L_{1, A}
$$

The cross-sections for two neutral current and one charged current neutrinodeuteron break-up channels are listed in Table 1, parameterized by two energy dependent functions, $a(E)$ and $b(E)$, and the energy independent counterterm $L_{1, A}$, with The magnitude of $L_{1, A}$ is estimated to be $\sim 5 \mathrm{fm}^{3}$ by NDA and is consistent with the values favored by potential models. The uncertainty introduced by omitting higher order effects is conservatively estimated to be $\sim 3 \%$ at threshgld and $\sim 2 \%$ at $20 \mathrm{MeV}$ (using $L_{1, A}=5.6 \mathrm{fm}^{3}$ the crosssections of ref. 79 are reproduced within $\sim 2 \%$ ). It can be seen from Table 1 that if $L_{1, A}$ is constrained by a $\sim 1 \%$ measurement of the $\nu_{e} d \rightarrow e^{-} p p$ crosssection, then the uncertainty in the other channels would also be $\sim 1 \%$.

$g$ With certain assumptions ahout three-body forces, this method of fixing MEC's has been implemented for $p p \rightarrow d e^{+} \nu$ ]1 


\begin{tabular}{c|ll|ll|ll} 
& \multicolumn{2}{|c|}{$\nu_{x} d \rightarrow n p \nu_{x}$} & \multicolumn{2}{c|}{$\bar{\nu}_{x} d \rightarrow n p \bar{\nu}_{x}$} & \multicolumn{2}{c}{$\nu_{e} d \rightarrow p p e^{-}$} \\
$E_{\nu, \bar{\nu}}(\mathrm{MeV})$ & $a$ & $b$ & $a$ & $b$ & $a$ & $b$ \\
\hline 4 & 0.0287 & 0.00034 & 0.0282 & 0.00034 & 0.146 & 0.0016 \\
6 & 0.188 & 0.0024 & 0.182 & 0.0024 & 0.574 & 0.0067 \\
8 & 0.514 & 0.0070 & 0.492 & 0.0069 & 1.34 & 0.017 \\
10 & 1.02 & 0.015 & 0.968 & 0.014 & 2.48 & 0.032 \\
12 & 1.72 & 0.025 & 1.61 & 0.025 & 4.01 & 0.054 \\
14 & 2.60 & 0.039 & 2.41 & 0.038 & 5.95 & 0.082 \\
16 & 3.69 & 0.056 & 3.37 & 0.054 & 8.31 & 0.12 \\
18 & 4.97 & 0.077 & 4.49 & 0.074 & 11.12 & 0.16 \\
20 & 6.47 & 0.102 & 5.76 & 0.097 & 14.39 & 0.21
\end{tabular}

Table 1: Neutrino-deuteron inelastic cross-sections from ref. 86, parameterized by $\sigma(E)=$ $a(E)+b(E) L_{1, A}$, where $a(E)$ and $b(E)$ have units of $10^{-42} \mathrm{~cm}^{2} . \nu_{X}$ denotes a standard model neutrino, $\nu_{e}, \nu_{\mu}$ or $\nu_{\tau}$.

\section{$3.5 \gamma$-Deuteron Compton Scattering}

An area of nuclear physics that is still in its infancy is $\gamma d$ Compton scattering. It is only recently that experimental measurements have been made of the differential cross-section for $\gamma d$ scattering, but this has been limited to just three energies, $E_{\gamma}=49,69$ and $95 \mathrm{MeV}$. This process is important because it may provide the only rigorous way to determine the polarizabilities of the neutron. The amplitude for $\gamma d$ Compton scattering from an unpolarized deuteron target is

$$
\mathcal{A}=-i \frac{e^{2}}{M}\left[T_{1} \varepsilon \cdot \varepsilon^{\prime}+T_{2} \mathbf{k}^{\prime} \cdot \varepsilon \mathbf{k} \cdot \varepsilon^{\prime}\right] \varepsilon_{d} \cdot \varepsilon_{d}^{\prime},
$$

where $\varepsilon$ and $\varepsilon^{\prime}$ are the polarization vectors of the initial and final state photons, and $\varepsilon_{d}$ and $\varepsilon_{d}^{\prime}$ are the polarization vectors of the initial and final state deuterons. It is straighforward to compute the contributions to both form factors, $T_{1}$ and $T_{2}$, order-by-order in the Q-expansion.

The differential cross-sections at $E_{\gamma}=49 \mathrm{MeV}$ and $69 \mathrm{MeV}$ are plotted in Fig. 15. At $E_{\gamma}=49 \mathrm{MeV}$ the theoretical expression is in very good agreement with the data at $\mathrm{N}^{3} \mathrm{LO}$. However, at $E_{\gamma}=69 \mathrm{MeV}$ there are significant differences between data and the $\mathrm{N}^{3} \mathrm{LO}$ cross-section, particularly at back angles. This is no surprise, as the theory should have failed at much lower energies. It is clear that the electric isoscalar polarizability of the nucleon, as computed at one-loop in chiral perturbation theory, makes a significant contribution to the $\gamma d$ scattering cross-section. There is good reason to believe that precise measurements of the cross-sections at low energies will allow for a good deter- 

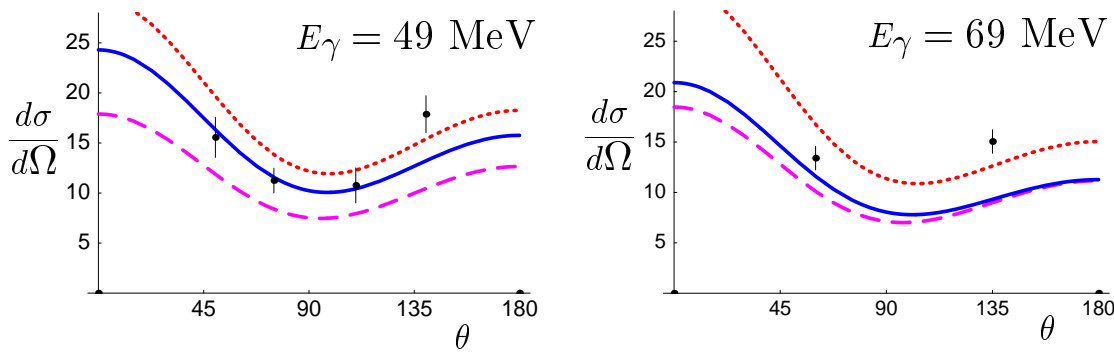

Figure 15: The differential cross-section for $\gamma d$ Compton scattering at $E_{\gamma}=49 \mathrm{MeV}$ and $69 \mathrm{MeV}$ in $\operatorname{EFT}(\not)$. The dashed curve is the LO result. The dotted curve is the $\mathrm{N}^{3} \mathrm{LO}$ result with the nucleon polarizabilities, $\alpha$ and $\beta$ set equal to zero. Fhe solid curve represents the full $\mathrm{N}^{3} \mathrm{LO}$ result. The data is from 87 .

mination of the isoscalar nucleon polarizability and hence the neutron electric polarizability.

\section{The Three-Nucleon System}

The scaling of the three-body force is the main issue to be understood in extending the results of the previous section to the three-nucleon system. Despite recent progress this is not a fully understood topic and a complete power counting at all orders does not presently exist. A number of partial results are known however. The existence of the non-trivial fixed point, and the consequent non-perturbative resummation of the two-body interactions changes the scaling of the different three-body force terms from their NDA values. In most three-body channels this change suppresses the effect of the three-body forces and makes them appear in higher orders than naively supposed. However in the $S$-wave, spin $1 / 2$ channel (where the triton and ${ }^{3} \mathrm{He}$ are) the opposite happens. The three-body force in this channel is enhanced and becomes a LO effect. The special physical property shared by no other channel is that, in this channel, Fermi statistics and/or centrifugal barrier repulsion does not block all three particles from being at the same point in space. One can then expect, on a intuitive basis, that this channel will have a larger sensitivity to short distance physics. We will see that this expectation is indeed fulfilled.

When considering three-body systems, in addition to the one- and twobody Lagrange densities in Eqs. (21) and (22) we have also three-body force 
terms

$$
\mathcal{L}_{3}=-D_{0}\left(N^{\dagger} N\right)^{3}+\cdots,
$$

where the dots represent terms involving more derivatives.

Using NDA the coefficient $D_{2 r}$ of a three-body force with $2 r$ derivatives should be of order

$$
D_{2 r} \sim \frac{4 \pi}{M \Lambda^{4+2 r}},
$$

which would imply that their effect is very suppressed compared to the twobody interactions. Close to the non-trivial fixed point however, NDA does not have to hold and $D_{2 r}$ can be enhanced by powers of $\Lambda a$ or $\Lambda / \gamma$. The natural size of $D_{2 r}(\Lambda)$ is assumed to be set by the size required to appear in the low energy expansion at the same order as the cutoff dependence of the sum of twobody diagrams. Of course, for a particular value of the cutoff $D_{2 r}(\Lambda)$ could be much smaller, but any small change in the cutoff would restore the "natural" value for $D_{2 r}(\Lambda)$. This same criterion applied to the low energy expansion around the trivial fixed point is the usual NDA argument leading to Eq. (87). The proximity of the non-trivial fixed point leads to the necessity of a nonperturbative resummation of some of the interactions. The ultraviolet behavior of an infinite sum of diagrams can be very different from the behavior of each individual diagram and this affects the scaling of the $D_{2 r}(\Lambda)$ coefficients. In all but one channel, $D_{2 r}(\Lambda)$ will be even smaller than the NDA suggests. In the $S$-wave, $J=1 / 2$ channel (where the triton and ${ }^{3} \mathrm{He}$ live) though, the opposite occurs and $D_{0}$ is a leading order effect.

\subsection{The Spin $\frac{3}{2}$ Channel}

In the spin $3 / 2$ channel 8838 the spins of all three nucleons are parallel and Fermi statistics forbids the three nucleons to occupy the same point in space. The three-body force term $D_{0}$ cannot thus contribute in this channel and only three-body terms with more derivatives might contribute. Also, only the ${ }^{3} S_{1}$ two-body interactions appear. Making the provisory assumption that the effect of the three body forces will be of higher order than our calculations we are left with a Lagrangean involving only one- and two-body terms:

$$
\begin{aligned}
\mathcal{L}_{1}+\mathcal{L}_{2} & =N^{\dagger}\left[i \partial_{0}+\frac{\vec{\nabla}^{2}}{2 M}+\cdots\right] N-{ }^{\pi} C_{0}^{\left({ }^{3} S_{1}\right)}\left(N^{T} P^{i} N\right)^{\dagger}\left(N^{T} P^{i} N\right) \\
& +\frac{{ }^{\pi} C_{2}^{\left(3 S_{1}\right)}}{8}\left[\left(N^{T} P^{i} N\right)^{\dagger}\left(N^{T} P^{a}(\vec{\nabla}-\overleftarrow{\nabla})^{2} N\right)+\text { h.c. }\right]+\cdots
\end{aligned}
$$


It is very convenient in three-body calculations to rewrite the theory described by Eq. (88) by introducing a dummy field $d^{i}$ with the quantum numbers of the deuteron (referred to hereafter as the "deuteron")

$$
\begin{aligned}
\mathcal{L}_{d} & =N^{\dagger}\left[i \partial_{0}+\frac{\vec{\nabla}^{2}}{2 M}+\cdots\right] N+d^{i \dagger}\left[-i \partial_{0}+\frac{\vec{\nabla}^{2}}{2 M}-\Delta_{d}\right] d^{i} \\
& +y_{d}\left[d^{i \dagger}\left(N^{T} P^{i} N\right)+\text { h.c. }\right]+\cdots .
\end{aligned}
$$

By performing the gaussian integration over the deuteron field and treating its kinetic term as a perturbation we recover Eq. (88) with time derivatives instead of space derivatives in the term proportional to ${ }^{\pi} C_{2}^{\left({ }^{3} S_{1}\right)}$ (and some higher order terms). This time derivative can be traded by space derivatives by use of a field redefinition

$$
N \rightarrow N+\frac{y_{d}^{2}}{8 \Delta_{d}^{2}} P^{i \dagger} N^{\dagger}\left(N^{T} P^{i} N\right)
$$

The parameters in Eq. (88) and Eq. (89) are related by

$$
y_{d}^{2}=\frac{\left({ }^{\pi} C_{0}^{\left({ }^{3} S_{1}\right)}\right)^{2}}{M^{\pi} C_{2}^{\left({ }^{3} S_{1}\right)}}, \quad \Delta_{d}=-\frac{{ }^{\pi} C_{0}^{\left({ }^{3} S_{1}\right)}}{M^{\pi} C_{2}^{\left(3 S_{1}\right)}} .
$$

With this choice of parameters Eqs. (88,89) describe equivalent theories, i.e., they both give rise to the same S-matrix. Notice that after the introduction of the deuteron field there is a large reparametrization invariance in the theory. We could for instance, change the coefficient $y_{d}$ and add an explicit two-nucleon interaction is such a way that after eliminating the deuteron field the same Lagrangean was recovered. Thus the Lagrangean in Eq. (89) is not the most general one involving nucleons and deuteron fields. The important point is that after the elimination of the deuteron field we recover the most general Lagrangean involving nucleons only. From Eq. (91) and Eq. 201 we arrive at the scaling $y_{d}^{2} \sim(4 \pi \Lambda) / M$ and $\Delta_{d} \sim(4 \pi \Lambda \mu) / M$. The scaling above shows that the kinetic term of the $d^{i}$ field is indeed sub-leading compared to the term proportional to $\Delta_{d}$.

At tree level the $d^{i}$ propagator is given by the constant $i / \Delta_{d}$. The loop corrections shown in Fig. 16 contribute to the same order as the tree level term. Summing up these graphs gives

$$
i \triangle^{i j}(p)=-\frac{4 \pi i}{M y_{d}^{2}} \frac{\delta_{d}^{i j}}{\frac{4 \pi \Delta_{d}}{M y_{d}^{2}}-\mu+\sqrt{\frac{\vec{p}^{2}}{4}-M p_{0}}} .
$$




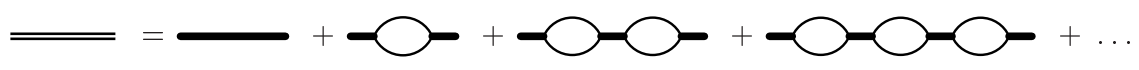

Figure 16: The leading order deuteron propagator containing the sum of bubble graphs.

The leading order $d^{i}$ field propagator is, up to a constant, the two-nucleon scattering amplitude in the ${ }^{3} S_{1}$ channel.

We consider now the scattering of a neutron on a deuteron with total spin $S=3 / 2$ (the more experimentally accessible proton-deuteron scattering would include Coulomb effects that would mask the strong interactions we are interested in here). We use the field $d^{i}$ as the interpolating field for the deuteron. The graphs that contribute to this process at leading order are depicted on the top of the Fig. 17. A simple power counting shows that they are all of the same order. The tree level diagram, for instance, contains two $d N N$ vertices $(\sim \Lambda / M)$ and one nucleon propagator $\left(\sim M / Q^{2}\right)$, for a total contribution of order $\sim \Lambda / Q^{2}$. The one loop propagator contains two additional $d N N$ vertices, two extra nucleon propagators, one $d^{i}$ field propagator $\left(\sim 1 / M y_{d}^{2} Q\right)$ and one loop $\left(\sim Q^{5} / M\right)$, thus the one loop graph is also of order $\sim \Lambda / Q^{2}$. All the graphs shown in the first line of Fig. 17 are of the same order and must be resummed at LO. This is very similar to the two-nucleon scattering case where all graphs involving only the leading interaction proportional to ${ }^{\star} C_{0}^{\left({ }^{3} S_{1}\right)}$ are leading. The difference resides on the fact that, while all the graphs for the LO two-nucleon scattering are easily summed in a geometric series, the same cannot be done in the three-body sector. The best we can do is to write an integral equation (shown in the second line of Fig. 17) whose solution, obtained numerically, equals the desired infinite sum

With the kinematic shown in Fig. 17, the $S$-wave amplitude satisfies

$$
t_{0}(k, p)=-\frac{y_{d}^{2} M}{2} K_{0}(k, p)-\frac{1}{\pi} \int_{0}^{\infty} d q q^{2} t_{0}(k, q) \frac{1}{-\gamma+\sqrt{\frac{3 q^{2}}{4}-M E}} K_{0}(q, p)
$$

\footnotetext{
${ }^{h}$ Clearly the validity of this integral equation goes beyond all orders in perturbation theory even though the derivation shown here is based on diagrams.
} 

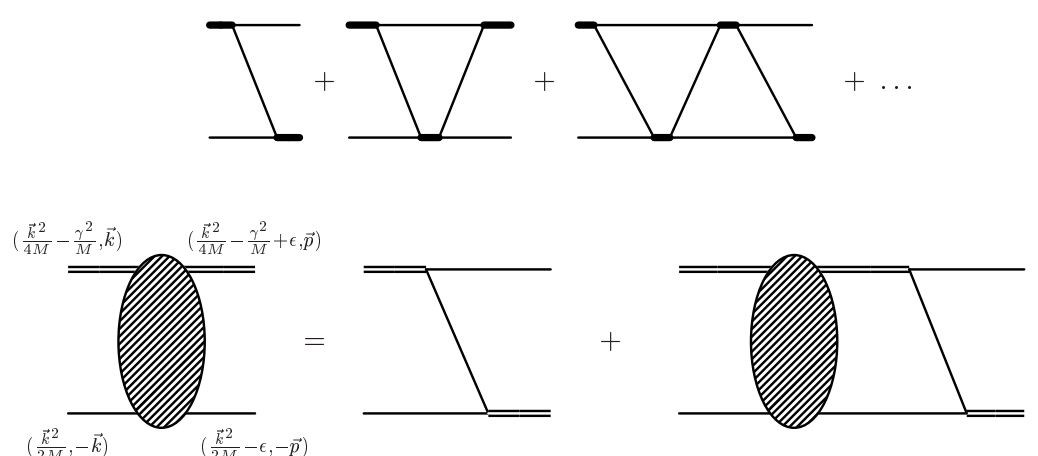

Figure 17: The first line shows the infinite sum of graphs giving the LO neutron-deuteron scattering in the spin $3 / 2$ channels. The second line is the graphical representation of the Fadeev equation that sums them up.

with

$$
K_{0}(q, p)=\frac{1}{q p} \ln \left(\frac{p^{2}+p q+q^{2}-M E}{p^{2}-p q+q^{2}-M E}\right)
$$

where $E=3 k^{2} /(4 M)-\gamma^{2} / M$ is the total energy. The projection on other partial waves lead to similar equations 89 . Notice that when $p=k$, all external legs are on-shell. This equation is just the Faddeev equation for the case of contact forces derived first in reference 90 by different methods. Eq. (93) is a one dimensional integral equation (the variable $k$ appears only as a parameter) and its numerical solution is computationally trivial. A little care must be taken with the singularities of its kernel however. Although the pole in the real axis due to the deuteron propagator is regulated by the fact that the energy should be thought as having a small positive imaginary part and the logarithmic singularity occurring above threshold is integrable, both cause numerical instabilities. An efficient way of dealing numerically with those instabilities is the Hetherington-Schick $91,92.93$ method.

To obtain the neutron-deuteron scattering amplitude we have to multiply the on-shell amplitude $t_{0}(k, p=k)$ by the wave function renormalization constant,

$$
T_{0}(k)=\sqrt{Z_{0}} t_{0}(k, k) \sqrt{Z_{0}},
$$

where

$$
\frac{1}{Z_{0}}=\left.\mathrm{i} \frac{\partial}{\partial \mathrm{p}_{0}} \frac{1}{\mathrm{i} \triangle(\mathrm{p})}\right|_{\mathrm{p}_{0}=-\frac{\gamma^{2}}{\mathrm{M}}, \overrightarrow{\mathrm{p}}=0}=\frac{\mathrm{M}^{2} \mathrm{y}_{\mathrm{d}}^{2}}{8 \pi \gamma}
$$


Unlike $t_{0}(k, p)$, the scattering amplitude $T_{0}(k)$ depends on the parameters $y_{d}$ and $\Delta_{d}$ only through the observable $\gamma=\frac{4 \pi \Delta_{d}}{M y_{d}^{2}}+\mu$.

At NLO, we have contributions coming only from insertions of the kinetic energy in the deuteron propagator. For book keeping purposes it is convenient to split the constant $\Delta_{d}$ into $\Delta_{d,-1}+\Delta_{d, 0}$ with

$$
\Delta_{d,-1}=-\frac{{ }^{\sharp} C_{0,-1}^{\left({ }^{3} S_{1}\right)}}{M^{\pi} C_{2}^{\left({ }^{3} S_{1}\right)}}, \quad \Delta_{d, 0}=-\frac{{ }^{\pi} C_{0,0}^{\left({ }^{3} S_{1}\right)}}{M^{\pi} C_{2}^{\left({ }^{3} S_{1}\right)}}=\frac{\gamma_{d}^{2}}{M} .
$$

$\Delta_{d, 0}$ is subleading compared to the leading order $\Delta_{d,-1}$ operator and is treated in perturbation theory. With this choice the position of the deuteron pole is not changed by the NLO corrections to the deuteron propagator and $\Delta_{d,-1}$ does not have to be refitted.

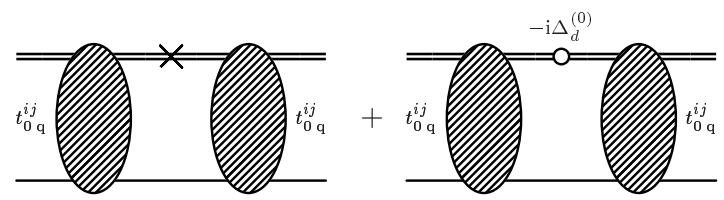

Figure 18: The NLO contributions to $n d$ scattering in the quartet channel.

The NLO corrections to neutron-deuteron scattering are shown in Fio 18. They are simply the range corrections in first order perturbation theory 249580. The graphs in Fig. 18 do not mix different partial waves and give the contribution

$$
\begin{aligned}
\mathrm{it}_{1}(\mathrm{k})=\int_{0}^{\infty} \frac{d q}{2 \pi^{2}} & q^{2}\left(\mathrm{it}_{0}(\mathrm{k}, \mathrm{q})\right)^{2}\left[\mathrm{i} \triangle\left(\frac{3 \mathrm{k}^{2}}{4 \mathrm{M}}-\frac{\gamma_{\mathrm{d}}^{2}}{\mathrm{M}}-\frac{\mathrm{q}^{2}}{2 \mathrm{M}}, \overrightarrow{\mathrm{q}}\right)\right]^{2} \\
\times & \mathrm{i} \mathcal{I}_{\mathrm{d}}\left(\frac{3 \mathrm{k}^{2}}{4 \mathrm{M}}-\frac{\gamma_{\mathrm{d}}^{2}}{\mathrm{M}}-\frac{\mathrm{q}^{2}}{2 \mathrm{M}}, \overrightarrow{\mathrm{q}}\right)
\end{aligned}
$$

where the correction to the deuteron propagator is $\mathcal{I}_{d}\left(p_{0}, \vec{p}\right)=-\Delta_{d}^{(0)}-p_{0}+\frac{\vec{p}^{2}}{4 M}$. The amplitude in Eq. (98) has to be solved for numerically as $t_{0}(k, p)$ is known only numerically. The diagrams in Fig. 18 are ultraviolet finite. The degree of convergence however is not what one might naively guess, as can be seen by 
the following argument. Each one of the diagrams in Fig. 17 behaves for large values of the external momenta $p \gg \gamma$ as $1 / p^{2}$. Their sum however has a faster decay. To see this consider Eq. (93) for large values of $p \gg k \sim \gamma$. In this case the integral is dominated by the region of integration $p \sim q$ where the kernel simplifies and we are left with 96.97

$$
t_{0}(k, p)=-\frac{2}{\sqrt{3} \pi} \int_{0}^{\infty} d q t_{0}(k, q) \frac{1}{p} \ln \left(\frac{p^{2}+p q+q^{2}}{p^{2}-p q+q^{2}}\right) .
$$

Using the ansatz $t_{0}(k, p) \sim p^{s-1}$ we find an equation that determines the power describing the decay of $t_{0}(k, p)$ for large $p$

$$
1+\frac{4}{\sqrt{3} s} \frac{\sin (\pi s / 6)}{\cos (\pi s / 2)}=0 .
$$

The negative solution (the positive ones lead to divergent integrals, cannot describe solutions of the integral equation and are discarded) is $s \simeq-2.166$. The loops in Fig. 18 converge in the ultraviolet as $\Lambda^{1+2 s} \sim \Lambda^{-3.33}$ as opposed to the naive behavior $\Lambda^{-1}$, valid at each order in a perturbation expansion in the strength of the kernel. This fact has consequences for the estimate of the three-body forces since it changes their running. As we will see below, in the triton- ${ }^{3} \mathrm{He}$ channel the opposite happens and the three-body force in that channel is enhanced compared to NDA estimates.

To complete the NLO calculation the wave function renormalization constant $Z$ is found from

$$
\frac{1}{Z}=\frac{1}{Z_{0}+Z_{1}} \simeq \frac{1}{Z_{0}}-\frac{Z_{1}}{Z_{0}^{2}}=\frac{1}{Z_{0}}-\left.\mathrm{i} \frac{\partial}{\partial \mathrm{p}_{0}} \mathrm{i} \mathcal{I}_{\mathrm{d}}\left(\mathrm{p}_{0}, \overrightarrow{\mathrm{p}}\right)\right|_{\mathrm{p}_{0}=\frac{\gamma_{\mathrm{d}}^{2}}{\mathrm{M}}, \overrightarrow{\mathrm{p}}=0}(
$$

and thus $Z_{1}=Z_{0}^{2}$. The NLO amplitude in the quartet channel is therefore given by

$$
\begin{aligned}
T(k) & =Z t(k, k) \simeq\left(Z_{0}+Z_{1}\right)\left(t_{0}(k, k)+t_{1}(k, k)\right) \\
& \simeq T_{0}(k)+Z_{0} t_{1}(k, k)+Z_{1} t_{0}(k, k)=T_{0}(k)+T_{1}(k) .
\end{aligned}
$$

The phase shift for each partial wave is extracted by expanding both sides of the relation

$$
T(k) \simeq T_{0}(k)+T_{1}(k)=\frac{3 \pi}{M} \frac{1}{k \cot \delta-i k}
$$

in $Q$ with $\delta=\delta^{(0)}+\delta^{(1)}+\ldots$ and keeping only linear terms,

$$
\delta^{(0)}=\frac{1}{2 \mathrm{i}} \ln \left(1+\frac{2 \mathrm{ik}}{3 \pi} T_{0}(k)\right), \quad \delta^{(1)}=\frac{1}{\mathrm{i}} \frac{k}{3 \pi} \frac{\left(T_{0}(k)+T_{1}(k)\right)}{\left(1+\frac{2 \mathrm{ik}}{3 \pi} T_{0}(k)\right)} .
$$


At $\mathrm{N}^{2} \mathrm{LO}$ there are two kinds of contributions. One comes from the same insertions of deuteron kinetic energy and $\Delta^{(0)}$ appearing at NLO, but now inserted twice. The other comes from inserting the LO operator describing the tensor force appearing in Eq. (105). The leading tensor force is generated by the term

$$
\mathcal{L}_{N d}^{s d}=y^{s d} d^{i \dagger} N\left((\vec{\nabla}-\overleftarrow{\nabla})^{i}(\vec{\nabla}-\overleftarrow{\nabla})^{j}-\frac{1}{3} \delta^{i j}(\vec{\nabla}-\overleftarrow{\nabla})^{2}\right) P^{j} N
$$

in the Lagrangean Eq. (89) after the gaussian integration over the deuteron fields is performed. This operators mixes spin and angular momentum and produces a splitting between the different three-body amplitudes amplitudes with the same spin $S$ and angular momentum $L$ but different values of the total angular momentum $J$. This splitting is very important in determining spin observables like the vector analyzing power $A_{y}$. However the $\mathrm{N}^{2} \mathrm{LO}$ calculation of the amplitudes is only a leading calculation of the splittings between different values of $J$ and cannot be very precise. Further, the tensor force appears only linearly and after averaging over the different values of $J$, its contribution vanishes. Here we will restrict ourselves to the averaged phase shifts and, consequently, drop the tensor force contribution at $\mathrm{N}^{2} \mathrm{LO}$. The $\mathrm{N}^{2} \mathrm{LO}$ calculation (including some higher order contributions that can be ignored) is simply performed by replacing the LO deuteron propagator in Eq. (92) by the propagator which includes all effective range effects

$$
i \triangle^{i j}(p)=-\frac{4 \pi i}{M y_{d}^{2}} \frac{\delta^{i j}}{-\gamma+\frac{1}{2} \rho_{d}\left(M p_{0}+\gamma^{2}-\frac{\vec{p}^{2}}{4}\right)+\sqrt{\frac{\vec{p}^{2}}{4}-M p_{0}}} .
$$

in the integral equation in Eq. (93).

The calculation for the spin $S=1 / 2$ channels proceeds in an analogous way to the one in the spin $3 / 2$ channels discussed above. The main difference is that two-body interactions in the spin singlet channel also contribute. We will introduce another dummy field $T^{a}$, now with the quantum numbers of two nucleons in the ${ }^{1} S_{0}$ channel

$$
\mathcal{L} \rightarrow \mathcal{L}+t^{a \dagger}\left[\left(-\mathrm{i} \partial_{0}+\frac{\vec{\partial}^{2}}{4 \mathrm{M}}\right)-\Delta_{\mathrm{t}}\right] t^{a}+y_{t}\left[t^{a \dagger}\left(N^{T} P^{a} N\right)+\text { h.c. }\right]
$$

with the constants $y_{t}$ and $\Delta_{t}$ satisfying relations analogous to Eq.91). Again, by integrating over the "dibaryon" $t^{a}$ we recover the ${ }^{1} S_{0}$ two-body interactions. 

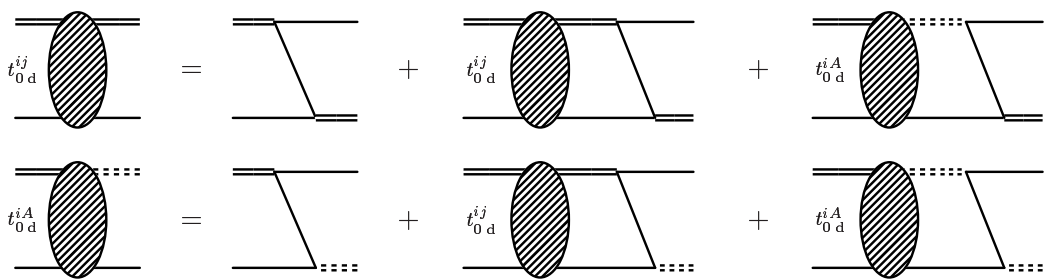

Figure 19: The coupled equations in the spin $1 / 2$ channels.

The spin zero dibaryon field $t^{a}$ contributes in intermediate states of neutrondeuteron amplitudes. The analogue of Eq. (93) is then a system of two coupled integral equations for the $d+N \rightarrow d+N\left(i t_{d}(\vec{k}, \vec{p}, \epsilon)\right)$ and $d+N \rightarrow$ $t+N\left(i t_{t}(\vec{k}, \vec{p}, \epsilon)\right)$ amplitudes.

$$
\begin{aligned}
& t_{d}(p, k)=K_{0}(p, k)+\frac{2}{\pi} \int_{0}^{\infty} \frac{q^{2} d q}{q^{2}-k^{2}} K_{0}(p, q)\left[t_{d}(q, k)+3 t_{t}(q, k)\right] \\
& t_{t}(p, k)=3 K_{0}(p, k)+\frac{2}{\pi} \int_{0}^{\infty} \frac{q^{2} d q}{q^{2}-k^{2}} K_{0}(p, q)\left[3 t_{d}(q, k)+t_{t}(q, k)\right] .
\end{aligned}
$$

The results of LO, NLO and $\mathrm{N}^{2} \mathrm{LO}$ calculations $38,88,89$ of the phase shifts for the lowest partial waves (averaged over the different values of $J$ ) are shown in Fig 20. For comparison we include the results of some old phase shift analyses 989 (that unfortunately do not contain error bars) and a recent extensive numerical calculation using modern potentials 100101 . In most cases the $\mathrm{N}^{2} \mathrm{LO}$ EFT computation seems to be convergent and accurate at the precision one would expect $\left(\left(\gamma / m_{\pi}\right)^{3} \sim 3 \%\right)$. This theoretical error is probably an overestimate because the $\mathrm{N}^{3} \mathrm{LO}$ corrections come from one insertion of the shape parameter, that is unnaturally small, three insertions of the range correction, that were already included in the $\mathrm{N}^{2} \mathrm{LO}$ calculation as discussed above and one $P$-wave insertion. There is a very precise experimental determination 102 of the quartet $S$-wave scattering length $a_{3 / 2}^{e x p}=6.35 \pm 0.02 \mathrm{fm}$. The $\mathrm{N}^{2} \mathrm{LO}$ result, $a_{3 / 2}^{E F T}=6.33 \pm 0.05 \mathrm{fm}$, compares well with this value and with calculations using second generation potential models 103.

Since the scattering lengths $a_{d}, a_{t}$, the deuteron binding momentum $1 / \gamma$ and effective range $r_{0 t}$ of the two body system in the S-waves are all input 

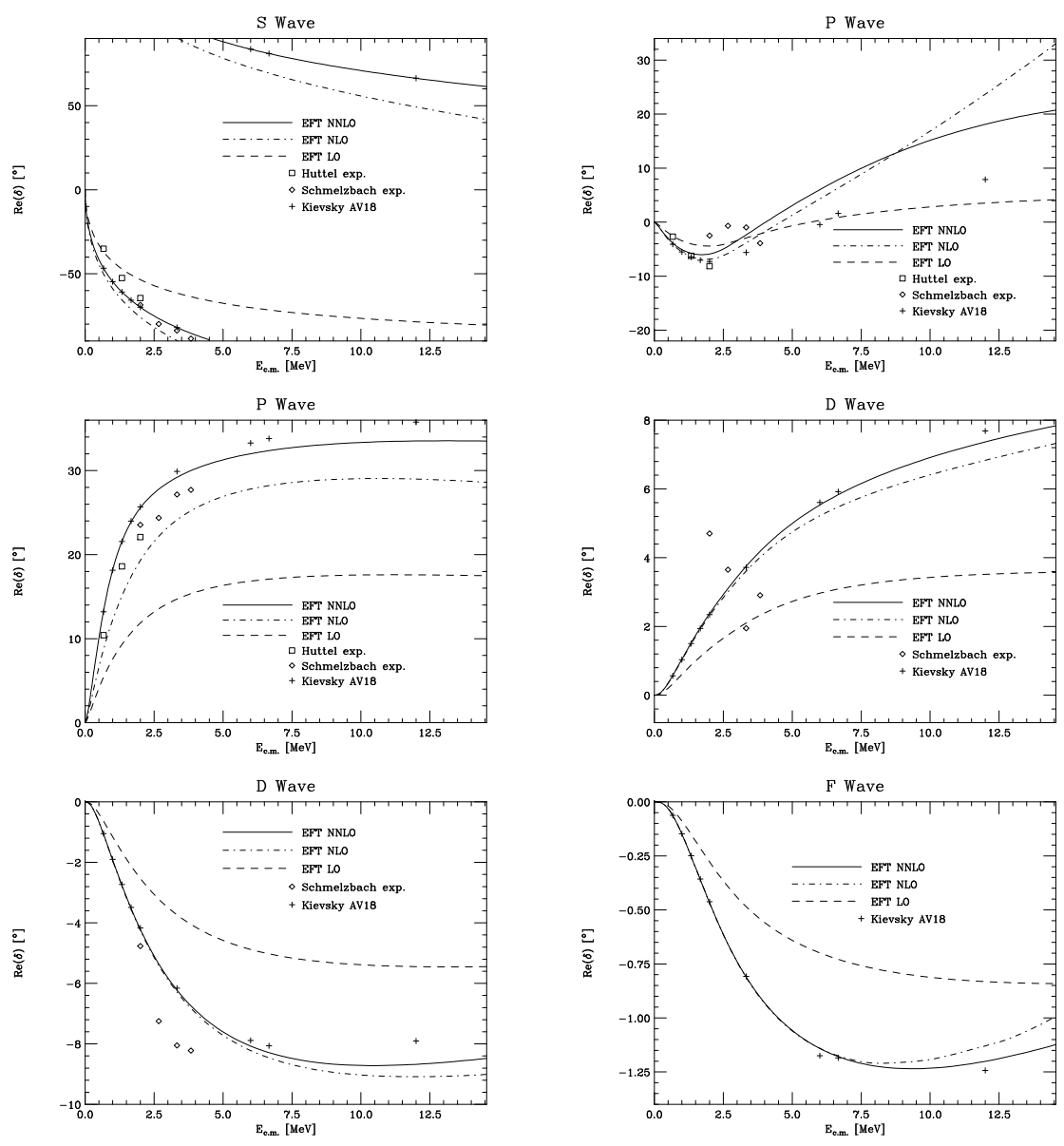

Figure 20: Real part of the first partial waves in the quartet (left) and doublet (right) channels of $n d$ scattering versus the center-of-mass energy. The dashed line is the LO, the dot-dashed the NLO and the solid line the $\mathrm{N}^{2} \mathrm{LO}$ result. 
to the EFT calculation, we expect that any potential model which reproduces these numbers will agree with our results within the estimated error of an $\mathrm{N}^{2} \mathrm{LO}$ calculation, $\sim 4 \% 103$. Higher order calculations in EFT can be easily performed and involve only well known two-body interaction parameters. They too should agree with the latest generation of potential models. Only at the order where three-body forces first contribute will differences between EFT calculations and realistic potential models arise. In some observables, like spin observables in neutron-deuteron scattering, the contribution from three bodyforces appears at lower orders and it is here where differences between EFT and potential models will first become apparent.

\subsection{The Triton Channel}

We know turn to the spin $1 / 2, S$-wave channel. The essential physics point that distinguishes this channel from all the others is that, in the triton- ${ }^{3} \mathrm{He}$ channel, all three nucleons can occupy the same point in space while in all the others the centrifugal barrier and/or Fermi statistics creates an effective repulsion between the three particles at short distances. This suggests that the ultraviolet behavior of this channel should be similar to the case of three spinzero bosons in a $S$-wave that also does not have any short distance repulsion. It turns out that the similarities are more than qualitative and the spin-zero system has all the novel features we want to illustrate. We will discuss first the spin-zero boson case to avoid unessential complications due to the coupled equations in the triton- ${ }^{3} \mathrm{He}$ channel. After the conceptual issues are discussed in the bosonic case we will turn to the triton channel.

The integral equation analogous to Eq. (93) for the three bosons case is

$$
\begin{aligned}
t_{0}(k, p)= & -\frac{y^{2} M}{2 p k} \ln \left(\frac{p^{2}+p k+k^{2}-M E}{p^{2}-p k+k^{2}-M E}\right) \\
& +\frac{2}{\pi} \int_{0}^{\infty} d q q^{2} t_{0}(k, q) \frac{1}{-\gamma+\sqrt{\frac{3 q^{2}}{4}-M E}} K_{0}(q, p),
\end{aligned}
$$

The difference from the quartet case (Eq. (93)) is that the kernel in Eq. (109) has the opposite sign and twice the strength. This is enough to make the Eq. (109) ill-posed and it can be shown that its solution is not unique 906 . Some physical insight into the situation can be obtained by going to coordinate space. For that, let us perform the transformation 104

$$
\chi(r, \rho)=\int_{0}^{\infty} d p t_{0}(k, p) \frac{p \sin (\sqrt{3 / 4} p \rho)}{-\gamma+\sqrt{\frac{3 p^{2}}{4}-M E}} e^{-r \sqrt{\frac{3 p^{2}}{4}-M E}} .
$$


The wave function $\chi(r, \rho)$ satisfies

$$
\left(\frac{\partial^{2}}{\partial r^{2}}+\frac{\partial^{2}}{\partial \rho^{2}}+M E\right) \chi(r, \rho)=0
$$

with the boundary conditions

$$
\chi(r, 0)=0 \quad, \quad \frac{\partial}{\partial r} \chi(0, \rho)-\gamma \chi(0, \rho)+\frac{8}{\sqrt{3}} \frac{1}{\rho} \chi\left(\frac{\sqrt{3}}{2} \rho, \frac{1}{2} \rho\right)=0 .
$$

The last boundary condition is equivalent to Eq. (109). Eqs. (111, 112) could also be obtained by considering the three particle problem in the wave-mechanics formalism and using Jacobi coordinates. The variable $r$ corresponds to the distance between two of the particles and $\rho$ to the distance between the third particle and the center of mass of the other two. Using polar coordinates $R=\sqrt{r^{2}+\rho^{2}}, \tan \alpha=r / \rho$, we can see that this complicated boundary condition is simpler for $R \ll a=1 / \gamma$. In this region Eq. (112) becomes

$$
\frac{\partial}{\partial \alpha} \chi(R, \alpha=0)+\frac{8}{\sqrt{3}} \chi(R, \pi / 3)=0 .
$$

In the region $R \ll a$ we have then a separable problem with the solution

$$
\chi(R, \alpha)=\sum_{i} F_{i}(R) \sin \left(s_{i}(\pi / 2-\alpha)\right),
$$

where the $s_{i}$ are the solutions of

$$
1-\frac{8}{\sqrt{3} s} \frac{\sin \left(s_{i} \pi / 6\right)}{\cos \left(s_{i} \pi / 2\right)}=0 .
$$

Eq. (118) has two imaginary solutions $\pm i s_{0}$, with $s_{0} \simeq 1.006$. The equation for the radial part $F_{0}(R)$ is that of a two dimensional Schroedinger equation with an attractive $1 / R^{2}$ potential

$$
\left(\frac{1}{R} \frac{d}{d R} R \frac{d}{d R}+\frac{s_{0}^{2}}{R^{2}}+M E\right) F_{0}(R)=0 .
$$

As is well known the quantum mechanics problem with an attractive $1 / R^{2}$ potential (or more singular potentials) is not well defined and its hamiltonian is not bounded from below. There are arbitrarily deep bound states so the ground state describes the "fall to the center". Of course this conclusion is not valid as soon as the bound states start probing distances of the order of 
the cutoff. It indicates though that the spectrum will be dependent on short distance physics.

Another way of analyzing this system is to consider the equation describing the asymptotic behavior of the half off-shell amplitude, as we did in the quartet case in Eq. (99). In the case of three bosons we have

$$
p t_{0}(k, p)=\frac{4 \lambda}{\sqrt{3} \pi} \int_{0}^{\infty} d q q t_{0}(k, q) \ln \left(\frac{p^{2}+p q+q^{2}}{p^{2}-p q+q^{2}}\right),
$$

where we introduce a parameter $\lambda$ taking values $\lambda=1$ in the bosonic case and $\lambda=-1 / 2$ in the quartet case. Besides the symmetry under scale transformations $p t_{0}(k, p) \rightarrow \alpha p t_{0}(k, \alpha p)$ Eq. (117) has a symmetry under inversions $p t_{0}(k, p) \rightarrow \frac{\alpha}{p} t_{0}\left(k, \frac{\alpha}{p}\right)$ that will be crucial later. Using the same ansatz $t_{0}(k, p) \sim p^{s-1}$ as before we arrive at an equation determining the asymptotic behavior of $t_{0}(k, p)$

$$
1-\frac{8 \lambda}{\sqrt{3} s} \frac{\sin s \pi / 6}{\cos (s \pi / 2)}=0 .
$$

For $\lambda>\lambda_{c}=\frac{3 \sqrt{3}}{4 \pi} \simeq 0.4135$ Eq. 118) has two imaginary solutions $s= \pm i s_{0}$, with $s_{0} \simeq 1.0062$ in the bosonic case $\lambda=1$. This means that for $p \gg k, \gamma$, $t_{0}(k, p)$ is a linear combination of $p^{i s_{0}-1}$ and $p^{-i s_{0}-1}$ or

$$
t_{0}(k, p)=\frac{A}{p} \cos \left(s_{0} \log (p)+\delta\right) .
$$

The amplitude $A$ is determined by matching the asymptotic solution in Eq. (119) to the full solution in the infrared region. The phase however remains undetermined. This is another way of seeing that there is a one parameter family of solutions to the equation Eq. (109). Of course this undeterminacy is an artifact of extrapolating the low energy effective theory to the ultraviolet region and is eliminated by the introduction of an ultraviolet regulator.

A perturbation theory on the strength of the kernel $(\lambda)$ corresponds to the diagrams on the top line of Fig. 17. The behavior shown in Eq. (119) is missed in all orders of this expansion as can be seen by expanding Eq. (119) in powers of the kernel strength $\lambda$ ( the dependence on $\lambda$ is implicit in $s_{0}$ )

$$
\frac{A}{p} \cos \left(s_{0} \log (p)+\delta\right)=0+0 \lambda+0 \lambda^{2}+\cdots
$$

This explains why a perturbative analysis of the ultraviolet behavior of $t_{0}(k, p)$ misses the oscillatory behavior in Eq. (119). 


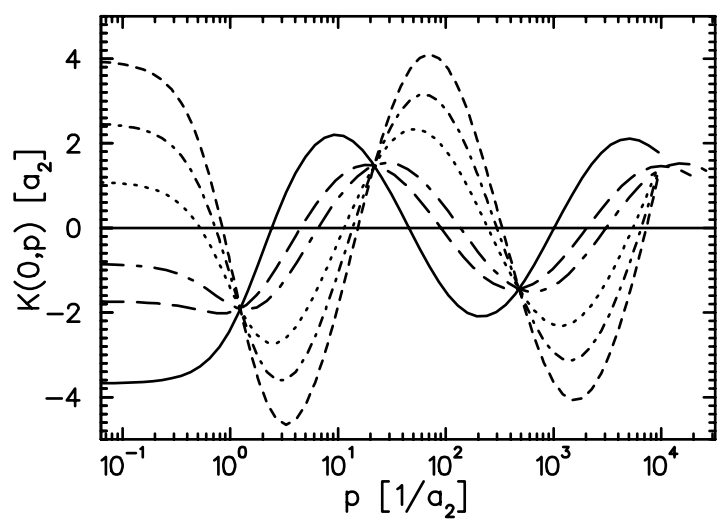

Figure 21: Half off-shell (K-matrix) amplitudes in the bosonic case for different values of the momentum cutoff $\Lambda$ in units of the two-body scattering length $a_{2}$.

In Fig. 21 we can see numerical solutions of Eq. (109) (or more precisely, the K-matrix corresponding to the amplitude $t_{0}(k, p)$ satisfying Eq. (109) with a principal value instead of an $i \epsilon$ prescription) for $k=0$ obtained with different values of the momentum cutoff, $\Lambda$. The presence of $\Lambda$ breaks the inversion symmetry and selects a phase in Eq. (119), and further, different values of $\Lambda$ result in very different values of $t_{0}(k=0, k=0)$. This $\Lambda$ dependence of a physical quantity indicates something is missing in our calculation up to now. As it appears in the three-body amplitudes it should be absorbed by the cutoff dependence of a three-body force. We see now that we were unjustified in dropping the three-body force term from the integral equation in the case of bosons. To reinstate the three-body force shown in Eq. (86) we include the term

$$
\mathcal{L}_{d 3}=-\frac{M H y^{2}}{\Lambda^{2}} d^{\dagger} d N^{\dagger} N
$$

in the Lagrangean and choose $H=D_{0} \frac{\Lambda^{2} \Delta^{2}}{M y^{4}}$. The integration over the field $d$ generates the three-body force in Eq. (86). Graphs containing the three body force are now included as shown in Fig. 22

The new graphs with a three-body interaction change the kernel to

$$
K_{0}(q, p)=\frac{1}{q p}\left[\ln \left(\frac{p^{2}+p q+q^{2}-M E}{p^{2}-p q+q^{2}-M E}\right)+\frac{H}{\Lambda^{2}}\right] .
$$

It is not currently known how to dimensionally regulate the three-body integral equation, and from now on we will use a sharp momentum cutoff instead (some 


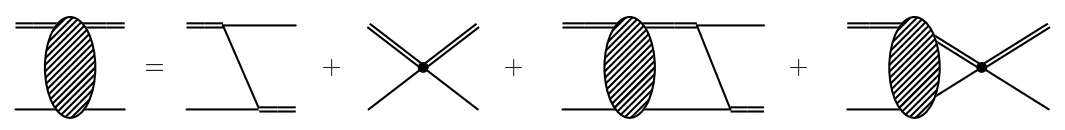

Figure 22: Equation for the three-body amplitude including the three-body force.

progress in this direction was made in the case of a similar equation in 05 ). To be more precise, we will be using two distinct cutoffs: one regulating the twobody loops in the deuteron propagator that is taken to infinity and another regulating the three-body loops. That is why the kernel of the three-body equation contains the renormalized form of the deuteron propagator. This procedure is justified since keeping an unrenormalized form for the deuteron propagator amounts to keeping some sub-leading terms in the kernel that are suppressed by powers of $q / \Lambda$. These terms are relevant only at the cutoff scale $q \sim \Lambda$ and can change only non-universal features, like the value of the bare coupling constants, but not the final physical results.

For $H(\Lambda) \sim 1$ the effect of the three-body force in Eq. (122) is important only for $q \sim \Lambda$ thus our analysis of the asymptotic behavior of the amplitude remains valid in the intermediate region $\gamma, k \ll p \ll \Lambda$. A simple argument now shows that $H(\Lambda)$ can be chosen in such a way as to maintain the lowmomentum part of the amplitude $t_{0}(k, p \ll \Lambda)$ and, in particular, the on-shell point $p=k$, cutoff independent (up to terms of order $k / \Lambda$ ). For that we define a scale $\gamma \ll \mu \ll \Lambda$ and rewrite equation Eq. (109) as

$$
\begin{aligned}
t_{0}^{\Lambda}(k, p) & =K(k, p)+\frac{2}{\pi} \int_{0}^{\mu} d q q^{2} t_{0}^{\Lambda}(k, q) K_{0}(q, p) \\
& +\frac{2}{\pi} \int_{\mu}^{\Lambda} d q q^{2} t_{0}^{\Lambda}(k, q)\left(K_{0}(q, p)+\frac{H(\Lambda)}{\Lambda^{2}}\right)+\mathcal{O}(p / \Lambda, \gamma / \Lambda)
\end{aligned}
$$

The superscript $\Lambda$ reminds us that $t_{0}^{\Lambda}(k, p)$ is the solution of the integral equation with a particular cutoff $\Lambda$. Taking another cutoff $\Lambda^{\prime}$ we can write the equation for $t_{0}^{\Lambda^{\prime}}(k, p)$ as

$$
\begin{aligned}
t_{0}^{\Lambda^{\prime}}(k, p) & =K(k, p)+\frac{2}{\pi} \int_{0}^{\mu} d q q^{2} t_{0}^{\Lambda^{\prime}}(k, q) K_{0}(q, p) \\
& +\frac{2}{\pi} \int_{\mu}^{\Lambda} d q q^{2} t_{0}^{\Lambda^{\prime}}(k, q)\left(K_{0}(q, p)+\frac{H(\Lambda)}{\Lambda^{2}}\right)
\end{aligned}
$$




$$
\begin{aligned}
& +\frac{2}{\pi} \int_{\Lambda}^{\Lambda^{\prime}} d q q^{2} t_{0}^{\Lambda^{\prime}}(k, q)\left(\frac{H\left(\Lambda^{\prime}\right)}{\Lambda^{\prime 2}}+\frac{1}{q^{2}}\right) \\
& +\frac{2}{\pi} \int_{\mu}^{\Lambda} d q q^{2} t_{0}^{\Lambda^{\prime}}(k, q)\left(\frac{H\left(\Lambda^{\prime}\right)}{\Lambda^{\prime 2}}-\frac{H(\Lambda)}{\Lambda^{2}}\right)+\mathcal{O}\left(\frac{p}{\Lambda}, \frac{\gamma}{\Lambda}\right) .
\end{aligned}
$$

If $H(\Lambda)$ can be chosen in such a way as to make the terms in the last two lines of Eq. (124) vanish, the equations satisfied by $t_{0}^{\Lambda}(k, q)$ and $t_{0}^{\Lambda^{\prime}}(k, q)$ are the same and, since the equation with a finite cutoff has an unique solution, the amplitudes are the same. Using the asymptotic form of the amplitudes

$$
t_{0}^{\Lambda}(k, \gamma \ll p \ll \Lambda)=t_{0}^{\Lambda^{\prime}}\left(k, \gamma \ll p \ll \Lambda^{\prime}\right)=\frac{A}{p} \cos \left(s_{0} \log (p) / \bar{\Lambda}\right),
$$

where $\bar{\Lambda}$ is an arbitrary scale fixing the asymptotic phase we find an approximate form for $H(\Lambda)$

$$
H(\Lambda)=-\frac{\sin \left(s_{0} \log \left[\Lambda / \bar{\Lambda}-\arctan \left[1 / s_{0}\right]\right)\right.}{\sin \left(s_{0} \log [\Lambda / \bar{\Lambda}]+\arctan \left[1 / s_{0}\right]\right)}
$$

which assures $\Lambda$ independence. The precise form of $H(\Lambda)$ in Eq. (126) might dependent on the particular shape of the cutoff, but its qualitative behavior, in particular the existence of a limit cycle in RG flow, are general.

We can check numerically the argument leading to Eq. (126). For this we can fix (arbitrarily) the value of the three-body scattering length and determine numerically the value of $H(\Lambda)$ needed to reproduce this same scattering length for any given value of $\Lambda$. This can always be done since we have one free parameter $(H(\Lambda))$ to fit another (the three-body scattering length). Using these determined values of $H(\Lambda)$ we can check that the amplitudes at finite $k$ are indeed cutoff independent (up to terms of order $k / \Lambda, \Lambda a$ ). The result of this procedure is shown as the dots in Fig. 23. The agreement with Eq. (126) lends support to the arguments leading to it.

The appearance of a new parameter (that can be taken to be the value $H(\Lambda)$ at some particular value of $\Lambda$ or the scale $\bar{\Lambda}$ ) that is not determined by two-body physics is the main result of this analysis. It implies that different models having the same two-body scattering length can have quite different three-body physics. This is shown in a striking manner by plotting the prediction of a number of different models with very similar low energy $\left(p<m_{\pi}\right)$ two-body physics for two three-body observables like, for instance, the threebody bound state energy $\left(B_{3}\right)$ and the scattering length of a particle colliding with the bound state of two others $\left(a_{3}\right)$ 106. It is known from the numerical 


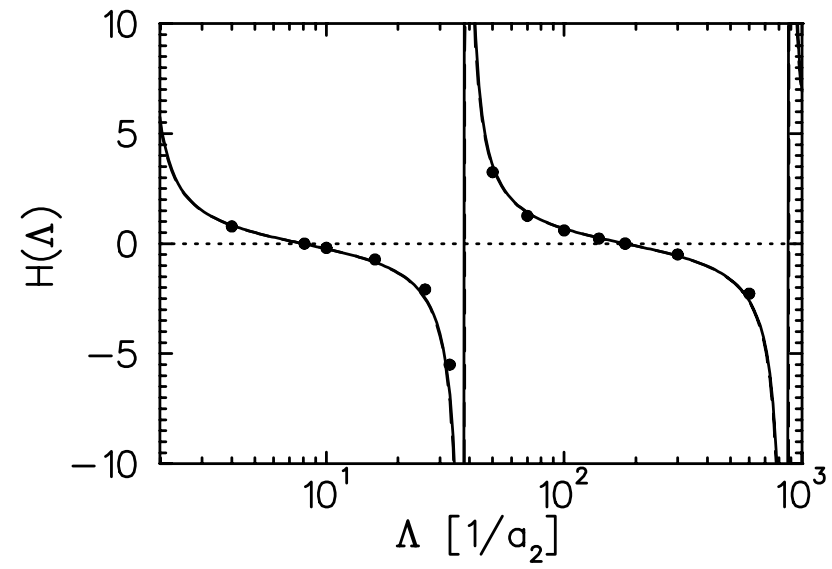

Figure 23: Running of the three-body force around a limit cycle. The solid line shows Eq. 126) and the dots the numerical procedure described in the text.

studies of potential models that those predictions can differ wildly among different models, but not to scatter all over the $a_{3} \times B_{3}$ plane either. They tend to lie along a reasonably well defined line (Phillips line, see Fig. 24). For the point of view discussed here each point on the Phillips line correspond to a different value of the three-body parameter $\bar{\Lambda}$.

The existence of the Phillips line is usually understood as the result of the dependence of the three-body observables on the particylar off-shell extension of the two-body data described by the different potentials 107 . This is equivalent to the explanation discussed above since field redefinitions change both offshell behavior of amplitudes and strengths of three-body forces. Looking at those "off-shell ambiguities" as values of short distance counterterms has the advantage of being a systematic way of mapping them out and estimating their sizes. In particular it becomes clear that the "off-shell ambiguity" related to the three-body bound state cannot be fixed by two-body processes involving external currents since those are related to different counterterms.

We now have the understanding necessary to tackle the triton- ${ }^{3} \mathrm{He}$ channel, described by Eq. (108) 108. There is an interesting limit where Eqs. (108) decouple. The two spin and isospin states of the nucleons can be put in a $\mathbf{4}$ representation of spin-flavor $S U(4)$. This symmetry is broken in leading order by the difference between the scattering lengths in the ${ }^{3} S_{1}(\gamma)$ and ${ }^{1} S_{0}$ (a) channels but is an approximate symmetry of the system when the typical momenta is $Q \gg \gamma, 1 / a$ since in this case we can take $\gamma \simeq 1 / a \simeq 0$. This approximate symmetry is observed in light nuclei and has interesting conse- 


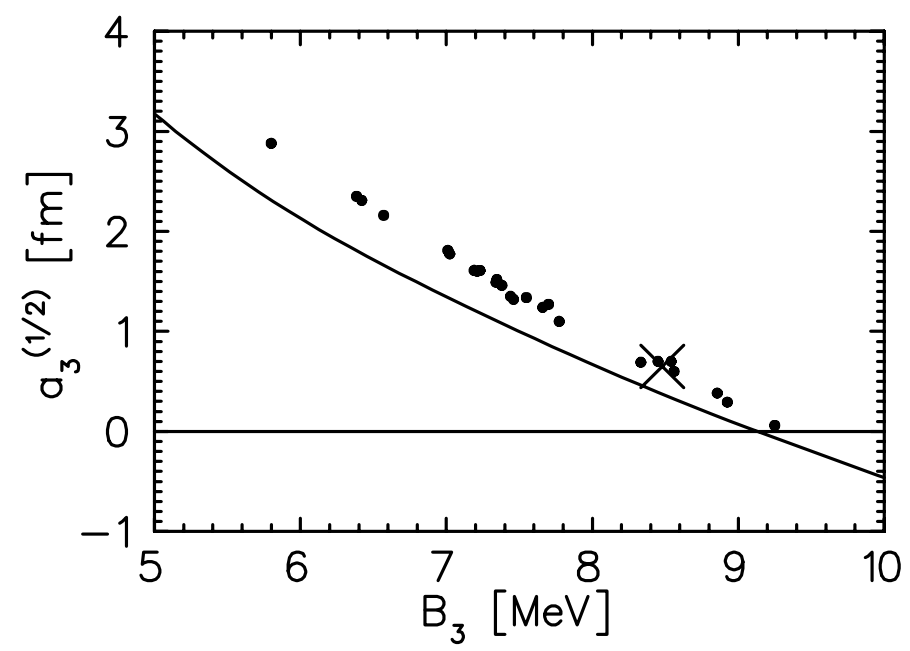

Figure 24: Results for the doublet $S$-wave neutron-deuteron scattering length for different models having very similar two-body scattering lengths and effective ranges, denoted by the dots. The solid line is the EFT prediction over a range of values for $\bar{\Lambda}$. The cross indicates the experimental value.

quences 109.110. In the $S U(4)$ limit the system of equations Eq. (108) break up into two equations: one for the sum $t_{+}(k, p)=t_{d}(k, p)+t_{t}(k, p)$ and the other for the difference $t_{-}(k, p)=t_{d}(k, p)-t_{t}(k, p)$. The three-body force in Eq. 866) contributes only to the bosonic-like equation for $t_{-}(k, p)$. It is actually the only three-body force with no derivatives one can write down, as a simple argument shows. Fermi statistics mandates that the three nucleons should be in a completely antisymmetric representation of $S U(4)$. Consequently, the three-body force transforms as $\mathbf{4} \otimes \overline{\mathbf{4}}=\mathbf{1} \oplus \mathbf{1 5}$, but only the singlet is separately invariant under the spin and isospin subgroups. The three-body force in Eq. (86) is generated by the inclusion of the term

$$
\begin{aligned}
\mathcal{L}_{d, 3} & =\frac{2 M H(\Lambda)}{\Lambda^{2}}\left[y_{d}^{2} N^{\dagger}\left(d^{i} \sigma^{i}\right)^{\dagger}\left(d^{j} \sigma^{j}\right) N+y_{t}^{2} N^{\dagger}\left(t^{a} \tau^{a}\right)^{\dagger}\left(t^{b} \tau^{b}\right) N\right. \\
& \left.+\frac{1}{3} y_{t} y_{s}\left[N^{\dagger}\left(d^{i} \sigma^{i}\right)^{\dagger}\left(t^{a} \tau^{a}\right) N+h . c .\right]\right]
\end{aligned}
$$

Upon integrating the field $d^{i}$ and $t^{a}$ out we recover Eq. (86). Fitting $\bar{\Lambda}$ to the value of the experimental doublet scattering length $a_{1 / 2}^{\text {exp }}$ we arrive at the solif curve in Fig. 25. Also shown in Fig. 25 are the phase shift analysis of 98.99 and the experimental value of the doublet scattering length. Using those 


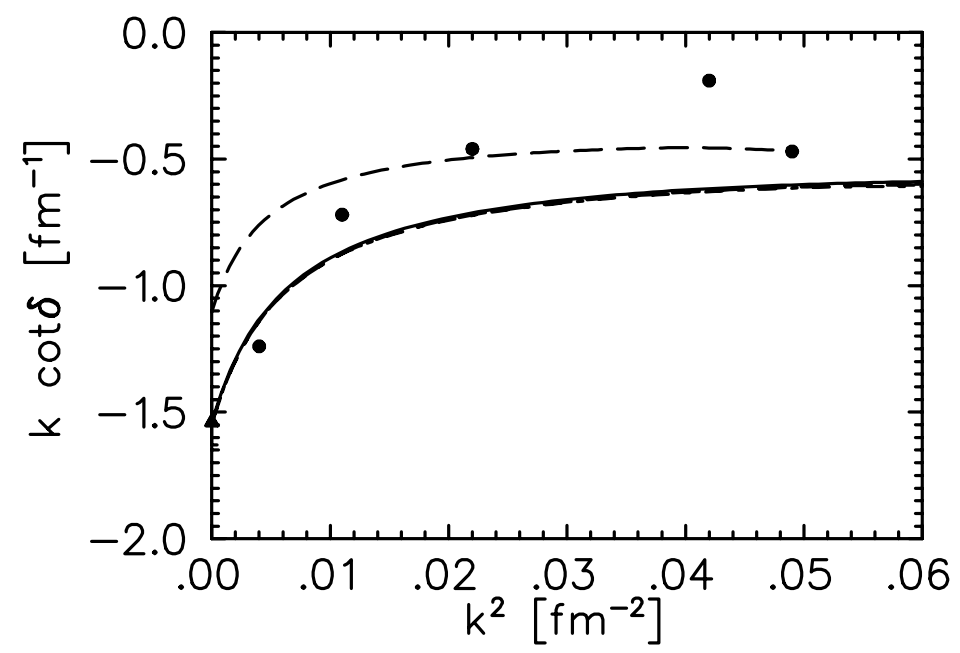

Figure 25: Doublet $S$-wave neutron-deuteron phase shifts.

parameters the prediction for the triton binding energy is of $B_{3}=8.03 \mathrm{MeV}$, to be compared with the experimental value $B_{3}^{\text {exp }}=8.48 \mathrm{MeV}$. The dashed line was obtained by using the experimental value of the two-body scattering length, instead of the deuteron binding energy, to fix ${ }^{\star} C_{0}^{\left({ }^{3} S_{1}\right)}$, while keeping the three-body force constant. Both procedures differ only by higher orders terms so their difference provides an estimate of the higher order corrections. Comparison with experiment suggests that the low energy expansion in the triton is under control since the errors are within the expected value for a LO calculation. A more solid statement can be made only when higher order calculations become available. A natural question to ask then is whether the three-body force needed to give the triton its experimental binding energy is "natural". For cutoffs of the order $\Lambda \simeq m_{\pi}, H\left(\simeq m_{\pi}\right) \simeq 1$ suggesting that the relative shallowness of the triton does not require any further fine tuning besides that in the two-body sector.

Compared to the two-body sector the study of the three-nucleon problem with EFT methods is still in its infancy. A number of conceptual and technical problems have to be understood before high order calculations can be performed. Among them are the complete power counting to all orders and the scaling of the sub-leading three-body forces (some recent progress was report in Ref.111). 


\section{$5 \quad N N$ Interactions at Higher Energies}

In order to make progress toward describing multi-nucleon systems, such as nuclei, an EFT must be constructed that will describe the $N N$ system at momenta $|\mathbf{p}| \gtrsim m_{\pi}$. It is obvious that one must include the $\pi$-field explicitly in this kinematic region. However, a power-counting must be developed that is both systematic and convergent. In what follows we will describe the two different power-counting schemes that currently exist in this kinematic regime. Weinberg's power-counting 8 is based on a momentum and chiral expansion of the $N N$ potential, in which the momentum independent, local four-nucleon interaction and one-pion-exchange (OPE) both enter at leading order. Higher derivative operators and the light-quark mass matrix occur at higher orders and are inserted in perturbation theory. Calculations that have been performed to date, indicate that the perturbative expansion appears to be converging in the two-nucleon sector, as we shall discuss subsequently, however, there are formal issues that remain to be resolved. The jury is still out in the three-nucleon sector, but encouraging results are being obtained. In contrast, KSW power-counting provides an explicit framework in which to systematically expand S-matrix elements as opposed to an expansion of the (unobservable) potential. Calculations are renormalization scale independent order-by-order in the expansion parameter. However, acalculation of S-wave $N N$ scattering at $\mathrm{N}^{2} \mathrm{LO}$ by Fleming, Mehen and Stewar 112 indicates that, in fact, treating the pions perturbatively is not converging for momenta $|\mathbf{p}| \gtrsim m_{\pi} / 2$. They were able to identify the one large contribution that occurs at $\mathrm{N}^{2} \mathrm{LO}$, and found that it persists in the $m_{q} \rightarrow 0$ chiral limit. Hopefully this problem can be understood and solved in the near future.

This is essentially the current status of the field. In what follows both power-countings will be discussed, both their strengths and currently understood weaknesses. Emphasis will be placed on the Weinberg counting due to its present numerical successes and lack of numerical failures, despite some unresolved formal issues.

\section{Perturbative $\pi$-Exchange and its Problems}

Let us recall how pions couple to nucleons. The Lagrange density describing the interactions between pions and nucleons is

$$
\mathcal{L}=\frac{f_{\pi}^{2}}{4} \operatorname{Tr} D_{\mu} \Sigma D^{\mu} \Sigma^{\dagger}+\frac{i g_{A}}{2} N^{\dagger} \sigma \cdot\left(\xi \mathbf{D} \xi^{\dagger}-\xi^{\dagger} \mathbf{D} \xi\right) N+\cdots
$$


where the pion fields are contained in a special unitary matrix,

$$
\Sigma=\xi^{2}=\exp \frac{\sqrt{2} i \Pi}{f}, \quad \Pi=\left(\begin{array}{cc}
\pi^{0} / \sqrt{2} & \pi^{+} \\
\pi^{-} & -\pi^{0} / \sqrt{2}
\end{array}\right),
$$

with $f=93 \mathrm{MeV}$ and $g_{A}=1.26$ is the axial coupling constant. The ellipses represent operators involving more insertions of the light quark mass matrix, meson fields, and spatial derivatives. The leading-order contribution to the $N N$ potential due to the exchange of a single "potential pion" $\|$ is:

$$
V_{\pi}(\mathbf{q})=-\frac{g_{A}^{2}}{4 f_{\pi}^{2}} \frac{\left(\mathbf{q} \cdot \sigma^{1}\right)\left(\mathbf{q} \cdot \sigma^{2}\right)}{|\mathbf{q}|^{2}+m_{\pi}^{2}} \vec{\tau}_{1} \cdot \vec{\tau}_{2}
$$

where $\sigma^{k}$ denotes the spin operator acting on the $k$ th nucleon. It is clear that this interaction does not have any dependence upon the renormalization scale $\mu$. Since the $C_{0}$ operator scales as $1 /(M \mu)$ it would appear that as the system is evolved into the infrared, the short-range contact interaction will dominate over one-pion exchange. From the standpoint of conventional nuclear physics this is a shocking claim. The tensor force in the ${ }^{3} S_{1}-{ }^{3} D_{1}$ channel is known to be strong, and this casts doubt on the applicability of a perturbative treatment of the pion exchanges. The question that must be addressed is what is the kinematic regime where this hierarchy is actually present. The observation that there is a kinematic regime where pions can betreated in perturbation theory was first made by Kaplan, Savage and Wise 31,30 (KSW), and the associated power-counting worked out in these papers is commonly known as KSW-counting. KSW-counting has the very attractive feature that analytic expressions for any scattering amplitude can be found.

\subsection{NN Scattering with Perturbative Pions}

The $N N$ scattering amplitude has now been computed up to $\mathrm{N}^{2} \mathrm{Ld} 12$ in KSW counting. The LO contribution to the amplitude is exactly the same as in the pionless theory, since, as mentioned above, the pion scales as $Q^{0}$, while $C_{0}$, and the whole bubble chain contributing to $\mathcal{A}^{(-1)}$ scale as $Q^{-1}$. Thus, once more:

$$
\mathcal{A}_{-1}=-\frac{C_{0}^{\left({ }^{1} S_{0}\right)}}{1+C_{0}^{\left({ }^{1} S_{0}\right)} \frac{M}{4 \pi}(\mu+i p)} \text {. }
$$

${ }^{i}$ The variation of the pion propagator due to the energy transfer between the two nucleons is negligible compared to the variation due to the three-momentum transfer. 


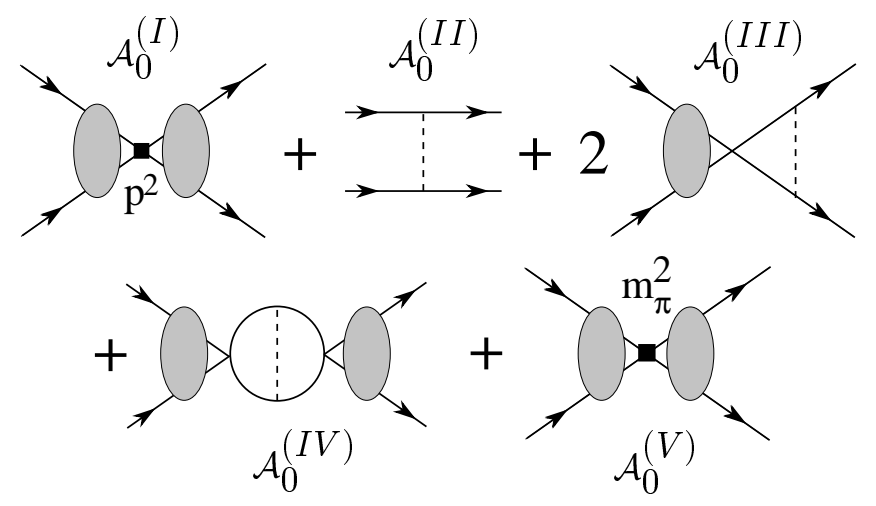

Figure 26: Feynman diagrams contributing to $N N$ scattering in the ${ }^{1} S_{0}$ channel.

Meanwhile, the Feynman diagrams contributing to the NLO amplitude are shown in Fig. 26 and their contribution is the sum $\mathcal{A}_{0}=\mathcal{A}_{0}^{(I)}+\mathcal{A}_{0}^{(I I)}+$ $\mathcal{A}_{0}^{(I I)}+\mathcal{A}_{0}^{(I V)}+\mathcal{A}_{0}^{(V)}$. The local operators at this order involve either two spatial derivatives, with coefficient $C_{2}^{\left({ }^{1} S_{0}\right)}$, or one insertion of the light quark mass matrix, with coefficient $D_{2}^{\left({ }^{1} S_{0}\right)}$. Thus, the amplitude is

$$
\begin{aligned}
\mathcal{A}_{0}^{(I)} & =-C_{2}^{\left({ }^{1} S_{0}\right)} p^{2}\left[\frac{\mathcal{A}_{-1}}{C_{0}^{\left({ }^{1} S_{0}\right)}}\right]^{2}, \mathcal{A}_{0}^{(I I)}=-\frac{g_{A}^{2}}{4 f^{2}}\left(1-\frac{m_{\pi}^{2}}{4 p^{2}} \ln \left(1+\frac{4 p^{2}}{m_{\pi}^{2}}\right)\right), \\
\mathcal{A}_{0}^{(I I I)} & =-\frac{g_{A}^{2}}{2 f^{2}}\left(\frac{m_{\pi} M \mathcal{A}_{-1}}{4 \pi}\right)\left(\frac{\mu+i p}{m_{\pi}}-i \frac{m_{\pi}}{2 p} X_{\pi}\right), \\
\mathcal{A}_{0}^{(I V)} & =-\frac{g_{A}^{2}}{4 f^{2}}\left(\frac{m_{\pi} M \mathcal{A}_{-1}}{4 \pi}\right)^{2}\left(\left(\frac{\mu+i p}{m_{\pi}}\right)^{2}-X_{\pi}-\log \left(\frac{m_{\pi}}{\mu}\right)+1\right) \\
\mathcal{A}_{0}^{(V)} & =-D_{2}^{\left({ }^{1} S_{0}\right)} m_{\pi}^{2}\left[\frac{\mathcal{A}_{-1}}{C_{0}^{\left({ }^{1} S_{0}\right)}}\right]^{2}, \\
X_{\pi} & =\frac{1}{2} \ln \left(1+\frac{4 p^{2}}{m_{\pi}^{2}}\right)-i \tan ^{-1}\left(\frac{2 p}{m_{\pi}}\right) .
\end{aligned}
$$

The resulting ${ }^{1} S_{0}$ phase shifts at LO, NLO 30 and $\mathrm{N}^{2} \mathrm{LO} 112$ are shown in Fig. 27. It should be noted that these results are for a particular fitting procedure and appear to be converging very well. Detailed discussions of various fitting procedures, and whether the pion can truly be treated in perturbation theory 
in the ${ }^{1} S_{0}$ channel, can be found in Refs. $113,114,115,110,11,118,119$.

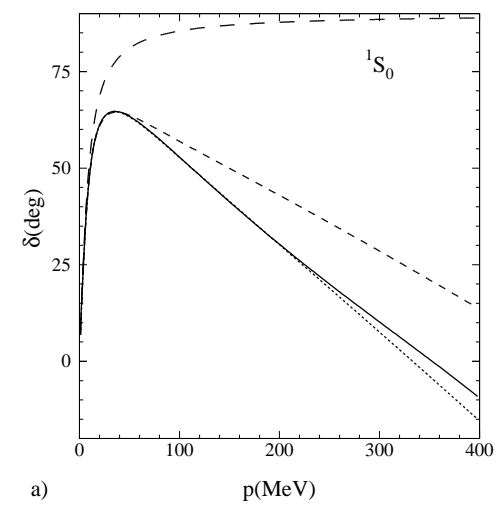

Figure 27: Fit to the ${ }^{1} S_{0}$ phase shift $\delta$. The solid line is the Nijmegen fit 1 to the data. The long dashed, short dashed, and dotted lines are the LO, NLO, and $\mathrm{N}^{2} \mathrm{LO}$ results respectively.

A similar calculation has been performed in the ${ }^{3} S_{1}-{ }^{3} D_{1}$ coupled channels but we will not present the expressions for the amplitudes here 30.12. The resultant function yields the ${ }^{3} S_{1}$ phase shifts shown in Fig. 28. From Fig. 28
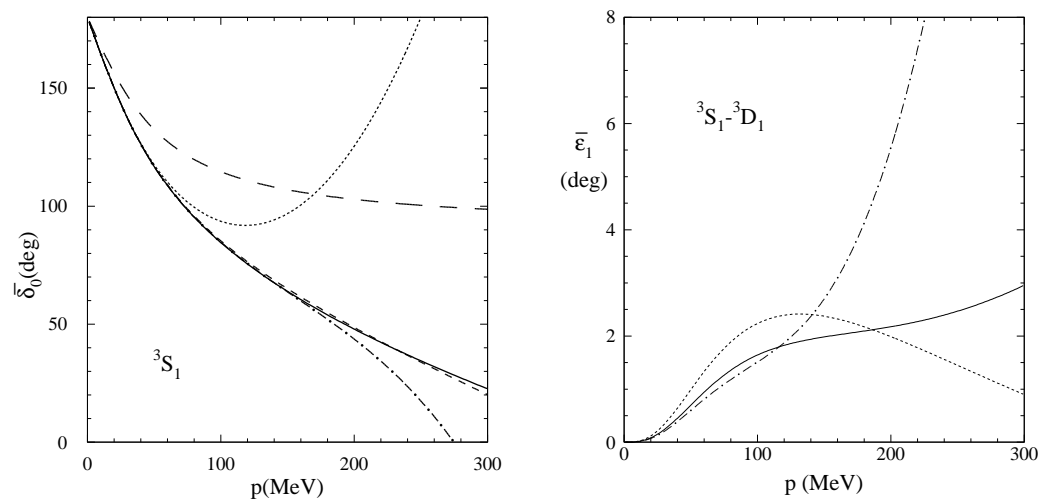

Figure 28: The ${ }^{3} S_{1}-{ }^{3} S_{1}$ phase shift, $\bar{\delta}_{0}$ and ${ }^{3} S_{1}-{ }^{3} D$ mixing parameter, $\bar{\varepsilon}_{1}$ for $N N$ scattering. The solid line is the Nijmegen multi-energy fit 4 , the long dashed line is the LO effective field theory result, the short dashed line is the NLO result, and the dotted line is the $\mathrm{N}^{2} \mathrm{LO}$ result. The dash- line shows the result of including the parameter $\zeta_{5}$ (defined in Ref.112) which is higher order in the power counting

it is clear that the $\mathrm{N}^{2} \mathrm{LO}$ phase shift is deviating significantly from the data 
at momenta $|\mathbf{p}| \gtrsim m_{\pi} / 2$. Given that one expects the perturbative series to converge to the data at successively higher orders, it would appear that the KSW-expansion is in trouble.

This conclusion is reinforced by looking at the ${ }^{3} S_{1}-{ }^{3} D_{1}$ mixing parameter, $\bar{\varepsilon}_{1}$, defined above. This observable has also been computed up to $\mathrm{N}^{2} \mathrm{~L} 44$. The results at $\mathrm{LO}, \mathrm{NLO}$ and $\mathrm{N}^{2} \mathrm{LO}$ are shown in Fig. 28 .

The curves in Fig. 28 suggest that KSW power-counting is failing in the ${ }^{3} S_{1}-{ }^{3} D_{1}$ coupled channels. The origin of this failure in the ${ }^{3} S_{1}$-channel at $\mathrm{N}^{2} \mathrm{LO}$ has been tracked down to a term that survives in the chiral limit

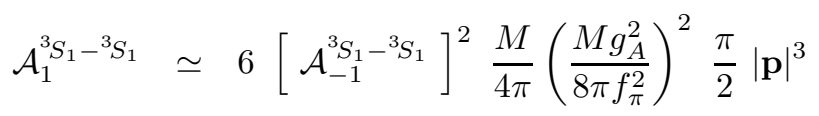

where $\mathcal{A}_{-1}^{3} S_{1}-{ }^{3} S_{1}$ is the LO ${ }^{3} S_{1}-{ }^{3} S_{1}$ amplitude. The $\mathrm{N}^{2} \mathrm{LO}$ amplitude in Eq. (133) is large because of the coefficient of 6 which is to be compared with the expansion parameter of $\sim 1 / 3$. The fact that this correction survives in the chiral limit indicates that it comes from the short distance part of potential pion exchange. The problem one encounters in this coupled channel is really due to the large strength of the tensor force. Of course, in the ${ }^{3} S_{1}-{ }^{3} S_{1}$ amplitude this problem only arises at $\mathrm{N}^{2} \mathrm{LO}$, since that is where two-pion-exchange diagrams first appear. A method to deal with the large non-analytic terms of Eq. (133) has yet to be uncovered, although there are hopes that the solution will come from a better understanding of the renormalization of singular potentials.

The phenomenology of KSW power-counting in the two-nucleon sector at relatively low orders has been extensively explored in a number of processes which are of current experimental interest. However, none of the calculations of these processes were carried out to an order sufficiently high that the strong $\mathrm{N}^{2} \mathrm{LO}$ correction to ${ }^{3} S_{1}$ scattering played a role. Therefore, it may be that these results represent only low-order terms in a series which ultimately does not converge. None of the results obtained using KSW power-counting can be taken too seriously until the convergence issue is resolved. The fact that the KSW power-counting allows for all calculations to be performed analytically with dimensional regularization means that it is very easy to perform gauge invariant calculations for processes involving photons and $\mathrm{ther}$ gauge fields. In particular, $e d$ scattering has been calculated up to NLOE2, $\gamma d \rightarrow \gamma d$ Compton scattering has been computed (both for unpolarized and tensor-polarized targets) up to NLO 120121 , the polarizabilities of the deuteron have been computed to high-orders 122, parity violation in $n p \rightarrow d \gamma$ radiative capture has been explored 123 , and also parity violation in ed scattering due to the deuteron anapole moment has been investigated 124.125. 


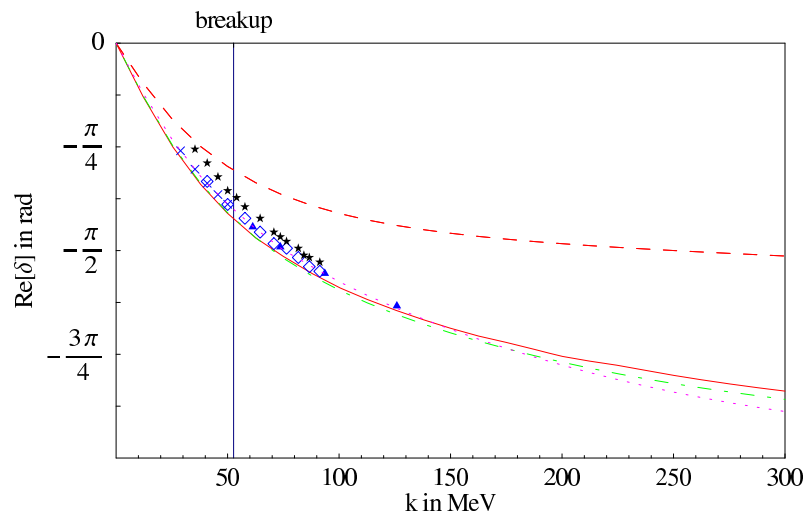

Figure 29: Neutron-deuteron phase shifts in the S-wave quartet in LO (dashed), NLO (full), NLO in the pionless theory (dot-dashed) and $\mathrm{N}^{2} \mathrm{LO}$ in the pionless theory (dotted). Also shown are the results of PSA of neutron-deuteron (squares) and proton-deuteron scattering (stars).

Calculations with perturbative pions have also been performed in the three-body sector 126 . In the $J=3 / 2$, S-wave channel there are no unknown coefficients appearing at NLO, and thus there is no dependence of the result on any fitting procedure. The LO calculation involves only two-body contact interactions. The NLO corrections come from one potential pion exchange, taken in perturbation theory. They are of two kinds: corrections to the deuteron propagator involving only two nucleons and true three-body diagrams, where the three-nucleons participate. The diagrams have to be computed numerically since the half off-shell amplitude $t_{0}(k, p)$ (solution of Eq. (93)) is known only numerically. As discussed before, the contribution from the tensor force vanishes in linear order in the $\mathrm{S}$-wave so there is no contribution from the tensor part of the one-pion exchange. The results for the phase shift are shown in Fig. 29, together with the extrapolation from the result obtained with the theory without pions and phase shift analysis, where it is available. The results indicate convergence up to very high energies but are barely distinguishable from the pionless theory. This is probably a consequence of the high anomalous dimension of the amplitude $t_{0}(k, p)$, that decays to zero very rapidly at large $p$.

\section{Don't Mess with Texas: the Weinberg Program for Physics of the Two-Nucleon System}




\subsection{The Proposal}

One way of dealing with the non-perturbative nature of the pion exchanges uncovered by higher-order calculations in KSW counting is to follow Weinberg's original proposal for the $N N$ system 1 . In this proposal one uses baryon $\chi P T$ to compute the $N N$ potential $V_{N N}$ perturbatively. The $\mathrm{S}$-matrix is then generated by the Schrödinger equation. In this approach, power-counting is not manifest in the amplitudes for individual processes, but there is a naive $a$ priori estimate of the error in a particular calculation, which must be checked a posteriori.

Weinberg's separation of all graphs contributing to some process in the $N N$ system into graphs which are "two-particle reducible" and those which are not makes power-counting much simpler. Since, by definition, the "two-particle irreducible" (2PI) graphs do not contain the low-energy scales $B$ (deuteron binding energy) and $p^{2} / M$. The 2PI graphs are precisely those where the energy of all internal legs is of order $m_{\pi}$. It follows immediately that the $2 \mathrm{PI}$ graphs have the power-counting of ordinary $\chi \mathrm{PT}$.

The "Weinberg program" for calculating properties of the $N N$ system in $\chi \mathrm{PT}$ then centers around the construction of these 2PI graphs. Its implementation has three stages. Firstly, we must consider the case where the $N N$ system is not subject to any external probes, in which case the $N N$ irreducible diagrams just yield the potential $V$ (e.g. the left-hand graph of Fig. 30.) Constructing an $N N$ potential using the rules of $\chi \mathrm{PT}$ will then yield the $N N$ interaction, $V^{(n)}$, up to a given order $n$ in the chiral power counting.

The second step is to obtain the full $N N$ t-matrix by iterating these irreducible graphs together with the free two-nucleon Green's function, $G_{0}$ :

$$
G_{0}(E ; \mathbf{p})=\frac{1}{E-\frac{\mathbf{p}^{2}}{M}+i \eta} .
$$

( $\eta$ is a positive infinitesimal.) This free Green's function can, of course, have a small energy denominator that does not obey the usual $\chi \mathrm{PT}$ power-counting. Iteration of $V$ with $G_{0}$ gives contributions to the t-matrix such as the graph shown on the right-hand side of Fig. 30. This simply means that we follow the usual practice in the $N N$ system, and construct the full $N N$ t-matrix, $T$, from the chiral $N N$ interaction $V^{(n)}$, and the free two-nucleon Green's function $G_{0}$, via the Lippmann-Schwinger (or, equivalently, the Schrödinger) equation

$$
T=V^{(n)}+V^{(n)} G_{0} T .
$$

This point of view is similar to that seen when low-energy effective actions are derived using Wilsonian RG arguments (see Refs. 127,28 for more 

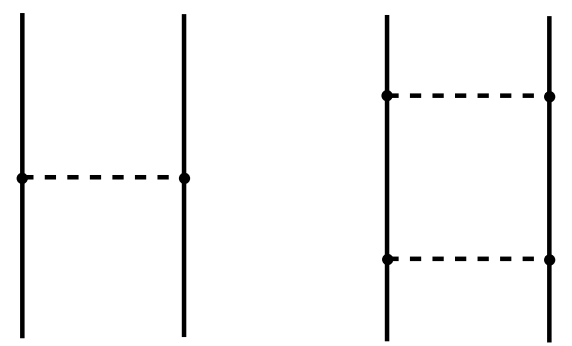

Figure 30: One- and two-pion exchange graphs. One-pion exchange is in the potential and (uncorrelated) two-pion exchange is generated by solving the Schrödinger equation.

explicit investigations of this connection). These techniques are widely applied, and have, for instance, been used to obtain improved actions for lattice gauge theory. In all cases the effective action is obtained using a perturbation theory in the ratio of low-energy scales to the Wilsonian RG cutoff scale. That Lagrangian is then solved to all orders. The error of such a calculation is expected to be dominated by the lowest-dimensional operator omitted from the calculation. This allows one to make an a priori estimate of the error, which can then be verified a posteriori 10 . In the same way the Weinberg program employs $\chi P T$ to generate a nonrelativistic, particle-number-conserving Hamiltonian for the nuclear system. This Hamiltonian is valid up to a given order in the chiral expansion and can be employed in any many-nucleon system at low energy. Once written down it should be solved to all orders.

With the $N N$ t-matrix in hand implementation of the third step of the Weinberg program can proceed. In this stage we consider the interaction of some external probe: a pion, a photon, or some weakly-interacting particle, with the $N N$ system. Provided this probe carries momentum of order $m_{\pi}$ it can have its interaction with the $N N$ system represented as a sum of irreducible diagrams which forms a kernel $K_{\text {probe }}$ for the process of interest. Again, the power-counting of $\chi \mathrm{PT}$ applies to this kernel, since all nucleon energies appearing in it are, by definition, of order $m_{\pi}$. Naturally, this is only true if the external probe energy and momentum are both of $O\left(m_{\pi}\right)$, but, subject to that limitation, $\chi \mathrm{PT}$ allows us to calculate the kernel $K_{\text {probe }}$ up to a given order in the chiral expansion. The full amplitude for this reaction is then found by multiplying this kernel $K_{\text {probe }}$ by factors which take account of the initial and final-state interactions of the $N N$ pair. In other words the amplitude is

$$
\mathcal{A}=\left(1+T G_{0}\right) K_{\text {probe }}\left(1+G_{0} T\right)
$$


Here $T$ is calculated from Eq. (135), using a $V^{(n)}$ which is also computed in $\chi \mathrm{PT}$. For consistency the expansion for the interaction with the external probe should be taken to the same chiral order as the order $n$ used in the chiral expansion of the $N N$ potential $V^{(n)}$. Once this is done the result for the amplitude $\mathcal{A}$ should be the unambiguous prediction of $\chi \mathrm{PT}$ for the process of interest up to that order in the chiral expansion.

Up until now our discussion has been completely general, and could apply to the scattering of particles from the $N N$ bound state, the deuteron, the break-up of the deuteron by a probe (e.g. $\gamma d \rightarrow n p$ ) or to the emission of particles from the $N N$ state (e.g. proton-proton bremsstrahlung). However, if we are especially interested in probes of the deuteron we may take the residue of Eq. (136) at the deuteron pole in both the initial and final-state. We then see that the amplitude for the interaction of the probe with the deuteron is

$$
\mathcal{A}=\left\langle\psi\left|K_{\text {probe }}\right| \psi\right\rangle
$$

where the wave function $|\psi\rangle$ is to be calculated by solving the Schrödinger equation for the potential $V^{(n)}$ :

$$
\left(E-\frac{p^{2}}{M}\right)|\psi\rangle=V^{(n)}|\psi\rangle .
$$

This program is a close relative of that pursued by nuclear physicists in the $N N$ system for many decades. It centers around the same objects: potentials, wave functions and transition operators, and, like calculations using $N N$ potential models, it treats the interaction of the nucleons non-perturbatively. The main difference to more traditional potential-model calculations of $N N$ system properties is the use of $\chi \mathrm{PT}$ to calculate the potentials and kernels that are the building blocks of the nuclear-physics calculation. Below we investigate this connection a little further, comparing and contrasting the $\chi \mathrm{PT}$ approach to the physics of the $N N$ system with physics based on more phenomenological potentials. Crucially though, the use of $\chi \mathrm{PT}$ to derive the basic building blocks that go into potential models allows for simplification and systematization of the calculations performed using the successful phenomenological potential models.

\subsection{The Weinberg Program: Power Counting}

The power counting for contributions to either the $N N$ potential, $V$, or the kernel for some process, $K_{\text {probe }}$, is just that of $\chi \mathrm{PT}$. In other words, we perform NDA on the kernels of processes, keeping track of factors of $p$ and $m_{\pi}$ in the case of $V$, and $p, m_{\pi}$, and $k$ - the probe energy/momentum - in the case of $K_{\text {probe. }}$. 
We generically denote all these "small" quantities as $P$. The expansion is then one in powers of $P /\left(\Lambda_{\chi}, M\right)$. A diagram which contributes to $V$ has an order $n$ in this expansion which is calculated by just multiplying together the powers of $P$ appearing in the diagram as follows:

- Each vertex from $\mathcal{L}^{(n)}$ counts as $P^{n}$;

- Each pion line counts as $P^{-2}$;

- Each loop counts as $P^{4}$;

- Each nucleon propagator counts as $P^{-1}$.

Note that these last two rules must be modified if we consider two-nucleonreducible diagrams as well, since in such diagrams the nucleon propagator can scale either as $P^{-1}$ or as $P^{-2}$, depending on whether the energy present is "small" or of order $m_{\pi}$.

For kernels $K_{\text {probe }}$ one additional rule must be added:

- Two-body contributions to $K_{\text {probe }}$ carry an extra factor of $P^{3}$.

This rule, which means that two-body contributions to the reaction mechanisms are systematically suppressed in this low-momentum regime, can be thought of as arising from the additional "loop" which occurs when a two-body reaction mechanism is sandwiched between $N N$ wave functions. Equivalently, it occurs because there is one less momentum-conserving delta function in a graph with a two-body contribution to $K_{\text {probe }}$. Either way the rule is again a straightforward consequence of NDA.

\subsection{Nucleon-Nucleon Potential}

The earliest discussion of constructing a $N N$ potentialin the context of Weinberg power counting was that by Weinberg in Refs. 18 , where $V^{(0)}$ was computed. Higher-order calculations were carried out by Ordónẽz et al., where energy-dependent pgtentials which represented the results for $V^{(0)}, V^{(2)}$, and $V^{(3)}$ were derived 129, 130.131. The energy-dependence of this potential was not intrinsic to $\chi P T$ and could be transformed away. In Ref. 132 Epelbaum et al. did this, using unitary transformations to turn these energy-dependent potentials into energy-independent ones [. By construction, this transformation leaves all S-matrix elements unaffected, and so there is nophysical difference between the potential of Ref. 132 and that of Refs. 129.130.131. The

${ }^{j}$ In Ref. 133 a similar connection between energy-dependent and energy-independent forms of the two-pion-exchange $N N$ force was established, thereby elyeiglating the difference tween old work on two-pion exchange by Brueckner and Watson 134 and Taketani et al. 135. 
isospin-violating $N N$ potential can be derived from $\chi \mathrm{PT}$ in an analogous fashion 136 . 137 , but we shall discuss only the charge-symmetric, charge-independent piece of the $N N$ interaction here.

The Lagrangian of $\chi P T$ is used to generate an $N N$ potential, $V$ as follows. The interaction $V^{(n)}$ contains all two-particle-irreducible $N N \rightarrow N N$ graphs that are of chiral order $n$ or lower. At leading order $(n=0)$, the chiral $N N$ potential has only two pieces

$$
V(\mathbf{q})=-\frac{g_{A}^{2}}{4 f_{\pi}^{2}} \frac{\sigma_{1} \cdot \mathbf{q} \sigma_{2} \cdot \mathbf{q}}{\mathbf{q}^{2}+m_{\pi}^{2}}\left(\vec{\tau}_{1} \cdot \vec{\tau}_{2}\right)+C_{S}+C_{T} \sigma_{1} \cdot \sigma_{2} .
$$

where $C_{S}$ and $C_{T}$ are coefficients of contact interactions that do not involve spatial gradients.

At any chiral order an analogous separation into a long- and short-distance $N N$ EFT potential can be made. Thus we will follow the authors of Ref. 138 and write $V=V_{\pi}+V_{\text {short }}$ At low orders in the expansion $V_{\text {short }}$ has a straightforward counting in powers of $P$, with the higher-derivative operators naively suppressed by powers of $P / \Lambda$.

Regardless of the scaling of the short-distance part, the long-distance piece is given by one-pion exchange plus irreducible two-pion exchange plus threepion exchange etc. The dimension of the contributions of these multi-pionexchange graphs produce in $V_{\pi}$ increases by two for each additional pion loop, as can be seen by simple $f_{\pi}$ counting. Consequently, irreducible multiple pion exchanges are suppressed by powers of $m_{\pi} / \Lambda$ and $p / \Lambda$. Having made a chiral expansion for both $V_{\text {short }}$ and $V_{\pi}$, we must truncate both at the same order in the chiral expansion parameter $\left(p, m_{\pi}\right) / \Lambda$.

The contributions to $V_{\text {short }}$ at zeroth and second-order in $\chi \mathrm{PT}$ are just 138:

$$
\begin{aligned}
& \left\langle\mathbf{p}^{\prime}\left|V_{\text {short }}^{(0)}\right| \mathbf{p}\right\rangle=C_{S}+C_{T} \sigma_{1} \cdot \sigma_{2} \\
& \left\langle\mathbf{p}^{\prime}\left|V_{\text {short }}^{(2)}\right| \mathbf{p}\right\rangle=\xi_{1} \mathbf{q}^{2}+\xi_{2} \mathbf{k}^{2}+\left(\xi_{3} \mathbf{q}^{2}+\xi_{4} \mathbf{k}^{2}\right)\left(\sigma_{1} \cdot \sigma_{2}\right) \\
& \quad+i \xi_{5} \frac{1}{2}\left(\sigma_{1}+\sigma_{2}\right) \cdot(\mathbf{q} \times \mathbf{k})+\xi_{6}\left(\sigma_{1} \cdot \mathbf{q}\right)\left(\sigma_{2} \cdot \mathbf{q}\right)+\xi_{7}\left(\sigma_{1} \cdot \mathbf{k}\right)\left(\sigma_{2} \cdot \mathbf{k}\right)
\end{aligned}
$$

where $\mathbf{k}=\mathbf{p}^{\prime}-\mathbf{p}$ and $\mathbf{k}=\left(\mathbf{p}^{\prime}+\mathbf{p}\right) / 2$. The translation into the short-range potential that is operative in a particular partial-wave is then a straightforward matter of taking matrix elements of these operators. We will not present the explict formulae here, but two points are immediately apparent:

1. This short-distance potential operates only in S-waves, P-waves, and to mix the ${ }^{3} S_{1}$ and ${ }^{3} D_{1}$ states.

2. In the ${ }^{1} S_{0}$ and ${ }^{3} S_{1}$ channels there is a LO and NLO contribution, with the NLO piece being proportional to $\left(p^{2}+p^{2}\right)$ by Hermiticity. 
3. In the next five partial waves $\left({ }^{1} P_{1},{ }^{3} P_{0},{ }^{3} P_{1},{ }^{3} P_{2}\right.$, and $\left.\epsilon_{1}\right)$ there is a straightforward set of linear equations which relates the combination of short-distance parameters that appears in any one partial wave to the parameters appearing in the potential.

This combination of facts means that by performing a fit with one parameter in the partial waves ${ }^{1} P_{1},{ }^{3} P_{0},{ }^{3} P_{1},{ }^{3} P_{2}$, and $\epsilon_{1}$, and two parameters in the partial waves ${ }^{1} S_{0}$ and ${ }^{3} S_{1}$ we may completely determine the short-distance potential.

At NLO the long-distance, pionic part of the potential is generated by the one-loop diagrams shown in Fig. 31. These diagrams are divergent in $\chi \mathrm{PT}$ and require renormalization, but the required renormalization may be made by absorbing the divergences in the constants $\xi_{1} \rightarrow \xi_{7}$ given above. Strictly speaking then, the relationship of the $\xi_{1} \rightarrow \xi_{7}$ which appear in Eq. (141) to the parameters which appear in the Lagrangian involves both the linear transformation mentioned above and this subtraction of certain forms arising in the calculation of the graphs shown in Fig. 31. After renormalization, the

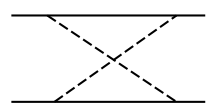

(a)

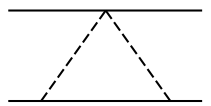

(c)

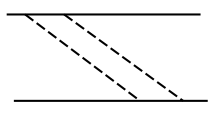

(b)

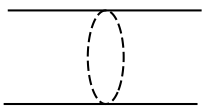

(d)

Figure 31: The two-pion-exchange graphs which are included in the $N N$ interaction at NLO.

long-distance part of $V_{\pi}$ at NLO is 131, 138

$$
\begin{gathered}
\left\langle\mathbf{p}^{\prime}\left|V_{\pi}^{(2)}\right| \mathbf{p}\right\rangle=-L(|\mathbf{q}|) \frac{3 g_{A}^{4}}{64 \pi^{2} f_{\pi}^{4}}\left[\left(\sigma_{1} \cdot \mathbf{q}\right)\left(\sigma_{2} \cdot \mathbf{q}\right)-\mathbf{q}^{2}\left(\sigma_{1} \cdot \sigma_{2}\right)\right] \\
-\frac{\vec{\tau}_{1} \cdot \vec{\tau}_{2}}{384 \pi^{2} f_{\pi}^{4}} L(|\mathbf{q}|)[ \\
4 m_{\pi}^{2}\left(5 g_{A}^{4}-4 g_{A}^{2}-1\right)+\mathbf{q}^{2}\left(23 g_{A}^{4}-10 g_{A}^{2}-1\right) \\
\left.+\frac{48 g_{A}^{4} m_{\pi}^{4}}{4 m_{\pi}^{2}+\mathbf{q}^{2}}\right],
\end{gathered}
$$


with

$$
L(|\mathbf{q}|)=\frac{1}{|\mathbf{q}|} \sqrt{4 m_{\pi}^{2}+\mathbf{q}^{2}} \log \left(\frac{\sqrt{4 m_{\pi}^{2}+\mathbf{q}^{2}}+|\mathbf{q}|}{2 m_{\pi}}\right) .
$$

At $\mathrm{N}^{2} \mathrm{LO}, O\left(P^{3} / \Lambda^{3}\right)$, there are no new short-distance operators. There are, however, new contributions to $V_{\pi}$ at this order. Where the WeinbergTomazawa term entered in the "triangle" and football graphs (diagrams (c) and (d) of Fig. 31) and contributed to $V^{(2)}$ we can now make one insertion of an operator from the pion-nucleon Lagrangian $\mathcal{L}_{\pi N}^{(2)}$ and generate contributions to $V_{\pi}^{(3)}$. After renormalization $V_{\pi}^{(3)}$ reads

$$
\begin{gathered}
\left\langle\mathbf{p}^{\prime}\left|V_{\pi}^{(3)}\right| \mathbf{p}\right\rangle=-\frac{3 g_{A}^{2}}{16 \pi f_{\pi}^{4}}\left[-\frac{g_{A}^{2} m_{\pi}^{5}}{16 M\left(4 m_{\pi}^{2}+\mathbf{q}^{2}\right)}\right. \\
\left.+\left(2 m_{\pi}^{2}\left(2 c_{1}-c_{3}\right)-\mathbf{q}^{2}\left(c_{3}+\frac{3 g_{A}^{2}}{16 M}\right)\right)\left(2 m_{\pi}^{2}+\mathbf{q}^{2}\right) A(|\mathbf{q}|)\right] \\
-\frac{g_{A}^{2}}{128 \pi M f_{\pi}^{4}}\left(\vec{\tau}_{1} \cdot \vec{\tau}_{2}\right)\left[-\frac{3 g_{A}^{2} m_{\pi}^{5}}{4 m_{\pi}^{2}+\mathbf{q}^{2}}\right. \\
\left.\quad+\left(4 m_{\pi}^{2}+2 \mathbf{q}^{2}-g_{A}^{2}\left(4 m_{\pi}^{2}+3 \mathbf{q}^{2}\right)\right)\left(2 m_{\pi}^{2}+\mathbf{q}^{2}\right) A(|\mathbf{q}|)\right] \\
+\frac{9 g_{A}^{4}}{512 \pi M f_{\pi}^{4}}\left(\left(\sigma_{1} \cdot \mathbf{q}\right)\left(\sigma_{2} \cdot \mathbf{q}\right)-\mathbf{q}^{2}\left(\sigma_{1} \cdot \sigma_{2}\right)\right)\left(2 m_{\pi}^{2}+\mathbf{q}^{2}\right) A(|\mathbf{q}|) \\
-\frac{g_{A}^{2}}{32 \pi f_{\pi}^{4}}\left(\vec{\tau}_{1} \cdot \vec{\tau}_{2}\right)\left(\left(\sigma_{1} \cdot \mathbf{q}\right)\left(\sigma_{2} \cdot \mathbf{q}\right)-\mathbf{q}^{2}\left(\sigma_{1} \cdot \sigma_{2}\right)\right) \\
\quad \times\left[\left(c_{4}+\frac{1}{4 M}\right)\left(4 m_{\pi}^{2}+\mathbf{q}^{2}\right)-\frac{g_{A}^{2}}{8 M}\left(10 m_{\pi}^{2}+3 \mathbf{q}^{2}\right)\right] A(|\mathbf{q}|) \\
-\quad \frac{3 g_{A}^{4}}{64 \pi M f_{\pi}^{4}} i\left(\sigma_{1}+\sigma_{2}\right) \cdot\left(\mathbf{p}{ }^{\prime} \times \mathbf{p}\right)\left(2 m_{\pi}^{2}+\mathbf{q}^{2}\right) A(|\mathbf{q}|) \\
-\frac{g_{A}^{2}\left(1-g_{A}^{2}\right)}{64 \pi M f_{\pi}^{4}}\left(\vec{\tau}_{1} \cdot \vec{\tau}_{2}\right) i\left(\sigma_{1}+\sigma_{2}\right) \cdot\left(\mathbf{p}^{\prime} \times \mathbf{p}\right)\left(4 m_{\pi}^{2}+\mathbf{q}^{2}\right) A(|\mathbf{q}|),
\end{gathered}
$$

with

$$
A(|\mathbf{q}|)=\frac{1}{2|\mathbf{q}|} \arctan \left(\frac{|\mathbf{q}|}{2 m_{\pi}}\right) .
$$

The free parameters $c_{1}, c_{3}$, and $c_{4}$ are related directly to data in the $\pi N$ system. The 's have been determined in recent fits to $\pi N$ scattering data in $\chi \mathrm{PT}$ 139,40. In principle then, there are no undetermined parameters in 
$V_{\pi}^{(3)}$. The fit to the $\pi N$ data extrapolated to the region inside the Mandelstam triangle is used by Epelbaum et al. to fix $c_{1}, c_{3}$, and $c_{4}$. Interestingly, Sugawara and Okubo wrote down a TPE potential which contained a large part of the correct TPE presented above as early as 1960 141. Stoks and Rijken also generated all of the two-pion exchange interactions written above 142 , although their potential also contained many other, more phenomenological, short-range contributions. Although the fact that $\chi \mathrm{PT}$ connects $\pi N$ data to $N N$ data in a model-indenendent way makes it similar in philosophy to these, and many other $143,144,145,146$, works, it was really only in Refs. 130,131 that a complete expression for the full chiral TPE was first written down.

This chiral TPE potential was also calculated by Kaiser et al. as part of a study we will discuss in more detail below 17 . Kaiser et al. observed that the combination $V^{(2)}+V^{(3)}$ provides strong scalar-isoscalar attraction. This attraction is governed by the LECs $c_{1}$ and $c_{3}$. This suggests that if two-pion exchange is calculated in a fashion consistent with chiral symmetry then there may be no need for the presence of an explicit $\sigma$-meson in the $N N$ potential.

This potential

$$
V^{[3]}=V^{(0)}+V_{\text {short }}^{(2)}+V_{\pi}^{(2)}+V_{\pi}^{(3)}
$$

must now be iterated in the Lippmann-Schwinger equation, Eq. (135). However, it is a poorly-behaved potential from a quantum mechanical point of view, since it is very singular at short distances, and the corresponding Hamiltonian is thus unbounded from below. This is perhaps not surprising, since we know that the short-distance physics in $\chi \mathrm{PT}$ is not the full short-distance physics of QCD. At distance scales $\sim 1 / \Lambda_{\chi}$ mechanisms not encoded in $\chi \mathrm{PT}$ will begin to play a dynamical role, and will regulate the behavior of the singular potential $V^{[3]}$. In order to calculate with the potential Refs. 131.138 introduced cutoffs on the potential before iteration, defining a regulated potential

$$
\left\langle\mathbf{p}^{\prime}\left|V_{R}\right| \mathbf{p}\right\rangle=f_{\Lambda}\left(\left|\mathbf{p}^{\prime}\right|\right)\left\langle\mathbf{p}^{\prime}|V| \mathbf{p}\right\rangle f_{\Lambda}\left(\left|\mathbf{p}^{\prime}\right|\right)
$$

where $f_{\Lambda}(|\mathbf{p}|)$ is some regulator function that goes to zero sufficiently fast as $|\mathbf{p}|$ goes to infinity, and obeys $f_{\Lambda}(0)=1$, e.g.,

$$
f_{\Lambda}(|\mathbf{p}|)=\exp \left(-|\mathbf{p}|^{4} / \Lambda^{4}\right)
$$

This regulates the potential. Practical implementation of renormalization is then quite simple. Whe regulator mass, $\Lambda$, fixed, one tunes the shortdistance coefficients $10,147,24,116$ so as to reproduce some specified low-energy observables. 
With renormalizable theories, the next step would be to take the regulator to infinity. Here $\Lambda$ should be of the order of the high energy scale $\Lambda_{\chi}$. There are two reasons for this. Firstly, NDA applies when $\Lambda \sim \Lambda_{\chi}$. Secondly, in the Weinberg scheme subleading terms are resummed to all orders and may become large for large $\Lambda$ as the counterterms required to renormalize these contributions are not present.

The algorithm for generating predictions from the regulated third-order chiral potential $V_{R}^{(3)}$ adopted in Ref. 138 is then:

1. Choose a regulator mass $\Lambda$.

2. Fit the constants $C_{S}, C_{T}$, and $\xi_{1}-\xi_{7}$ to the $\mathrm{S}$ and $\mathrm{P}$-wave phase shifts and $\epsilon_{1}$ from the Nijmegen PSA 2 up to $T_{\text {lab }}=100 \mathrm{MeV}$.

3. Predict these same phases from $T_{\text {lab }}=100 \mathrm{MeV}$ to $T_{\text {lab }}=300 \mathrm{MeV}$, and predict other phase-shifts over the full energy range.
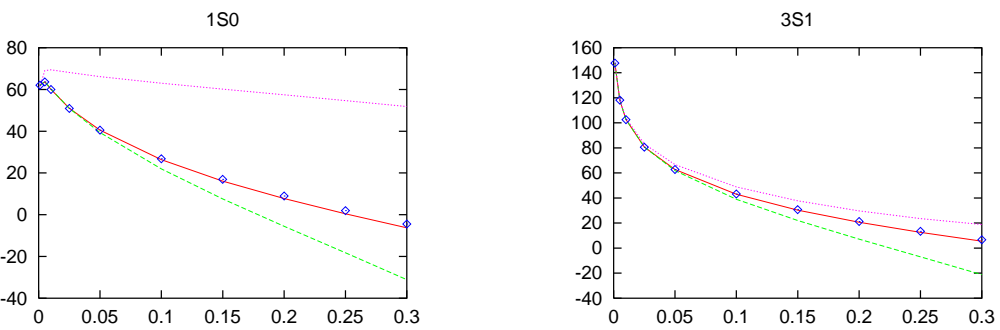

Figure 32: Predictions for the ${ }^{1} S_{0}$ and ${ }^{3} S_{1}$ phase shifts from Ref. 138. The dotted, dashed, and solid curves represent the results from the LO, NLO, and $\mathrm{N}^{2} \mathrm{LO}$ potential. At NLO and $\mathrm{N}^{2} \mathrm{LO}$ the two parameters in this channel are fitted to the phase shifts below $T_{\text {lab }}=100 \mathrm{MeV}$. The squares represent the Nijmegen PSA phases. The exponential regulator was employed with $\Lambda=500 \mathrm{MeV}$ at NLO, and $\Lambda=875 \mathrm{MeV}$ at $\mathrm{N}^{2} \mathrm{LO}$.

In general the results for the $S$ and $P$ waves (where at least one fit parameter was present in each case) are quite good, although the behavior of the ${ }^{3} P_{2}$ phase shift causes some concern. Some of these results are displayed in Figs. 32 and 33 .

We stress once again that there are no free parameters to fit in partialwaves with $L \geq 2$. Thus, the phase shifts calculated there are parameter-free predictions of this approach. In the $D$-waves, the results are in quite good agreement with the Nijmegen PSA although some deviation begins to be seen around $T_{\text {lab }}=200 \mathrm{MeV}$ (see Fig. 35). Epelbaum et al. also note that these 

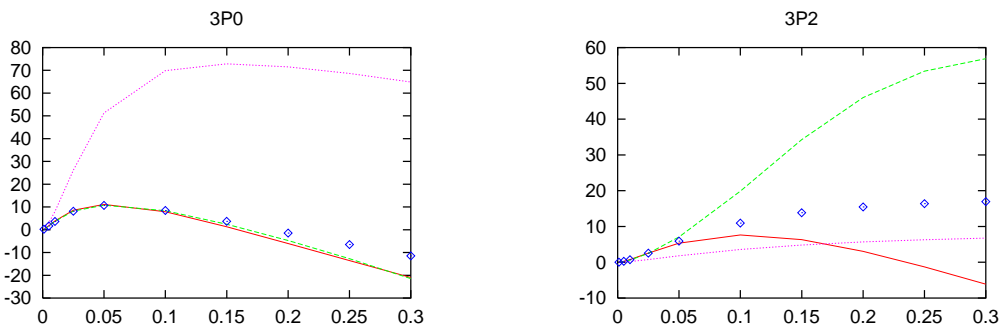

Figure 33: The ${ }^{3} P_{0}$ and ${ }^{3} P_{2}$ phase shifts as calculated in Ref. 138. In this case there is one parameter at $\mathrm{NLO}$ and $\mathrm{N}^{2} \mathrm{LO}$, which is fitted to the phase shifts below $T_{\text {lab }}=100 \mathrm{MeV}$. Legend as in Fig. 32.
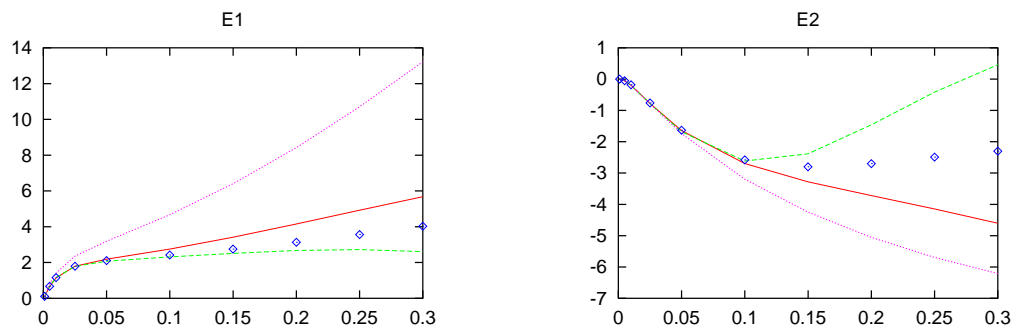

Figure 34: The mixing parameters $\epsilon_{1}$ and $\epsilon_{2}$ from Ref. 33 . There is one fit parameter in $\epsilon_{1}$. The results for $T_{\mathrm{lab}} \geq 100 \mathrm{MeV}$ in $\epsilon_{1}$, as well as allof the results for $\epsilon_{2}$ are predictions. Legend as in Fig. 32 . 
phases are quite sensitive to the value of the cutoff chosen. This may be because at next order, in $V^{(4)}$, contact interactions enter which will affect the $D$-wave $N N$ channels. Meanwhile, the mixing parameter $\epsilon_{2}$ is not well reproduced above $T_{\text {lab }}=100 \mathrm{MeV}$, while the results for the triplet $F$-waves are really quite poor. It seems that for these smaller $N N$ phase shifts a higher-order calculation is needed for an accurate description.
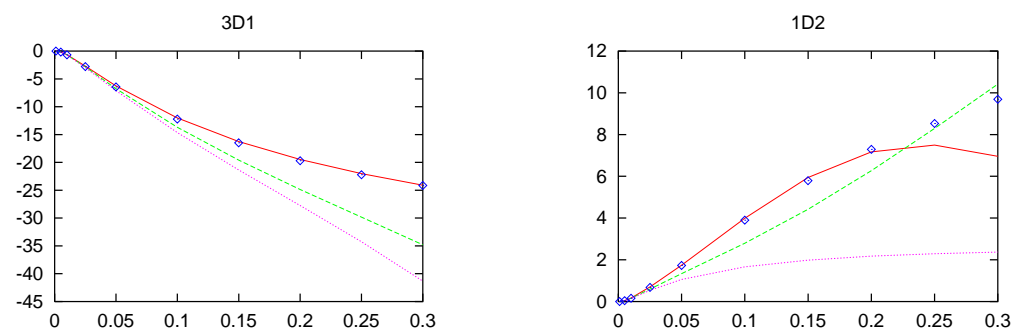

Figure 35: The ${ }^{3} D_{1}$ and ${ }^{1} D_{2}$ phases from Ref.138. In this case there are no free parameters. Legend as in Fig. 32 .

As the orbital angular momentum is increased the Born approximation works increasingly well, and there is, in fact, little need to iterate the potential. In this way the study of Epelbaum et al. reduces to that of Ref. 17 in the higher partial-waves. In that work Kaiser et al. calculated the following perturbative $N N$ amplitude

$$
T_{\text {pert }}=V_{\pi}^{(0)}+V_{\pi}^{(0)} G_{0} V_{\pi}^{(0)}+V_{\pi}^{(2)}+V_{\pi}^{(3)} .
$$

In other words, these authors employed the potentials $V_{\pi}^{(2)}$ and $V_{\pi}^{(3)}$ at tree level, and OPEP in second Born approximation (i.e. iterated once) as the $N N$ amplitude. The graphs included are those of Figs. 30 and 31 together with the triangle and football graphs with insertions from $\mathcal{L}_{\pi N}^{(2)}$. This is an approximation to the full $N N$ t-matrix obtained by iterating the chiral $N N$ potential $V^{(3)}$. It works increasingly well as the angular momentum of the $N N$ state increases, since the centrifugal barrier ensures that only the long-range, weak, part of the $N N$ force is probed in these waves. Comparison of the results of Refs. 17 and 138 indicates that iteration begins to be important below $L=4$, but that for $L \geq 4$ the amplitude (148) does a good job of reproducing the phase shifts.

Indeed, in these peripheral partial waves one-pion exchange alone, i.e. the interaction $V_{\pi}^{(0)}$ uniterated, reproduces the experimental data reasonably well-as has been known for many years 148 . For $L=6$ the effects of the parts 
Table 2: Results for the PWA with different long-range interactions and $R=1.4 \mathrm{fm}$.

\begin{tabular}{l|cc} 
& \#Parameters & $\chi_{\min }^{2}$ \\
\hline $\mathrm{OPE}$ & 31 & 2026.2 \\
$\mathrm{OPE}+V^{(2)}$ & 28 & 1984.7 \\
$\mathrm{OPE}+V^{(2)}+V^{(3)}$ & 23 & 1934.5
\end{tabular}

of $T_{\text {pert }}$ other than $V_{\pi}^{(0)}$ are really quite difficult to see below $T_{\text {lab }}=200 \mathrm{MeV}$. However, for the $G$ and $H$-waves the inclusion of $V^{(2)}$ and $V^{(3)}$ improves the agreement with the data.

A calculation of the next-order piece of the chiral $N N$ potential, $V^{(4)}$ will bring two new ingredients. Firstly, a number of short-distance operators will arise. The role that these might play in the ${ }^{1} S_{0}$ channel has been considered in Ref. 149. In general though, a number of channels will have to be considered: these operators will have to be fitted to the $S, P$, and $D$ wave phase shifts, as well as to mixing parameters. Secondly, many two-loop contributions to the potential - some of which have been calculated in Refs. 150.151 - will need to be added to the interaction. The calculation of the full $V^{(4)}$ is a Herculean task. Nevertheless, we can get some feeling for the size of higher-order effects by including more freedom in the short-distance part of the interaction.

This is essentially the direction taken in a recent calculation by the Nijmegen group 152. Rentmeester et al. calculated the piece of the chiral potential $V^{(3)}$ of Eq. (145) which acts at radii $r>1 / \Lambda_{\chi}$-i.e. the potential's long-range tail - and incorporated it into the Nijmegen PSA machinery. Schematically, they worked with a potential

$$
V(r)=V_{\text {short }}(r) \theta(R-r)+\left[\sum_{i=0}^{3} V_{\pi}^{(i)}+V_{\mathrm{em}}(r)\right] \theta(r-R),
$$

where $V_{\mathrm{em}}$ accounts for electromagnetic corrections to the long-distance part of the potential. As for the short-distance part, $V_{\text {short }}$ is only operative at radii $r<R$, and it depends on many free parameters. These parameters are tuned so as to reproduce the experimental phase shifts. Fixing the boundary $R$ at $1.4 \mathrm{fm}$, and the constant $c_{1}=-0.76 \mathrm{GeV}^{-1}$, Rentmeester et al. tuned 23 parameters in $V_{\text {short }}$ and the $\pi N$ LECs $c_{3}$ and $c_{4}$ to fit $1951 \mathrm{pp}$ scattering data below $T_{\text {lab }}=350 \mathrm{MeV}$. The resultant minimum $\chi^{2}$ is very close to the theoretical ideal of one per degree of freedom (see Table 2).

This is a strong indication that the chiral TPE is the intermediate-range part of the $N N$ force. This conclusion is reinforced by what happens if the chiral TPE is removed from the interaction. In that case the minimum $\chi^{2}$ 
shoots up appreciably, even if a number of new parameters are introduced in the short-distance interaction. The final indication that the chiral $N N$ potential is reproducing the physics of the $N N$ system in this phase-shift analysis is that the values of $c_{3}$ and $c_{4}$ obtained from this fit are $c_{3}=-4.99 \pm 0.21 \mathrm{GeV}^{-1}$ and $c_{4}=5.62 \pm 0.59 \mathrm{GeV}^{-1}$. These, together with the value of $c_{1}$, agree with the values extracted for these LECs from the $\pi N$ data, within the significant uncertainties associated with fitting in the $\pi N$ system.

\section{External Probes of the Deuteron}

In scattering processes involving the deuteron EFT has proved more successful phenomenologically than traditional potential models. Part of this success is due to the fact that the relation between exchange currents and potentials is dictated by the rules of field theory and is therefore unambiguous in EFT. Perhaps more importantly, EFT relates scattering processes involving the nucleon alone to nuclear scattering processes in a controlled manner; all of the phenomenology of $\chi P T$ in the single nucleon sector makes its appearance at some level in the nuclear sector. Hence, counterterms which are difficult to measure in experiments with nucleons can sometimes be extracted in a systematic way from nuclear experiments. This is particularly important given the absence of suitable neutron targets. Weinberg power-counting has led to fruitful computation of many pionic and photonic probes of the two-nucleon system 153 , including electron-deuteron scattering, electromagnetic deuteron form factors, neutral pion photoproduction on the deuteron at threshold 154 L55, neutral pion electroprofuction on the deuteron at threshold 156 , the pion-deuteron scattering length9.157, Compton scattering on the deuteron 158 , as well as $p n$ radiative capture 63 64 and the solar burning process $p p \rightarrow d e^{+} \nu 159$. We will consider each of these processes in turn.

On the technical side, it should be noted that typical nucleon momenta inside the deuteron are on the order of $m_{\pi}$, and consequently, we expect no convergence problems in the $\chi P T$ expansion of any low-momentum electromagnetic or pionic probe of the deuteron. We will use wavefunctions generated using modern nucleon-nucleon potentials as well as wavefunctions recently computed in $\chi P T$. Generally we find that any wavefunction with the correct binding energy gives equivalent results to within the theoretical error expected from neglected higher orders in the chiral expansion. 


\subsection{Electron-Deuteron Scattering}

For simplicity we will work in the Breit frame in calculating matrix elements of the form,

$$
\left\langle j \mathbf{q} / 2\left|J_{\mathrm{em}}^{\mu}\right| i-\mathbf{q} / 2\right\rangle
$$

where a virtual photon of three-momentum $\mathbf{q}=q \hat{z}$ couples to a deuteron electromagnetic current when the deuteron is in states with specific magnetic quantum numbers $i$ and $j$, as discussed earlier. The electromagnetic structure of the deuteron may be parametrized in terms of the three form factors, $F_{C}\left(q^{2}\right)$, $F_{M}\left(q^{2}\right)$, and $F_{Q}\left(q^{2}\right)$ defined in Eq. (52).

\subsection{The deuteron current}

In general, the current to which the electron couples in electron-deuteron scattering may be expressed as:

$$
\left\langle\mathbf{P}^{\prime} \mathbf{p}^{\prime}\left|J_{\mu}\right| \mathbf{P} \mathbf{p}\right\rangle=(2 \pi)^{3} j_{\mu}^{(1)}(\mathbf{p}, \mathbf{q}) \delta\left(\mathbf{p}^{\prime}-\mathbf{p}-\mathbf{q} / 2\right)+j_{\mu}^{(2)}\left(\mathbf{p}, \mathbf{p}^{\prime} ; \mathbf{q}\right),
$$

where $\mathbf{p}$ and $\mathbf{p}^{\prime}$ are the initial and final relative momenta in the deuteron state, and $\mathbf{q}=\mathbf{P}^{\prime}-\mathbf{P}$ is the three-momentum of the photon exchanged between the electron and the deuteron. The one- and two-body currents discussed here are depicted in Fig. 36. This is a useful decomposition because Weinberg power-counting predicts that the two-body currents are generically suppressed relative to one-body currents.
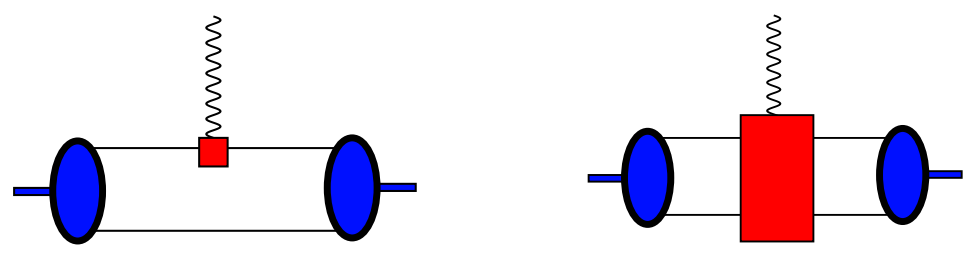

Figure 36: One- and two-body current contributions to electron-deuteron scattering. The square and rectangle indicate, respectively a $\gamma N N$ vertex, and the two-body $N N \rightarrow N N \gamma$ kernel. The blobs are deuteron vertex functions.

For on-shell nucleons the most general form that the single-nucleon current, $j_{\mu}^{(1)}$, can take, consistent with Lorentz invariance, time-reversal invariance, and current conservation, is:

$$
j_{\mu}^{(1)}(\mathbf{p}, \mathbf{q})=e_{N} \bar{u}(\mathbf{p}+3 \mathbf{q} / 4)\left[F_{1}\left(q^{2}\right) \gamma_{\mu}+F_{2}\left(q^{2}\right) \frac{i}{2 M} \sigma_{\mu \nu} Q^{\nu}\right] u(\mathbf{p}-\mathbf{q} / 4),
$$


where $e_{N}$ is the nucleonic charge. For the deuteron case, $F_{1}$ and $F_{2}$ are isoscalar combinations of the proton and neutron Dirac and Pauli form factors $(i=1,2)$

$$
F_{i}\left(q^{2}\right)=F_{i}^{(p)}\left(q^{2}\right)+F_{i}^{(n)}\left(q^{2}\right) .
$$

It is straightforward to calculate these isoscalar Dirac and Pauli form factors in $\chi \mathrm{PT}$ 160161.162. At LO the only contribution comes from $\mathcal{L}_{\pi N}^{(1)}$, and corresponds to an $A_{0}$ photon coupling to the charge of the nucleon. At NLO the current $j_{+}$receives contributions from the E1 and M1 vertices of $\mathcal{L}_{\pi N}^{(2)}$. Thus the first two orders of the calculation are trivial, and lead to the currents:

$$
j_{0}^{(1)}(\mathbf{p}, \mathbf{q})=e, j_{+}^{(1)}(\mathbf{p}, \mathbf{q})=e\left(\frac{p_{+}}{M}+\frac{q}{2 M}\left(\kappa_{p}+\kappa_{n}+1\right) \sigma_{+}\right) .
$$

Corrections at next order will include relativistic effects, and the effects of nucleon structure. The former are suppressed by ratios of momenta to the nucleon mass, which we denote generically by the mnemonic: $\delta^{2} \sim p^{2} / M^{2}, p$. $Q / M^{2}, q^{2} / M^{2}$. Corrections at order $\delta^{2}$ include the usual spin-orbit and DiracFoldy relativistic corrections to the charge operator 163 . Meanwhile, the second expansion will be governed by the parameter $q^{2} / \Lambda_{\chi}^{2}$. In the isoscalar form factors these structure corrections are purely due to short-distance counterterms, since a photon cannot couple to two pions in an isoscalar state.

We now turn to the task of quantifying the effect of two-body currents on the observables under consideration. The power counting here was first explained in Ref.63 and has beep further worked out for the exchange currents which contribute to $F_{M}$ in Ref. 164 .

The contribution of two two-body pionic mechanisms to the deuteronic current is depicted in Fig. 37. It is straightforward to count the powers of momentum or pion mass in such graphs. These current contributions scale at least as $P^{3}$. Therefore we expect that such meson-exchange currents will be suppressed by $P^{2} / \Lambda_{\chi}^{2}$ relative to the lowest-order $\gamma N N$ interaction, which scales as $P$.

Since $j_{+}$begins only at $O\left(P^{2}\right)$ it might seem that meson-exchange currents are only suppressed by one power of $P$ in the deuteron form factor $F_{M}$, but this is not the case. In fact, although the leading effect of meson-exchange currents is nominally $O\left(P^{3}\right)$, the meson-exchange current which occurs at that order is isovector in character and thus vanishes in the deuteron. It is well known that this $O\left(P^{3}\right)$ isovector current is the leading MEC correction to processes such as electrodisintegration of the deuteron, and $\gamma d \rightarrow n p$ (see, for instance,

${ }^{k}$ Here we are counting the charge $e$ as of order $P$ in order to simplify matters. 


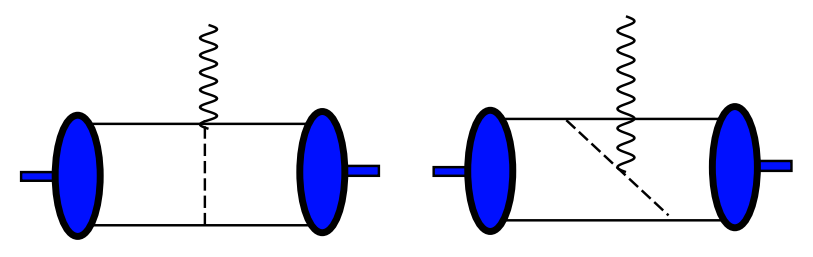

Figure 37: The contribution to ed scattering from the LO pionic current. Here all vertices are from the LO chiral perturbation theory Lagrangian. The graphs with nucleons one and two interchanged also contribute.

Ref. 165, 62). In practice this results in the leading effect from meson-exchange currents coming at $O\left(P^{4}\right)$, or $\mathrm{N}^{2} \mathrm{LO}$ in the deuteron form factors. In such an $O\left(P^{4}\right)$ exchange current the $\gamma \pi$ contact interaction with the nucleon has a coefficient that is related to known constants by the requirements of gauge invariance and reparametrization invariance. We note that this correction to the charge operator is phenomenologically important in potential-model calculations of $F_{C}$, 166 .

At some order above $O\left(P^{3}\right)$ operators enter whose coefficient is not constrained by symmetries, but instead is sensitive to short-distance physics. These have only a small effect in $F_{C}$ and $F_{M}$ for $q \lesssim 1 \mathrm{GeV}$, but they may affect $F_{Q}$ markedly, since the natural size of $F_{Q}$ at $q=0$ is much smaller than that of $F_{C}$ and $F_{M}$. A natural expectation for $\left.F_{Q}\right|_{q=0} \equiv Q_{d}$ is:

$$
Q_{d} \sim \frac{\eta_{s d}}{\gamma^{2}}
$$

Here $\eta_{s d}$ - the asymptotic D-to-S normalization-enters because an S-wave deuteron cannot emit a quadrupole photon via any one-body current mechanism. Thus the power-counting for the contribution of one-body currents to $F_{Q}$ is exactly the same as for similar contributions to $F_{C}$. However, two-body currents can connect the S-wave pieces of the deuteron initial- and final-state wave functions one to another and still result in the emission of a quadrupole photon 39. The lowest-order Lagrangian which can generate this effect is that written down in Eq. (56). This two-body operator does not enter in the expansion of the deuteron current until $O\left(P^{5}\right)$ in Weinberg counting. On the other hand, crucially it contains no powers of $\eta_{s d}$. Hence, numerically it can be important at much lower order than naively expected. Counting only overall powers of $p, M$, and $\Lambda$, we see that the contribution of this operator to $Q_{d}$ will be of order 


$$
\Delta Q_{d} \sim \frac{\left\langle p^{3}\right\rangle}{M \Lambda^{2}} \frac{1}{\Lambda^{2}}
$$

Although this is formally of order $P^{5}$, numerically it is as important as $\mathrm{N}^{2} \mathrm{LO}$ corrections to $Q 167$

From Ref. 131138 we have both NLO and $\mathrm{N}^{2} \mathrm{LO} N N$ potentials, derived from chiral perturbation theory. The Schrödinger equation, Eq. (138), can be solved with these potentials to generate deuteron wave functions. We can then use the systematic chiral expansion of the deuteron electromagnetic current to calculate the electromagnetic form factors of the deuteron. These results are compared with results from a sophisticated potential model, the Nijm93 potential. In both cases we employ the nonrelativistic impulse approximation with point nucleons, since this represents a calculation to NLO in the ed scattering kernel. Before calculating $F_{C}, F_{Q}$, and $F_{M}$ though, we will spend some time discussing results for static properties of the deuteron.

\subsection{Static Moments}

The values of the form factors $F_{M}$ and $F_{Q}$ at $q^{2}=0$ are related to the magnetic and quadrupole moments of the deuteron via the definitions in Eq. (53). If no meson-exchange currents (i.e. no local four-nucleon-one-photon operators) enter the ed scattering kernel, and there are no relativistic corrections to the current, then $\mu_{d}$ and $Q_{d}$ are given by simple integrals of the deuteron radial wave functions $u(r)$ (S-wave) and $w(r)$ (D-wave),

$$
\begin{aligned}
\mu_{d} & =\kappa_{p}+\kappa_{n}-\frac{3}{2}\left(\kappa_{p}+\kappa_{n}-\frac{1}{2}\right) \int_{0}^{\infty} d r w^{2}(r) \\
\mu_{\mathcal{Q}} & =\frac{1}{\sqrt{50}} \int_{0}^{\infty} d r r^{2} u(r) w(r)-\frac{1}{20} \int_{0}^{\infty} d r r^{2} w^{2}(r) .
\end{aligned}
$$

Another interesting static property of the deuteron is its matter radius, $r_{d}$. This is dominated by the long-distance part of the deuteron wave function, since it occurs in an integral weighted by $r^{2}$ :

$$
r_{d}^{2}=\int_{0}^{\infty} d r r^{2}\left[u^{2}(r)+w^{2}(r)\right]
$$

Employing the wave functions derived above, along with three leading potential models 4 国 leads to the results shown in Table 3. The experimental data is included for comparison only, since we have used a very simple electromagnetic current to generate these results. 


\begin{tabular}{|c|c|c|c|}
\hline & $r_{d}(\mathrm{fm})$ & $\mu_{d}\left(\mu_{N}\right)$ & $\mu_{\mathcal{Q}}\left(\mathrm{fm}^{2}\right)$ \\
\hline \hline Nijm93 & 1.968 & 0.847 & 0.271 \\
\hline AV18 & 1.967 & 0.847 & 0.270 \\
\hline CD-Bonn & 1.966 & 0.852 & 0.270 \\
\hline$\chi$ PT NLO & 1.975 & 0.859 & 0.266 \\
\hline$\chi$ PT N ${ }^{2} \mathrm{LO}$ & 1.967 & 0.845 & 0.262 \\
\hline Experiment & $1.971(6)$ & $0.857406(1)$ & $0.2859(3)$ \\
\hline
\end{tabular}

Table 3: Static moments of the deuteron for the Nijm93, CD-Bonn, and AY18 potentials, as well as for the NLO and $\mathrm{N}^{2} \mathrm{LO} \chi \mathrm{PT}$ potentials of Ref. 138.

The table shows that the deuteron matter radius is the same to three significant figures in all six calculations. Moreover, the results are all within the error bar of the value extracted from hydrogen-deuterium isostope-shift measurements 168, 169. For the magnetic moment the situation is not quite so good, but it is clear that $\mu_{d}$ is predominantly determined by $\kappa_{p}$ and $\kappa_{n}$. The size of the spread in the theoretical models - and of their discrepancy with the experimental value 454 - is consistent with the magnitude expected for higher-order contributions to the deuteron current.

Park et al. 59 and Phillips and Cohen 167 have also performed calculations of the static properties of the deuteron in EFT-motivated approaches. Both of these papers employed one-pion exchange as the long distance part of the $N N$ potential - rather than chiral two-pion exchange - and various different short-distance regulators. The results are in accord with the above findings. Firstly, $r_{d}$ is extremely well-predicted in the effective theory, once $A_{S}$, the asymptotic S-state normalization of the deuteron wave function, and $B$, the deuteron binding energy, are reproduced. This is in line with the findings of $\operatorname{EFT}(\AA)$ and ERT. The deuteron radius is largely determined by the tail of the deuteron wave function. Similarly, $\mu_{d}$ is largely insensitive to the shortdistance physics, since it is dominated by $\kappa_{p}$ and $\kappa_{n}$.

The large variation in $Q_{d}$ between the calculations presented in Table 3 was also seen in the studies of Ref. 159 and 167. There is considerable sensitivity to the short-distance physics in this observable. Presumably, this is why the five theoretical values all differ markedly from the experimental number. Apparently there are significant contributions to this quantity from physics beyond those of single nucleon operators. One such piece of physics is a two-body current resulting in the emission of a quadrupole photon 39 . As argued above, a natural size for the contribution of this operator to $\mu_{\mathcal{Q}}$, will give approximately as large an effect as terms one order in the expansion beyond what we have 
considered here.

\subsection{Electromagnetic Deuteron Form Factors}

$F_{C}$ calculated with the NLO wave function of $\chi \mathrm{PT}$ is shown in Fig. 38. It is compared with results from the Nijm93 wave function. The agreement is good for photon momenta of up to about $500 \mathrm{MeV}$ for a regulator mass $\Lambda=600 \mathrm{MeV}$ in the exponential of Eq. (147).

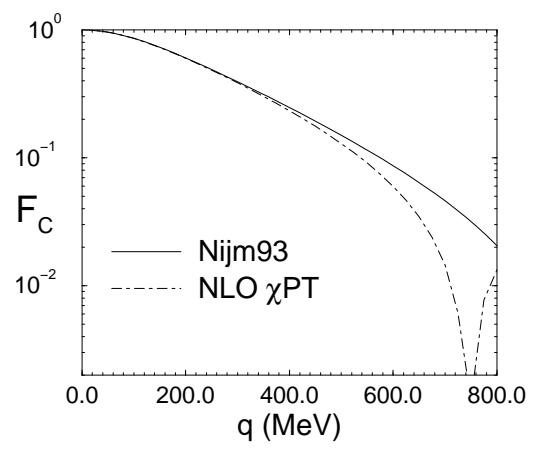

Figure 38: The charge form factor of the deuteron at NLO. The solid line is the result for the Nijm93 potential, while the dot-dashed line is a calculation with the NLO chiral potential.

Results are presented for the deuteron magnetic form factor $F_{M}$ in Fig. 39 . These plots lead to conclusions similar to those inferred from the plot of $F_{C}$. This is a consequence of the dominance of the S-wave pieces of the wave function in determining the magnetic structure of the deuteron at low $q$.

The situation is somewhat different for the quadrupole form factor $F_{Q}$. Since the quadrupole moments obtained in the Nijm93 and NLO $\chi \mathrm{PT}$ calculations do not quite agree there is a discrepancy of a few percent between the two form factors at $q=0$. On the other hand, the shape of the two curves is essentially the same out to $q \approx 500 \mathrm{MeV}$. In fact, the quadrupole moments in both calculations differ appreciably from the experimental result. Simple power-counting arguments suggest that a two-body counterterm might be important in $F_{Q}$. To further examinethis problem, we have calculated the ed tensor-polarization observable $T_{20}$ in four different ways. We have performed two different types of calculation for each of the two wave functions: Nijm93 and NLO $\chi \mathrm{PT}$ :

- A calculation with single nucleon operators; 

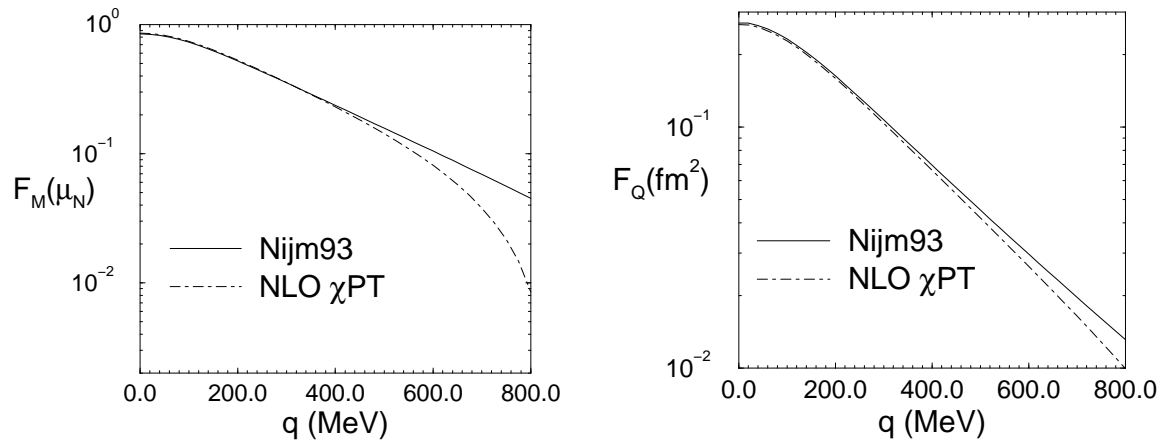

Figure 39: The magnetic and quadrupole form factors of the deuteron for the Nijm93 and NLO $\chi$ PT wave functions. Legend as in Fig. 38 .

- A calculation where we add - in an admittedly non-systematic way-a two-body counterterm which shifts the value of the quadrupole moment in both calculations to the experimental value.

The results of this investigation are presented in Fig. 401 We see that when the same currents are used the results for $T_{20}$ are the same with both wave functions out to $q \approx 500 \mathrm{MeV}$. This is just a restatement of the results presented for electromagnetic form factors above. However, we also see that shifting the value of $Q_{d}$ from the impulse approximation result $Q_{d}=0.27 \mathrm{fm}^{2}$ to the experimental value $Q_{d}=0.29 \mathrm{fm}^{2}$, has a marked effect on $T_{20}$ at momentum transfers of order a few hundred $\mathrm{MeV}$. Moreover, it would seem that once the deuteron quadrupole moment is reproduced hoth the petential model and the EFT calculation describe the data of Refs. 179, 171. 172, $173,174,175$ fairly well out to momenta $q \approx 600 \mathrm{MeV}$. This suggests that the physics which determines $T_{20}$ at these momentum transfers is the physics of one-pion-exchange - which the two potentials here have in common - along with a deuteron which has the right binding energy and the correct quadrupole moment.

\section{$8.5 \gamma d \rightarrow \pi^{0} d$}

Pion photoproduction on the nucleon near threshold has been studied up to $O\left(P^{4}\right)$ in $\chi P T$ with the delta integrated out in Ref. 176. The differential crosssection for a photon of momentum $k$ to produce a pion of momentum $q$ at threshold is pure $S$ wave and given by

\footnotetext{
${ }^{l}$ Thanks to Matt Dorsten for his help in generating this figure.
} 


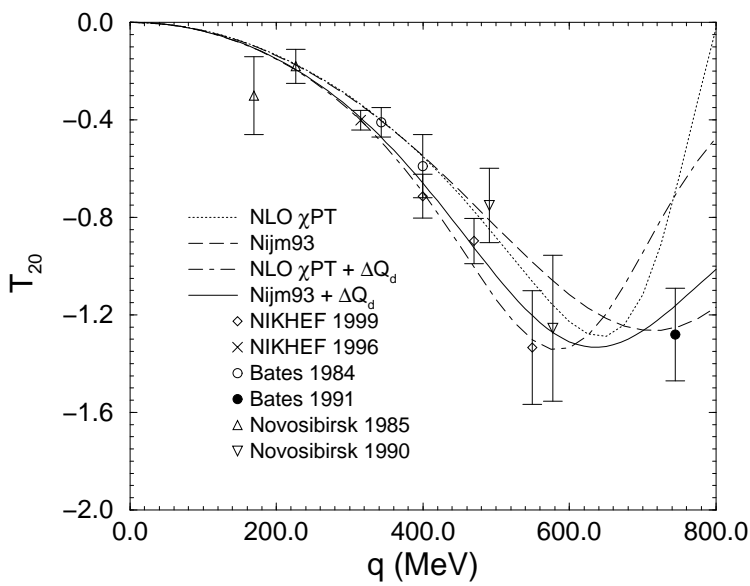

Figure 40: The ed scattering tensor polarization observable $T_{20}$. The dashed (dotted) lines correspond to insertions of a single nucleon operator only, using the Nijm93 (NLO $\chi \mathrm{PT}$ ) wave function. The solid (dot-dashed) line is the Nijm93 (NLO $\chi \mathrm{PT}$ ) result when a two-body counterterm is added so therimental data is taken from Refs. 170.171 .172 .173 .174 .175$.

$$
\left.\frac{|\vec{k}|}{|\vec{q}|} \frac{d \sigma}{d \Omega}\right|_{|\vec{q}|=0}=\left|E_{0+}\right|^{2}
$$

where $E_{0+}$ is the electric dipole amplitude. At LO, $O(P)$, there are contributions only to charged pion channels arising from minimal substitution on the pion-nucleon vertex ("Kroll-Ruderman term") and from the pion pole. Therefore the charged channels are expected to be large compared to the neutral channels. At $O\left(P^{2}\right)$, minimal substitution on the relativistic correction to the pion-nucleon vertex contributes to photoproduction on the proton. At $O\left(P^{3}\right)$ there are tree-level contributions involving the magnetic moment of the nucleon. Together with the previous orders, this reproduces an old "low-energy theorem". However, at $O\left(P^{3}\right)$ there are also finite loop corrections where the photon interacts with a virtual pion which then rescatters on the nucleon. This large quantum effect was missed in the old "low-energy theorem" and in most models. At $O\left(P^{4}\right)$ there are loops with relativistic corrections at the vertices together with new counterterms. Results 176 for $E_{0+}$ up to $O\left(P^{4}\right)$ from a fit to data above threshold constrained by resonance saturation of parameters are 
shown in Table 月同.

Table 4: Values for $E_{0+}$ in units of $10^{-3} / m_{\pi^{+}}$at various orders in $\chi P T$ and in experiments, for the different channels.

\begin{tabular}{lccccc}
\hline$E_{0+}\left(10^{-3} / m_{\pi^{+}}\right)$ & $P$ & $P^{2}$ & $P^{3}$ & $P^{4}$ & Experiment \\
\hline$\gamma p \rightarrow \pi^{+} n$ & 34.0 & 26.4 & 28.9 & 28.2 & $27.9 \pm 0.5$ \\
& & & & $28.8 \pm 0.7$ \\
$\gamma n \rightarrow \pi^{-} p$ & -34.0 & -31.5 & -32.9 & -32.7 & $-31.4 \pm 1.3$ \\
& & & & $-32.2 \pm 1.2$ \\
& \multirow{2}{*}{0} & -3.58 & 0.96 & -1.16 & $-1.31 \pm 0.08$ \\
$\gamma p \rightarrow \pi^{0} p$ & 0 & 0 & & & $-1.32 \pm 0.08$ \\
$\gamma n \rightarrow \pi^{0} n$ & 0 & & & & \\
\hline
\end{tabular}

Charged-pion channels are indeed larger than neutral pion channels and show good convergence and agreement with experimental data. For neutralpion channels convergence is less apparent, but the absolute values are much smaller. The $O\left(P^{4}\right)$ result for $\gamma p \rightarrow \pi^{0} p$ is in relatively good agreement with the recent results from Mainz and Saskatoon. To the same order there is a prediction for the near-threshold behavior of the $\gamma n \rightarrow \pi^{0} n$ reaction; the cross-section is considerably larger than that obtained in models that omit the important one-loop $O\left(P^{3}\right)$ diagrams. In fact, the neutron cross-section is a factor of four larger than the proton cross-section, in violation with classical intuition. Neutral pion photoproduction on the deuteron is the best candidate for a test of this peculiar prediction for the neutron.

We now consider pion photoproduction on the deuteron 155 . Consider a process with two nucleons, a single photon and a single pion in the initial and final state, respectively. Following the counting rules outlined above, the contributions are ordered as follows: $O(P)$ : At LO, the matrix element is given by tree graphs with one spectator nucleon, with LO vertices, $\mathrm{O}(\mathrm{P})$. This contribution is nonvanishing only for charged pion photoproduction. At this order one finds the Kroll-Ruderman term (Fig. 41a). $O\left(P^{2}\right)$ : The first corrections to the leading terms are the same as above but with $O\left(P^{2}\right)$ vertices. For example, there is a $1 / M$ correction at this order (Fig. 41 b). $O\left(P^{3}\right)$ : There are three

\footnotetext{
${ }^{m}$ In this calculation, the dominant isospin breaking effect, namely the charged to neutral pion mass difference has been taken into account. A fully consistent calculation including all effects from virtual photons and the quark mass differences is not yet available. The result for $E_{0+}^{\pi^{0} n}$ is based on the assumption that the counterterms entering at $O\left(P^{4}\right)$ are the same for the proton and the neutron apart from trivial isospin factors.
} 
classes of corrections at this order: (i) One-loop graphs with one spectator nucleon (Fig. 41c). (ii) Tree graphs with one spectator nucleon and with either one $O\left(P^{3}\right)$ vertex (Fig. 41 d) or two $O\left(P^{2}\right)$ vertices. In the case of neutral pion photoproduction, the counterterm graphs with one $O\left(P^{3}\right)$ vertex vanish, while graphs with two $O\left(P^{2}\right)$ vertices give rise to $1 / M^{2}$ corrections proportional to the nucleon magnetic moments. (iii) Tree graphs with an interaction between nucleons with only $O(P)$ vertices. These graphs are of two types: twonucleon graphs like the Feynman graph of Fig. 41e and time-ordered graphs such as those in Fig. 41f. $O\left(P^{4}\right)$ : There are many new contributions at this order, which can be constructed following the same procedure. Most of these are calculable corrections to the lower orders, but among the single-scattering diagrams for neutral pion photoproduction an undetermined counterterm appears.

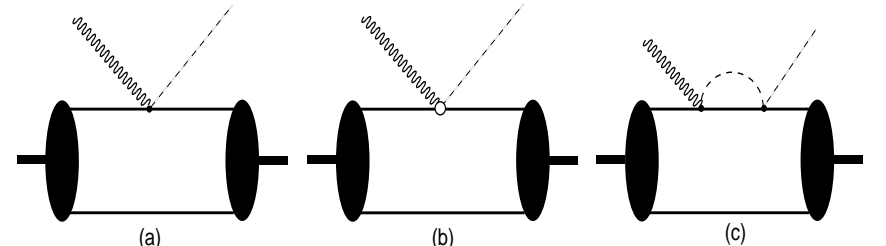

(a)

(b)

(c)

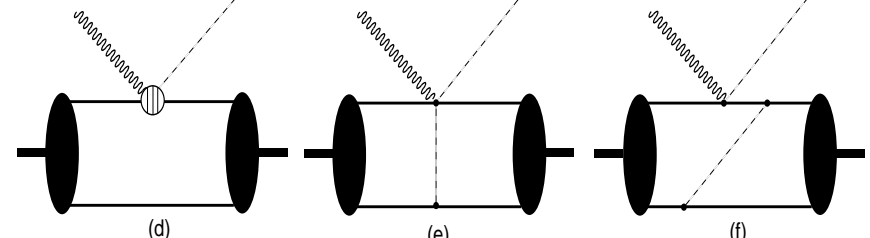

(e)

$(\mathrm{f})$

Figure 41: Characteristic graphs which contribute at each of the first three orders in pion photoproduction.

In the case of neutral pion photoproduction at threshold a number of the graphs in Fig. 41 will not contribute: those where the photon line is attached to a pion, and those that go like $S \cdot q$, where $q$ is the outgoing pion momentum and $S$ is the covariant nucleon spin. In particular, all the leading-order graphs (Fig. 41a) vanish, which immediately suggests that the cross-section will be smaller than that for charged pion production, and more sensitive to twonucleon contributions. Moreover, the time-ordered graphs (Fig. 41f) also vanish. It should be noted that contributions from the delta are minimized at threshold, and so integrating out the delta should not damage convergence of the theory too much. As noted above, to $O\left(P^{4}\right)$ there is one undetermined parameter, which is fitted to $\gamma p \rightarrow \pi^{0} p$ above threshold. 
We limit ourselves here to threshold neutral-pion photoproduction on the deuteron 155. As explained in the introduction, this is of special interest because it can provide information on the elementary neutron amplitude.

Consider the reaction $\gamma(k)+d\left(p_{1}\right) \rightarrow \pi^{0}(q)+d\left(p_{2}\right)$ in the threshold region, $\vec{q} \simeq 0$, where the pion is in an $S$ wave with respect to the center-of-mass $(\mathrm{cm})$ frame. For real photons $(\vec{\epsilon} \cdot \vec{k}=0$, with $\vec{\epsilon}$ the photon polarization vector), the differential cross-section at threshold takes the form

$$
\left.\frac{|\vec{k}|}{|\vec{q}|} \frac{d \sigma}{d \Omega}\right|_{|\vec{q}|=0}=\frac{8}{3} E_{d}^{2} .
$$

We now present the results of the chiral expansion of the dipole amplitude $E_{d}$ to $O\left(P^{4}\right)$. The single-scattering contribution is given by all diagrams where the photon and the pion are absorbed and emitted, respectively, from one nucleon with the second nucleon acting as a spectator (the impulse approximation). Some of these diagrams are depicted in Fig. 11. To $O\left(P^{4}\right)$ one finds

$$
\begin{aligned}
E_{d}^{s s} & =\frac{1+m_{\pi} / M}{1+m_{\pi} / m_{d}}\left\{\frac{1}{2}\left(E_{0+}^{\pi^{0} p}+E_{0+}^{\pi^{0} n}\right) \int d^{3} p \phi^{*}(\vec{p}) \vec{\epsilon} \cdot \vec{J} \phi(\vec{p}-\vec{k} / 2)\right. \\
& \left.-\frac{k}{M} \hat{k} \cdot \int d^{3} p \hat{p} \frac{1}{2}\left(P_{1}^{\pi^{0} p}+P_{1}^{\pi^{0} n}\right) \phi^{*}(\vec{p}) \vec{\epsilon} \cdot \vec{J} \phi(\vec{p}-\vec{k} / 2)\right\}
\end{aligned}
$$

evaluated at the threshold value

$$
|\vec{k}|=k_{\mathrm{thr}}=m_{\pi^{0}}\left(1-\frac{m_{\pi^{0}}^{2}}{2 m_{d}}\right)=130.1 \mathrm{MeV} .
$$

Here $m_{d}$ is the deuteron mass, $\vec{J}=\left(\vec{\sigma}_{1}+\vec{\sigma}_{2}\right) / 2$, and $\phi$ is the deuteron wavefunction.

Two remarks concerning Eq. (162) are in order:

(1) The elementary $S$-wave pion production amplitudes to $O\left(P^{4}\right) 176$ are given in Table 4 . The proton amplitude that results from a one-parameter fit above threshold compares well with experiment. The neutron amplitude is predicted to be considerably larger than the one obtained from the old "lowenergy theorem" that omits the important one-loop $O\left(P^{3}\right)$ single-scattering diagrams (Fig. 41c).

(2) It is important to differentiate between the $\pi^{0} d$ and the $\pi^{0} N(N=p, n)$ $\mathrm{cm}$ systems. At threshold in the former, the pion is produced at rest; it has, however, a small three-momentum in the latter 177, 178. Consequently, a singlenucleon $P$-wave contribution appears which is proportional to the elementary amplitudes $P_{1}^{\pi^{0} p}$ and $P_{1}^{\pi^{0} n}$ that obey the $P$-wave low-energy theorems 176 


$$
P_{1}^{\pi^{0} p}=0.480|\vec{q}| \mathrm{GeV}^{-2}, \quad P_{1}^{\pi^{0} n}=0.344|\vec{q}| \mathrm{GeV}^{-2}
$$

with

$$
\vec{q}=m_{\pi}\left[\left(1-\frac{m_{\pi}}{M}\right) \frac{\vec{p}}{M}-\frac{m_{\pi}}{2 M}\left(1-\frac{5 m_{\pi}}{4 M}\right) \hat{k}\right],
$$

where $\vec{p}$ is the nucleon momentum in the $\pi^{0} d \mathrm{~cm}$ system. The $P$-wave amplitude for neutral pion production off protons holds to within $3 \%$. Here we neglect the energy dependence of the elementary $\pi^{0} p$ and $\pi^{0} n S$ - and $P$-wave amplitudes since the pion energy changes only by $0.4 \%$ for typical average nucleon momenta in the deuteron.

The two-nucleon contributions at $O\left(P^{3}\right)$ that survive at threshold in the Coulomb gauge are shown in Fig. 42. All vertices come from the lowest order Lagrangian.

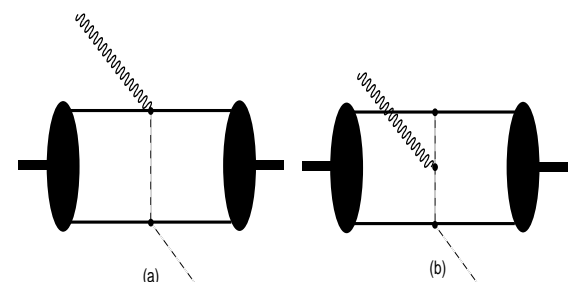

Figure 42: Two-nucleon graphs which contribute to neutral pion photoproduction at threshold to $O\left(P^{3}\right)$ (in the Coulomb gauge). All vertices come from $\mathcal{L}^{(0)}$.

At $O\left(P^{4}\right)$, we have to consider tree graphs with exactly one $O\left(P^{4}\right)$ vertex. The relevant terms in this Lagrangian fall into two classes, the first with fixed coefficients due to Lorentz invariance, the second with four low-energy constants $c_{1}, \ldots, c_{4}$ that are related to pion-nucleon scattering and two other constants that are related to the isoscalar and isovector nucleon anomalous magnetic moments. The pertinent two-nucleon diagrams are shown in Fig. 43, the blob characterizing an insertion from $\mathcal{L}_{\pi N}^{(2)}$. In addition, there are relativistic corrections to the graphs in Fig. 42 .

One can show that all terms proportional to the low-energy constants $c_{i}$ vanish at threshold leaving insertions of terms $\sim 1 / 2 M, \sim g_{A} / 2 M$ and $\sim \kappa_{0,1}$. Counting powers of momenta, one sees that the contributions from some graphs at this order are divergent. This is due to the fact that in the chiral expansion one has truncated the pion-nucleon form factor and thus does not suppress the 


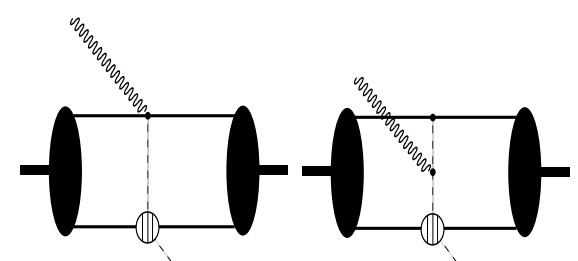

Figure 43: Characteristic two-nucleon graphs contributing at $O\left(P^{4}\right)$ to neutral pion photoproduction. The hatched circles denote an insertion from $\mathcal{L}_{\pi N}^{(2)}$.

high-momentum components. This phenomenon also occurs in the calculation of the $N N$ potential in $\chi P T 229.130$.131. As was done there, we introduce an additional Gaussian cut-off factor of the form $F\left(\vec{q}^{2}\right)=\exp \left\{-\vec{q}^{2} / \Lambda_{\pi}^{2}\right\}$, where the cut-off $\Lambda_{\pi}$ varies between the rho mass and $\Lambda_{\chi}$. Notice that the divergences appearing in other graphs all cancel and thus no further regularization is needed.

Note that to $O\left(P^{4}\right)$ there are no four-nucleon operators contributing to the deuteron electric dipole amplitude. The only possible graphs of this type that contribute at threshold to this order come from an $N N N N \pi$ vertex, to which a photon is attached either at the outgoing pion leg or at the vertex itself. Both vanish for neutral pions as they result from minimal substitution in a pion derivative. We therefore conclude that for threshold neutral pion photoproduction to $O\left(P^{4}\right)$ there is no contribution from any four-nucleon operator and thus no new, undetermingd parameters appear.

Using the Argonne V18 3 , the Reid 179, the Nijmegen 180 and the Paris 144.145 potentials, we find

$$
E_{d}^{s s}=(0.36 \pm 0.05) \times 10^{-3} / m_{\pi^{+}} \quad .
$$

The $P$-wave contribution amounts to a $3 \%$ correction to the $S$ wave. The sensitivity of the single-scattering contribution $E_{d}^{s s}$ to the elementary neutron amplitude is given by

$$
E_{d}^{s s}=\left[0.36-0.38 \cdot\left(2.13-E_{0+}^{\pi^{0} n}\right)\right] \times 10^{-3} / m_{\pi^{+}} .
$$

Consequently, for $E_{0+}^{\pi^{0} n}=0$, we have $E_{d}^{s s}=-0.45$ which is of opposite sign to the value in Eq. (166) based on the $\chi P T$ prediction for $E_{0+}^{\pi^{0} n}$ (Table 4 ). If 
one were to use the empirical value for the proton amplitude (Table \$), the single-scattering contribution would be somewhat reduced.

We find for the two-nucleon contribution at $O\left(P^{3}\right)$

$$
E_{d}^{t b, 3}=(-1.90,-1.88,-1.85,-1.88) \times 10^{-3} / m_{\pi^{+}},
$$

for the Argonne V18, Reid, super soft core 181 and Bonn 148 potentials, in order. Further, the result changes by only a few percent if the chiral potential of Ref. 131 is used 155 . From the graphs of $O\left(P^{4}\right)$ we find

$$
E_{d}^{t b, 4}=(-0.25,-0.23,-0.27) \times 10^{-3} / m_{\pi^{+}},
$$

for the Argonne V18, Reid and super soft core potentials, respectively, setting $\Lambda_{\pi}=1 \mathrm{GeV}$. These amount to corrections of the order of $15 \%$ to the $O\left(P^{3}\right)$ twonucleon terms. If one varies the cut-off $\Lambda_{\pi}$ from 0.65 to $1.5 \mathrm{GeV}$, the $O\left(P^{4}\right)$ twonucleon contribution using the Argonne V18 potential varies between -0.24 and -0.29 (in canonical units), which is a modest cut-off dependence.

In summary, taking the Argonne V18 potential for definiteness, the chiral expansion of the electric dipole amplitude $E_{d}$ is shown in Table 5 . Allowing $E_{0+}^{\pi^{0} p}$ to vary between -1.0 and -1.5 and for a fixed value of $E_{0+}^{\pi^{0} n}=2.13$, $E_{d}^{s s}$ varies between 0.2 and 0.4 (all numbers in canonical units). The results for the two-nucleon contributions are stable at $O\left(P^{3}\right)$, whereas we assign a conservative uncertainty of \pm 0.1 to the corrections at next order due to the cut-off dependence. We thus obtain the $O\left(P^{4}\right)$ prediction 155

$$
E_{d}=(-1.8 \pm 0.2) \times 10^{-3} / m_{\pi^{+}} .
$$

Table 5: Values for $E_{d}$ in units of $10^{-3} / m_{\pi^{+}}$from one-nucleon contributions $(1 N)$ up to $O\left(P^{4}\right)$, two-nucleon kernel $(2 N)$ at $O\left(P^{3}\right)$ and at $O\left(P^{3}\right)$, and their sum $(1 N+2 N)$.

\begin{tabular}{|cccc|}
\hline $1 N$ & \multicolumn{2}{c}{$2 N$} & $1 N+2 N$ \\
$P+P^{2}+P^{3}+P^{4}$ & $P^{3}$ & $P^{4}$ & $P+P^{2}+P^{3}+P^{4}$ \\
\hline 0.36 & -1.90 & -0.25 & -1.79 \\
\hline
\end{tabular}

To see the sensitivity to the elementary neutron amplitude, we set the latter to zero and find $E_{d}=-2.6 \times 10^{-3} / m_{\pi^{+}}$(for the Argonne V18 potential) which results in a cross-section twice as large as the one from the $\chi P T$ prediction (170). This is consistent with previous model results 177.178.

This is a unique situation: the EFT makes a prediction that differs significantly from conventional models. Such a test was recently carried out at 
Saskatoon 182. The experimental results for the pion photoproduction crosssection near threshold are shown in Fig. 44, together with the $\chi P T$ prediction at threshold. Inelastic contributions have been estimated in Ref. 182 and are smaller than $10 \%$ throughout the range of energies shown. At threshold, Ref. 182 extracts

$$
E_{d}=(-1.45 \pm 0.09) \times 10^{-3} / m_{\pi^{+}} .
$$

While agreement with $\chi P T$ to order $O\left(P^{4}\right)$ is not better than a reasonable estimate of higher-order terms, it is clearly superior to models. This is compelling evidence of the importance of chiral loops and of the consistency of nuclear EFT. A further test will come from an electroproduction experiment currently under analysis in Mainz.

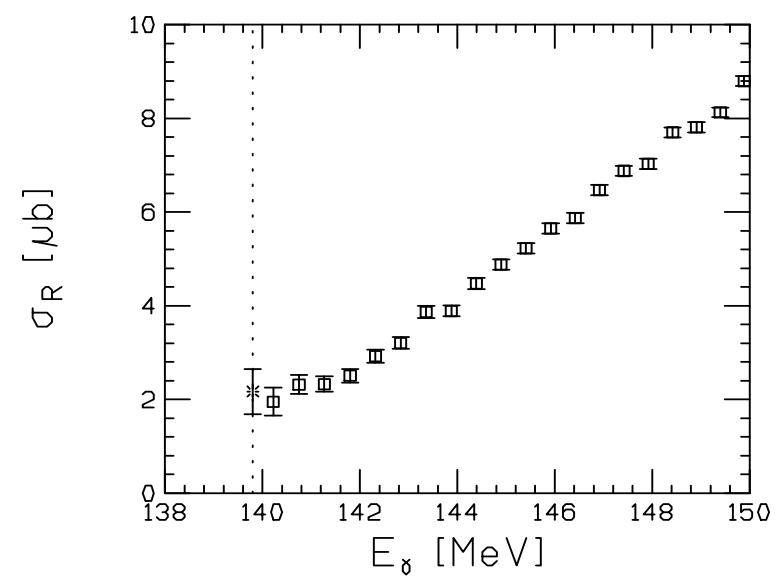

Figure 44: Reduced cross-section $\sigma_{R}=(k / q) \sigma$ in $\mu \mathrm{b}$ for neutral pion photoproduction as function of the photonergy in $\mathrm{MeV}$. Threshold is marked by a dpted line. Squares are data points from Ref. 182 and the star is the $\chi P T$ prediction of Ref. 155 . Figure courtesy of Ulf Meißner.

$8.6 \quad \gamma^{*} d \rightarrow \pi^{0} d$

In the case of an off-shell photon the threshold cross-section takes the form

$$
\sigma\left(k^{2}\right)=\left|E_{d}\right|^{2}+\epsilon_{L}\left|L_{d}\right|^{2},
$$

with $\epsilon_{L}=-\left(k^{2} / k_{0}^{2}\right) \epsilon$ the longitudinal polarization for a photon with polarization $\epsilon$. Now in addition to the transverse multipole $E_{d}$, there is a longitudinal multipole $L_{d}$ as well. Both of these multipoles are functions of the photon virtuality, $k^{2}$. 
These multipoles have been computed to $O\left(P^{3}\right)$ in the chiral expansion. To this order there are graphs with one spectator nucleon and a contact operator, together with the two graphs of Fig. 42 with an off-shell photon. The proton electric dipole amplitude at the photon point is not well described to this order. However, the $k^{2}$-dependence of the elementary amplitude for the proton is dominated by the third order contribution (see 183 for a discussion). The $\mathcal{O}\left(P^{3}\right)$ results for $E_{d}$ and $L_{d}$ are shown in Fig. 45 . In both cases, the two-body

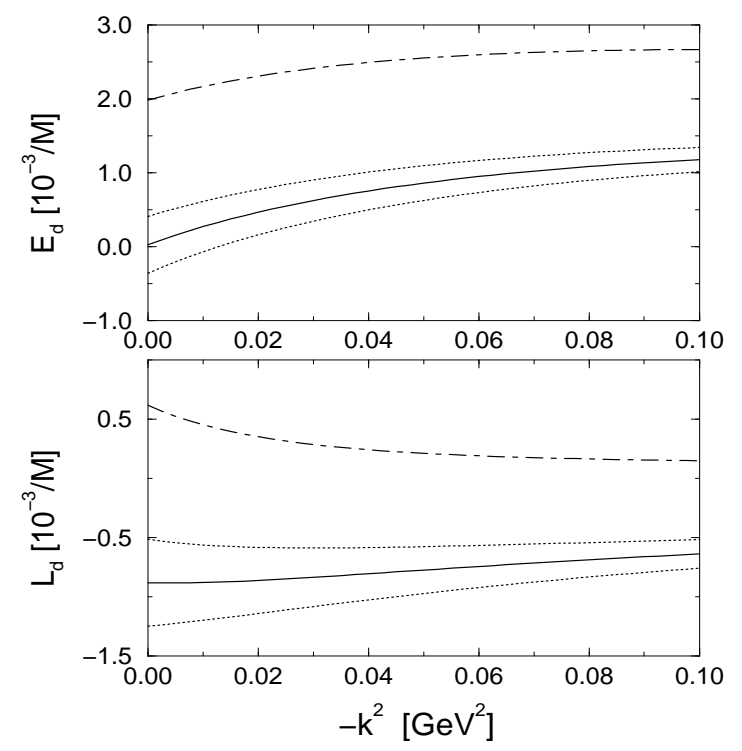

Figure 45: S-wave multipoles of the deuteron. In the upper and lower panel, the electric dipole and the longitudinal multipoles are shown, respectively, by the solid lines. The dotdashed line is the single scattering contribution. The dotted lines are obtained by changing the elementary neutron amplitude by $\pm 1 \times 10^{-3} / M_{\pi^{+}}$.

contribution is large and dominates the amplitudes. Note that $E_{d}$ varies more significantly with increasing $\left|k^{2}\right|$ than $L_{d}$. As illustrated by the dotted lines in Fig. 45, which have been obtained by a constant shift of the $\pi^{0} n$ amplitudes by $\pm 1 \times 10^{-3} / M_{\pi^{+}}$, there is sensitivity to the elementary scattering off the neutron. To get a more realistic estimate for the S-wave cross-section, one can adjust the values of $X_{0+}^{\pi^{0} p}$ and $X_{0+}^{\pi^{0} n}(X=\{E, L\})$ at the photon point to the values obtained from the $O\left(P^{4}\right)$ calculation. More precisely, the electric dipole amplitudes are taken from refl76, the $L_{0+}^{\pi^{0} p}$ from the best fit obtained in ref 183 and the $L_{0+}^{\pi^{0} n}$ using the resonance saturation estimate with $g_{3}=-125.6$ 
and $X^{\prime}=-0.23$, as detailed in 183 . The so calculated $\mathrm{S}-$ wave cross-section is collected in Table 6 for a photon polarization $\epsilon=0.67$. A range is given, which is obtained by adding a constant shift of $\pm 1 \times 10^{-3} / M_{\pi^{+}}$to the elementary $\pi^{0} n$ longitudinal multipole (but keeping the electric dipole amplitude at the same value as before). This serves to illustrate the sensitivity of the $\mathrm{S}$-wave cross-section to the so far unmeasured $L_{0+}^{\pi^{0} n}$ multipole For comparison, note that the $\mathrm{S}$-wave cross-section measured on the proton 184185 for $-0.10 \leq k^{2} \leq$ $-0.04 \mathrm{GeV}^{2}$ lies between 0.15 and $0.45 \mu \mathrm{b}$. For $k^{2} \simeq-0.1 \mathrm{GeV}^{2}, \sigma \simeq a_{0} / 2$, with $a_{0}$ the $\mathrm{S}$-wave cross-section for neutral pion production off the proton. However, the curvature of $\sigma\left(k^{2}\right)$ is rather different from the proton case. This is partly due to the interference of the proton and neutron amplitudes and partly a kinematical effect, since for a given polarization $\epsilon$ and virtuality $k^{2}$, $\epsilon_{L}$ is larger for the proton than for the deuteron. An $O\left(P^{4}\right)$ computation of this process is currently underway.

\begin{tabular}{|c||c|c|c|c|c|}
$-k^{2}\left[\mathrm{GeV}^{2}\right]$ & 0.02 & 0.04 & 0.06 & 0.08 & 0.10 \\
\hline$\sigma[\mu \mathrm{b}]$ & 0.11 & 0.15 & 0.17 & 0.19 & 0.20 \\
\hline$\sigma$ range $[\mu \mathrm{b}]$ & $0.09-0.13$ & $0.12-0.18$ & $0.14-0.21$ & $0.16-0.23$ & $0.17-0.24$
\end{tabular}

Table 6: S-wave cross-section for the scaled single scattering amplitudes as explained in the text. The range is obtained by adding a constant shift of $\pm 1 \times 10^{-3} / M_{\pi^{+}}$to the elementary $\pi^{0} n$ longitudinal multipole.

$8.7 \quad \pi d \rightarrow \pi d$

To $O\left(P^{3}\right)$ in $\chi P T$ the $\pi$-deuteron scattering length can be written as 9

$$
a_{\pi d}=\frac{(1+\mu)}{(1+\mu / 2)}\left(a_{\pi n}+a_{\pi p}\right)+a^{(2 a)}+a^{(2 b, 2 c)},
$$

where $\mu \equiv m_{\pi} / M$ is the ratio of the pion and the nucleon mass and the $\pi$-N scattering lengths have the decomposition $a_{\pi n}+a_{\pi p}=2 a^{+}=a^{(1 a)}+a^{(1 b)}$ where $a^{+}$is the isoscalar S-wave scattering length and the superscripts refer to the figures. The various diagrammatic contributions to $a_{\pi d}$ are illustrated in Fig. 46 and Fig. 47. The leading contribution, Fig. 46(a), has a vertex from $\mathcal{L}_{\pi N}^{(2)}\left(P^{2}\right)$ and is therefore $O\left(P^{2}\right)$. Fig. 46(b) has two nucleon propagators $\left(P^{-2}\right)$, one pion propagator $\left(P^{-2}\right)$, three vertices from $\mathcal{L}_{\pi N}^{(1)}\left(P^{3}\right)$ and a twobody factor $\left(P^{3}\right)$ and so gives an $O\left(P^{3}\right)$ contribution. One can further verify that the graphs of Fig. 47 are $O\left(P^{3}\right)$. Together, Figs. 46 and 47 are all that contribute at $O\left(P^{3}\right)$ at threshold. 


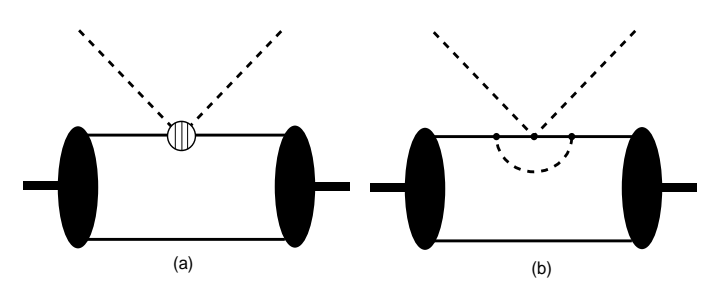

Figure 46: Contributions to the $\pi d$ scattering length at $\mathcal{O}\left(P^{2}\right)$ (a) and $\mathcal{O}\left(P^{3}\right)(\mathrm{b})$. The dots are vertices from $\mathcal{L}_{\pi N}^{(1)}$ and the sliced blob is from $\mathcal{L}_{\pi N}^{(2)}$.

The contributions to $a_{\pi d}$ from the graphs of Fig. 46 are:

$$
\begin{gathered}
a^{(2 a)}=-\frac{m_{\pi}^{2}}{32 \pi^{4} f_{\pi}^{4}(1+\mu / 2)}\left\langle\frac{1}{\vec{q}^{2}}\right\rangle_{\mathrm{wf}} \\
a^{(2 b, 2 c)}=\frac{g_{A}^{2} m_{\pi}^{2}}{128 \pi^{4} f_{\pi}^{4}(1+\mu / 2)}\left\langle\frac{\vec{q} \cdot \vec{\sigma}_{1} \vec{q} \cdot \vec{\sigma}_{2}}{\left(\vec{q}^{2}+m_{\pi}^{2}\right)^{2}}\right\rangle_{w f}
\end{gathered}
$$

where $\langle\vartheta\rangle_{\text {wf }}$ indicates that $\vartheta$ is sandwiched between deuteron wavefunctions. These matrix elements have been evaluated using a cornucopia of wavefunctions; results are displayed in Table 月. Clearly $a^{(2 a)}$ dominates. This is the result of the shorter range nature of $a^{(2 b, 2 c)}$. It is important to stress that the dominant contribution from these graphs is quite independent of the wavefunction used. This implies that $\chi P T$, which relies on the dominance of the pion-exchange, is useful in this context.

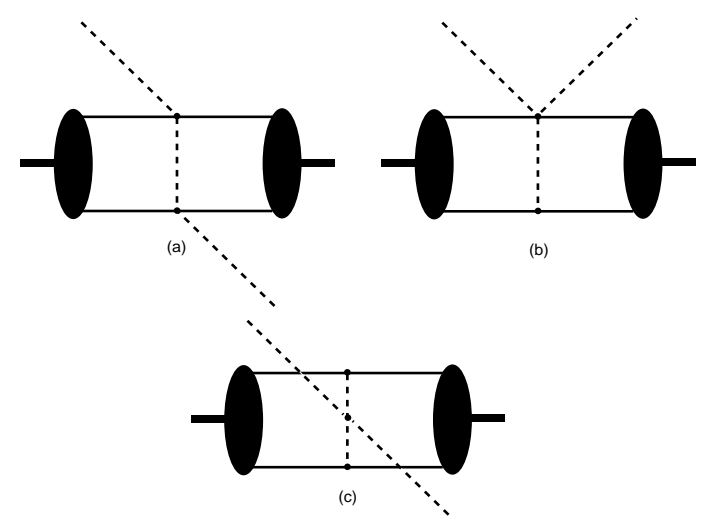

Figure 47: Contributions to the $\pi$-deuteron scattering length at $\mathcal{O}\left(P^{3}\right)$. 
Table 7: Contributions of Fig. 47 for various deuteron wavefunctions in units of $m_{\pi}^{-1}$. We use $f_{\pi}=92.4 \mathrm{MeV}, g_{A}=1.32$ and $m_{\pi^{+}}=139.6 \mathrm{MeV}$.

\begin{tabular}{|l|r|r|}
\hline$w f$ & $a^{(2 a)}$ & $a^{(2 b, 2 c)}$ \\
\hline Bonn148 & -0.02021 & -0.0005754 \\
ANL-V1: & -0.01960 & -0.0007919 \\
Reid_f6 & -0.01941 & -0.0008499 \\
SSC 181 & -0.01920 & -0.0006987 \\
\hline
\end{tabular}

To $O\left(P^{3}\right)$ in $\chi P T 186$ :

$$
4 \pi(1+\mu) a^{+}=\frac{m_{\pi}^{2}}{f_{\pi}^{2}}\left(-4 c_{1}+2 c_{2}-\frac{g_{A}^{2}}{4 M}+2 c_{3}\right)+\frac{3 g_{A}^{2} m_{\pi}^{3}}{64 \pi f_{\pi}^{4}}
$$

where the $c_{i}$ are low-energy constants from $\mathcal{L}_{\pi N}^{(2)}$. The sole undetermined parameter entering the $O\left(P^{3}\right)$ computation of $a_{\pi d}$ is therefore a combination of $c_{1}, c_{2}$ and $c_{3}: \Delta \equiv-4 c_{1}+2\left(c_{2}+c_{3}\right)$.

There is recentexperimental information about both the $\pi \mathrm{N}$ and $\pi d$ scattering lengths 187, 188, 189. Since $a^{+}$involves constants that are not fixed by chiral symmetry we can use experimental information about $\pi d$ scattering to predict $a^{+}$; the recent Neuchatel-PSI-ETHZ (NPE) pionic deuterium measurement 187 gives

$$
a_{\pi d}=-0.0259 \pm 0.0011 m_{\pi}^{-1} .
$$

For the contributions of Fig. 47 we take the average of the $a^{(2 a)}$ and $a^{(2 b, 2 c)}$ values in Table 8: $a^{(2 a+2 b+2 c)}=-0.0203 m_{\pi}^{-1}$. We then find from Eq. (173):

$$
a^{+}=-(2.6 \pm 0.5) \cdot 10^{-3} m_{\pi}^{-1} .
$$

Note that although $a^{(2 b, 2 c)}$ is small, there is a strong cancellation between $a^{(2 a)}$ and $a_{\pi d}$ which leads to a sensitivity to $a^{(2 b, 2 c)}$. Our value of $a^{+}$is not consistent with the Karlsruhe-Helsinki value $190, a^{+}=-(8.3 \pm 3.8) \cdot 10^{-3} m_{\pi}^{-1}$, or the new NPE value deduced from the strong interaction shifts in pionic hydrogen and deuterium, which is small and positive 188,189, $a^{+}=(0 \ldots 5) \cdot 10^{-3} m_{\pi}^{-1}$. The result Eq. (178) agrees, however, with the value obtained in the SM95 partial-wave analysis $191, a^{+}=-3.0 \cdot 10^{-3} m_{\pi}^{-1}$.

Given the ambiguous experimental situation regarding $a^{+}$, it seems most profitable to turn our formula around and use the $\pi d$ scattering data to constrain $\Delta$. We can write

$$
\Delta=\frac{2 \pi f_{\pi}^{2}}{m_{\pi}^{2}}(1+\mu / 2)\left\{a_{\pi d}-\left(a^{(2 a)}+a^{(2 b, 2 c)}\right)\right\}+\frac{g_{A}^{2}}{4 M}\left(1-\frac{3 M m_{\pi}}{16 \pi f_{\pi}^{2}}\right) .
$$


Using Eqs. (174), (175) and (177) we find $\Delta=-(0.08 \pm 0.02) \mathrm{GeV}^{-1}$, where we have taken into account the error in the determination of $a_{\pi d}$.

In Table 8 we give values of the relevant $c_{i}$ obtained from a realistic fit to low-energy pion-nucleon scattering data and subthreshold parameters 192. Central values lead to $\sigma(0)=47.6 \mathrm{MeV}$ and $a^{+}=-4.7 \cdot 10^{-3} m_{\pi}^{-1}$. These values of the $c_{i}$ give the conservative determination: $\Delta=-(0.18 \pm 0.75) \mathrm{GeV}^{-1}$. Also

Table 8: Values of the LECs $c_{i}$ in $\mathrm{GeV}^{-1}$ for $i=1, \ldots, 3$. Also given are the central values (cv) and the ranges for the $c_{i}$ from resonance exchange. The ${ }^{*}$ denotes an input quantity.

\begin{tabular}{|l|r|r|r|}
\hline$i$ & \multicolumn{1}{|c|}{$c_{i}$} & \multicolumn{1}{|c|}{$c_{i}^{\text {Res }} \mathrm{cV}$} & $c_{i}^{\text {Res }}$ ranges \\
\hline 1 & $-0.93 \pm 0.10$ & $-0.9^{*}$ & - \\
2 & $3.34 \pm 0.20$ & 3.9 & $2 \ldots 4$ \\
3 & $-5.29 \pm 0.25$ & -5.3 & $-4.5 \ldots-5.3$ \\
\hline$\Delta$ & $-0.18 \pm 0.75$ & 0.8 & $-3.0 \ldots+2.6$ \\
\hline
\end{tabular}

shown in Table 8 are values of the $c_{i}$ deduced from resonance saturation. It is worth mentioning that an independent fit to pion-nucleon scattering including also low-energy constants related to dimension three operators finds results consistent with the fit values of Table 8193.

\section{$8.8 \gamma d \rightarrow \gamma d$}

Nucleon Compton scattering has been studied in $\chi P T$ in Ref. 194, 195, where the following results for the polarizabilities were obtained at LO

$$
\begin{gathered}
\alpha_{p}=\alpha_{n}=\frac{5 e^{2} g_{A}^{2}}{384 \pi^{2} f_{\pi}^{2} m_{\pi}}=12.2 \times 10^{-4} \mathrm{fm}^{3} ; \\
\beta_{p}=\beta_{n}=\frac{e^{2} g_{A}^{2}}{768 \pi^{2} f_{\pi}^{2} m_{\pi}}=1.2 \times 10^{-4} \mathrm{fm}^{3} .
\end{gathered}
$$

Recent experimental values for the proton polarizabilities are 199

$$
\begin{aligned}
& \alpha_{p}+\beta_{p}=13.23 \pm 0.86_{-0.49}^{+0.20} \times 10^{-4} \mathrm{fm}^{3}, \\
& \alpha_{p}-\beta_{p}=10.11 \pm 1.74_{-0.86}^{+1.22} \times 10^{-4} \mathrm{fm}^{3},
\end{aligned}
$$

where the first error is a combined statistical and systematic error, and the second set of errors comes from the theoretical model employed. These values are in good agreement with the $\chi P T$ predictions.

\footnotetext{
${ }^{n}$ These are the result of a model-dependent fit to data from Compton scattering on the
} proton at several angles and at energies ranging from 33 to $309 \mathrm{MeV}$. 
On the other hand, the neutron polarizabilities are difficult to obtain experimentally due to the absence of suitable neutron targets and so the corresponding $\chi P T$ prediction is not well tested. One way to extract neutron polarizabilities is to consider Compton scattering on nuclear targets. Consider coherent photon scattering on the deuteron. The cross-section in the forward direction naively goes as:

$$
\left.\frac{d \sigma}{d \Omega}\right|_{\theta=0} \sim\left(f_{T h}-\left(\alpha_{p}+\alpha_{n}\right) \omega^{2}\right)^{2} .
$$

The sum $\alpha_{p}+\alpha_{n}$ may then be accessible via its interference with the dominant Thomson term for the proton, $f_{T h}$. This means that with experimental knowledge of the proton polarizabilities it may be possible to extract those for the neutron. Coherent Compton scattering on a deuteron target has been measured at $E_{\gamma}=49$ and $69 \mathrm{MeV}$ by the Illinois group 87 and, using tagged photons, in the energy range $E_{\gamma}=84.2-104.5 \mathrm{MeV}$ at Saskatoon 197. Data for $E_{\gamma}$ of about $60 \mathrm{MeV}$ is currently being analyzed at Lund.

Clearly the amplitude for Compton scattering on the deuteron involves mechanisms other than Compton scattering on the individual constituent nucleons. Hence, extraction of nucleon polarizabilities requires a theoretical calculation of Compton scattering on the deuteron that is under control in the sense that it accounts for all mechanisms to a given order in $\chi P T$. There exist a few calculations of this reaction in the framework of conventional potential models 198,299,200. These calculations yield similar results if similar input is supplied, but typically mechanisms for nucleon polarizabilities and two-nucleon contributions are not treated consistently. We will see that $\chi P T$ provides a framework where this drawback is eliminated.

The Compton amplitude we wish to evaluate is (in the $\gamma d$ center-of-mass frame):

$$
\begin{aligned}
& T_{M^{\prime} \lambda^{\prime} M \lambda}^{\gamma d}\left(\vec{k}^{\prime}, \vec{k}\right)=\int \frac{d^{3} p}{(2 \pi)^{3}} \psi_{M^{\prime}}\left(\vec{p}+\frac{\vec{k}-\vec{k}^{\prime}}{2}\right) T_{\gamma N_{\lambda^{\prime} \lambda}}^{\gamma d c . m \cdot}\left(\vec{k}^{\prime}, \vec{k}\right) \psi_{M}(\vec{p}) \\
& +\int \frac{d^{3} p d^{3} p^{\prime}}{(2 \pi)^{6}} \psi_{M^{\prime}}\left(\vec{p}^{\prime}\right) T_{\gamma N N_{\lambda^{\prime} \lambda}}^{2 N}\left(\vec{k}^{\prime}, \vec{k}\right) \psi_{M}(\vec{p})
\end{aligned}
$$

where $M\left(M^{\prime}\right)$ is the initial (final) deuteron spin state, and $\lambda\left(\lambda^{\prime}\right)$ is the initial (final) photon polarization state, and $\vec{k}\left(\vec{k}^{\prime}\right)$ the initial (final) photon threemomentum, which are constrained to $|\vec{k}|=\left|\vec{k}^{\prime}\right|=\omega$. The amplitude $T_{\gamma N}^{\gamma d}$ c.m. represents the graphs of Fig. 48 and Fig. $49 \mathrm{~b}$ where the photon interacts with 

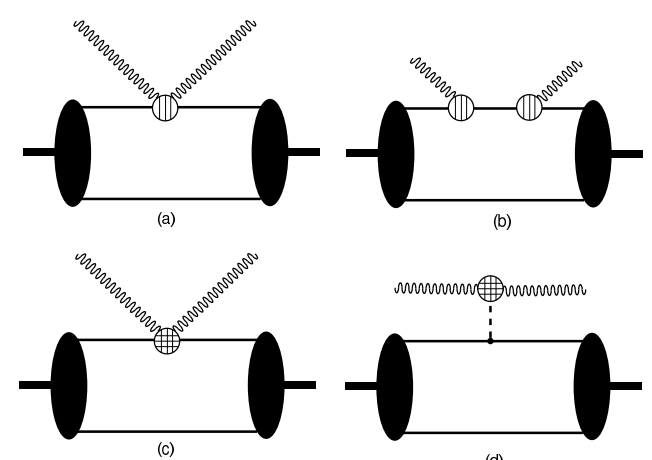

Figure 48: Graphs which contribute to Compton scattering on the deuteron at $\mathcal{O}\left(P^{2}\right)$ (a) and $\mathcal{O}\left(P^{3}\right)(\mathrm{b}-\mathrm{d})$. The sliced and diced blobs are from $\mathcal{L}_{\pi N}^{(3)}(\mathrm{c})$ and $\mathcal{L}_{\pi \gamma}^{(4)}(\mathrm{d})$. Crossed graphs are not shown.

only one nucleon. Of course this amplitude must be evaluated in the $\gamma d$ centerof-mass frame. The amplitude $T_{\gamma N N}^{2 N}$ represents the graphs of Fig. $49 \mathrm{~b}$ where there is an exchanged pion between the two nucleons.

The LO contribution to Compton scattering on the deuteron is shown in Fig. 48(a). This graph involves a vertex from $\mathcal{L}_{\pi \gamma}^{(2)}$ and so is $O\left(P^{2}\right)$. This contribution is simply the Thomson term for scattering on the proton. At next order, $O\left(P^{3}\right)$, there are several more graphs with a spectator nucleon Fig. $48(\mathrm{~b}, \mathrm{c}, \mathrm{d})$ and as well as graphs involving an exchanged pion with leading order vertices (Fig. 49a) and one loop graphy with a spectator nucleon (Fig. 49b). The full amplitudes are given in Ref.158.

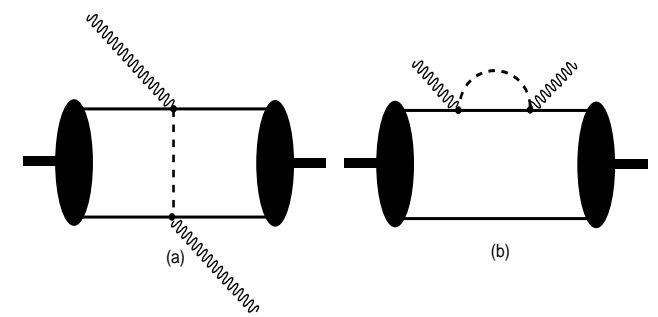

Figure 49: Graphs which contribute to Compton scattering on the deuteron at $\mathcal{O}\left(P^{3}\right)$. Crossed graphs are not shown.

For the wave function $\psi$ we use the energy-independent Bonn OBEPQ wave function parameterization which is found in Ref. 148 as well as the chiral wavefunction computed to $\mathrm{N}^{2} \mathrm{LO}$ in Ref. 138 . The photon-deuteron $T$-matrix 
in Eq. (183) is then calculated and the laboratory differential cross-section evaluated directly from it:

$$
\frac{d \sigma}{d \Omega_{L}}=\frac{1}{16 \pi^{2}}\left(\frac{E_{\gamma}^{\prime}}{E_{\gamma}}\right)^{2} \frac{1}{6} \sum_{M^{\prime} \lambda^{\prime} M \lambda}\left|T_{M^{\prime} \lambda^{\prime} M \lambda}^{\gamma d}\right|^{2},
$$

where $E_{\gamma}$ is the initial photon energy in the laboratory frame and $E_{\gamma}^{\prime}$ is the final photon energy in the laboratory frame. Fig. 50 shows the results at $E_{\gamma}=49,69$, and $95 \mathrm{MeV}$. For comparison we have included the calculation at $O\left(P^{2}\right)$, where the second contribution in Eq. (183) is zero, and the $\gamma N$ $T$-matrix in the single-scattering contribution is given by the Thomson term on a single nucleon. It is remarkable that to $O\left(P^{3}\right)$ no unknown counterterms appear. All contributions to the kernel are fixed in terms of known pion and nucleon parameters such as $m_{\pi}, g_{A}, M$, and $f_{\pi}$. Thus, to this order $\chi P T$ makes predictions for Compton scattering.

The curves show that the correction from the $O\left(P^{3}\right)$ terms gets larger as $\omega$ is increased, as was to be expected. Indeed, while at lower energies corrections are relatively small, in the $95 \mathrm{MeV}$ results the correction to the differential cross-section from the $O\left(P^{3}\right)$ terms is of order $50 \%$, although the contribution of these terms to the amplitude is of roughly the size one would expect from the power-counting: about $25 \%$. Nevertheless, it is clear, even from these results, that this calculation must be performed to $O\left(P^{4}\right)$ before conclusions can be drawn about polarizabilities from data at photon energies of order $m_{\pi}$. This is in accord with similar convergence properties for the analogous calculation for threshold pion photoproduction on the deuteron 155 .
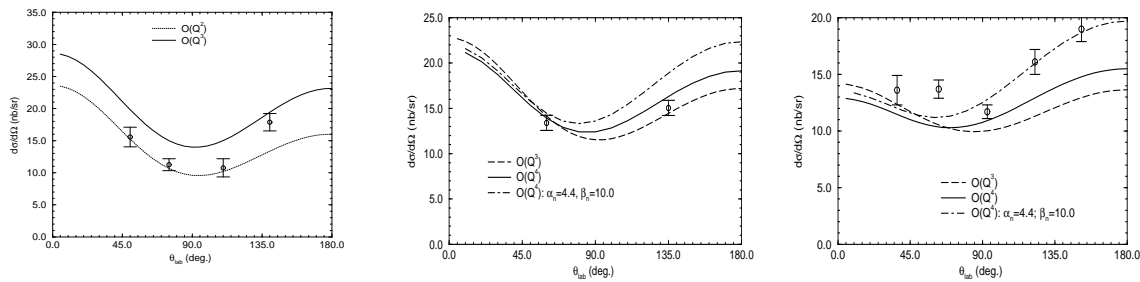

Figure 50: Results of the $O\left(P^{2}\right)$ (dotted line) and $O\left(P^{3}\right)$ (solid line) calculations for $E_{\gamma}=$ $49 \mathrm{MeV}, 69 \mathrm{MeV}$ and $95 \mathrm{MeV}$ respectively from left to right.

We have also shown the six Illinois data points at 49 and $69 \mathrm{MeV} 87$ and the Saskatoon data at $95 \mathrm{MeV} 201$. Statistical and systematic errors have been added in quadrature. It is quite remarkable how well the $O\left(P^{2}\right)$ calculation 
reproduces the $49 \mathrm{MeV}$ data. However, it is clear that the agreement at forward angles is somewhat fortuitous, as there are significant $O\left(P^{3}\right)$ corrections. Meanwhile, the agreement of the $O\left(P^{3}\right)$ calculation with the $69 \mathrm{MeV}$ data is very good, although only limited conclusions can be drawn, given that there are only two data points, each with large error bars.

These results are qualitatively not very different from other existing calculations. At 49 and $69 \mathrm{MeV}$ the $O\left(P^{3}\right)$ results are very close to those in Ref 198 and a few nb/sr higher, especially at back angles, than those of Refs. 199 200. At $95 \mathrm{MeV}$ the $O\left(P^{3}\right)$ result is close to that of Ref. 299 , higher by several $\mathrm{nb} / \mathrm{sr}$ at back angles than Ref. 200 , and several nb/sr lower than the calculation with no polarizabilities of Ref. 198 \%. However the EFT calculation is the only one to incorporate the full single-nucleon amplitude instead of its polarizability approximation. The tendency to higher relative cross-sections in the backward directions is at least in part due to this feature.

Although nominally the domain of validity of the Weinberg formulation extends well beyond the threshold for pion production, the power-counting fails at low energies well before the Thompson limit is reached. Consider the $O\left(P^{4}\right)$ contribution shown in Fig. 51. We can use this graph to illustrate the transition to the very-low energy regime $\sim m_{\pi}^{2} / M$. It is easy to see that this graph becomes comparable to the order $P^{3}$ graph of Fig. 49 b when $|\vec{p}|^{2} \sim \omega M$. Here $\vec{p}$ is a typical nucleon momentum inside the deuteron and $\omega$ is the photon energy. Since our power-counting is predicated on the assumption that all momenta are of order $m_{\pi}$, we find that power-counting is valid in the region $m_{\pi}^{2} / M \ll P \ll \Lambda_{\chi}$. Therefore, in the region $\omega \sim B$ the Weinberg powercounting is not valid, since the external probe momentum flowing through the nucleon lines is of order $P^{2} / M$, rather than order $P$. It is in this region that the Compton low-energy theorems are derived. Therefore our power-counting will not recover those low-energy theorems. Of course the upper bound on the validity of the effective theory should increase if the $\Delta$-resonance is included as a fundamental degree of freedome2.

There are $O\left(P^{4}\right)$ counterterms which shift the polarizabilities. We use this freedom to fit the SAL data using the incomplete $O\left(P^{4}\right)$ calculation. A reasonable fit at backward angles can be achieved with $\alpha_{n}=4.4$ and $\beta_{n}=10$, which are in startling disagreement with the $O\left(P^{3}\right) \chi P T$ expectations. That a fit can be achieved with these values of the polarizabilities is not surprising; continually increasing $\beta_{p}+\beta_{n}$ at approximately constant $\alpha_{p}+\alpha_{n}$ decreases the cross-section at forward angles and increases it at back angles. In fact, it seems that if $\beta_{p}+\beta_{n}$ is sufficiently large then the character of the cross-section

\footnotetext{
${ }^{o}$ At this energy Ref. 198 only presents results with $\alpha_{p}+\alpha_{n}=\beta_{p}+\beta_{n}=0$, whiej in turn are considerably less forward peaked than the corresponding calculation of Ref. 199.
} 


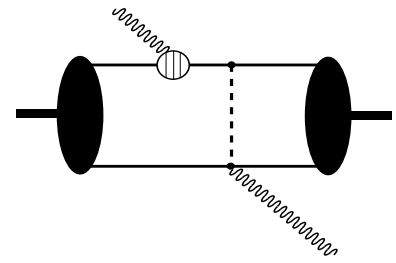

Figure 51: Interaction which contributes to Compton scattering on the deuteron at $\mathcal{O}\left(P^{4}\right)$. The sliced blob represents a $1 / M$ correction vertex from $\mathcal{L}_{\pi N}^{(2)}$.

at $95 \mathrm{MeV}$ can change completely from forward peaked to backward peaked. We have also plotted the cross-section at $69 \mathrm{MeV}$ with $\alpha_{n}=4.4$ and $\beta_{n}=10$. This curve is then excessively forward peaked and misses the Illinois data. This situation poses an interesting theoretical puzzle. Of course, a full $O\left(P^{4}\right)$ calculation in $\chi P T$ is necessary if the neutron polarizability is to be extracted from the Saskatoon data in a systematic way.

\section{$8.9 \quad n p \rightarrow d \gamma$}

The chiral filter hypothesis is an idea based on current algebra 203.204. The hypothesis is that when a long-wavelength photon or axial probe interacts with the nucleus the dominant meson-exchange current contribution comes from the interaction of the probe with the soft pions. The arguments given above for ed scattering suggest that, as first argued by Rho 205, this hypothesis can be organized into a systematic statement of the relative importance of various contributions to the $N N$ system current, using Weinberg power counting.

This can be clearly seen in the calculation of threshold $n p \rightarrow d \gamma$. In Ref.63.64 Park et al. computed the M1 isovector matrix element for this reaction up to $O\left(P^{5}\right)$ in Weinberg counting. At $O\left(P^{2}\right)$ the LO contribution to the M1 transition enters. This is just the isovector counterpart of the one-body operator given above in Eq. (154):

$$
\mu_{1 \mathrm{~B}}=\frac{e}{2 M}\left[\left(1+\kappa_{p}\right) \sigma_{p}+\kappa_{n} \sigma_{n}\right] .
$$

At $O\left(P^{3}\right)$ the two two-body currents depicted in Fig. 37 enter (although, of course, now a continuum $n p$ state is the final-state of the system). We will

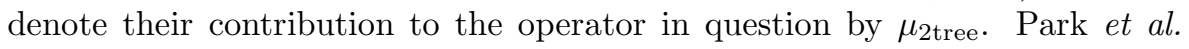
derive an analytic expression for this operator in co-ordinate space:

$$
\mu_{2 \text { tree }}=e \frac{g_{A} m_{\pi}^{2}}{8 f_{\pi}^{2}}\left(\vec{\tau}_{1} \times \vec{\tau}_{2}\right)\left\{\left(\sigma_{1} \times \sigma_{2}\right)\left[\frac{2}{3}\left(1+m_{\pi} r\right)-1\right]\right.
$$




$$
\left.-\left[\hat{r} \hat{r} \cdot\left(\sigma_{1} \times \sigma_{2}\right)-\frac{1}{3}\left(\sigma_{1} \times \sigma_{2}\right)\right]\left[1+m_{\pi} r\right]\right\} \frac{e^{-m_{\pi} r}}{m_{\pi} r} .
$$

This exchange current was derived many years ago by Chemtob and Rho 203 , but it now has a definite place in the expansion of the two-nucleon current in powers of the small parameter $P$.

The correction at next order, $O\left(P^{4}\right)$, vanishes, and so the next term in the series occurs at $O\left(P^{5}\right)$. At this order three types of term enter.

1. Loop corrections to the $\pi N N$ and $\gamma \pi N N$ vertices. Thus, the only corrections comes from the counterterms which renormalize the divergences that appear in the loop graphs contributing to the $\gamma \pi N N$ vertex. Park et al. chose to fix these counterterms using resonance saturation. These low-energy constants could also be obtained by fitting to data in the single-nucleon sector. The topological structure of these graphs is the same as the left-hand graph in Fig. 52, and these contributions are denoted by $\mu_{2 \text { vertex }}$.

2. There are also a number of one-loop, two-body exchange currents (not shown) denoted by $\mu_{2 l o o p}$.

3. There is the right-hand graph in Fig. 52. This is a contact interaction arising from the Lagrangian (73). Park et al. argue that this contribution is suppressed in their "hard-core cutoff scheme" (HCCS) 63664. However, if this process was reconsidered in the light of their more recent work, where a "modified hard-core cutoff scheme" (MHCCS) was employed, then the effect of this graph would be non-zero.

Without this last contribution the magnetic moment operator is just the sum of all of these contributions, $\mu=\mu_{1 \mathrm{~B}}+\mu_{2 \text { tree }}+\mu_{2 \text { vertex }}+\mu_{2 \text { loop }}$ each of which is calculable in terms of $g_{A}, f_{\pi}, \kappa_{N}$, or low-energy constants from the single-nucleon sector. This operator must now be sandwiched between deuteron and $n p$ scattering state wave functions. For this purpose Park et al. employed the AV18 wave functions. The contribution from the one-body operator $\mu_{1 B}$ gave a cross-section for threshold neutron capture, $\sigma_{1 \mathrm{~B}}=305.6 \mathrm{mb}$, very close to the result from ERT 60. This simply means that the threshold capture cross-section is dominated by the physics of the $N N$ wave function tail, which is the same in ERT and the AV18 potential.

The evaluation of the two-body magnetic-moment operators is a little more subtle. We write the contribution of any given operator $\mu$ to the M1 matrix 
element as:

$$
\delta_{X}=\frac{\left\langle d\left|\mu_{X}\right| n p\right\rangle}{\left\langle d\left|\mu_{1 \mathrm{~B}}\right| n p\right\rangle} .
$$

Park et al. cut-off the contribution from the operators $\mu_{X}$ at $r=r_{c} \sim 1 / \Lambda$, since the physics of this short-distance region will not be well-represented in $\chi \mathrm{PT}$. Multiplying the operators written above by $\theta\left(r-r_{c}\right)$ and employing the AV18 wave functions they can then evaluate the ratios $\delta$ as functions of $r_{c}$. The dependence on $r_{c}$ is rather weak for a range of cutoffs from $r_{c}=0$ to $r_{c}=0.7 \mathrm{fm}$. Summarizing the results at $r_{c}=0.6 \mathrm{fm} 64 \delta_{2 \text { tree }}=2.592 \%$, $\delta_{2 \text { vertex }}=1.477 \%$, and $\delta_{2 \text { loop }}=0.362 \%$. This leads to a theoretical prediction: $\sigma=334 \pm 3 \mathrm{mb}$, in excellent agreement with the experimental cross section $\sigma^{\text {th }}$.

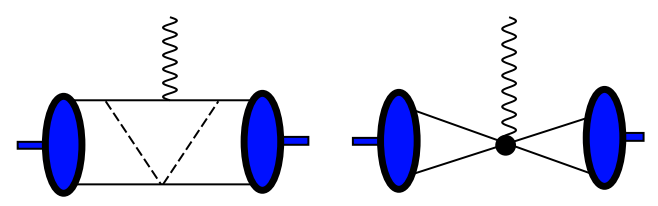

Figure 52: Two two-body currents which contribute to $E 2_{S}$ and $M 1_{S}$ at $O\left(P^{5}\right)$.

As in the case of $\operatorname{EFT}(\hbar)$ this approach can also be used to evaluate the suppressed amplitudes $M 1_{S}$ and $E 2_{S} 68$. The results obtained agree with those discussed previously, but differ somewhat in the error estimates.

\section{$8.10 p p \rightarrow d e^{+} \nu_{e}$}

Similar computations were performed in Ref. 159 for the solar proton burning process $p p \rightarrow d e^{+} \nu_{e}$. The dominant piece comes from the one-body axial current. Park et al. show that this contribution can be evaluated with low-energy experimental data, $\rho_{d}$, and $\eta_{s d}$, as long as one is careful to include Coulomb corrections in the $p p$ wave function. This gives a result: $\mathcal{M}_{1 B}^{\exp }=4.858 \pm 0.006 \mathrm{fm}$, where we have included the errors from the experimental data, added linearly. In contrast direct evaluation of the matrix element with the AV18 wave function yields: $\mathcal{M}_{1 B}^{\mathrm{AV} 18}=4.859 \mathrm{fm}$. Neither of these numbers include a "theorydependent" part of the matrix-element which comes from the interference between the Coulomb and strong nuclear $p p$ interactions. Using AV18 wave functions this additional piece of $\mathcal{M}_{1 B}$ is computed to be: $\mathcal{M}_{1 B}^{C+N}=0.011 \mathrm{fm}$. The contribution of vacuum polarization in the $p p$ final state is also small, of order $0.5 \%$ of $\mathcal{M}_{1 B}$. Effects of two-photon exchange are smaller still. In fact, 
all of these numbers are robust under the interchange of the AV18 wave function with an EFT-motivated wave function, provided that in the EFT wave function the short-distance interaction is tuned to reproduce the scattering length and effective range of the $p p$ system.

The strength of these short-distance pieces is unconstrained by chiral symmetry, although we expect it to be "natural". As in Ref.64, in Ref. 159 it was argued that this counterterm contribution to $p p \rightarrow d e^{+} \nu_{e}$ will be suppressed by the presence of the hard core in the $N N$ interaction. It would be interesting to see how the use of the MHCCS would modify the conclusions of Ref.159. However, as in $\operatorname{EFT}(\hbar)$, this would require some way to determine the counterterm contribution. This would need to be done either by measuring a low-energy neutrino cross-section in the $N N$ system, or by calculating the rate for the $\beta$-decay of tritium, including the counterterm, and then fixing the counterterm to reproduce the extremely well-known experimental number, as discussed previously.

In Ref. 159 only the axial currents of "pion range" were evaluated. These gave a contribution of between 2 and $5 \%$ to the overall matrix element, depending on what cutoff radius was employed. It would seem that this strong cutoff dependence might be due to the omission of the corresponding counterterm from the calculation. Regardless, the number resulting from this $\chi \mathrm{PT}$ calculation is still accurate at the level of a few per cent. Park et al. quote results for the astrophysical S-factor 70 .

$$
S_{p p}(E)=\sigma(E) E e^{2 \pi \eta}
$$

where $\sigma$ is the cross-section for $p p \rightarrow d e^{+} \nu_{e}$, and $\eta=M \alpha /(2 p)$. The value obtained in Ref. 159 is:

$$
S_{p p}(0)=\left(4.05 \times 10^{-25}\right)(1 \pm 0.0015 \pm 0.0048)^{2} \mathrm{MeV} \text { barn }
$$

where the first error is due to the uncertainty in the one-body matrix element and the second is Park et al.'s estimate of the error on the two-body matrix element.

In contrast the canonical value of Kamionkowski and Bahcall206, calculated using a variety of $N N$ potential models, is:

$$
S_{p p}(0)=\left(3.89 \times 10^{-25}\right)(1 \pm 0.011) \mathrm{MeV} \text { barn }
$$

The more sophisticated potential-model calculation of Ref. 71 gives a much smaller error bar

$$
S_{p p}(0)=\left(3.88 \times 10^{-25}\right)(1 \pm 0.003) \mathrm{MeV} \text { barn }
$$


where the error comes both from a combination of small effects neglected in the calculation of the $N N$ wave functions and from the observed sensitivity to the choice of modern $N N$ potential.

\subsection{Pion Production}

The pion production reactions: $p+p \rightarrow p+p+\pi^{0}, N+N \rightarrow d+\pi$, and $N+N \rightarrow p+n+\pi$ would seem to be a natural candidate for calculation in $\chi \mathrm{PT}$. However, the situation here is complicated by the appearance of a new scale. Since the energy of the incoming nucleons in any of these processes is of order $m_{\pi}$ the typical nucleon momentum in the initial state is $19 \sqrt{M m_{\pi}}$. This renders the $\chi \mathrm{PT}$ expansion for these processes poorly convergent, since the expansion is now one in $\sqrt{m_{\pi} / M} \sim 0.4$. This makes it difficult to reproduce the experimentaldata, which is in fact of very high quality, in both the case of neutral pion 19 and charged pion 207 production. There have been many attempts to treat the S-wave prequction of pips to high-enough order that some level of convergence is seen 208,209.210.211,212 213, but, at this stage it seems that the series is just not well-behaved.

One solution to this is to resum certain mechanisms in the $\chi \mathrm{PT}$ expansion in some non-systematic way, as discussed in Ref. 214.215. Alternatively, one can turn to the production of $\mathrm{P}$-wave pions. There one might hope that the series is better behaved, since the $\mathrm{LO}$ contribution is large 216. The results from early studies of the production of $\mathrm{P}$-wave pions in $\chi \mathrm{PT}$ are encouraging, but the expansion parameter remains large. The advantage of employing $\chi \mathrm{PT}$ to study the production of $\mathrm{P}$-wave pions is that, if the series is well-behaved enough, the experimental data might be able to be employed to extract information on contact operators involving four nucleons and a single pion. It is exactly this type of operator which enters in the novel three-nucleon forces discussed in Ref.217. The study of $\mathrm{P}$-wave pion production therefore provides us with an opportunity to use $\chi \mathrm{PT}$ to systematically connect the three-nucleon system with pion-production in the two-nucleon system.

\subsection{Three-Body Forces}

In the Weinberg program the order in which the three-body forces start contributing depends on whether or not one is including the isobars explicitly. Let us first discuss the case where the $\Delta$ 's are present 218 . The three-body forces then first appear at order $\mathcal{O}\left(P^{2}\right)$ and are given by tree diagrams involving $\Delta$ 's as intermediate states and zero, one and two pion exchanges. At order $\mathcal{O}\left(P^{3}\right)$ three classes of contributions appear. The first involves two-pion exchanges and a one-nucleon-two-pion vertex. The three independent one-nucleon-two-pion 
vertices can be fixed by the study of pion-nucleon scattering. The second class involves one pion exchange and a two-nucleon-one-pion vertex. The two independent coefficients of the two-nucleon-one-pion vertices can be determined by analyzing pion-deuteron scattering and/or pion production in nucleon-nucleon scattering. One particular linear combination of these two coefficients was determined by considering $p$-wave pion production in $N+N \rightarrow N+N+\pi 219$. It is also known that if those coefficients have natural size they can appreciably affect the vector-analyzing power $A_{y}$ in proton-deuteron scattering that is not very well described by phenomenological two-body forces only 220. Finally the last category includes contact three-body pieces that can only be determined by fitting to three-body observables. In the version of the theory where the isobars are integrated out the three-body forces have no contribution at order $P^{2}$ and start at order $P^{3}$. The contributions have the same form as the case with $\Delta$ 's, but with different coefficients.

Only recently the first calculations of three-body observables in the Weinberg scheme were performed A calculation at the order $P^{2}$ without explicit $\Delta$ 's shows two main features 221. The binding energy of the triton is found to be close to the experimental value but shows a cutoff dependence that seems to be too large for a NLO calculation. The values obtained were $B_{3}=8.284$ for $\Lambda=540 \mathrm{MeV}$ and $B_{3}=7.546$ for $\Lambda=600$, but with a $10 \%$ variation in the binding energy for a $10 \%$ variation in the cutoff. It is presently unclear whether cutoff dependence is somewhat accidental or indicates that three-body forces that could cancel this dependence appear at lower order than predicted by Weinberg's power-counting, as shown to be the case in the pionless theory. The second feature is that spin-observables are well described by the NLO calculation, including the vector-analyzing power $A_{y}$.

\subsection{Formal Problems with Weinberg Power Counting}

As we have already discussed, at LO in Weinbergs power-counting there are contributions to $V_{0}^{(2)}$ from both the local four-nucleon operators, $C_{S, T}$ and from the exchange of a single potential pion, giving a momentum space potential of

$$
V_{0}^{(2)}\left(\mathbf{p}, \mathbf{p}^{\prime}\right)=C-\left(\frac{g_{A}^{2}}{2 f_{\pi}^{2}}\right) \frac{\left(\mathbf{q} \cdot \sigma_{1} \mathbf{q} \cdot \sigma_{2}\right)\left(\tau_{1} \cdot \tau_{2}\right)}{\left(\mathbf{q}^{2}+m_{\pi}^{2}\right)},
$$

where $C$ denotes the combination of $C_{S, T}$ appropriate for a given spin-isospin channel. The LO amplitude results from solving the Schrödinger equation with this potential. This can be justified in the EFT only if $M \sim P^{-1}$, otherwise diagrams at different orders in Weinbergs expansion are numerically of the same size. At two-loops in the ladder sum there is a logarithmic divergence in the 


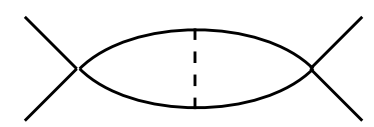

(a)

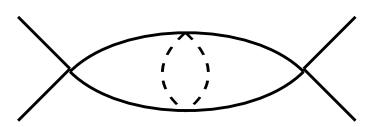

(b)

Figure 53: Graphs with logarithmic divergences at LO in Weinberg's power counting. The solid lines are nucleons and the dashed lines are pions.

graph shown in Fig. 53 a that must be regulated. In dimensional regularization the divergent part of this graph is

$$
-\frac{1}{\epsilon} \frac{g_{A}^{2} m_{\pi}^{2} M^{2}}{128 \pi^{2} f_{\pi}^{2}} C^{2}
$$

which requires a counterterm with a single insertion of the light quark mass matrix, for instance

$-\frac{1}{2} C_{S}^{(m)} \operatorname{Tr}\left[m_{q}\left(\Sigma+\Sigma^{\dagger}\right)\right]\left(N^{\dagger} N\right)^{2}-\frac{1}{2} C_{T}^{(m)} \operatorname{Tr}\left[m_{q}\left(\Sigma+\Sigma^{\dagger}\right)\right]\left(N^{\dagger} \sigma N\right)^{2}+\ldots$.

However, the coefficients of these operators must scale like $M^{2}$, and since $m_{\pi}^{2} M^{2} \sim P^{0}$ these formally higher order operators in Weinberg's powercounting are required at LO to absorb divergences in the time-ordered products of the LO potential, $V_{0}^{(2)}$. Ignoring the multi-pion vertices arising from these operators, they can be re-absorbed into the LO operators with coefficients $C_{S, T}$. We are then in the situation where there is no chiral expansion, multiple insertions of the light quark mass matrix are not suppressed compared to LO interactions.

The Schrödinger equation sums diagrams that are of different order in $P$. Paradoxically, it is consistent with Weinberg's power counting not to use the Schrödinger equation. In that case the near threshold bound states and the associated behavior of the scattering amplitudes are not recovered. It was shown in Ref. 18 that taking $M \sim P^{-1}$ corrects this problem. However, a more intricate scaling might in fact be in operation.

Resolving these issues is currently the most important line of investigation in multi-nucleon EFT, since only then will there be an acceptable formal basis for the EFT at scales $|\mathbf{k}| \gtrsim m_{\pi}$. 


\section{Gains over the Traditional Approach}

Traditionally, low-energy phenomena in light nuclei have been described using the Schrödinger equation with a model for the $N N$ potential. In practice, all sensible potentials include the one-pion exchange potential (OPEP) as their long-range tail. The inclusion of OPEP is required on theoretical grounds, as it uniquely describes the longest-range part of the strong interaction. This can also be seen experimentally, since OPEP is essential in the description of the higher partial waves in $N N$ scattering 146,17 . On the other hand, apart from their common one-pion exchange tails, different $N N$ potential models look rather dissimilar. Some include a set of single (or, in some cases, double) boson-exchanges to generate the short-distance piece, the interaction, as in the Nijmegen 180 or the original Bonn potentials 149,222 . Others have amore phenomenological,short-distance structure, e.g. the Reid potential the 179 , the Paris potential 144 145, or the Argonne potentials 5 .223. In all cases the shortdistance potential must be rich enough to describe the existing data. Once a particular form is chosen the Schrödinger equation is solved and the free parameters are fit to the $N N$ scattering data and certain deuteron boundstate properties. This is now an extremely sophisticated enterprise. Potentials exist which fit the $n p$ and $p p$ scattering data up to laboratory energies of 350 $\mathrm{MeV}$ with a $\chi^{2}$ per degree of freedom close to one 4 ⿶.

The treatment of short-distance physics employed in many of these potential models can be motivated from an EFT point of view Potentials ranging from the modern AV18 0 to the venerable Reid potential 179 are based on the idea that the details of the short-distance physics in the $N N$ problem are not important. It is important to introduce a structure for the short-distance operators that parameterizes our ignorance about that part of the interaction, but the detailed form of that parameterization is not crucial. This is perhaps not surprising: physically it is just not possible to resolve the details of short-distance physics using low-momentum observables. Therefore attempts to get the "right" short-distance $N N$ potential using low-momentum data are futile. Potential models have predictive power precisely because the details of the short-distance physics do not matter greatly.

It should therefore be clear that the goal of a $\chi \mathrm{PT} N N$ potential is not to learn about the short-distance structure of the $N N$ interaction. The chiral Lagrangian quite deliberately makes no dynamical assumptions about dynamics

${ }^{p}$ The CD-Bonn potential 224 6 nominally involves single-meson exchanges at short distances, but the mass of the scalar-isoscalar meson varies from partial wave to partial wave. Therefore in classifying OBE potentials as we have done here it is not entirely clear which class to place it in. 
at scales of order $\Lambda_{\chi}$, and so, by construction, the $\chi \mathrm{PT} N N$ potential contains only gross information about the physics which takes place at this scale. There is, however, the chance to learn something about the pion physics which is the provenance of $\chi \mathrm{PT}$.

Of course, "traditional" potential models are not just employed to fit $N N$ scattering data. They also do a very good job in describing a wide array of low-energy data obtained using electroweak probes. To calculate the response of the nucleus to these probes, single-nucleon currents and potential-model wave functions are often employed. In some cases such a construction does not lead to a conserved electromagnetic current, and so additional "modelindependent" fwo-body currents are added to restore current conservation (see,

for instance 165 ). Conversely, transverse currents are not constrained by current conservation and so are always "model-dependent". The usual practice is to model these currents via something akin to the resonance saturation hypothesis used to estimate counterterms in the single-nucleon sector of $\chi \mathrm{PT}$. The results for these transverse currents reflect dynamical assumptions about the important physical mechanisms, e.g. that pionic, $\rho$-meson, $\rho \pi \gamma, \omega \pi \gamma, \Delta \pi \gamma$, and $\Delta \rho \gamma$ meson-exchange currents dominate the transverse currents.

Employing $\chi \mathrm{PT}$ to calculate these current operators leads to two gains over these more standard approaches. Firstly, as in the case of the $N N$ potential, it simplifies the picture of the dynamics down to its essential features: the "long-distance" part of the current operator comes from the physics of pions and nucleons interacting with the probe, while the short-distance part must be parameterized in some way. Secondly, and perhaps of more practical importance, the calculation of current operators in $\chi \mathrm{PT}$ systematizes the treatment of the current operators in the $N N$ system. In particular, $\chi \mathrm{PT}$ constrains the number and form of current operators allowable at a given order and mandates the type of pion-loop and pion-exchange mechanisms which should be included in the calculation. This means that, as in all EFT calculations, errors can be estimated in a reliable way. Sych error estimation is possible in potential-model calculations (see, for instance 71 ), but it is significantly simpler in $\chi \mathrm{PT}$.

\section{The Nuclear Many-Body Problem as an EFT}

In order for the EFTs that we have discussed in the previous pages of this chapter to have any impact whatsoever on nuclear structure, a way of solving the many-body problem must be developed, that is consistent with the power counting. As we do not know exactly what the final EFT will look like in the momentum region relevant to nuclei, we now turn our attention to the problem of solving the nuclear many-body problem for the hamiltonian given 
in Eq. (11). The hope is that the EFT program will allow enable us to match onto Eq. (11) and provide the tools to systematically calculate corrections to it. Equally important is that the many-body problem has to be solved anyway at some point! Although this problem has consumed nuclear physicists for several decades, the tools are still not available to make reliable calculations in bound systems with more than $\sim 10$ nucleons. Furthermore the techniques employed in systems of 6-10 nucleons - Green's function Monte Carlo (GFMC) and variational methods - are sufficiently technical and challenging numerically that very few theorists have access to them. In still heavier systems the techniques of choice, such as the shell model, are phenomenological, providing no basis for systematic corrections or evaluating errors. For these reasons there is some interest in the possibility that the ideas of EFTs may point the way to new many-body techniques that are both simpler and more systematic and controlled. Although the first such steps in this direction have been taken only recently here we summarized some recent work (that of Haxton and collaborator 225.226) (for others investigations of multi-nucleon dynamics see e.g. Ref.227,228) which suggests that conventional nuclear models do have controlled effective theory counterparts in which modern techniques, such as the renormalization group, can be employed to simplify the classical nuclear physics problem of point nucleons interacting through a static potential. A related and very interesting development that we will not discuss involves similar progress in systematically treating low-density nuclear system,229,230. The use of dimensional regularization in this scenario leads to great simplifications in various calculations.

The canonical microscopic model for nuclear structure - the shell model (SM) - is motivated in many textbooks by Brueckner's treatment of nuclear matter. The language is at least superficially reminiscent of an EFT. The starting point is a collection of nonrelativistic nucleons interacting through a potential, e.g., of the Nijmegen or Argonne/Urbana type within an infinite Hilbert space

$$
H=\frac{1}{2} \sum_{i, j=1}^{A}\left(T_{i j}+V_{i j}\right),
$$

where $T_{i j}$ is the relative nonrelativistic kinetic energy operator and $V_{i j}$ the nucleon-nucleon potential. The related SM Hamiltonian acts in a restricted space and employs a softer "effective" potential,

$$
H_{S M}=\frac{1}{2} \sum_{i, j=1}^{A}\left(T_{i j}+V_{i j}^{e f f}\right) .
$$


Motivating $H_{S M}$ is the notion that the determination of $V^{e f f}$ might be simpler than solving the original A-body problem: the foundation of Brueckner theory is that high-momentum contributions to the wave function might be integrated out in a rapidly converging series in $\rho_{\text {nuclear }}$ or, equivalently, in the number of nucleons in high-momentum states interacting at one time outside the SM space. This assumption - sometimes termed the hole-line expansion - clearly has some connections with our discussion of the power-counting for three-body forces, and thus may find some justification through EFTs.

As will become apparent later, a typical SM space will contain explicitly $\sim 60 \%$ of the wave function that resides at long-wavelengths, thus guaranteeing at least a qualitative description of A-body correlations important to soft collective modes. Implicitly the high-momentum components are swept into a rather poorly defined "effective interaction," often determined empirically. The strength of the SM resides in the first of these two aspects: the technology developed for direct diagonalizations in large SM spaces is quite remarkable, including recent progress in Lanczos-based methods 231, in treatments of light nuclei involving many shells232, and in Monte Carlo sampling 233234. Its weakness is the numerous uncontrolled approximations that become apparent when one tries to view the shell model as a faithful effective theory (ET). However, we show below that the same numerical strides that have advanced shell model diagonalizations now allow us to remove these uncontrolled approximations. The resulting ET has many differences with the shell model and many similarities to the EFTs discussed previously.

Among the SM uncontrolled approximations are the following: 1) Even in LO, where only the pairwise interaction of high-momentum nucleons is included in $H^{e f f}$, the functional form of the resulting effective interaction is not as simple as assumed in the SM,

$$
\left\langle\left|H^{e f f}\right|\right\rangle_{S M} \equiv\left\langle\alpha \beta\left|H^{e f f}\right| \gamma \delta\right\rangle
$$

where the Greek symbols label single-particle shell-model states. As nucleons in high-momentum states can scatter pairwise repeatedly, this expression can at best be valid in some LO sense: beyond LO three-, four-, and higher-body body operators must be successively induced. Furthermore the energy denominators describing this scattering depend on the total many-body energ.235, not simply the single-particle states participating in the scattering (apart from special cases, like a LO calculation restricted to a single shell). Thus the SM simplification of $H^{e f f}$ is ad hoc.

2) SM wave functions are orthogonal and normed to unity. In ET the effective wave functions are naturally defined as the restrictions of the true wave 
functions $\left|\Psi_{i}\right\rangle$ to the model space

$$
\left|\Psi_{i}\right\rangle \underset{E T}{\longrightarrow}\left\langle S M \mid \Psi_{i}\right\rangle|S M\rangle \equiv\left|\Psi_{i}^{e f f}\right\rangle .
$$

Thus the norms are less than unity and orthogonality, which holds for the full wave functions, is lost for their restricted counterparts.

3) Shell model interactions frequently depend on fictitious parameters such as "starting energies," introduced to adjust the energy denominators in the highmomentum ladder diagrams. Answers depend on these parameters, as well as on others (e.g., the oscillator strength if the SM is defined in terms of harmonic oscillator Slater determinants, and the number of included oscillator quanta) that define the division between the SM and excluded high-momentum spaces. Clearly the predictions of a correct ET must be independent of all such physically irrelevant parameters.

4) Perhaps most serious, the important issue of effective operators is almost never addressed in a meaningful way. In many cases practitioners adopted a phenomenological $H^{e f f}$ which, while successful in producing spectra, provides no diagrammatic basis for calculating effective operators or wave function normalizations. Even in cases where $H^{\text {eff }}$ is derived from some underlying NN interaction, the practice is generally to then employ bare operators. In some well-studied cases, such as allowed $\beta$ decay in the $1 p$ and $2 s 1 d$ shells, it is then recognized that a phenomenological renormalization (e.g., $g_{A} \rightarrow 1$ ) of operators greatly improves agreement with experiment. But the origin of this renormalization and its evolution with momentum transfer $q$ are left unclear. The situation is very unsatisfactory and undercuts the shell model as a predictive tool.

\subsection{Self-consistent Bloch-Horowitz Solutions}

We consider the canonical nuclear structure problem of nonrelativistic point nucleons interacting through a realistic NN interaction, such as the Nijmegen potential discussed previously, or the similar Argonne V18 $\mathrm{C}$ or Reid93 179 potentials. The question is whether the uncontrolled approximations in the shell model can be removed, leaving a more complicated but still tractable effective theory. The approach involves three major steps:

- Formulating a treatment of effective interactions and operators that exploits the basic assumption in Brueckner theory - that interactions at high momenta can be integrated out in a cluster expansion (essentially an expansion in $\rho a^{3}$, where $\rho$ is the nuclear density and $a$ an interaction range) — but is otherwise 
exact. The convergence of the expansion could then be tested numerically and should depend on the operator under study and the momentum transfer. Alternatively (and preferably) one might be able to make connections to EFTs that would justify the expansion.

- To find numerical tricks for implementing this formulation, demonstrating their validity in cases (e.g, $\mathrm{A}=2,3,4)$ where the expansion can be carried to all orders, so that the answers should then agree with Faddeev and other exact methods.

- To imbed the formulation in a heavier nucleus, where the cluster expansion can be carried out only partially.

There is some reason for optimism that if the first two goals can be achieved, the third might yield very accurate results: the Argonne group cluster variational Monte Carlo effort on ${ }^{16} \mathrm{O}$ appeared to yield nearly exact results when clusters up to $\mathrm{A}=5$ were included. Below we describe progress toward the first two goals, contrasting an exact ET of the deuteron and ${ }^{3} \mathrm{He}$ with the shell model to illustrate the shortcomings of the later.

The approach is sketched in Fig. 54. The Hilbert space is divided into a long-wavelength "SM" space, defined by some energy scale $\Lambda_{S M}$, and a highmomentum space that extends to some scale $\Lambda_{\infty}$. Potential models effectively contain ultraviolet cutoffs embodied in their hard but finite short-range cores. Thus if $\Lambda_{\infty}$ is set to $\sim 3 \mathrm{GeV} / \hbar \omega \sim 140$ for the potentials mentioned above, ground state energies will be accurate to better than $1 \mathrm{keV}$. That is, for sufficiently large $\Lambda_{\infty}$

$$
H_{(i)}^{e f f}\left(\Lambda_{S M}, \Lambda_{\infty}\right) \underset{\Lambda_{\infty} \text { large }}{\longrightarrow} H_{(i)}^{e f f}\left(\Lambda_{S M}\right) .
$$

All correlations within the "SM" space are included, but the high-momentum correlations in the excluded space are limited to n-body, where $\mathrm{n}$ is the cluster size. Thus the lowest order effective interaction is

$$
H_{(n=2)}^{e f f} \equiv H_{(n=2,0)}^{e f f}
$$

It corresponds to embedding the A-body ladder diagram of Fig. 54 (b) between SM states: A-2 of the nucleons are spectators, with the remaining pair scattering via a two-body ladder. The notation $(n=2,0)$ states that the two-body cluster has no explicit dependence on the nuclear density, varying as $\rho^{0}$. The $\mathrm{n}=3$ A-body ladder of Fig. 54(c) is similarly

$$
H_{(n=3)}^{e f f} \equiv H_{(n=2,1)}^{e f f}+H_{(n=3,0)}^{e f f}
$$


where $H^{e f f}(n=2,1)$ is the two-body part of the three-body ladder

$$
\left\langle\alpha \beta\left|H_{(n=2,1)}^{e f f}\right| \alpha^{\prime} \beta^{\prime}\right\rangle=\sum_{\gamma \leq k_{F}}\left\langle\alpha \beta \gamma\left|H_{(n=3)}^{e f f}\right| \alpha^{\prime} \beta^{\prime} \gamma-\alpha^{\prime} \gamma \beta^{\prime}+\ldots\right\rangle,
$$

where $k_{F}$ denotes the Fermi level. This decomposition - which is done only to emphasize the content of the cluster expansion - illustrates that $H_{(n=3)}^{e f f}$ contains $H_{(n=2,0)}^{e f f}$ as well as a correction to the two-body interaction that depends linearly on the density and is obtained by identifying one ingoing SM single-particle leg of the three-body ladder with one outgoing leg, summed over all occupied states. It also contains a true three-body piece $H_{(n=3,0)}^{e f f}$, where three SM ingoing single-particle states connect to three distinct outgoing states after undergoing a series of scatterings outside the SM space. The point is simple pedagogy: treatments of successively larger clusters in the high-momentum space correct the lowest-order two-body $H_{(n=2,0)}^{\text {eff }}$ by adding terms proportional to the $\rho, \rho^{2}$, etc., in the spirit of Brueckner theory. It also adds true threebody terms, true four-body terms, etc. Thus an expansion through four-body clusters yields $H_{(n=2,2)}^{e f f}$, the two-body interaction corrected through order $\rho^{2}$, $H_{(n=3,1)}^{e f f}$, the three-body interaction through order $\rho$, and $H_{(n=4,0)}^{\text {eff }}$, a densityindependent true four-body interaction. We emphasize that the convergence of such an expansion is still a matter of conjecture, despite its physical plausibility and some justification from EFT power counting. The procedure has not yet been given a chance to succeed because the individual steps - certainly those beyond the two-body level - have not been carried out. We now discuss procedures that might make such an expansion feasible.

The calculation begins with a definition of the "SM" space. The goals of handling bound states and of generating an effective interaction that is translationally invariant leaves one sensible choice, many-body states constructed from harmonic oscillator Slater determinants. To exploit the relative/center-ofmass separability of harmonic oscillator Slater determinants, one must separate the SM and high-momentum spaces so that all configurations satisfying

$$
E \leq \Lambda_{S M} \hbar \omega
$$

are retained in the former. For example, a SM calculation of ${ }^{16} \mathrm{O}$ with $\Lambda_{S M}=$ $4+\Lambda_{0}$, where $\Lambda_{0}$ is the number of quanta in the ${ }^{16} \mathrm{O}$ closed shell, would include all $4 \hbar \omega$ configurations, e.g., $0 p 0 h, 2 p 2 h$, and $4 p 4 h$ excitations of nucleons from the $1 p$ shell into the $2 s 1 d$ shell, $1 p 1 h$ excitations of a $1 s$ shell nucleon into the $3 s 2 d 1 g$ shell, etc. One can define the projection operator onto the highmomentum space by

$$
Q_{S M}=Q\left(\Lambda_{S M}, b\right) .
$$




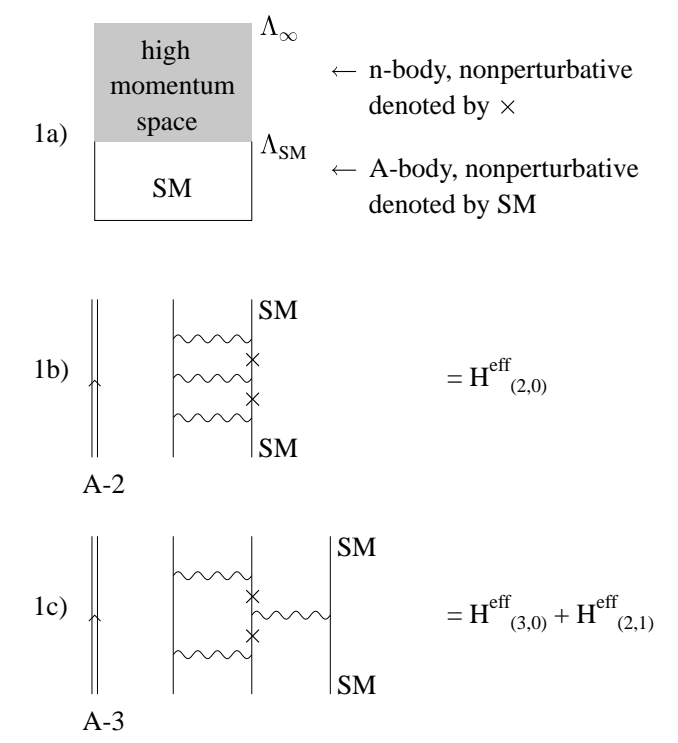

Figure 54: Cluster expansion of the effective interaction.

where $b$ is the oscillator parameter. Thus the included or "SM" space is defined by two parameters, $\Lambda_{S M}$ and $b$. The preservation of translational invariance is also important numerically, as it reduces the two-body ladder to an effective one-body problem, etc.

The resulting Bloch-Horowitz $(\mathrm{BH})$ equation 236 is then

$$
\begin{aligned}
H^{e f f} & =H+H \frac{1}{E-Q_{S M} H} Q_{S M} H \\
H^{e f f}\left|\Psi_{S M}\right\rangle & =E\left|\Psi_{S M}\right\rangle \quad\left|\Psi_{S M}\right\rangle=\left(1-Q_{S M}\right)|\Psi\rangle
\end{aligned}
$$

where $|\Psi\rangle$ is the exact wave function and $H|\Psi\rangle=E|\Psi\rangle$. The difficulty posed by this equation is the appearance of the unknown energy eigenvalue in the equation for $H^{e f f}$. Thus this system must be solved self-consistently. Note that there is no explicit reference to the harmonic oscillator in this equation: it enters only implicitly through $Q_{S M}$ in distinguishing the long-wavelength "SM" space from the remainder of the Hilbert space. We emphasize that a proper solution of the $\mathrm{BH}$ equation must yield results (energies and operator matrix elements) that are independent of $Q_{S M}$.

There is an extensive literature on this and similar equations, often involv- 
ing a division of $H$ into an unperturbed $H_{0}$ and a perturbation $H_{1}=H-H_{0}$ 237238. There are well-known pathologies with this division involving the effects of near-by intruder states on the perturbation expansion 239.240. Here we explore another approach that is nonperturbative and involves, in effect, a computer summation of diagrams. The method is based on the Lanczos algorithm and offers a remarkably simple solution to the issue of self-consistency.

In the Lanczos algorithm a basis for representing a Hamiltonian is formed recursively in such a way that the resulting Hamiltonian is tridiagonal. Given a Hermitian operator $H$ and and an initial normalized vector $\left|v_{1}\right\rangle$, the successive steps are

$$
\begin{array}{rrr}
H\left|v_{1}\right\rangle & =\alpha_{1}\left|v_{1}\right\rangle+\beta_{1}\left|v_{2}\right\rangle \\
H\left|v_{2}\right\rangle & =\beta_{1}\left|v_{1}\right\rangle+\alpha_{2}\left|v_{2}\right\rangle+\beta_{2}\left|v_{3}\right\rangle \\
H\left|v_{3}\right\rangle & =\quad \beta_{2}\left|v_{2}\right\rangle+\alpha_{3}\left|v_{3}\right\rangle+\beta_{3}\left|v_{4}\right\rangle \text { etc. }
\end{array}
$$

so that the $H$ takes the form

$$
H \rightarrow\left(\begin{array}{cccc}
\alpha_{1} & \beta_{1} & 0 & \cdots \\
\beta_{1} & \alpha_{2} & \beta_{2} & \cdots \\
0 & \beta_{2} & \alpha_{3} & \cdots \\
\vdots & \vdots & \vdots &
\end{array}\right)\left(\begin{array}{c}
\left|v_{1}\right\rangle \\
\left|v_{2}\right\rangle \\
\left|v_{3}\right\rangle \\
\vdots
\end{array}\right)
$$

The remarkable property of this algorithm has to do with truncating the process in Eq. (206) after $n$ steps, where $n$ can be much smaller than the dimension of the Hilbert space. The resulting truncated matrix in Eq. (207) then contains the information needed to reconstruct the exact $2 n-1$ lowest moments of $\mathrm{H}$ over the eigenspectrum. As extremum eigenvalues are crucial to higher moments, one common application of the Lanczos algorithm is in determining such eigenvalues and their associated eigenfunctions. Another is to begin with the vector $\left|v_{1}\right\rangle=\hat{O}|g . s$.$\rangle and then use the algorithm to calculate the moments$ of the response of the ground state $\mid g . s$. $\rangle$ to the operator $\hat{O}$. A small number of moments, e.g., $\sim 100$, often is sufficient to construct a response function with a numerical resolution comparable to that achieved experimentally.

A third application 241 is in constructing fully interacting Green's functions. One finds

$$
\frac{1}{E-H}\left|v_{1}\right\rangle=g_{1}(E)\left|v_{1}\right\rangle+g_{2}(E)\left|v_{2}\right\rangle+\cdots
$$

where the $g_{i}(E)$ are continued fractions that depend on $\alpha_{i}, \beta_{i}$ and where $\mathrm{E}$ 
appears only as a parameter. For example,

$$
g_{1}(E)=\frac{1}{E-\alpha_{1}-\frac{\beta_{1}^{2}}{E-\alpha_{2}-\frac{\beta_{2}^{2}}{E-\alpha_{3}-\beta_{3}^{2}} \cdots}}
$$

It follows that the $\mathrm{BH}$ equation can be solved self-consistently with only a single solution of the effective interactions problem, even in cases where multiple bound states are needed. The procedure is:

- For each relative-coordinate vector in the SM space $|\gamma\rangle$, form the excludedspace vector $\left|v_{1}\right\rangle \equiv Q_{S M} H|\gamma\rangle$ and the corresponding Lanczos matrix for the operator $Q_{S M} H$. Retaining the resulting coefficients $\alpha_{i}, \beta_{i}$ for later use, construct the Green's function for some initial guess for $E$ and then the dot product with $\left\langle\gamma^{\prime}\right| H$ to find $\left\langle\gamma^{\prime}\left|H^{e f f}(E)\right| \gamma\right\rangle$.

- Perform the "SM" calculation to find the desired eigenvalue $E^{\prime}$ which, in general, will be different from the guess $E$. Using the stored $\alpha_{i}, \beta_{i}$, recalculate the Green's function for $E^{\prime}$ and $H^{\text {eff }}\left(E^{\prime}\right)$ then redo the "SM" calculation. Repeat until convergence, i.e., until the input $E^{\prime}$ in the Green's function equals the desired output "SM" eigenvalue.

- Then proceed to the next desired bound state, e.g., the first excited state, and repeat the above step. Note that it is not necessary to repeat the $H^{e f f}$ calculation. The eigenvalue taken from the "SM" calculation is, of course, that of the first excited state. The procedure then generates distinct $H^{e f f}\left(E^{\prime}\right) \mathrm{s}$ for each desired state.

The attractiveness of this approach is that the effective interactions part of the procedure, which is relatively time consuming as it requires one to perform a large-basis Lanczos calculation for each relative-coordinate starting vector in the "SM" space, is performed only once. The diagonalization in the model space is generally much faster: modern workstations can handle even largedimension shell model calculations (sparse matrices of $\left.d \sim 10^{6}\right)$ quickly $(\sim 30$ minutes). In practice we found that self-consistency is achieved easily: six to eight cycles is typical. (More cycles are required for states with small binding energies.) Thus it is quite practical to derive the exact $H^{e f f}(E) \mathrm{s}$ for a series of bound states.

In our view the Lanczos approach to the effective interactions problem appears to be remarkably simpler than the standard procedures of the field, particular in view of the need for extensions to multi-nucleon ladders. The 
traditional approach divides the effective interactions problem into an energydependent piece (often called the Q-box) and an energy-dependent one (represented by folded diagrams). Frequently the energy dependence is removed by making a unitary transformation to a nonHermitian effective Hamiltonian. The Lanczos procedure is also much more in the spirit of EFT: the full Hamiltonian in the high-momentum space is never constructed. Rather, it is replaced by a much smaller Lanczos matrix which contains exactly the most relevant long-wavelength information of the full matrix, the $2 n-1$ lowest moments. Thus the procedure can be viewed as a numerical ET in which this information is recursively extracted.

Now we discuss the results of applying this procedure to the simplest nuclei, $\mathrm{d}$ and ${ }^{3} \mathrm{He}$, carrying the above process to completion (two-body and three-body ladders, respectively). The motivation is two-fold: demonstrate the numerical procedures we described above, and provide for the first time exact effective theory results that can be compared to those of the shell model.

The harmonic oscillator mode expansion must be sufficient to represent both the long-distance tails of bound states and the short-distance "hard core" scattering predicted by realistic NN potentials. (The Argonne V18 ${ }^{1} S_{0}$ potentials are shown in Fig. 55.) Inclusion of high-momentum states through $\Lambda_{\infty} \sim 50$ yields a deuteron binding energy accurate to $\sim 60 \mathrm{keV}$; extending this to $\sim 140$ produces a result accurate to one keV. Fig. 56 shows the rate of convergence as a function of $b$ and $\Lambda_{\infty}$. The convergence for the binding of ${ }^{3} \mathrm{He}$ (not shown) is slightly more rapid, a feature related to the deeper binding, as will become clear later.

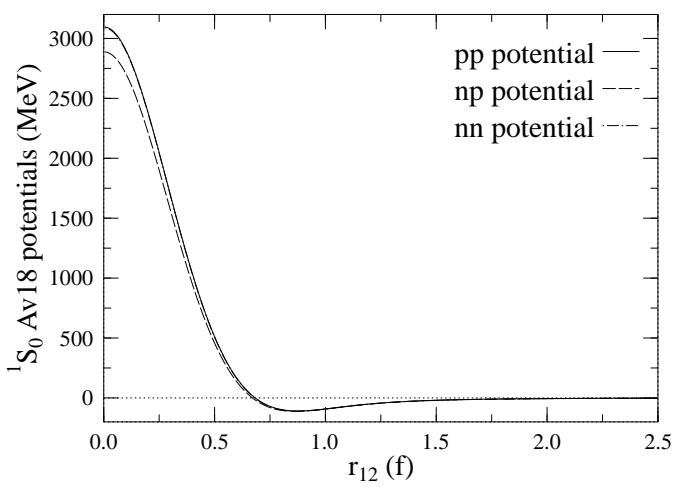

Figure 55: The Argonne V18 ${ }^{1} S_{0}$ potentials. 
The binding energies and operator matrix elements for simple systems like ${ }^{3} \mathrm{He}$ can, of course, be calculated by other methods, e.g., Faddeev techniques or GFMC. We thus want to stress that the point of the following discussion is to do analogous calculations in the context of an effective theory, so that we begin to see the shortcomings of conventional techniques like the shell model as well as possibilities for overcoming those shortcomings. A first test of the techniques outlined above is to solve the BH equation for some SM-like space to then see if the resulting self-consistent energy is, indeed, the correct value. For model spaces of $2,4,6$, and $8 \hbar \omega$ in the case of the deuteron we obtained a binding energy of $-2.224 \mathrm{MeV}$ (using $\sqrt{2} b=1.6 f$ and $\Lambda_{\infty}=140$ ). The exact result is $-2.2246 \mathrm{MeV}$. Our ${ }^{3} \mathrm{He}$ result for same SM spaces and $\Lambda_{\infty}=60$ is $-6.87 \mathrm{MeV}$, in agreement with the corresponding GFMC result of $-6.87 \pm 0.03$ $\mathrm{MeV}$.

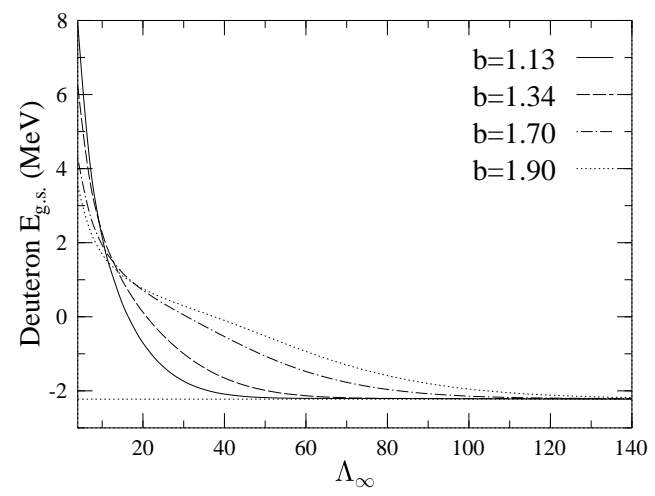

Figure 56: Deuteron ground state energy convergence as a function of $\Lambda_{\infty}$ for several choices of the oscillator parameter $b$. This $\mathrm{b}$ is defined as in the independent-particle SM: the corresponding $\mathrm{b}$ for relative motion is $\sqrt{2}$ times the values shown. It is clear that if an inappropriate size is chosen for the basis states (e.g., $b=1.9$ ), the rate of convergence can be greatly slowed.

More interesting is the evolution of the wave functions, shown in Tables 9 and 10. The tables were generated as above: for some large value of $\Lambda_{\infty}$ SM wave functions were generated for various choices of $\Lambda_{S M}$ by interating the SM calculation until the self-consistent energy is fully converged. The deuteron and ${ }^{3} \mathrm{He}$ calculations involve two- and three-body ladder sums in the excluded space, yielding sets of two- and three-body "SM" matrix elements of $H^{e f f}$ for the model spaces. The deuteron calculation is rather trivial; for $\Lambda_{\infty} \sim 50$ the ${ }^{3} \mathrm{He} \mathrm{BH}$ calculation involves a dense matrix of dimension $\sim 10^{4}$, 
Table 9: ET refor the deuteron ground state wave function calculated with the Argonne V18 potential 225.226. The columns on the right correspond to different choices of the ET model space, the analog of a SM space. The rows correspond to the resulting amplitudes for the designated, selected configurations $\left|n,{ }^{S} L_{J}\right\rangle$. The quantities within the parentheses are the square of the norm of the effective wave function, e.g., the probability that the deuteron resides in the corresponding "SM" space.

\begin{tabular}{|c|r|r|r|r|r|r|}
\hline \hline \multirow{2}{*}{ basis state } & \multicolumn{7}{|c|}{ amplitude } \\
\cline { 2 - 7 } & $0 \hbar \omega$ & $2 \hbar \omega$ & $4 \hbar \omega$ & $6 \hbar \omega$ & $8 \hbar \omega$ & exact \\
\cline { 2 - 7 } & $(65.9 \%)$ & $(79.5 \%)$ & $(86.1 \%)$ & $(91.3 \%)$ & $(93.0 \%)$ & $(100 \%)$ \\
\hline$\left|1,{ }^{3} S_{1}\right\rangle$ & 0.81155 & 0.81154 & 0.81155 & 0.81155 & 0.81152 & 0.81155 \\
\hline$\left|2,{ }^{3} S_{1}\right\rangle$ & 0.00000 & -0.31483 & -0.31483 & -0.31483 & -0.31482 & -0.31483 \\
\hline$\left|1,{ }^{3} D_{1}\right\rangle$ & 0.00000 & 0.19524 & 0.19524 & 0.19524 & 0.19523 & 0.19524 \\
\hline$\left|3,{ }^{3} S_{1}\right\rangle$ & 0.00000 & 0.00000 & 0.24945 & 0.24945 & 0.24944 & 0.24945 \\
\hline$\left|4,{ }^{3} S_{1}\right\rangle$ & 0.00000 & 0.00000 & 0.00000 & -0.20851 & -0.20850 & -0.20851 \\
\hline$\left|5,{ }^{3} S_{1}\right\rangle$ & 0.00000 & 0.00000 & 0.00000 & 0.00000 & 0.12596 & 0.12596 \\
\hline \hline
\end{tabular}

still rather modest by current SM standards. The matrix is dense because we work in relative Jacobi coordinates, rather than an $\mathrm{m}$-scheme, utilizing standard Talmi-Brody-Moshinshy methods 242 . (See Ref. 243 for details.) The results in Table 10 were obtained with approximately 100 Lanczos iterations: it is apparent that the convergence is then quite good. The wave functions must be normalized according to Eq. (205): this involves calculating unity as an effective operator. We will return to this point below.

The tables show the lovely evolution of the wave function in ET, an evolution quite unlike that of typical shell model calculations. The wave functions obtained in different model spaces agree over overlapping parts of their Hilbert spaces. Thus as one proceeds through $2 \hbar \omega, 4 \hbar \omega, 6 \hbar \omega, \ldots$ calculations, the ET wave function evolves only by adding new components in the expanded space. The normalization of the wave function grows accordingly. Thus, for ${ }^{3} \mathrm{He}$, the $0 \hbar \omega$ ET calculation contains 0.311 of the full wave function in the effective space; the $0+2+4 \hbar \omega$ result is 0.700 .

This evolution will not arise in the standard SM because the wave function normalization is set to unity regardless of the model space. It will also not arise for a second reason, illustrated in Table 11. The matrix elements of $H^{\text {eff }}$ are crucially dependent on the model space: the listed results for ${ }^{3} \mathrm{He}$ show that a typical matrix $\left\langle\alpha\left|H^{e f f}\right| \beta\right\rangle$ changes very rapidly under modest expansions of the model space, e.g., from $2 \hbar \omega$ to $4 \hbar \omega$. Yet it is common practice in the shell model to expand calculations by simply adding to an existing SM 
Hamiltonian new interactions that will mix in additional shells. We suspect the behavior found for ${ }^{3} \mathrm{He}$ is generic in ET calculations: it arises because a substantial fraction of the wave function lies near but outside the model space (e.g., see Table 10). An expansion of the model space changes the energy denominators for coupling to some of these configurations, and moves other nearby configurations from the excluded space to the model space. Naively, relative changes in effective interaction matrix elements of unity are expected.

Now we turn to the question of operators. The standard procedure in the $\mathrm{SM}$ is to calculate nuclear form factors with bare operators, or perhaps with bare operators renormalized according to effective charges determined phenomenologically at $q^{2}=0$, using SM wave functions normed to 1 . As we now have a series of exact effective interactions corresponding to different model spaces, we can test the validity of this approach. The results for the elastic magnetic form factors for the deuteron and ${ }^{3} \mathrm{He}$ are shown in Fig. 57. One sees
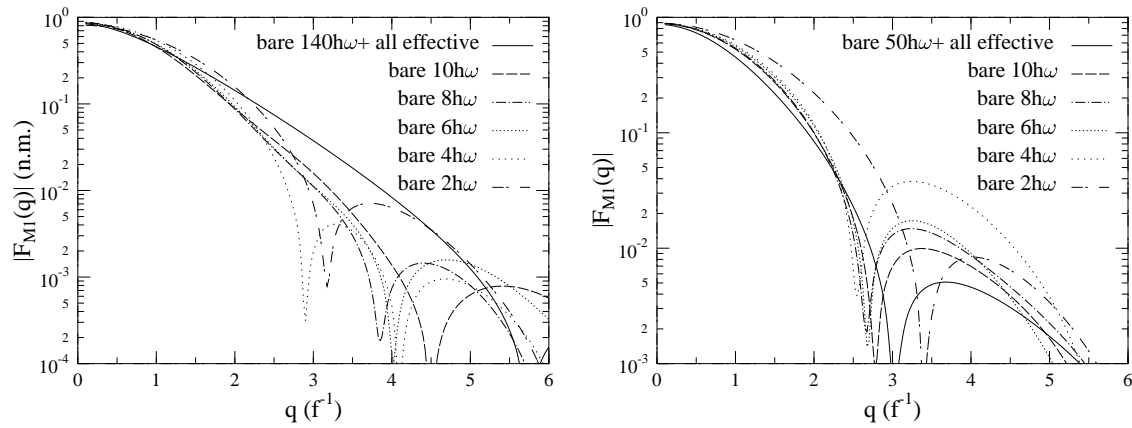

Figure 57: The magnetic elastic form factor for the deuteron (left panel) and ${ }^{3} \mathrm{He}$ (right panel) calculated with the exact $H^{e f f}$, SM wave funqtiopns pormalized to unity, and a bare operator are compared to the exact result (solid line) 225.226. When effective operators and the proper wave function normalizations are used, all results become identical to the solid line.

in each case that by the time one reaches a momentum transfer $q \sim 2.5 / f$, random numbers are being generated: bare operators used in conjunction with exact effective wave functions generate results that differ by an order of magnitude, depending on the choice of the model space. This is not surprising, of course. If one considers the operation

$$
e^{i \vec{q} \cdot \vec{r}} \sigma \tau_{3}|g . s .\rangle
$$

at momentum transfers $\gtrsim 2 k_{F}$, where $k_{F}$ is the Fermi momentum, most of the 
Table 10: As in Table 9, only for ${ }^{3} \mathrm{He}$. The basis states are now designated somewhat schematically as $|N, \alpha\rangle$, where $N$ is the total number of oscillator quanta and $\alpha$ is an index representing all other quantum numbers.

\begin{tabular}{|c|r|r|r|r|r|r|}
\hline \hline \multirow{3}{*}{ state } & \multicolumn{7}{|c|}{ amplitude } \\
\cline { 2 - 7 } & $0 \hbar \omega$ & $2 \hbar \omega$ & $4 \hbar \omega$ & $6 \hbar \omega$ & $8 \hbar \omega$ & exact \\
\cline { 2 - 7 } & $(31.1 \%)$ & $(57.4 \%)$ & $(70.0 \%)$ & $(79.8 \%)$ & $(85.5 \%)$ & $(100 \%)$ \\
\hline 0,1$\rangle$ & 0.55791 & 0.55791 & 0.55791 & 0.55795 & 0.55791 & 0.55793 \\
\hline 2,1$\rangle$ & 0.00000 & 0.04631 & 0.04613 & 0.04618 & 0.04622 & 0.04631 \\
\hline$|2,2\rangle$ & 0.00000 & -0.48255 & -0.48237 & -0.48243 & -0.48243 & -0.48257 \\
\hline$|2,3\rangle$ & 0.00000 & 0.00729 & 0.00731 & 0.00730 & 0.00729 & 0.00729 \\
\hline$|2,4\rangle$ & 0.00000 & 0.16707 & 0.16698 & 0.16706 & 0.16706 & 0.16708 \\
\hline$|2,5\rangle$ & 0.00000 & 0.00566 & 0.00564 & 0.00565 & 0.00565 & 0.00566 \\
\hline$|2,6\rangle$ & 0.00000 & -0.00017 & -0.00017 & -0.00017 & -0.00017 & -0.00017 \\
\hline$|4,1\rangle$ & 0.00000 & 0.00000 & -0.02040 & -0.02042 & -0.02043 & -0.02047 \\
\hline$|4,2\rangle$ & 0.00000 & 0.00000 & 0.11267 & 0.11274 & 0.11275 & 0.11289 \\
\hline$|4,3\rangle$ & 0.00000 & 0.00000 & -0.04191 & -0.04199 & -0.04208 & -0.04228 \\
\hline$|4,4\rangle$ & 0.00000 & 0.00000 & 0.28967 & 0.28978 & 0.28978 & 0.29001 \\
\hline$|4,5\rangle$ & 0.00000 & 0.00000 & 0.01059 & 0.01059 & 0.01059 & 0.01059 \\
\hline$|4,6\rangle$ & 0.00000 & 0.00000 & -0.00213 & -0.00212 & -0.00211 & -0.00210 \\
\hline$|4,7\rangle$ & 0.00000 & 0.00000 & 0.00998 & 0.01000 & 0.01000 & 0.01000 \\
\hline$|4,8\rangle$ & 0.00000 & 0.00000 & -0.11319 & -0.11327 & -0.11330 & -0.11335 \\
\hline$|4,9\rangle$ & 0.00000 & 0.00000 & 0.08446 & 0.08447 & 0.08446 & 0.08448 \\
\hline$|4,10\rangle$ & 0.00000 & 0.00000 & -0.08613 & -0.08626 & -0.08632 & -0.08638 \\
\hline$|4,11\rangle$ & 0.00000 & 0.00000 & -0.00210 & -0.00211 & -0.00211 & -0.00211 \\
\hline$|4,12\rangle$ & 0.00000 & 0.00000 & -0.00252 & -0.00254 & -0.00256 & -0.00257 \\
\hline$|4,13\rangle$ & 0.00000 & 0.00000 & -0.00020 & -0.00020 & -0.00020 & -0.00020 \\
\hline$|4,14\rangle$ & 0.00000 & 0.00000 & -0.00010 & -0.00010 & -0.00010 & -0.00010 \\
\hline$|4,15\rangle$ & 0.00000 & 0.00000 & -0.00012 & -0.00013 & -0.00012 & -0.00012 \\
\hline \hline
\end{tabular}

Table 11: Selected BH 3-body effective interaction matrix elements 225.226 for ${ }^{3} \mathrm{He}$, in $\mathrm{MeV}$, illustrating the strong dependence on the "SM" space. The Argonne V18 potential was used.

\begin{tabular}{|r|r|r|r|r|r|}
\hline \hline & $2 \hbar \omega$ & $4 \hbar \omega$ & $6 \hbar \omega$ & $8 \hbar \omega$ \\
\hline$\left\langle 0,1\left|H^{\text {eff }}\right| 2,1\right\rangle$ & -4.874 & -3.165 & -0.449 & 1.279 \\
\hline$\left\langle 0,1\left|H^{\text {eff }}\right| 2,5\right\rangle$ & -0.897 & -1.590 & -1.893 & -2.208 \\
\hline$\langle 2,1| H^{\text {eff }}$ & $2,2\rangle$ & 6.548 & -2.534 & -4.144 & -5.060 \\
\hline \hline
\end{tabular}


resulting amplitude should reside outside the long-wavelength model space, in any simple view of the nucleus. That is, the strength resides entirely in the effective contributions to the operator. If these components are ignored, the results have to be in error.

Clearly the effective interaction and effective operator have to be treated consistently and on the same footing. If $\hat{O}$ is the bare operator, one finds

$$
\left\langle\Psi_{f}|\hat{O}| \Psi_{i}\right\rangle \equiv\left\langle\Psi_{f}^{e f f}\left|\hat{O}^{e f f}\right| \Psi_{i}^{e f f}\right\rangle
$$

where

$$
\hat{O}^{e f f}=\left(1+H Q_{S M} \frac{1}{E_{f}-H Q_{S M}}\right) \hat{O}\left(1+\frac{1}{E_{i}-Q_{S M} H} Q_{S M} H\right)
$$

and where the effective wave function normalization of $\left|\Psi_{i}^{e f f}\right\rangle$ and $\left|\Psi_{f}^{e f f}\right\rangle$, mentioned earlier, must be determined using the effective operator $\hat{1}$, e.g.,

$1=\left\langle\Psi_{i} \mid \Psi_{i}\right\rangle=\left\langle\Psi_{i}^{e f f}\left|\left(1+H Q_{S M} \frac{1}{E_{i}-H Q_{S M}}\right)\left(1+\frac{1}{E_{i}-Q_{S M} H} Q_{S M} H\right)\right| \Psi_{i}^{e f f}\right\rangle$

These expressions can be evaluated with the Lanczos Green's function methods described earlier. When this is done, all of the effective calculations, regardless of the choice of the model space, yield the same result, given by the solid lines in Fig. 57.

We would argue, based on this example, that many persistent problems in nuclear physics - ranging from the renormalization of $g_{A}$ in $\beta$ decay to the systematic differences between measured and calculated M1 electromagnetic form factors - very likely are due to naive treatments of operators, treatments that fail to satisfy the basic rules of ETs. It should be apparent from the above example that no amount of work on $H^{e f f}$ will help with this problem. What is necessary is a diagrammatic basis for generating $H^{e f f}$ that can be applied in exactly the same way to evolving $\hat{O}^{e f f}$. From this perspective, phenomenological derivations of $H^{e f f}$ by fitting binding energies and other static properties of nuclei are not terribly helpful, unless one intends to simultaneously find phenomenological renormalizations for each desired operator in each $q^{2}$ range of interest.

The point of this discussion has been largely pedagogical. To our knowledge the examples given above are the only ones in the classical nuclear physics literature in which a "model space" calculation has been formulated in a way that satisfies the basic rules of ET. The results - particularly the invariance of energies and operator matrix elements under changes in how we define $Q$ - are obvious from the perspective of ET. The fact that standard techniques 
like the SM violate so many of the rules of ETs is cause for optimism: much can be done to improve the rigor of such nuclear physics tools. As we have demonstrated here, new numerical methods can be developed to handle the full ET problem at the cost of only modest additional effort.

\subsection{Crossing the Many-Body Border}

The effort to derive SM effective interactions directly from realistic NN interactions was largely abandoned in the early 1970's when it became apparent that the high-momentum scattering problem was nonperturbive. A series of efforts involving perturbative expansions in either the potential $V$ or the corresponding $G$-matrix (the sum of the two-body ladder diagrams) yielded discouraging results, with higher-order terms often dominating over lower-order one 44 . In particular Shucan and Weidenmuller showed that any overlap in the spectra of included and excluded, high-momentum states would lead to a nonperturbative serie 239.240. Thus, the uncontrolled approximations in the SM approach grew out of necessity: it became a largely phenomenological tool in part because early efforts to provide a more rigorous basis for the model ended in failure.

Given our two SM-like ET calculations, the deuteron and ${ }^{3} \mathrm{He}$, it is interesting to return to this old problem of the nonperturbative behavior of the nonrelativistic many-body problem, using these examples as laboratories. Today's challenge, in fact, is more modest than that of the 1970's: modern computers coupled with new algorithms, like the Lanczos solution of the BH equation introduced above, allow us to solve rather complex nonperturbative problems. To extend the calculations above to ${ }^{4} \mathrm{He}$ and heavier nuclei one has to achieve only modest increases in efficiency, such as a substantial loyering of $\Lambda_{\infty}$. Below we summarize some very recent work by Haxton and Lut245 that suggests that the goals of the 1970's - a perturbative treatment of the nonrelativistic nuclear physics problem - may not be out of the question. Through renormalization group and other techniques, the most severely nonperturbative contributions to the potential can be systematically extracted and treated. The approach is quite similar to that of EFTs. We conclude our discussion of the many-body problem by sketching this effort to cross the border between EFTs and conventional nuclear models.

The deuteron calculations were performed for $\Lambda_{\infty} \sim 140$, a value where the hard core of the AV18 potential is resolved sufficiently well that the resulting binding energy is good to $1 \mathrm{keV}$. Results are variational in this parameter: as $\Lambda_{\infty}$ is increased, the correct binding energy is approached from above. The evolution was explored in Ref.225 and found to vary as

$$
E_{g s} \sim-2.175 \mathrm{MeV}+3.178 \mathrm{MeVe}^{-1.055\left(\Lambda_{\infty} / 50\right)^{2}}
$$


to very good accuracy, a functional dependence that already suggests some nonperturbative behavior.

The BH calculation determines the full set of matrix elements $\left\langle\alpha\left|H^{e f f}\right| \beta\right\rangle$ for the SM space. These individual matrix elements carry information about the convergence not easily seen in $E_{g s}$. Their behavior in perturbation theory can be examined by expanding the $\mathrm{BH}$ propagator

$$
\frac{1}{E-Q H}=\frac{1}{E-H_{0}}+\frac{1}{E-H_{0}} Q\left(V-V_{0}\right) \frac{1}{E-H_{0}}+\cdots,
$$

evaluating the series term by term. Here $H_{0}$ and $V_{0}$ are the harmonic oscillator Hamiltonian and potential. We thus lower $\Lambda_{\infty}$ and test whether states above $\Lambda_{\infty}$ can be treated perturbatively.

Once a scale of $\Lambda_{\infty} \sim 70$ is reached, striking differences appear in the rate of convergence of different matrix elements. All are greatly improved in first and second order perturbation theory. Those matrix elements well away from the "boundary" at $\Lambda_{\infty}$ converge quickly to their correct values in this way. However, those matrix elements $\left\langle\alpha\left|H^{e f f}\right| \beta\right\rangle$ where either $|\alpha\rangle$ or $|\beta\rangle$ resides in the last shell (with $N=\Lambda_{\infty}$ ) converge only very slowly to the correct values, after the initial improvement in low-order perturbation theory. Typical $\sim 10^{3}$ orders of perturbation theory are required to produce the correct value. This clearly suggests that slow convergence is associated with the relative kinetic energy operator $Q T$ contribution to $Q H$, as the only transitions to states outside the Hilbert space generated by this operator have $\Delta n=1$. At large $r$ the strength of this transition becomes quite large, $-Q V_{0}(r)|\alpha\rangle$, reflecting the unphysical asymptotic behavior of harmonic oscillator wave functions. This amplitude propagates nonperturbatively as $\left(V-V_{0}\right) / H_{0} \sim 1$. Eventually enough high-momentum harmonic oscillator wave functions are coupled together to produce the softer asymptotic fall-off characteristic of the correct bound-state wave function

As discussed in Ref. 245, the required resummation is guided by the observation that the true potential, $V(r)$, falls off properly at large $r$. Thus a reorganization of the $\mathrm{BH}$ equation in which the propagator is always sandwiched between $V(r)$ should remove the unwanted propagation. This leads to the following recasting of the $\mathrm{BH}$ equation:

$$
\begin{aligned}
\left\langle\alpha\left|H^{e f f}\right| \beta\right\rangle= & \langle\alpha|T| \beta\rangle+(\langle\hat{\alpha}|-\langle\alpha|) E-T(|\hat{\beta}\rangle-|\beta\rangle) \\
& +\left\langle\hat{\alpha}\left|V+V \frac{1}{E-Q H} Q V\right| \hat{\beta}\right\rangle
\end{aligned}
$$

where

$$
|\hat{\alpha}\rangle=\frac{E}{E-Q T}|\alpha\rangle
$$


If $\alpha$ and $\beta$ are not in the last shell, $|\hat{\alpha}\rangle=|\alpha\rangle$ and $|\hat{\beta}\rangle=|\beta\rangle$, so that the above rewriting of the $\mathrm{BH}$ equation reduces to the original form. Otherwise, a modified wave function generated by Eq. (217) must be used.

This discussion is a necessary diversion from the principal topic of this section: this source of nonperturbative behavior dominates one that is of more interest (and more difficult to remove), hard core scattering. It should be clear the the lowering of the scale $\Lambda_{\infty}$ at the cost of an evaluation of Eq. (217) represents a tremendous savings: in place of a dense matrix $Q H$ whose elements have to evaluated numerically, we have a sparse matrix $Q T$ whose matrix elements are known analytically. The kinetic energy Green's function is thus trivial to evaluate via Eq. (208). For example, in the case of the deuteron, $Q T$ is tridiagonal in the harmonic oscillator basis. Thus the Green's function can be expressed analytically in the form of Eq. (208).

Once the replacement $|\alpha\rangle \rightarrow|\hat{\alpha}\rangle$ is made, $\Lambda_{\infty}$ can be lowered to $\sim 40$ while maintaining $1 \mathrm{keV}$ accuracy, if one works to third order in perturbation theory. But with further lowering, errors arise in the perturbative expansion that persists even if calculations are carried to very high order. At $\Lambda_{\infty}=30$, third order perturbation theory reduces $\sim 10 \%$ errors in $\left\langle n^{\prime}=1 l^{\prime}=0\left|H^{\text {eff }}\right| n=1 l=0\right\rangle$ to $\sim 0.2 \%$. But an error in excess of $0.1 \%$ - corresponding to $50 \mathrm{keV}$ persists after 10 additional orders of perturbation. Numerically one can verify that the nonperturbative tail is generated by the scattering at very small $r$. As expected, the nonperturbative contributions to $s-d$ matrix elements are much smaller than those for $\left\langle n^{\prime}=1 l^{\prime}=0\left|H^{e f f}\right| n=1 l=0\right\rangle$ and other $s-s$ matrix elements.

We sketch an elegant solution to this problem described in Ref.245. The most singular short-ranged contributions are removed from the potential analytically through a renormalization group (RG) procedure. The method starts with the well-known Talmi integral expansion of harmonic oscillator radial matrix elements and involves a systematic removal of the most singular of these by introducing local, separable operators familiar from EFT treatments. The coefficients of these operators are then run by the RG equations. The result establishes a very close connection between potential descriptions and EFT treatments, and also allows a clean separation of long-distance contributions to the potential that can be treated perturbatively.

As our goal here is to illustrate the procedure, we work to LO. (The NLO and $\mathrm{N}^{2} \mathrm{LO}$ results are given in Ref. 245.) One begins with the Talmi integral expansion for radial harmonic oscillator matrix elements

$$
\left\langle n^{\prime} l^{\prime}\left|V_{s r}(r)\right| n l\right\rangle=\int_{0}^{\infty} R_{n^{\prime} l^{\prime}}(r) V_{s r}(r) R_{n l}(r) r^{2} d r
$$




$$
=\sum_{p} B\left(n^{\prime}, l^{\prime} ; n, l ; p\right) I_{p}(b)
$$

where

$$
I_{p}(b)=\frac{2}{\Gamma(p+3 / 2)} \int_{0}^{\infty} e^{-r^{2} / b^{2}} V_{s r}(r) \frac{r^{2 p+2} d r}{b^{2 p+3}} .
$$

The $B\left(n^{\prime}, l^{\prime} ; n, l ; p\right)$ are known analytically 24 . Here $V_{s r}(r)$ denotes the shortrange contributions to the potential (we return to this point below). The integrals $I_{p}$ can be viewed as a systematic expansion of the nonperturbative hard core in terms of the parameter $\left(r_{c} / b\right)^{2}$, where $r_{c}$ is a distance associated with the size of the hard core that remains unresolved at scale $\Lambda_{\infty}$. Thus the LO term in the expansion is

$$
I_{p=0}^{(0)}=\frac{2}{\Gamma(3 / 2)} \frac{1}{b^{3}} \int_{0}^{\infty} V_{s r}(r) r^{2} d r
$$

while in NLO one includes

$$
\begin{aligned}
I_{p=0}^{(1)} & =\frac{2}{\Gamma(3 / 2)} \frac{1}{b^{3}} \int_{0}^{\infty} V_{s r}(r) r^{2}\left(1-\frac{r^{2}}{b^{2}}\right) d r \\
I_{p=1}^{(0)} & =\frac{2}{\Gamma(5 / 2)} \frac{1}{b^{5}} \int_{0}^{\infty} V_{s r}(r) r^{4} d r .
\end{aligned}
$$

In $\mathrm{N}^{2} \mathrm{LO}$ one obtains

$$
\begin{aligned}
I_{p=0}^{(2)} & =\frac{2}{\Gamma(3 / 2)} \frac{1}{b^{3}} \int_{0}^{\infty} V_{s r}(r) r^{2}\left(1-\frac{r^{2}}{b^{2}}+\frac{r^{4}}{2 b^{4}}\right) d r \\
I_{p=1}^{(1)} & =\frac{2}{\Gamma(5 / 2)} \frac{1}{b^{5}} \int_{0}^{\infty} V_{s r}(r) r^{4}\left(1-\frac{r^{2}}{b^{2}}\right) d r \\
I_{p=2}^{(0)} & =\frac{2}{\Gamma(7 / 2)} \frac{1}{b^{7}} \int_{0}^{\infty} V_{s r}(r) r^{6} d r .
\end{aligned}
$$

and so on.

Now the radial component of the $s$-wave potential $V_{0} \delta(\mathbf{r})$ is

$$
V_{\delta}^{(0)}(r)=V_{0} \frac{1}{4 \pi r^{2}} \delta(r) .
$$

Substituting this into Eq. (218) yields

$$
\left\langle n^{\prime} l^{\prime}=0\left|V_{\delta}^{(0)}(r)\right| n l=0\right\rangle=B\left(n^{\prime}, 0 ; n, 0 ; 0\right) I_{0}^{\delta}(b)
$$


where

$$
I_{0}^{\delta}(b)=\frac{2}{\Gamma(3 / 2)} \frac{1}{4 \pi b^{3}} V_{0}
$$

It follows that the contact interaction will produce the exact lowest-order Talmi integral contribution (Eq. (220) ) to $V_{s r}$ provided

$$
V_{0}=4 \pi \int_{0}^{\infty} V_{s r}(r) r^{2} d r
$$

Using a normalization that will be convenient later, we can then rewrite $V_{s r}(\mathbf{r})$ as

$$
V_{s r}(\mathbf{r})=V_{s r}^{(1)}(\mathbf{r})+b^{3} \frac{\pi^{2}}{2} \hbar \omega a_{0}^{s s}\left(\Lambda_{\infty}\right) \delta(\mathbf{r})
$$

where $V_{s r}^{(1)}$ is a new potential whose leading-order behavior is of order $\left(r_{c} / b\right)^{2}$ relative to $V_{s r}$ (determined by subtracting from the original Talmi integral expression for $V_{s r}$ the contribution from Eq. (220)). The coefficient $a_{0}^{s s}\left(\Lambda_{\infty}\right)$ is a dimensionless coupling (Note that throughout this discussion, we have suppressed the spin and isospin of $V_{s r}(\mathbf{r})$. Thus $V_{s r}(r)$ represents the radial function obtained after spin and isospin matrix elements have been taken. For example, the components of the AV18 potential contributing to $s-s$ transitions is

$$
V_{s r}(\mathbf{r})=V_{1}(r)+V_{2}(r) \vec{\tau}_{1} \cdot \vec{\tau}_{2}+V_{3}(r) \vec{\sigma}_{1} \cdot \vec{\sigma}_{2}+V_{4}(r) \vec{\sigma}_{1} \cdot \vec{\sigma}_{2} \vec{\tau}_{1} \cdot \vec{\tau}_{2}
$$

so that

$$
V_{s r}(r)=V_{1}(r)-3 V_{2}(r)+V_{3}(r)-3 V_{4}(r)
$$

for the deuteron $(l=0, s=1, t=0)$.

The above procedure is, in fact, general. The NLO contribution (Eq. (221)) can be removed from $s-s$ wave deuteron matrix elements of $V_{s r}(\mathbf{r})$ by introducing a second, higher-order contact operator

$$
a_{2}^{s s}\left(\Lambda_{\infty}\right) \frac{1}{2}\left(\overleftarrow{\nabla}^{2} \delta(\mathbf{r})+\delta(\mathbf{r}) \vec{\nabla}^{2}\right)
$$

where

$$
a_{2}^{s s}\left(\Lambda_{\infty}\right)=\frac{8}{3 \pi b^{5} \hbar \omega} \int_{0}^{\infty} V_{s r}(r) r^{4} d r
$$

while the $\mathrm{N}^{2} \mathrm{LO}$ contributions can be removed by

$$
a_{4}^{s s}\left(\Lambda_{\infty}\right)\left(\overleftarrow{\nabla}^{2} \delta(\mathbf{r}) \vec{\nabla}^{2}+\frac{3}{10}\left(\overleftarrow{\nabla}^{4} \delta(\mathbf{r})+\delta(\mathbf{r}) \vec{\nabla}^{4}\right)\right)
$$


where

$$
a_{4}^{s s}\left(\Lambda_{\infty}\right)=\frac{2}{9 \pi b^{7} \hbar \omega} \int_{0}^{\infty} V_{s r}(r) r^{6} d r .
$$

Likewise there are coeficients of additional contact operators (denoted $a_{2}^{s d}, a_{4}^{s d}$, and $a_{4}^{d d}$ in Ref.245) that remove the Talmi integral contributions to $s-d$ and $d-d$ matrix elements to $\mathrm{N}^{2} \mathrm{LO}$.

Up to this point we have simply rewritten the "bare" potential — the potential that acts in the space defined by $\Lambda_{\infty}$ - in an entirely equivalent form, exploiting the Talmi integral expansion and the fact that the unique translationally-invariant two-body contact operators generate those Talmi integrals. Now we consider, in LO, the effects on $a_{0}^{s s}$ of integrating out the single shell at $\Lambda_{\infty}$, thereby mapping the original problem into an effective one with $\Lambda_{\infty} \rightarrow \Lambda_{\infty}-2$. We require that some low-energy matrix element

$$
\left\langle n^{\prime} 0\left|V_{s r}(\mathbf{r})\right| n 0\right\rangle
$$

remain invariant, so that $a_{0}^{s s}(\Lambda)$ must run. The relevant equation for $a_{0}^{s s}$ then is the single-shell limit of the $\mathrm{BH}$ equation, evaluated in LO (which eliminates $s d$ and $d d$ contributions to transitions and the Green's function). The result is 245

$$
a_{0}^{s s}(\Lambda-2)=a_{0}^{s s}(\Lambda)+\frac{\Gamma\left(\frac{\Lambda+3}{2}\right)}{\left(\frac{\Lambda}{2}\right) !} \frac{a_{0}^{s s}(\Lambda)^{2}}{E_{0}-\left(\Lambda+\frac{3}{2}\right)-a_{0}^{s s}(\Lambda) \frac{\Gamma\left(\frac{\Lambda+3}{2}\right)}{\left(\frac{\Lambda}{2}\right) !}}
$$

where $E_{0}=E / \hbar \omega$ is a dimensionless energy. ( $E$ is the $\mathrm{BH}$ energy being determined selfconsistently.) This leading-order RG equation is independent of the matrix element chosen to evaluate the running: it is an operator equation, independent of the running scheme. Thus, to this order, the most singular Talmi integral can be subtracted exactly from all matrix elements in the lowmomentum space. This difference equation thus tells us how to run $a_{0}^{s s}\left(\Lambda_{\infty}\right)$ to any desired new scale $\Lambda$.

While the RG equation is a difference equation, the connections with EFT become even clearer by introducing the natural definition of the derivative

$$
\frac{d a_{0}^{s s}(\Lambda)}{d \Lambda} \equiv \frac{a_{0}^{s s}(\Lambda)-a_{0}^{s s}(\Lambda-2)}{2} .
$$

As will be apparent from results given below, the terms in the denominator of Eq. (235) that compete with $\Lambda$ are one power lower in $\Lambda$. Thus for large $\Lambda$ the $\mathrm{RG}$ equation can be written in a more conventional form

$$
\Lambda \frac{d a_{0}^{s s}(\Lambda)}{d \Lambda} \sim \frac{1}{2} \sqrt{\frac{\Lambda}{2}} a_{0}^{s s}(\Lambda)^{2}
$$


which has the solution

$$
a_{0}^{s s}(\Lambda) \sim-\sqrt{\frac{2}{\Lambda}} .
$$

As $\Lambda$ is a dimensionless nonrelativistic energy, this is equivalent to running as $1 / p$, where $p$ is the momentum, a result familiar from EFT. Similarly, $a_{2}^{s s}$ runs as $\Lambda^{-3 / 2}$ and $a_{4}^{s s}$ as $\Lambda^{-5 / 2}$.

This procedure is followed through order $(r / b)^{4}$ in Ref. 245, which involves a series of coupled RG equations for $a_{0}^{s s}, a_{2}^{s s}, a_{4}^{s s}, a_{2}^{s d}, a_{4}^{s d}$, and $a_{4}^{d d}$. Beyqnd lowest order the running is no longer scheme independent; the fit in Ref. 245 is to the longest wavelength properties of the low-energy system, the set of matrix elements coupling the $n=1$ and $n=2$ oscillator shells. Thus the analogy with standard EFT approaches is very close. In effect, the primary difference is that in EFTs the strengths of the local operators are determined by directly fitting data. Here a phenomenological potential - itself fit to data - determines the initial value of the local operators that are then run by the RG equation. It is also apparent that the detailed short-range form of the potential is irrelevant to shell-model-inspired effective theories: the needed quantities are Talmi integrals over the short-range part of the potential. Any change in the detailed radial behavior of the potential that preserves the value of the Talmi integrals will also leave the low-energy theory unchanged.

The numerical results in Ref. 245 are quite promising: in $\mathrm{N}^{2} \mathrm{LO}$, the hard core (below $0.7 \mathrm{f}$ ) nonperturbative contribution to the scattering can be stepped down to $\Lambda \sim 10$ - that is, to nearly a typical SM scale — with a loss of accuracy of $\sim 7 \mathrm{keV}$. The portion of the core one can treat accurately increases as the order of the RG calculation is increased. As the potential is known, one can - if one is willing to invest sufficient effort - carry this procedure out to an arbitrary number of orders. The numerical results are given in Fig. 58. One sees that the accuracy with which one can run to small $\Lambda$ continues to improve according to the work invested (LO, NLO, and $\mathrm{N}^{2} \mathrm{LO}$ ) in the RG equations.

The detailed use of this Talmi integral subtraction and related RG procedures will not be discussed here, though it is treated in Ref.245. The essential idea is to exploit the RG equations to treat the nonperturbative scattering due to the hard core, then use perturbation theory to handle the remaining softer parts of the potential. Thus $V_{s r}(r)$ is defined in terms of the Talmi integrals for $V(r) \theta\left(r_{c}-r\right)$, where $r_{c}$ is the radius of the unresolved hard core. The marriage of perturbation theory and RG techniques can be done in a number of ways: many aspects of this question remain to be explored. However it is clear these two tools are complementary. If one starts at very high $\Lambda_{\infty}$, the part of the hard core that cannot be treated by perturbation theory is limit to a very small region near $r \sim 0$. However to minimize the effects of omitted higher 


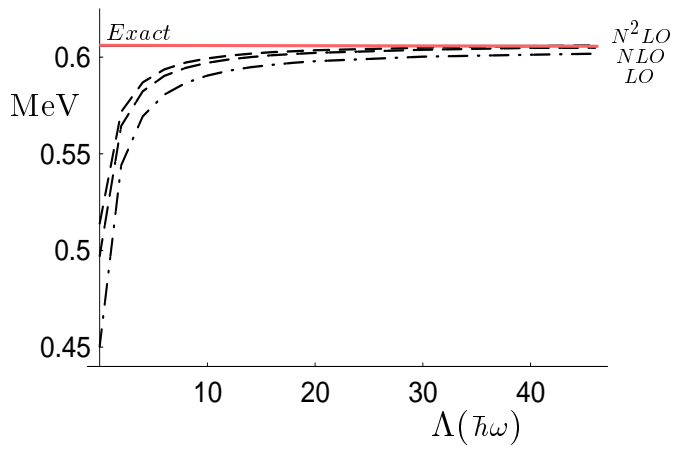

Figure 58: The results of integrating out the hard-core contribution $\left(r_{c}=0.7 \mathrm{f}\right)$ to the AV18 potential by the RG method, in LO, NLO, and $\mathrm{N}^{2} \mathrm{LO}$. These orders correspond to the removal of successively less less singular contributions of the leading-order Talmi integrals, and thus can be viewed as an expansion in $\left(r_{c} / b\right)^{2}$. One can lower the scale of the low-energy theory from $\Lambda_{\infty}=50$ to 10 analytically with the loss of $\sim 7 \mathrm{keV}$ in the accuracy of the deuteron ground-state energy in $\mathrm{N}^{2} \mathrm{LO}$.

order terms (i.e., Talmi integrals) in the RG equation for matrix elements with large values of $n, r_{c}$ must be made quite small. As $\Lambda_{\infty}$ is lowered, $r_{c}$ can be extended without loss of accuracy, but perturbation theory - an expansion in $1 / \Lambda$ - becomes less effective.

The extensions to three- and four-body clusters have not yet been carried out, though nothing in the procedures is specific to the deuteron. The RG equations will, of course, involve the full set of general three- and four-body translationally invariant contact operators that can be constructed, just as EFT treatments of the three-body problem were required to introduce threebody contact terms. Thus the RG equations become more complicated, but not conceptually more difficult.

\section{Conclusion}

In this chapter we have given a somewhat detailed overview of the progress that has been made in applying the ideas of EFT to nuclear physics. This will ultimately provide the bridge between QCD and the rigorous study of multi-nucleon processes. The field is still somewhat embryonic, but as we have shown, impressive (and rigorous) results have already been obtained. There is much more to come! 


\section{Acknowledgments}

We are grateful to our very learned colleagues who are responsible for much of our lack of understanding of this field. We would like to thank Harald Grießhammer, Tom Lu, and all our collaborators. This work is supported in part by the U.S. Dept. of Energy under Grants No. DE-FG03-97ER014 (NT@UW-00-021) (S.B., D.P. and M.S.) and DOE-ER-41132-101 (P.B. and W.H.).

\section{References}

1. J. Carlson and R. Schiavilla, Rev. Mod. Phys. 70, 743 (1998).

2. V. G. J. Stoks, R. A. M. Klomp, M. C. M. Rentmeester, and J. J. de Swart, Phys. Rev. C 48, 792 (1993).

3. H. Yukawa, Proc. Phys. Math. Soc. Jpn. 17, 48 (1935).

4. V. G. J. Stoks, R. A. M. Klomp, C. P. F. Terheggen, and J. J. de Swart, Phys. Rev. C 49, 2950 (1994).

5. R. B. Wiringa, V. G. J. Stoks, and R. Schiavilla, Phys. Rev. C 51, 38 (1995), nucl-th/9408016.

6. R. Machleidt, hucl-th/0006014.

7. S. Weinberg, Phys. Lett. B251, 288 (1990).

8. S. Weinberg, Nucl. Phys. B363, 3 (1991).

9. S. Weinberg, Phys. Lett. B295, 114 (1992).

10. G. P. Lepage, nucl-th/9706029.

11. H. A. Bethe, Phys. Rev. 76, 38 (1949).

12. H. A. Bethe and C. Longmire, Phys. Rev. 77, 647 (1950).

13. A. V. Manohar and H. Georgi, Nucl. Phys. B234, 189 (1984).

14. E. P. Wigner, Phys. Rev. 98, 145 (1955).

15. D. R. Phillips and T. D. Cohen, Phys. Lett. B390, 7 (1997), nuclth/9607048.

16. J. J. de Swart, C. P. F. Terheggen, and V. G. J. Stoks, nucl-th/9509032.

17. D. B. Kaplan, Nucl. Phys. B494, 471 (1997), nucl-th/9610052.

18. D. B. Kaplan, M. J. Savage, and M. B. Wise, Nucl. Phys. B478, 629 (1996), nucl-th/9605002.

19. T. D. Cohen, J. L. Friar, G. A. Miller, and U. van Kolck, Phys. Rev. C 53, 2661 (1996), nucl-th/9512036.

20. T.-S. Park, K. Kubodera, D.-P. Min, and M. Rho, Phys. Rev. C 58, 637 (1998), hep-ph/9711463.

21. S. R. Beane, T. D. Cohen, and D. R. Phillips, Nucl. Phys. A632, 445 (1998), nucl-th/9709062. 
22. S. K. Adhikari and A. Ghosh, J. Phys. A 30, 6553 (1997), hepth/9706193.

23. D. R. Phillips, S. R. Beane, and T. D. Cohen, Ann. Phys. (N.Y.) 263, 255 (1998), hep-th/9706070.

24. J. V. Steele and R. J. Furnstahl, Nucl. Phys. A637, 46 (1998), nuclth/9802069.

25. D. R. Phillips, in Nuclear physics with effective field theory. Proceedings, Joint Caltech/INT Workshop, Pasadena, USA, February 26-27, 1998, , (World Scientific, Singapore, 1998), nucl-th/9804040.

26. A. Ghosh, S. K. Adhikari, and B. Talukdar, Phys. Rev. C58, 1913 (1998), nucl-th/9810012.

27. J. Gegelia, J. Phys. G 25, 1681, nucl-th/9805008.

28. M. Lutz, p. 22 (1996), hep-ph/9606301.

29. U. van Kolck, in Mainz 1997, Chiral Dynamics: Theory and Experiment, ed. A. M. Bernstein, D. Drechsel, and T. Walcher, (Springer-Verlag, Berlin, 1998).

30. D. B. Kaplan, M. J. Savage, and M. B. Wise, Nucl. Phys. B534, 329 (1998), nucl-th/9802075.

31. D. B. Kaplan, M. J. Savage, and M. B. Wise, Phys. Lett. B424, 390 (1998), nucl-th/9801034.

32. U. van Kolck, Nucl. Phys. A645, 273 (1999), nucl-th/9808007.

33. D. R. Phillips, G. Rupak, and M. J. Savage, Phys. Lett. B473, 209 (2000), nucl-th/9908054.

34. J. Gegelia, Phys. Lett. B429, 227 (1998).

35. T. Mehen and I. W. Stewart, Phys. Lett. B445, 378, nucl-th/9809071.

36. T. Mehen, I. W. Stewart, and M. B. Wise, Phys. Lett. B474, 145 (2000), hep-th/9910025.

37. M. C. Birse, J. A. McGovern, and K. G. Richardson, PiN Newslett. 15, 280 (1999), nucl-th/9911048.

38. P. Bedaque and U. van Kolck, Phys. Lett. B428, 221, nucl-th/9710073.

39. J.-W. Chen, G. Rupak, and M. J. Savage, Nucl. Phys. A653, 386 (1999), nucl-th/9902056.

40. J.-W. Chen, G. Rupak, and M. J. Savage, Phys. Lett. B464, 1 (1999), nucl-th/9905002.

41. G. Rupak, (1999), nucl-th/9911018.

42. H. P. Stapp, T. J. Ypsilantis, and N. Metropolis, Phys. Rev. 105, 302 (1957).

43. J. J. de Swart, C. P. F. Terheggen, and V. G. J. Stoks, (1995), nuclth/9509032.

44. S. Fleming, T. Mehen, and I. W. Stewart, Phys. Rev. C61, 044005 
(2000), nucl-th/9906056.

45. D. M. Bishop and L. M. Cheung, Phys. Rev. C 20, 381 (1979).

46. T. E. O. Ericson and M. Rosa-Clot, Nucl. Phys. A405, 497 (1983).

47. H. G. J. Adam Jr. and H. Arenhovel, Phys. Rev. C48, 370 (1993).

48. C. W. Wong, Int. J. of. Mod. Phys. E3, 821 (1994).

49. H. H. A.J. Buchmann and P. Sauer, Few-Body Systems 21, 149 (1996).

50. J. M. J. L. Friar and D. W. L. Sprung, Phys. Rev. A56, 4579 (1997).

51. T. Ericson and W. Weise, (Oxford University Press, 1988).

52. D. B. Kaplan, M. J. Savage, and M. B. Wise, Phys. Rev. C 59, 617 (1999), nucl-th/9804032

53. D. Drechsel et al., (1997), nucl-th/9712013.

54. J. L. Friar and G. L. Payne, Phys. Rev. C55, 2764 (1997), nuclth/9702019.

55. M. I. Levchuk and A. I. Lvov, Few Body Syst. Suppl. 9, 439 (1995).

56. P. T. Wilbois and H. Arenhovel, Few Body Syst. Suppl. 9, 263 (1995).

57. A. Karchenko, Ukr. J. of Phys. 42, 18 (1997).

58. J.-W. Chen and M. J. Savage, Phys. Rev. C60, 065205 (1999), nuclth/9907042.

59. T. M. Muller, Private Communication (2000).

60. H. Austern, Phys. Rev. 92, 670 (1953).

61. A.E. Cox, S.A.R. Wynchank, and C. Collie, Nucl. Phys. 74, 497 (1965).

62. D. O. Riska and G. E. Brown, Phys. Lett. 38B, 193 (1972).

63. T.-S. Park, D.-P. Min, and M. Rho, Phys. Rev. Lett. 74, 4153 (1995), nucl-th/9412025.

64. T.-S. Park, D.-P. Min, and M. Rho, Nucl. Phys. A596, 515 (1996), nucl-th/9505017.

65. W. Leidemann, Private Communication (2000).

66. A. N. Bazhenov et al., Phys. Letts. B289, 17 (1992).

67. A. P. Burichenko and I. B. Khriplovich, Nucl. Phys. A515, 139 (1990).

68. T.-S. Park, K. Kubodera, D.-P. Min, and M. Rho, Phys. Lett. B472, 232 (2000), nucl-th/9906005.

69. S. D. Ellis and J. N. Bahcall, Nucl. Phys. A114, 636 (1968).

70. J. N. Bahcall and R. M. May, Ap. J 55, 501 (1969).

71. R. Schiavilla et al., Phys. Rev. C58, 1263 (1998), nucl-th/9808010.

72. J. N. Bahcall, K. Kubodera, and S. Nozawa, Phys. Rev. D38, 1030 (1988).

73. N. Tatara, Y. Kohyama, and K. Kubodera, Phys. Rev. C42, 1694 (1990).

74. M. Doi and K. Kubodera, Phys. Rev. C45, 1988 (1992).

75. K. Kubodera and S. Nozawa, USC(NT)-report-92-1 (unpublished) 
(1992).

76. K. Kubodera and S. Nozawa, Int. J. Mod. Phys. E3, 101 (1994), nucl-th/9310014.

77. S. Ying, W. Haxton, and E. M. Henley, Phys. Rev. D40, 3211 (1989).

78. S. Ying, W. C. Haxton, and E. M. Henley, Phys. Rev. C45, 1982 (1992).

79. V. G. S. Nakamura, T. Sato and K. Kubodera, private communication (2000).

80. X. Kong and F. Ravndal, Nucl. Phys. A656, 421 (1999), nuclth/9902064.

81. X. Kong and F. Ravndal, Nucl. Phys. A665, 137 (2000), hep$\mathrm{ph} / 9903523$.

82. X. Kong and F. Ravndal, Phys. Lett. B470, 1 (1999), nucl-th/9904066.

83. X. Kong and F. Ravndal, (2000), nucl-th/0004038.

84. T.-S. Park, K. Kubodera, D.-P. Min, and M. Rho, Astrophys. J. 507, 443 (1998), astro-ph/9804144.

85. M. Butler and J.-W. Chen, Nucl. Phys. A675, 575 (2000), nuclth/9905059.

86. M. Butler, J.-W. Chen, and X. Kong, (2000), nucl-th/0008032.

87. M. A. Lucas, PhD thesis, University of Illinois, 1994, unpublished.

88. P. F. Bedaque, H. W. Hammer, and U. van Kolck, Phys. Rev. C 58, R641 (1998), nucl-th/9802057.

89. F. Gabbiani, P. F. Bedaque, and H. W. Griesshammer, Nucl. Phys. A675, 601 (2000), nucl-th/9911034.

90. G. V. Skornyakov and K. A. Ter-Martirosian, Sov. Phys. JETP 4, 648 (1957).

91. J. H. Hetherington and L. H. Schick, Phys. Rev. B 137, 935 (1965).

92. R. T. Cahill and I. H. Sloan, Nucl. Phys. 165, 161 (1971).

93. R. Aaron and R. D. Amado, Phys. Rev. 150, 857 (1966).

94. V. Efimov, Phys. Rev. 44, 2303 (1991).

95. G. S. Danilov, Sov. Phys. JETP 16, 1010 (1963).

96. R. A. Minlos and L. D. Fadeev, Sov. Phys. JETP 14, 1315 (1962).

97. G. S. Danilov, Phys. JETP. 16, 110 (1963).

98. E. Huttel, W. Arnold, H. Baumgart, and G. Clausnitzer, Nucl. Phys. A 406, 443 (1983).

99. P. A. Schmelzbach et al., Phys. A 197, 273 (1972).

100. A. Kievsky, S. Rosati, W. Tornow, and M. Viviani, Nucl. Phys. A 607, $402(1996)$

101. A. Kievsky, private communication .

102. W. Dilg, L. Koester, and W. Nistler, Phys. Lett. B 36, 208 (1971).

103. J. L. Friar, D. Huber, H. Witala, and G. L. Payne, Acta Phys. Polon. 
B 31, 749 (2000), nucl-th/9908058.

104. V. Efimov, Sov. J. Sov. Phys. 12, 589 (1971).

105. S. R. Beane and P. F. Bedaque, (2000), hucl-th/0005052.

106. A. C. Phillips and G. Barton, Phys. Lett 6, 378 (1969).

107. I. R. Afnan and F. J. D. Serduke, Phys. Lett. B 44, 143 (1973).

108. P. F. Bedaque, H. W. Hammer, and U. van Kolck, (1999), nuclth/9906032.

109. E. Wigner, Phys. Rev. 51, 106 (1937).

110. T. Mehen, I. W. Stewart, and M. B. Wise, Phys. Rev. Lett. 83, 931 (1999), hep-ph/9902370.

111. K. Wilson, talk presented at the Institute for Nuclear Theory, University of Washington, July 2000. .

112. S. Fleming, T. Mehen, and I. W. Stewart, Nucl. Phys. A677, 313 (2000), nucl-th/9911001.

113. J. Gegelia, nucl-th/9806028.

114. T. D. Cohen and J. M. Hansen, Phys. Lett. B440, 223 (1998), nuclth/9808006.

115. T. D. Cohen and J. M. Hansen, Phys. Rev. C 59, 13 (1999), nuclth/9808038.

116. J. V. Steele and R. J. Furnstahl, Nucl. Phys. A645, 439, nuclth/9808022.

117. D. B. Kaplan and J. V. Steele, Phys. Rev. C60, 064002 (1999), nuclth/9905027.

118. G. Rupak and N. Shoresh, Phys. Rev. C60, 054004 (1999), nuclth/9902077.

119. G. Rupak and N. Shoresh, (1999), nucl-th/9906077.

120. J.-W. Chen, H. W. Griesshammer, M. J. Savage, and R. P. Springer, Nucl. Phys. A644, 245 (1998), nucl-th/9809023.

121. J.-W. Chen, Nucl. Phys. A653, 375 (1999), nucl-th/9810021.

122. J.-W. Chen, H. W. Griesshammer, M. J. Savage, and R. P. Springer, Nucl. Phys. A644, 221 (1998), nucl-th/9806080.

123. D. B. Kaplan, M. J. Savage, R. P. Springer, and M. B. Wise, Phys. Lett. B449, 1 (1999), nucl-th/9807081.

124. M. J. Savage and R. P. Springer, Nucl. Phys. A644, 235 (1998), nuclth/9807014.

125. M. J. Savage and R. P. Springer, (1999), nucl-th/9907069.

126. P. F. Bedaque and H. W. Griesshammer, Nucl. Phys. A671, 357 (2000), nucl-th/9907077.

127. E. Epelbaoum, W. Glockle, A. Kruger, and U.-G. Meissner, Nucl. Phys. A645, 413 (1999), nucl-th/9809084. 
128. M. C. Birse, J. A. McGovern, and K. G. Richardson, Phys. Lett. B464, 169, hep-ph/9807302.

129. C. Ordonez and U. van Kolck, Phys. Lett. B291, 459 (1992).

130. C. Ordonez, L. Ray, and U. van Kolck, Phys. Rev. Lett. 72, 1982 (1994).

131. C. Ordonéz, L. Ray, and U. van Kolck, Phys. Rev. C 53, 2086 (1996), nucl-th/9511380.

132. E. Epelbaoum, W. Glockle, and U.-G. Meissner, Nucl. Phys. A637, 107 (1998), nucl-th/9801064.

133. J. L. Friar and S. A. Coon, Phys. Rev. C 49, 1272 (1994).

134. K. Bruckner and K. M. Watson, Phys. Rev. 92, 1023 (1953).

135. M. Takentani, S. Machida, and S. Ohnuma, Prog. Theor. Phys. 7, 45 (1952).

136. U. van Kolck, PhD thesis, University of Texas, 1993.

137. U. van Kolck, Prog. Part. Nucl. Phys. 43, 409 (1999), nucl-th/9902015.

138. E. Epelbaum, W. Glockle, and U.-G. Meissner, (1999), nucl-th/9910064.

139. P. Buttiker and U.-G. Meissner, Nucl. Phys. A668, 97 (2000), hepph/9908247.

140. N. Fettes and U.-G. Meissner, Nucl. Phys. A676, 311 (2000), hep$\mathrm{ph} / 0002162$.

141. M. Sugawara and S. Okubo, Phys. Rev. 117, 611 (1960).

142. V. G. J. Stoks and T. A. Rijken, Nucl. Phys. A613, 311 (1997).

143. G. E. Brown and J. W. Durso, Phys. Lett. B35, 120 (1971).

144. W. N. Cottingham, M. Lacombe, B. Loiseau, J. M. Richard, and R. V. Mau, Phys. Rev. D8, 800 (1973).

145. M. Lacombe et al., Phys. Rev. C 21, 861 (1981).

146. M. R. Robilotta and C. A. da Rocha, Nucl. Phys. A615, 391 (1997), nucl-th/9611056.

147. K. A. Scaldeferri, D. R. Phillips, C.-W. Kao, and T. D. Cohen, Phys. Rev. C 56, 679 (1997), nucl-th/9610049.

148. R. Machleidt, K. Holinde, and C. Elster, Phys. Rep. 149, 1 (1987).

149. C. H. Hyun, D. P. Min, and T. S. Park, Phys. Lett. B473, 6 (2000), nucl-th/9908073.

150. J. C. Pupin and M. R. Robilotta, Phys. Rev. C60, 014003 (1999), nucl-th/9903028.

151. N. Kaiser, Phys. Rev. C62, 024001 (2000), nucl-th/9912054.

152. M. C. M. Rentmeester, R. G. E. Timmermans, J. L. Friar, and J. J. de Swart, Phys. Rev. Lett. 82, 4992 (1999), nucl-th/9901054.

153. Nuclear Physics with Effective Field Theory, ed. R. Seki, U. van Kolck, and M. J. Savage, (World Scientific, Singapore, 1998). ISBN 981-023596-8; Nuclear Physics with Effective Field Theory II, ed. P. F. Be- 
daque, M. J. Savage, R. Seki, and U. van Kolck, (World Scientific, Singapore, 1999). ISBN 981-02-4181-X.

154. S. R. Beane, C. Y. Lee, and U. van Kolck, Phys. Rev. C 52, 2914 (1995), nucl-th/9506017.

155. S. R. Beane, V. Bernard, T.-S. H. Lee, U.-G. Meissner, and U. van Kolck, Nucl. Phys. A618, 381 (1997), hep-ph/9702226.

156. V. Bernard, H. Krebs, and U.-G. Meissner, Phys. Rev. C61, 058201 (2000), nucl-th/9912033.

157. S. R. Beane, V. Bernard, T. S. H. Lee, and U. G. Meissner, Phys. Rev. C57, 424 (1998), nucl-th/9708035.

158. S. R. Beane, M. Malheiro, D. R. Phillips, and U. van Kolck, Nucl. Phys. A656, 367, nucl-th/9905023.

159. T.-S. Park, K. Kubodera, D.-P. Min, and M. Rho, Nucl. Phys. A646, 83 (1999), nucl-th/9807054.

160. V. Bernard, N. Kaiser, J. Kambor, and U. G. Meissner, Nucl. Phys. B388, 315 (1992).

161. V. Bernard, H. W. Fearing, T. R. Hemmert, and U. G. Meissner, Nucl. Phys. A635, 121 (1998), hep-ph/9801297.

162. B. Kubis and U.-G. Meissner, (2000), hep-ph/0007056.

163. J. L. Friar, Ann. Phys. (N.Y.) 81, 332 (1973).

164. T.-S. Park, K. Kubodera, D.-P. Min, and M. Rho, Phys. Lett. B472, 232 (2000), nucl-th/9906005.

165. D. O. Riska, Prog. Part. Nucl. Phys. 11, 199 (1984).

166. R. Schiavilla and D. O. Riska, Phys. Rev. C43, 437 (1991).

167. D. R. Phillips and T. D. Cohen, Nucl. Phys. A668, 45 (2000), nuclth/9906091.

168. F. Schmidt-Kaler, D. Leibfried, M. Weitz, and T. W. Hansch, Phys. Rev. Lett. 70, 2261 (1993).

169. J. Martorell, D. W. L. Sprung, and D. C. Zheng, Phys. Rev. C 51, 1127 (1995).

170. M. E. Schulze et al., Phys. Rev. Lett. 52, 597 (1984).

171. V. F. Dmitriev et al., Phys. Lett. B. 157, 143 (1985).

172. R. Gilman et al., Phys. Rev. Lett. 65, 1733 (1990).

173. M. Garcon et al., Phys. Rev. C49, 2516 (1994).

174. M. Ferro-Luzzi et al., Phys. Rev. Lett. 77, 2630 (1996).

175. M. Bouwhuis et al., Phys. Rev. Lett. 82, 3755 (1999), nucl-ex/9810004.

176. V. Bernard, N. Kaiser, and U.-G. Meissner, Z. Phys. C70, 483 (1996), hep-ph/9411287.

177. J. H. Koch and R. M. Woloshyn, Phys. Rev. C16, 1968 (1977).

178. J. M. Laget, Phys. Rept. 69, 1 (1981). 
179. R. V. Reid, Ann. Phys. 50, 411 (1968).

180. M. M. Nagels, T. A. Rijken, and J. J. de Swart, Phys. Rev. D 17, 768 (1978).

181. R. de Tourrell and D. Sprung, Nucl. Phys. A210, 193 (1973).

182. J. C. B. et al, Phys. Rev. C57, 3203 (1998).

183. V. Bernard, N. Kaiser, and U.-G. Meissner, Nucl. Phys. A607, 379 (1996), hep-ph/9601267.

184. H. B. van den Brink et al., Nucl. Phys. A612, 391 (1997).

185. M. O. Distler et al., Phys. Rev. Lett. 80, 2294 (1998).

186. V. Bernard, N. Kaiser, and U. G. Meissner, Phys. Lett. B309, 421 (1993), hep-ph/9304275.

187. D. Chatellard et al., Phys. Rev. Lett. 74, 4157 (1995).

188. D. Sigg et al., Phys. Rev. Lett. 75, 3245 (1995).

189. D. Sigg et al., Nucl. Phys. A609, 269 (1996).

190. R. Koch, Nucl. Phys. A448, 707 (1986).

191. R. A. Arndt, I. I. Strakovsky, R. L. Workman, and M. M. Pavan, Phys. Rev. C52, 2120 (1995), nucl-th/9505040.

192. V. Bernard, N. Kaiser, and U.-G. Meissner, Nucl. Phys. A615, 483 (1997), hep-ph/9611253.

193. M. Mojzis, Eur. Phys. J. C2, 181 (1998), hep-ph/9704415.

194. V. Bernard, N. Kaiser, and U. G. Meissner, Nucl. Phys. B383, 442 (1992).

195. V. Bernard, N. Kaiser, J. Kambor, and U. G. Meissner, Nucl. Phys. B388, 315 (1992).

196. J. Tonnison, A. M. Sandorfi, S. Hoblit, and A. M. Nathan, Phys. Rev. Lett. 80, 4382 (1998), nucl-th/9801008.

197. D. L. Hornidge et al., Phys. Rev. Lett. 84, 2334 (2000), nucl-ex/9909015.

198. T. Wilbois, P. Wilhelm, and H. Arenhovel, Few Bod. Sys. 9, 263 (1995).

199. M. I. Levchuk and A. I. L'vov, (1998), nucl-th/9809034.

200. J. J. Karakowski and G. A. Miller, Phys. Rev. C60, 014001 (1999), nucl-th/9901018.

201. D. L. Hornidge et al., Phys. Rev. Lett. 84, 2334 (2000), nucl-ex/9909015.

202. E. Jenkins and A. V. Manohar, Phys. Lett. B255, 558 (1991).

203. M. Chemtob and M. Rho, Nucl. Phys. A163, 1 (1971).

204. K. Kubodera, J. Delorme, and M. Rho, Phys. Rev. Lett. 40, 755 (1978).

205. M. Rho, Phys. Rev. Lett. 66, 1275 (1991).

206. M. Kamionkowski and J. N. Bahcall, Astrophys. J. 420, 884 (1994), astro-ph/9305020.

207. C. da Rocha, G. Miller, and U. van Kolck, Phys. Rev. C61, 034613 (2000), nucl-th/9904031. 
208. B. Y. Park, F. Myrher, J. R. Morones, and T. Meissner, Phys. Rev. C 53, 1519 (1996), nucl-th/9512023.

209. M. J. Savage, Phys. Rev. C55, 2185 (1997), nucl-th/9611022.

210. C. Hanhart, J. Haidenbauer, M. Hoffmann, U.-G. Meissner, and J. Speth, Phys. Lett. B424, 8 (1998), nucl-th/9707029.

211. V. Dmitrasinovic, K. Kubodera, F. Myhrer, and T. Sato, Phys. Lett. B465, 43 (1999), nucl-th/9902048.

212. E. Gedalin, A. Moalem, and L. Razdolskaya, Phys. Rev. C60, 031001 (1999).

213. S. Ando, T.-S. Park, and D.-P. Min, nucl-th/0003004.

214. U. van Kolck, G. A. Miller, and D. O. Riska, Phys. Lett. B388, 679 (1996), nucl-th/9607026.

215. V. Bernard, N. Kaiser, and U.-G. Meissner, Eur. Phys. J. A4, 259 (1999), nucl-th/9806013.

216. C. Hanhart, U. van Kolck, and G. A. Miller, nucl-th/0004033.

217. D. Huber, J. L. Friar, A. Nogga, H. Witala, and U. van Kolck, nuclth/9910034.

218. U. van Kolck, Phys. Rev. C 49, 2932 (1994).

219. C. Hanhart, U. van Kolck, and G. A. Miller, Phys. Rev. C 49, 2932 (1994).

220. D. Huber et al., Few-Body Systems 0, 1 (2000), nucl-th/9910034.

221. E. Epelbaum et al., (2000), nucl-th/0007057.

222. R. Machleidt, Advances in Nuclear Physics 19, 189 (1989).

223. R. B. Wiringa, R. A. Smith, and T. L. Ainsworth, Phys. Rev. C 29, 1207 (1984).

224. R. Machleidt, F. Sammarucca, and Y. Song, Phys. Rev. C 53, 1483 (1996).

225. W. C. Haxton and C. L. Song, (1999), nucl-th/9906082.

226. W. C. Haxton and C. L. Song, Phys. Rev. Lett. 84 (2000), nuclth/9907097.

227. M. Lutz, Nucl. Phys. A670, 214 (2000).

228. R. J. Furnstahl and B. D. Serot, Nucl. Phys. A663, 513 (2000), nuclth/9907073.

229. H. W. Hammer and R. J. Furnstahl, (2000), nucl-th/0004043.

230. B. Krippa, (2000), hep-ph/0006305.

231. E. Caurier et al., Phys. Rev. C59, 2033 (1999), nucl-th/9809068.

232. P. Navratil and B. R. Barrett, Phys. Rev. C59, 1906 (1999), nuclth/9812062.

233. S. E. Koonin, D. J. Dean, and K. Langanke, Phys. Rept. 278, 1 (1997), nucl-th/9602006. 
234. M. Honma, T. Mizusaki, and T. Otsuka, (1996), nucl-th/9609043.

235. D. C. Zheng, B. R. Barrett, J. P. Vary, W. C. Haxton, and C. L. Song, Phys. Rev. C52, 2488 (1995).

236. C. Bloch and J. Horowitz, Nucl. Phys. 8, 91 (1958).

237. B. R. Barrett and M. W. Kirson, Ad. in Nucl. Phys. 6, 219 (1973).

238. T. T. S. Kuo and E. Osnes, Lecture Notes in Physics 364 (1990).

239. T. H. Shucan and H. A. Weidenmuller, Ann. Phys. (NY) 73, 108 (1972).

240. T. H. Shucan and H. A. Weidenmuller, Ann. Phys. (NY) 76, 483 (1973).

241. R. Haydock, J. Phys. A 7, 2120 (1974).

242. M. Moshinsky, (Gordon and Breach, New York, 1969).

243. C.-L. Song, (Univ. Washington Ph.D, Seattle, 1998).

244. B. R. Barrett, Czech. J. of Phys. 49, 1 (1999).

245. W. C. Haxton and T. Luu, submitted to Phys. Rev. Lett. (2000).

246. T. A. Brody and M. Moshinsky, (Gordon and Breach, New York, 1967). 